alpine space - man \& environment vol. 6

Roland Schmidt, Christoph Matulla, Roland Psenner (Hrsg.)

\title{
Klimawandel in Österreich \\ Die letzten 20.000 Jahre ... und ein Blick voraus
}





\section{SERIES}

alpine space - man \& environment: vol. 6

Series Editors: Roland Psenner, Reinhard Lackner, Axel Borsdorf

Band 6

iup • innsbruck university press

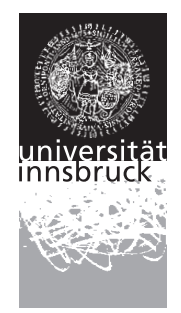


(C) innsbruck university press, 2009

Universität Innsbruck, Vizerektorat für Forschung

1. Auflage

Alle Rechte vorbehalten.

Cover: Gregor Sailer

Coverfoto: @ Büro für Öffentlichkeitsarbeit, Universität Innsbruck (0.i.F.)

Layout: Reinhard Lackner

Produktion: Fred Steiner, Rinn

www.uibk.ac.at/iup

www.uibk.ac.at/alpinerraum/publications/

ISBN 978-3-902571-89-2 
Roland Schmidt, Christoph Matulla, Roland Psenner (Hrsg.)

\section{Klimawandel in Österreich}

Die letzten 20.000 Jahre ... und ein Blick voraus 



\section{Inhalt}

Vorwort

Roland Schmidt

Gletscher und Klima im Alpinen Spätglazial und frühen Holozän . . . . . . . . . . . 5

Hanns Kerschner

Die Bedeutung des Längsees in Kärnten für die Rekonstruktion der Klima- und

Seenentwicklung am Ende der letzten Eiszeitt . . . . . . . . . . . . . . . . 27

Schmidt, R., Drescher-Schneider, R., Huber, K. und Weckstöm, K.

Alpine Dendrochronologie - Untersuchungen zur Kenntnis der holozänen

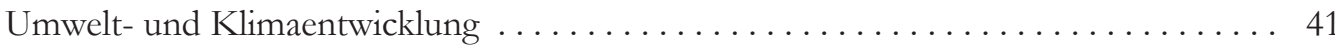

Kurt Nicolussi

Klimaschwankungen und -trends des älteren Holozäns in den südlichen

Niederen Tauern: multidisziplinäre Auswertung eines Sedimentkerns

aus dem Oberen Landschitzsee (Lungau) . . . . . . . . . . . . . . . . . . . . . 55

Schmidt, R., Kamenik, C., Kaiblinger, C. und Tessadri, R.

Wie alt sind Blockgletscher in den Österreichischen Alpen?

Das Beispiel der Blockgletscher im Dösener Tal, Ankogelgruppe, datiert mit Hilfe der Schmidt-Hammer Methode . . . . . . . . . . . . . . . . . . . . . . . 65

Andreas Kellerer-Pirklbaner

Prähistorische Besiedlung von zentralen Alpentälern in Bezug zur Klimaentwicklung. . . . . . . . . . . . . . . . . . . . . . . . . . . 77

Oeggl, K. und Nicolussi, K.

Der Einfluss des Klimas auf die Hochlagennutzung in den südlichen

Niederen Tauern (Lungau) während der letzten 4000 Jahre . . . . . . . . . . . . 87

Schmidt, R, Kamenik, C. und Roth, M.

Hallstatts bronzezeitliche Almen . . . . . . . . . . . . . . . . . . . . . . 97

Franz. Mandl

Die Schwarzkiefer als Niederschlagsindikator für Ostösterreich . . . . . . . . . . 105

Grabner, M. und Holawe, F.

Die Sedimentablagerungen des Mondsees (Oberösterreich) als ein Archiv

extremer Abflussereignisse der letzten 100 Jahre . . . . . . . . . . . . . . . . . 115

Swierczynski, T., Lauterbach, S., Brauer, A. und Dulski P. 
Paläoklimainformationen aus Kenngrößen der Gletschermassenbilanz -

Beispiele für die Alpen seit der ausgehenden Kleinen Eiszeit . . . . . . . . . . . . . 127

Wolfgang Schöner

Klimarekonstruktion der instrumentellen Periode - Probleme und

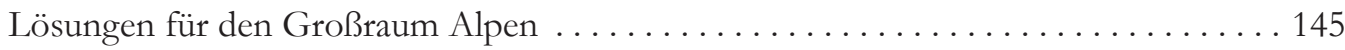

Reinhard Böhm

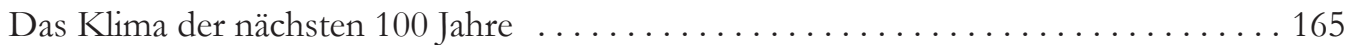

Christoph Matulla

Zusammenfassung und Ausblick . . . . . . . . . . . . . . . . . . . 181

Schmidt, R., Matulla, C. und Psenner, R. 


\title{
Klima im Wandel
}

\subsection{Jahre Klimaentwicklung in Österreich (Proxis, Daten, Szenarien)}

\section{Climate Change \\ 20,000 years of climate development in Austria (proxies, data, scenarios)}

\author{
Roland Schmidt \\ Institut für Limnologie, Österreichische Akademie der Wissenschaften, Mondsee, Österreich
}

Wenn schon kurzfristige Wetterprognosen schwierig sind, wie schwierig wird erst eine Prognose des Klimas sein? - eine oft gestellte Frage mit ambivalenten Antworten. Was ist nun Klima? Klima ist - kurz gesagt - Wetter mal Zeit und in der Regel betrachtet man einen Zeitraum von 30 Jahren, d.h. die meisten Vergleiche beziehen sich auf den Mittelwert von 1961 bis 1990. Selbst die Messdaten der letzten 250 Jahre in den Alpen reichen aber nicht aus, um die komplexen Klimaabläufe zur Gänze zu verstehen. Wenn wir mit indirekten, sogenannten Proxy-Daten, die Zeitserien verlängern (back to the future), kann das unsere Probleme lösen? Seit der letzten Eiszeit sind etwas mehr als 20.000 Jahre vergangen, ein Abschnitt, von dem wir nicht wissen, ob er vielleicht nur Teil eines Interglazials ist, wenn wir auf den Wechsel von Kalt- (Glaziale) und Warmzeiten (Interglaziale) der vorangegangenen zumindest vier großen Vereisungen in den Alpen zurückblicken, die das Eiszeitalter (Pleistozän) kennzeichnen. Allgemein lassen die Übergangszeiten von diesen Kaltzu Warmzeiten und umgekehrt eine größere Instabilität und höhere Schwankungsbreite des Klimas erkennen als die Interglaziale selbst. Dennoch zeigen auch die Warmzeiten Oszillationen mit zum Teil zyklischer Abfolge und kurzfristige, zumeist azyklische, Fluktuationen, die geologische, mineralogische, physikalische, chemische und biologische Spuren hinterlassen. Neben den Gletschern gibt es eine Reihe weiterer klimaempfindlicher Indikatoren (= Proxis), wie zum Beispiel stabile Isotope im Eis und in kalkschaligen Organismen und Höhlensedimenten, Organismen in Seeablagerungen, die Wald- und Baumgrenze, Hölzer und Baumringe, Böden usw., die uns über die Variabilität des Klimas vor Eingriff des Menschen Auskunft geben. Je besser wir solche Signale unterschiedlicher Klimazeiger zu einem Gesamtbild 
vereinen, desto sicherer wird unsere Aussage über die Klimadynamik sein und umso besser werden wir ihre Mechanismen verstehen. In die letzten ca. 10.000 Jahre fällt die Entwicklung und Diversität der menschlichen Kulturen. War der Mensch vor der Neuzeit weitgehend vom Klima abhängig, so greift er seit der industriellen Revolution im 19. Jahrhundert selbst aktiv in das Klimageschehen ein. Es gilt als sicher, dass die Menschheit Anteil an der heutigen Klimaerwärmung hat und sich diese offensichtlich beschleunigt. Die Zeichen dieses "global warming" sind u. a. durch das Abschmelzen der Alpengletscher während der letzten 10 Jahre auch für den Laien deutlich sichtbar. Die Zukunftsszenarien, die uns für die nächsten 20 Jahre eine globale Temperaturzunahme um $0,4^{\circ} \mathrm{C}$ (IPCC Klimabericht 2007) und für die nächsten 100 Jahre für Europa eine solche von mehr als $3{ }^{\circ} \mathrm{C}$ voraussagen, sind auf komplexe Simulationen (Abschätzungen, Projektionen) aufgebaut, die im wesentlichen mittels Emissionsszenarien von Treibhausgasen getrieben werden. Je

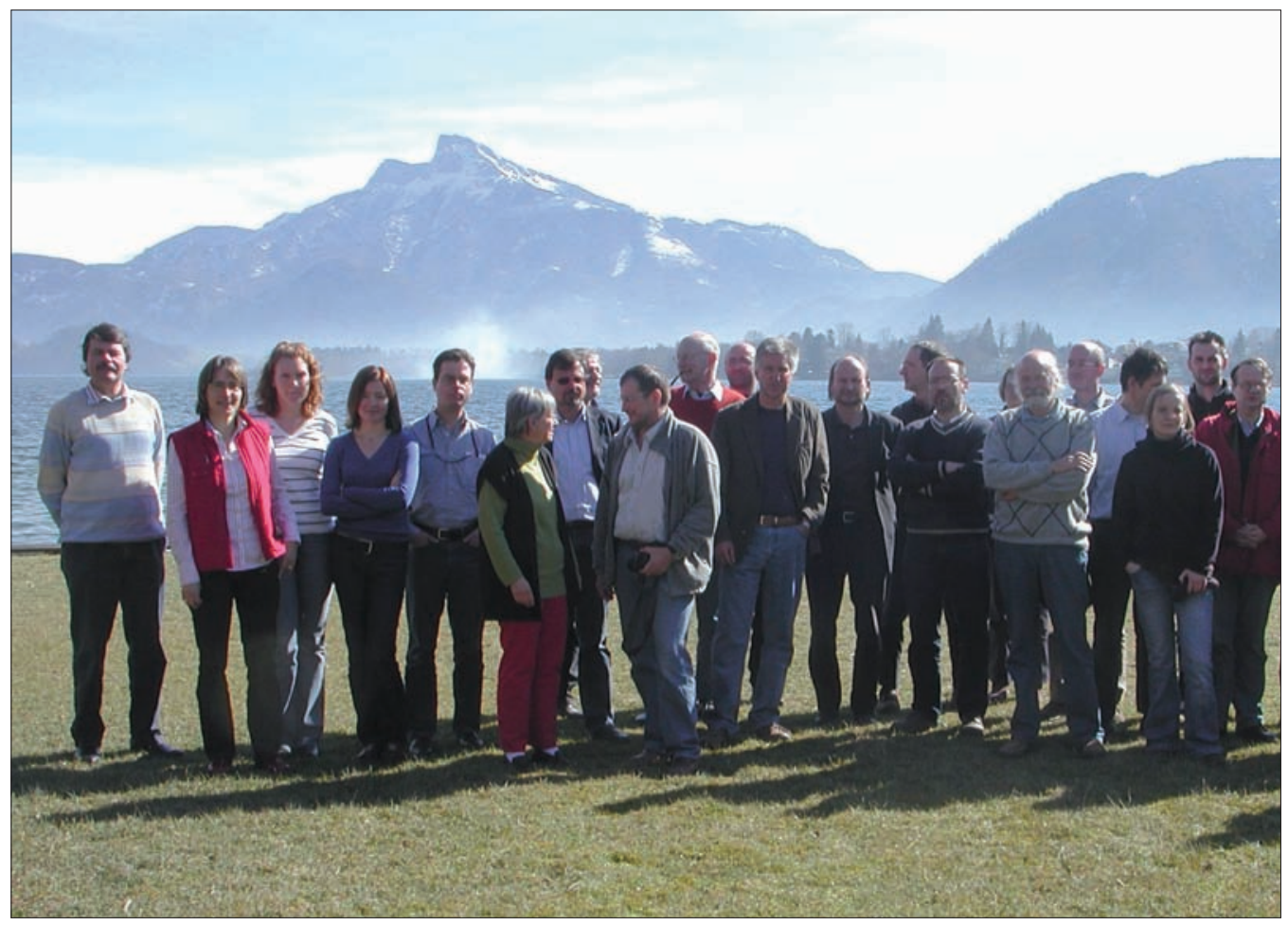

Abb. 1: TeilnehmerInnen am PALDAT-Meeting 2008 am Institut für Limnologie der Österreichischen Akademie der Wissenschaften, Mondsee

Fig. 1: Participants of the PALDAT-Meeting 2008, held at the Institute for Limnology of the Austrian Academy of Sciences, Mondsee 
weiter wir jedoch in die Zukunft blicken, desto größer wird die Unsicherheit der Vorhersage, wenn wir nur beispielsweise an den möglichen Einfluss der natürlichen Klimavariabilität oder an Rückkoppelungseffekte denken, wie zum Beispiel die regional stark unterschiedliche Entwicklung der Niederschlagsverteilung.

Die Ergebnisse der Klimaforschung in Österreich sind zumeist in den Fachzeitschriften der einzelnen Forschungsbereiche verstreut. Daher haben wir eine Initiative in Form eines Workshops mit dem Namen PALDAT (Vernetzung von Klimasignalen unterschiedlicher Paläo-Indikatoren, Zeitskalen, der instrumentellen Periode und der Szenarienforschung) gestartet, der am Institut für Limnologie der Österreichischen Akademie der Wissenschaften in Mondsee vom 10. bis 11. März 2008 abgehalten wurde (Abb. 1), mit dem Ziel, die einzelnen Disziplinen zusammenzuführen und die Ergebnisse aus Österreich einem breiten Publikum zugänglich zu machen. Ausgewählte Beiträge aus den einzelnen Disziplinen, ohne Anspruch auf Vollständigkeit, werden nun in diesem Band veröffentlicht. Sie sollen einen Einblick in das Klimageschehen in Österreich während der letzten 20.000 Jahre und dessen Auswirkungen vermitteln, einen Brückenschlag in die Zeit instrumenteller Klimadaten der letzten Jahrhunderte machen, sowie einen Blick in die Zukunft wagen. Die in den Beiträgen angeführten Zitate sollen den Interessierten das Tor zur Spezialliteratur öffnen. Allgemein soll der Band das Interesse und die Neugier an der Klimaforschung wecken und Denkanstöße für den kritischen Zugang zum heutigen Klima im Wandel ("global change") vermitteln. 


\title{
Gletscher und Klima im Alpinen Spätglazial und frühen Holozän
}

\author{
Hanns Kerschner
}

Institut für Geographie der Universität Innsbruck, Innrain 52, 6020 Innsbruck

\section{Abstract}

Glaciers and climate during the Alpine Lateglacial and early Holocene

\begin{abstract}
${ }^{10} \mathrm{Be}$ dating of prominent glacier advances (stadials) in the Alps supports the correlation of the Gschnitz stadial (>16 ka) with the Heinrich 1 ice rafting event, the Egesen-maximum advance ( 12.3 - $12.4 \mathrm{ka})$ with the early Younger Dryas and the Kartell advance $(\sim 10.8 \mathrm{ka})$ with a climatic fluctuation during the early Preboreal. The age of the Kromer advance $(\sim 8.4$ ka) remains somewhat enigmatic. The interpretation of the glacial record with glacierclimate models (summer temperature change $(\Delta \mathrm{Ts})$, precipitation change $(\Delta \mathrm{P})$ ) shows that during the Gschnitz stadial summers were $\sim 10^{\circ} \mathrm{C}$ colder than at present and annual precipitation was reduced to $\sim 25-50 \%$ of modern annual sums. During the Egesen maximum, $\Delta \mathrm{P}$ depends on the estimated $\Delta \mathrm{Ts}$. For a $\Delta \mathrm{Ts}$ of $-3.5^{\circ} \mathrm{C}$, precipitation along the northern fringe of the Eastern Alps was about similar to modern sums, in the sheltered central valleys $\Delta \mathrm{P}$ was in the order of -20 to $-30 \%$. A scenario with a $\Delta \mathrm{Ts}$ of $-5^{\circ} \mathrm{C}$ leads to generally drier conditions $(\sim-15 \%$ vs. $\sim-50 \% \Delta \mathrm{P})$ but the spatial pattern of precipitation change remains the same. During the Kartell advance, it was only slightly cooler than during the Little Ice Age, while precipitation was rather similar.
\end{abstract}

\section{Einleitung}

Während des "Alpinen Spätglazials" schmolzen die großen eiszeitlichen Gletscher vom Maximum im Alpenvorland bis auf jene Größe zurück, die sie während des Hochstandes von 1850 erreichten. Dieser Rückzug lief nicht gleichmäßig ab, sondern es wechselten Zeitabschnitte eines raschen Zurückschmelzens, des Stagnierens und erneuter, immer kleinerer Gletschervorstöße, einander ab. Die Ursachen dieser Gletscherschwankungen liegen in den Veränderungen des weltweiten Klimas, das im europäisch-atlantischen Sektor damals noch ganz anders als heute war. Die Existenz der großen Eisschilde in Nordamerika und Skandinavien, wesentliche Unterschiede in der Meerestemperatur, in der Salinität und in den Zirkulationsverhältnissen im Atlantik und eine zumindest jahreszeitlich wesentlich andere Meereisbedeckung als heute waren seine Randbedingungen. 
Diese Gletschervorstöße haben in den Tälern und Karen an vielen Stellen auffällige Moränensysteme hinterlassen. Dazu kommen große Blockgletschersysteme, die die frühere Verbreitung von diskontinuierlichem Permafrost nachzeichnen. Alle diese Ablagerungen und Formen können kartiert und klimageschichtlich interpretiert werden. In jüngster Zeit eröffnet "Google Earth" die Möglichkeit, viele Gebiete in einzigartiger Qualität dreidimensional betrachten zu können. Darauf wird im Text jeweils hingewiesen, für die typischen Stellen werden die geographischen Koordinaten eines Punktes angegeben, mit dessen Hilfe sie sich auffinden lassen.

\section{Warum Gletscher?}

Für klimageschichtliche Studien haben Gletscher mehrere Vorteile, aber auch einige Grenzen. Sie sind über ihren Massenhaushalt direkt mit ihrer klimatischen Umwelt verbunden. Deshalb kann man bestimmte Größen, die von früheren Gletscherausdehnungen abgeleitet werden, unmittelbar physikalisch-klimatisch interpretieren. In erster Linie handelt es sich um die Höhe ihrer Gleichgewichtslinie und um deren Schwankungen ("Schneegrenzdepression"). An der Gleichgewichtslinie eines Gletschers ist die Akkumulation eines Jahres, die vor allem durch den Niederschlag gesteuert wird, genau gleich der Ablation, die durch die Wärmeströme zur Gletscheroberfläche hervorgerufen wird (vgl. Hoinkes 1970, Gross et al. 1977).

Gletscher sind physikalisch relativ "simple Wesen", deshalb ist der Spielraum bei der klimageschichtlichen Interpretation verhältnismäßig klein; die glaziologischen Befunde lassen sich einfach in herkömmliche Klimadaten (mittlere Sommertemperatur, Jahresniederschlag) übersetzen. Wenn Gletschervorstöße hinreichend genau datiert sind, kann man sie mit kontinuierlichen Befunden, wie z.B. datierten Pollenoder Sauerstoffisotopenprofilen verbinden. Biologische Proxydaten, also Befunde, die eine Klimainformation enthalten, kann man in derartigen Fällen dazu verwenden, um die Gletscherdaten weiter zu interpretieren.

Normalerweise gibt es nur Informationen über Gletschervorstöße, da nur dann Moränenwälle abgelagert wurden, die man heute kartieren kann. Über die Zeit dazwischen weiß man aus der Gletschergeschichte nichts, und diese Zeiträume können eine beträchtliche Länge aufweisen. Damit eignen sich Gletscher für eine verhältnismäßig exakte klimatische Interpretation von verhältnismäßig kurzen Zeitabschnitten, meist in der Größenordnung von einigen Jahrzehnten bis einigen Jahrhunderten. Ähnlich wie von vielen biologischen Proxies bekommt man von Gletschern eine „sommer- 
lastige" Temperaturinformation; winterliche Temperaturverhältnisse lassen sich kaum und dann nur sehr indirekt und qualitativ erschließen.

\section{Die regionale Stratigraphie}

Bereits vor einem Jahrhundert war klar, daß der Eisrückzug von den hocheiszeitlichen Maxima im Alpenvorland nicht kontinuierlich ablief, sondern von einer ganzen Reihe erneuter Gletschervorstöße unterbrochen wurde. Penck und Brückner (1901/09) gliederten diese Vorstoßphasen in die Stadiale "Bühl", "Gschnitz" und "Daun", wobei das Gebiet des eiszeitlichen Inngletschers den Bezugsrahmen darstellte. Für die Zuweisung eines Gletscherstandes zu einem Stadial war die Schneegrenzdepression (Differenz zwischen der Gleichgewichtslinie des Gletscherstandes und der "heutigen" Gleichgewichtslinie) am bedeutendsten.

Im Laufe der Jahre wurde dieses Schema mehrfach erweitert, umgedeutet oder durch gänzlich andere Gliederungen ersetzt (Abb. 1). So wurde im Laufe der Zeit gleiches ungleich und ungleiches gleich benannt. Auch die bedeutenden Grenzen zog
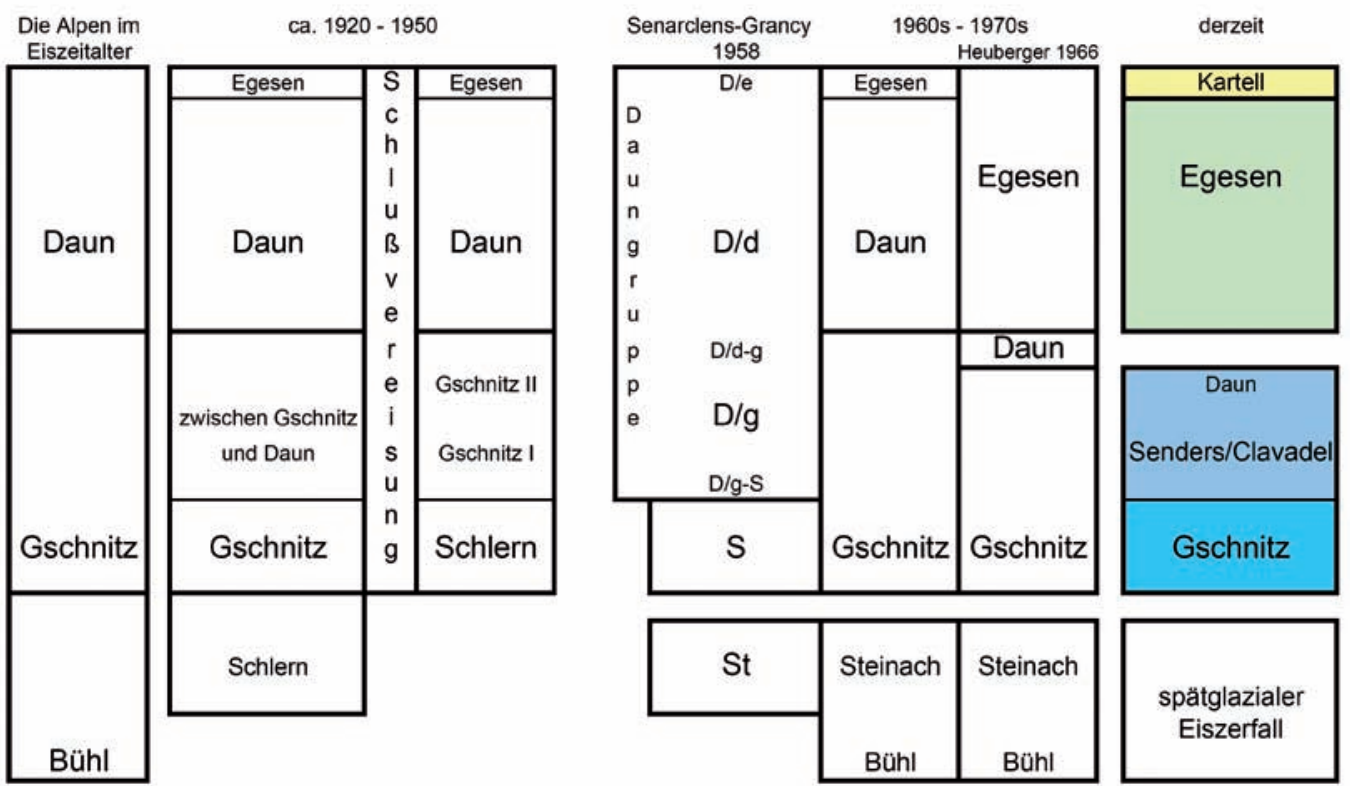

Abb. 1: Textkritischer Überblick der Entwicklung der spätglazialen Moränenstratigraphie in den Ostalpen seit den „Alpen im Eiszeitalter" (zur Literatur siehe Kerschner 1986).

Fig. 1. Critical overview of the development of late glacial moraine stratigraphy in the Eastern Alps since „Alpen im Eiszeitalter“ (see Kerschner 1986 for references). 
man an unterschiedlichen Stellen der Moränenabfolgen. Eine genauere Betrachtung der Abbildung 1 zeigt, wie sich die Bedeutung der einzelnen stratigraphischen Begriffe im Laufe der Zeit verschoben hat. Es fehlte die Möglichkeit zur absoluten Datierung der Moränen und die Hilfsmittel bei der Kartierung, wie z.B. topographische Karten, waren oft sehr schlecht. So sind die verschiedenen Kataloge an Kriterien und Beurteilungsschemata, die für die stratigraphische Zuweisung eines Gletscherstandes verwendet wurden und werden, nichts anderes als ein Ersatz für die fehlenden absoluten Alter.

Ein derartiges Schema wird auch heute noch für die Geländearbeit verwendet. Es baut auf einer Erweiterung der ursprünglichen Stadialgliederung der "Alpen im Eiszeitalter" auf und geht in seinen wesentlichen Zügen auf die Arbeit von Heuberger (1966) in den nördlichen Stubaier Alpen zurück (Abb. 1). Entlang einer Längsachse der Zentralalpen zwischen den Hohen Tauern und der Mont Blanc-Gruppe und anscheinend auch in weiten Teilen der Südalpen bewährt es sich sehr gut. Dieses Gliederungsschema verwendet einen Katalog von Kriterien, die im Gelände und anhand von Karten gewonnen werden. Er umfaßt die Lage der Moränen zueinander, die Mikromorphologie der Moränen (u.a. Formfrische, Überprägung durch Solifluktion, Blockigkeit, Erosion, Größe) relativ zum Ausgangsmaterial, die Schneegrenzdepression gegenüber dem Hochstand von 1850, die Höhe der Schneegrenze des betrachteten Gletschers im Vergleich zur Situation in den Nachbartälern, sowie glaziologische Parameter, die eine Aussage über die Aktivität des Gletschers zulassen. Dabei geht man davon aus, daß wesentliche morphologische Grenzen auch wesentliche und sinnvolle Zeitgrenzen sind. Die absoluten Datierungen, die in den letzten Jahren gewonnen wurden, zeigen, daß dieses differentialdiagnostische Gliederungsschema prinzipiell vernünftig ist. In den Nordalpen und am westlichen Rand, also im atlantischen Randsaum der Alpen, hat man damit allerdings Schwierigkeiten. Eine sehr detaillierte regionale Gliederung von Schoeneich (1998) in den waadtländischen Préalpes oder die Abfolge der Gletschervorstöße im Karwendel (Kerschner 1992) läßt sich mit der zentralalpinen Gliederung zur Zeit nur mit viel Mühe und einigen Unsicherheiten verbinden.

\section{Datierungsversuche}

Bis in die Neunzigerjahre waren Radiokarbon-Mindestalter im Zusammenhang mit pollenanalytischen Untersuchungen die einzigen Hinweise auf das absolute Alter der 
Moränensysteme (Patzelt 1972, Patzelt \& Bortenschlager 1973). Dabei zeigte sich, daß die Moränenserie des Egesenstadiums wohl der Kaltphase der Jüngeren Dryas (= Grönland Stadial 1 der $\mathrm{O}^{16 / 18}$ Isotopenstratigraphie Grönländischer Eiskerne, 12.7 - 11.5 ka, Björck et al. 1998, Walker et al. 1999) entspricht, während alle anderen Stadien mindestens älter als das Alleröd - Interstadial, wahrscheinlich aber älter als der Beginn des Bölling - Interstadials (14.7 ka) sind. Bei beiden handelt es sich um pollenanalytisch erfaßte Stratozonen Mitteleuropas des spätglazialen Interstadials (= Grönland Interstadial 1). Im Einklang mit der zunehmenden Möglichkeit, Radiokarbondaten zu kalibrieren, wurden diese Stadiale kalendarisch immer älter. Die Radiokarbondatierung wurde allerdings durch zwei Umstände behindert. Einerseits findet man gerade in der Zeit vor dem Beginn des Bölling kaum hinreichendes organisches Material in Beziehung zu den Moränensystemen und andererseits bestehen gerade während der interessanten Zeitabschnitte, wie etwa im frühen Präboreal vor rund 9.600 Radiokarbonjahren, lange ${ }^{14} \mathrm{C}$ - Plateaus, wo die Radiokohlenstoffdatierung mit großen Unsicherheiten behaftet ist (Becker und Kromer 1986, 1993).

Entscheidende Fortschritte konnten erst durch die Oberflächenexpositionsdatierung von Moränen mit kosmogenen Radionukliden $\left({ }^{10} \mathrm{Be},{ }^{26} \mathrm{Al},{ }^{36} \mathrm{Cl}\right.$; vgl. Gosse, Phillips 2001, Ivy-Ochs, Kober 2008) erzielt werden. Derzeit wird in Kristallingesteinen fast ausschließlich ${ }^{10} \mathrm{Be}$ für die Datierung verwendet. Damit wird der Zeitraum festgestellt, seit dem Gesteinsoberflächen der sekundären kosmischen Strahlung ausgesetzt sind. Diese Methode der Datierung von Moränen wurde in Europa erstmals von Ivy-Ochs et al. (1996) angewandt. Sie ergibt Mindestalter für die morphologische Stabilisierung des Moränenkörpers. Dieser Zeitraum kann knapp nach dem Gletscherrückzug von der Moräne liegen, muß es aber nicht. Erosionsphasen, eine verzögerte Stabilisierung der Moräne, eine spätere Destabilisierung oder eine nachträgliche verstärkte Oberflächenerosion der Blöcke durch Baumwuchs können zu einer scheinbaren Verjüngung der Moräne beitragen (IvyOchs et al. 2007).

Alle angegebenen Alter in dieser Arbeit sind «Kalenderjahre» vor 1950 in Tausenderjahren (ka). Hier werden nur die Mittelwerte bzw. Altersbereiche angegeben, die Originaldaten können der zitierten Literatur entnommen werden. Die Schneegrenzdepressionen beziehen sich auf den Gletscherhochstand von 1850, der ein gutes Maß für die holozäne Maximalausdehnung darstellt (Gross et al. 1977). 


\section{Der spätglaziale Eiszerfall}

Der großflächige Zerfall der hocheiszeitlichen Gletscher, deren Moränen schon vor rund $20 \mathrm{ka}$ stabilisiert wurden (Ivy-Ochs et al. 2004), dauerte möglicherweise nur einige Jahrhunderte, wobei das Kalben in die damals bestehenden Seen in den großen alpinen Tälern eine wesentliche Rolle gespielt haben kann (van Husen, 2000). In den Ostalpen beschränkten sich die Gletschervorstöße dieses Zeitabschnittes auf kleinere Gletscher und hatten teilweise eisdynamische Ursachen (Reitner 2005, 2007), während größere Talgletschersysteme höchstens in den oberen Abschnitten (Ufermoränenbereich) auf Klimaschwankungen reagierten. Im klassischen Sinne gehören die Stadien „Bühl“ und „Steinach“ (Abb. 1) in diese Phase. Nach einem OSL-Datum (optisch stimulierte Lumineszenz) aus pro-glazialen Sedimenten im unteren Brixental bei Hopfgarten ist dieser Zeitraum um etwa 19.000 Jahre zentriert (Klasen et al. 2007). Sein Ende wird im Traungletschergebiet durch das Radiokarbondatum von Rödschitz im inneren Salzkammergut (Mitterndorfer Becken) (18.0-19.1 ka, van Husen 1977) markiert.

In den Haupttälern der Ostalpen sind weitgespannte Systeme von Eisrandablagerungen die Leitformen dieses Zeitabschnittes, die das Niederschmelzen des Eisstromnetzes nachzeichnen. In den Westalpen mit ihren wesentlich höheren Gipfeln und steileren und kürzeren Gletschern könnten die Verhältnisse etwas anders und vielfältiger gewesen sein (Schoeneich 1998, Coutterand, Nicoud 2005).

\section{Gschnitz, und nachfolgende Stadien}

Der erste besser erfaßbare Gletschervorstoß ist durch die Endmoräne von Trins im

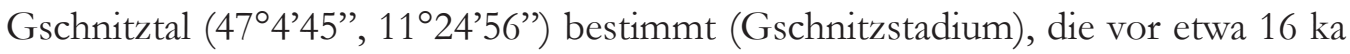
stabilisiert wurde (Ivy-Ochs et al. 2006a). Dieser Zeitraum ist auch durch einige ${ }^{14} \mathrm{C}$ - Mindestalter an vergleichbaren Stellen abgesichert. Die Größe der Moräne, sie ist bis zu $30 \mathrm{~m}$ hoch, legt nahe, daß der Gletscher nach dem Vorstoß mindestens einige Jahrzehnte oder länger mehr oder weniger stationär verharrte. Die Schneegrenze lag im Gschnitztal etwa $700 \mathrm{~m}$ tiefer als um 1850. Die Ursache des Gletschervorstoßes kann in der Klimaverschlechterung gesehen werden, die durch den «Heinrich-1 ice rafting event» im Nordatlantik verursacht wurde; sein Beginn wird um etwa 17.5 bis 17 ka angenommen (Hemming 2004; vgl. auch Schmidt et al., dieser Band). Auf der GRIP - Zeitskala fällt das Gschnitzstadium in das Grönlandstadial 2a. Damit 
würden für den Zeitraum zwischen dem Beginn der Klimaverschlechterung und der Stabilisierung der Moräne rund 1000 - 1500 Jahre zur Verfügung stehen; der Gletschervorstoß des Gschnitzstadiums kann deshalb auch älter als 16.000 Jahre sein. Das Datum von Rödschitz im Salzkammergut und die Wärmeschwankung der Längseeoszillation von ca. 19 - 18 ka, (vgl. Schmidt et al., dieser Band; Schmidt et al. 2002) und damit das Grönlandstadial 2b kann man jedenfalls als Maximalalter sehen.

Das Gschnitzstadium ist bei kleineren und mittelgroßen Gletschern gut kartierbar, dementsprechend ist die Geländeevidenz gerade in den österreichischen Alpen relativ gut. Wenn die Zuordnung der entsprechenden Moränen zu diesem Zeitraum korrekt ist, sollte die Schneegrenzdepression im südlichen Ostalpenraum (Nordseite der Julischen und Karnischen Alpen) etwas stärker gewesen sein als an der Typlokalität (Kerschner, Ivy-Ochs 2008). In jedem Fall kann man davon ausgehen, daß die großen Seitentäler noch vergletschert waren und in günstigen Positionen auch die Haupttäler von Gletscherzungen erreicht wurden. In den höheren Gebieten sollten noch größere zusammenhängende Gletscher bestanden haben. Die Ausdehnung sehr großer Gletschersysteme, wie z.B. des Inngletschers aus dem Engadin, des Rhonegletschers im Wallis oder des Aaregletschers ist jedoch praktisch unbekannt, ebenso die Ausdehnung in den Nordalpen von Tirol westwärts. Die Moränensysteme sind offenbar nicht erhalten oder in den heutigen Talfüllungen verborgen. Auch die Möglichkeit von kalbenden Gletscherenden in inneralpinen Seesystemen (vgl. Poscher 1993) kann man nicht ausschließen.

Durch das relativ hohe Alter der Trinser Moräne bleibt ein Zeitraum von wenigstens 1000 Jahren bis zum Beginn des spätglazialen Interstadials, in dem das Clavadel/ Senders-Stadial und das Daunstadial stattgefunden haben muß. Neue Daten (Maisch in Ivy-Ochs et al. 2008, Studer 2005) zeigen, daß große Teile des Oberengadins bereits vor ca. $15.5 \mathrm{ka}$ eisfrei waren und geben damit auch ein Mindestalter für das Clavadelstadial. Moränen des Clavadel/Sendersstadials sind im Ostalpenraum ziemlich verbreitet anzutreffen. Die Gletscher waren bei einer Schneegrenzdepression in der Größenordnung von 400 bis $500 \mathrm{~m}$ deutlich kleiner als während des Gschnitzstadiums. Die Abtrennung des Daunstadiums (im Sinne von Heuberger 1966) vom Clavadel- bzw. Sendersstadium ist in vielen Fällen nicht einfach. An einigen Stellen gewinnt man den Eindruck, daß es sich dabei eher um kurze Vorstoßperioden im Zuge des allgemeinen Abschmelzens handelt (vgl. Heuberger 1966: 105). Zeitlich wird man auch das Daunstadium noch vor dem Beginn des spätglazialen Interstadials einordnen können (Maisch 1981, 1982). 
Die rasche Erwärmung am Beginn des Bölling beendete endgültig jene Serie von Gletschervorstößen, die mit dem Gschnitzstadium begann. Während des spätglazialen Interstadials kam es zwar zu einer Reihe kleinerer bzw. kurzfristiger Klimaschwankungen, wofür jedoch keine Moränensysteme bekannt sind. Spuren von Gletschervorstößen während dieses Zeitabschnittes von ca. 2000 Jahren wurden offenbar von den nachfolgenden Gletschervorstößen der Jüngeren Dryas ausgelöscht (Ohlendorf 1998).

\section{Egesenstadium}

Heute versteht man unter dem Begriff «Egesenstadium» eine Abfolge von Gletschervorstößen, die eine vielgliedrige Moränenserie hinterließ. Der Maximalvorstoß fällt in den ersten Abschnitt der Jüngeren Dryas. Talwärts von den holozänen Moränen sind die Egesenmoränen die auffälligsten glazialen Ablagerungen und können in allen Gebieten der Alpen gefunden werden, die dafür hoch genug sind (vgl. Literaturverzeichnisse in Ivy-Ochs et al. 2008 und Kerschner et al. 2000; zuletzt Federici et al. 2008). Nach dem Geländebefund lassen sich praktisch immer zwei, in vielen Fällen auch drei Moränengruppen zusammenfassen. Die zahlreichen, wohlausgebildeten Moränensysteme dieses Zeitabschnitts spiegeln instabile klimatische Bedingungen wider, wodurch Rückzugs- und Vorstoßphasen ständig miteinander abwechselten.

Von den älteren Moränensystemen unterscheiden sich jene des Egesenstadiums durch ihre relative Frische und ihre gute Erhaltung. Die Außengrenze des Maximalvorstoßes ist meist auch eine deutliche morphologische Grenze. Ältere Moränen weiter talaus sind meist schlechter erhalten und durch Periglazialprozesse stärker überarbeitet. Diese deutliche geomorphologische Grenze wird bei der Kartierung benutzt, um die Außengrenze des Egesenstadiums festzulegen. Besonders die Moränen der zweiten Phase (Bocktentälli-Phase, Maisch 1981) sind dort, wo es die Umgebung der Gletscher zuläßt, ausgesprochen blockreich. In manchen Gebieten sind die Egesenmoränen regelrechte Landmarken und tragen lokale Flurnamen (Abb. 2). Dadurch sind sie sehr gut kartierbar und die Gletschertopographien können gut rekonstruiert werden. Für diesen Zeitraum ist auch die Verbreitung von Blockgletschern gut belegt. Die meisten von ihnen bildeten sich in den Gebieten, die nach dem Maximalvorstoß eisfrei wurden. Gelegentlich lassen sie sich mit Moränensystemen der zweiten Phase in Beziehung bringen. Dort, wo es die Topographie erlaubt, reichen sie bis etwa 1900 m hinab (Sailer und Kerschner 1999, Frauenfelder et al. 2001). 


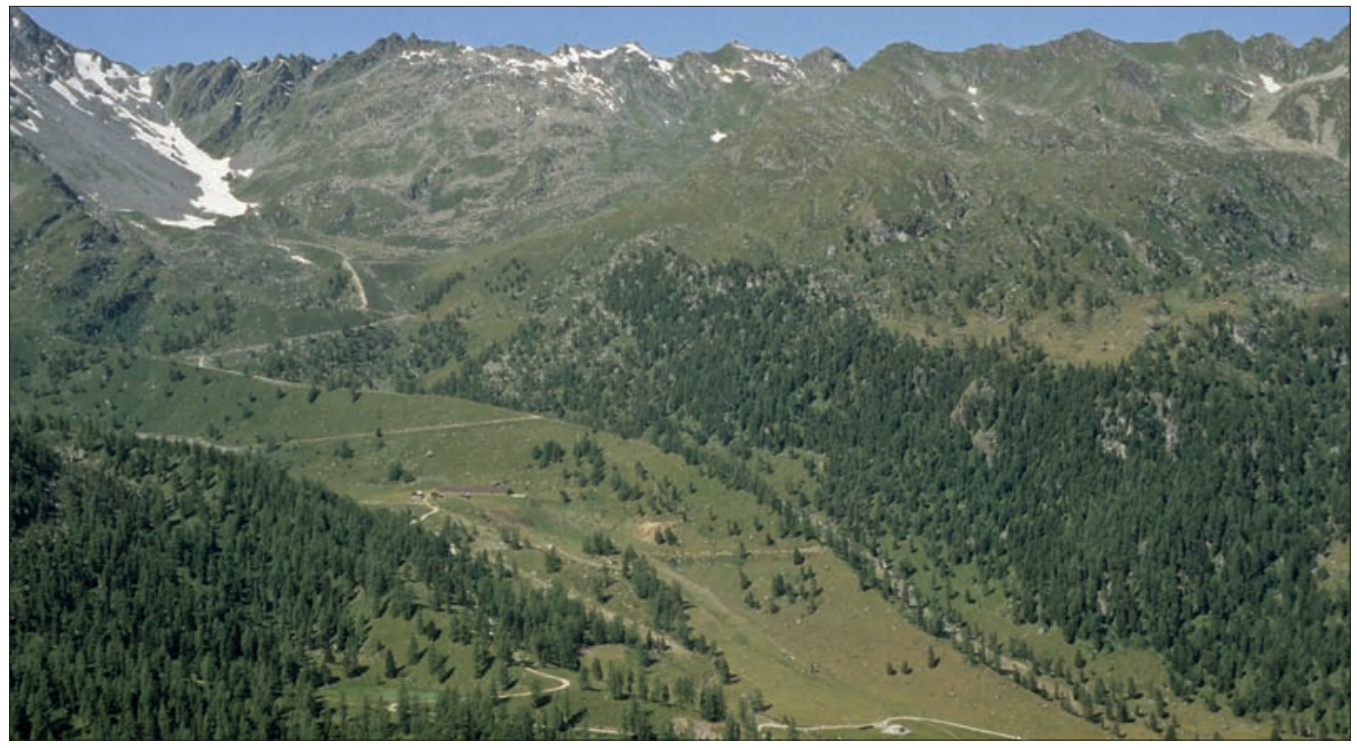

Abb. 2: Egesenzeitliche Ufermoräne „Grand Tôit", Val de Nendaz, Unterwallis (Foto H. Kerschner, 1981).

Fig. 2: Egesen lateral moraine (Gand Tôit, Val de Nendaz, Valais, Switzerland)(Photo H. Kerschner, 1981).

Die Stabilisierungsalter der Moränen des Maximalvorstoßes liegen im Bereich von

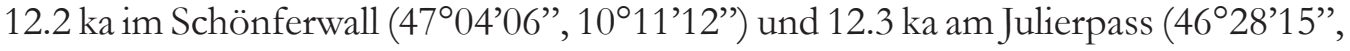
944’48”) (Ivy-Ochs et al. 1996, 2006), also etwa 400 - 500 Jahre nach dem Beginn der Klimaverschlechterung. Die Stabilisierung der jüngeren Moränen und der damit verbundenen Blockgletscher fällt mit dem Ende der Jüngeren Dryas um ca. $11.5 \mathrm{ka}$ zusammen (Ivy-Ochs et al. 2008).

Die Schneegrenzdepression des Egesen-Maximalstandes lag in den gut abgeschirmten Tälern der Zentralalpen bei etwa 200 m oder etwas darüber, während sie im Nordalpenraum bis gegen $400 \mathrm{~m}$ ging. Besonders tief lag die Schneegrenze dort, wo in tiefen, nach $\mathrm{N}-\mathrm{NW}$ gerichteten Tälern das Heranführen feuchter Luft in niedrigen Höhen möglich ist. Im einzelnen zeigt sie eine Abhängigkeit vom Großrelief der Alpen insofern, als sie auch im Zentralalpenraum in Paßlagen etwas tiefer liegt als in deren Nachbarschaft. Im Südalpenraum dürfte sie dort, wo die Täler gut abgeschirmt sind, ebenfalls im Bereich von $200 \mathrm{~m}$ gelegen haben, während sie in den gegen das Mittelmeer exponierteren Bereichen gegen $300 \mathrm{~m}$ ging. Für die jüngeren Abschnitte des Egesenstadiums fehlt noch ein systematischer Überblick; im zentralen Alpenraum dürfte ein Betrag von etwa $150 \mathrm{~m}$ ein guter Richtwert für die jüngsten Vorstöße sein. 
Klimawandel in Österreich

\section{Frühes Holozän}

Zwischen den innersten Moränen des Egesenstadiums und den Moränen der neuzeitlichen Größenordnung («1850er -Moränen») findet man vielerorts weitere Moränensysteme eingeschachtelt. Sie zeichnen Gletscherausdehnungen nach, die deutlich größer als um 1850 waren. Je nach den lokalen Verhältnissen sind sie von den innersten Egesenmoränen entweder deutlich abgesetzt oder erwecken eher den Eindruck eines kontinuierlichen, von Vorstößen unterbrochenen Rückzugs. An einer besonders schön entwickelten Stelle im Moostal in der Ferwallgruppe wurde dafür der Ausdruck «Kartell-Schwankung» geprägt (Fraedrich 1979). Die Datierung der Moräne (47 $\left.03^{\prime} 18^{\prime \prime}, 10^{\circ} 15^{\prime} 18^{\prime \prime}\right)$ ergab einen Mittelwert von 10.8 ka für ihre Stabilisierung (Sailer 2002, Ivy-Ochs et al. 2006b). Damit kommt für den Gletschervorstoß am ehesten die Präboreale Oszillation (11.3-11.15 ka: Björck et al. 1997; 11.4-11.1 ka: Schwander et al. 2000 ) als Auslöser in Frage. Der Zeitraum von 150 bzw. 300 Jahren, den die Präboreal Oszillation umfaßte, ist jedenfalls hinreichend lang, um die in Frage kommenden Gletschervorstöße zu verursachen. Die Schneegrenzdepression lag bei $120 \mathrm{~m}$. Eine ähnliche Situation im Langtauferstal (Südtirol) zeigt vergleichbare Alter. Allerdings kann man dort noch weitere Moränensysteme kartieren, und der Übergang zur neuzeitlichen Größenordnung ist fließend. Die Entwicklung von Blockgletschern (gefrorene, eishaltige Schuttmassen unterschiedlicher Herkunft) und die blockgletscherartige Überformung vieler Moränen zeigt, daß um diese Zeit noch Permafrost in tieferen Lagen als heute existieren konnte. Die Stabilisierung der Blockgletscher am Julierpaß vor 10.400 Jahren (Ivy-Ochs et

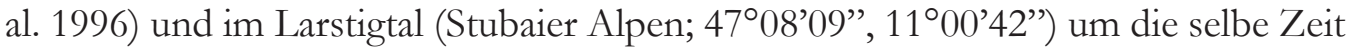
(Ivy-Ochs et al., im Druck) markiert das Ende des Zeitraumes, in dem die Gletscher deutlich größer als während der «Kleinen Eiszeit» waren, und in der Permafrost rund $200 \mathrm{~m}$ tiefer als im 20. Jahrhundert existieren konnte. Tatsächlich sollte das Ende dieser Phase bereits etwas früher erreicht worden sein, denn um etwa 10.500 Jahren vor heute waren die großen alpinen Gletscher bereits kleiner als heute (Nicolussi, Patzelt 2001, Joerin et al. 2008 ).

Ein Gletscherstand im oberen Kromertal (Silvrettagruppe; 46 54'38, 1002'14”) mit rund $80 \mathrm{~m}$ Schneegrenzdepression (Kromer - Stand; Gross et al. 1977) scheint in diesem Zusammenhang aus der Reihe zu fallen. Wegen der räumlichen Nähe zur Kartell - Lokalität und der Ähnlichkeit des Gletscherstandes wurden die Moränen datiert, was überraschenderweise ein mittleres Alter von 8.4 ka ergab (Kerschner et al. 2006). Damit käme eventuell der klimatisch instabile, etwas kühlere und feuchtere Zeitabschnitt (Central European cold phase 3: Haas et al 1998) als Auslöser in Frage, 
in dem der «8.2 ka-event» eingebettet ist (vgl. z.B. v. Grafenstein et al. 1998, Magny, Bégeot 2004, Tinner, Lotter 2001, Schmidt et al. 2004). Allerdings gibt es dafür in den Alpen keine weitere gletschergeschichtliche Bestätigung, vielmehr waren damals die bekannten Gletscherausmaße kleiner als um 1850 (Nicolussi, Patzelt 2001, Joerin et al. 2008). Der Fragenkreis um die zeitliche Stellung und damit auch der nach der klimatischen Bedeutung des Kromerstandes muß deshalb noch offen bleiben. Auch das genaue Alter der Venediger Schwankung (Patzelt \& Bortenschlager 1973) und dessen Stellung zum Kartell- und Kromerstand ist derzeit noch offen.

\section{Klimatische Interpretation}

Die klimageschichtliche Interpretation dieser Gletscherschwankungen baut darauf auf, daß Schwankungen der Gleichgewichtslinie unmittelbar klimatische Ursachen haben, die auch klimatologisch, d.h. in einem längeren zeitlichen Mittel (ca. 30-100 Jahre) betrachtet werden können. Der Zusammenhang zwischen Schwankungen der Schneegrenze (klimatologisches Mittel der Gleichgewichtslinie; Gross et al. 1977, Kerschner 1990) und Klimaschwankungen kann mit der Energie- und Massenbilanzgleichung untersucht werden (Kuhn 1981, 1989). Sie zeigt im Prinzip die Komplexität des Klimageschehens in seinem Einfluß auf Gletscher. In einer ersten Näherung kann man aber auch einfachere Verfahren verwenden. Sie bauen darauf auf, daß die Sommertemperatur an der Gleichgewichtslinie ein guter Parameter für alle Wärmeströme ist, die die Ablation steuern, während der Jahresniederschlag ein guter Parameter für die Akkumulation ist. Die statistischen Zusammenhänge zwischen diesen beiden Größen («P,T-Modelle») sind von verschiedenen Autoren für unterschiedliche Gebiete formuliert worden (vgl. Zusammenstellungen in Kerschner 2005, Kerschner und Ivy-Ochs 2007). Sie zeigen alle, daß der Niederschlag mit steigender Sommertemperatur an der Gleichgewichtslinie überproportional zunimmt. In ihrer Gültigkeit sind sie auf den Raum beschränkt, aus dem die Gletscher der Stichprobe stammen, also z.B. Norwegen. Im alpinen Raum hat sich die von Ohmura et al. (1992) aufgestellte Beziehung zwischen dem Niederschlag und der Sommertemperatur an der Gleichgewichtslinie, die auf einer weltweiten Stichprobe aufbaut, am besten bewährt. Wenn man die Klimadaten auf die Normalperiode 1931-60 bezieht, so ergibt die Gleichung von Ohmura et al. (1992) bei einer Sommertemperatur von $0^{\circ} \mathrm{C}$ einen von Niederschlag $830 \mathrm{~mm} / \mathrm{a}$, bei $2^{\circ} \mathrm{C}$ liegt er bei etwa $1500 \mathrm{~mm} / \mathrm{a}$ und bei $4^{\circ} \mathrm{C}$ bei rund $2200 \mathrm{~mm} / \mathrm{a}$. 
Für alle klimageschichtlichen Überlegungen ist es gerade in einer Zeit mit wechselnden klimatischen Verhältnissen wichtig, einen Bezugsrahmen („heute“) zu definieren. Hier wird die klimatische Normalperiode (-> von der WMO definierte 30 jährige Zeiträume) 1931-60 verwendet, einerseits, weil sie den klimatischen Hintergrund für die Gletscherinventare der späten Sechziger- und Siebzigerjahre liefert, und andererseits, weil sie für das gesamte 20. Jahrhundert typisch ist.

Wenn nun die Änderung der Sommertemperatur aus anderen, möglichst gut synchronisierten Proxydaten (z.B. Waldgrenzschwankungen oder paläolimnologische Daten: vgl. z.B. Ammann et al. 2000, Magny et al. 2007) bekannt ist, kann mit einem der angeführten Verfahren aus der Schneegrenzdepression die Änderung des Niederschlags abgeleitet werden. Dabei sind die Waldgrenzschwankungen besonders interessant, da die Waldgrenze ein guter Indikator für die Sommertemperatur auf der selben Zeitskala wie die Ablationsperiode der Gletscher ist (Körner 2007). Änderungen des Temperaturfeldes sind zumindest in einem derart kleinen Gebiet wie den Alpen räumlich etwa konstant. Für einen gegebenen Zeitraum muß daher bei einer sehr starken Schneegrenzdepression mehr Niederschlag zur Verfügung gestanden haben, bei einer geringen weniger.

Die einfache Handhabung, in der Praxis erfolgt die Berechnung mit EXCELTabellen, erlaubt es, verschiedene Szenarien zu berechnen und damit Bandbreiten für die möglichen Änderungen abzuschätzen (z.B. Kerschner und Ivy-Ochs 2007). Wegen der großen Stichprobe ist es auf diese Weise möglich, für die Zeit des Egesen-Maximalstandes (erste Hälfte der Jüngeren Dryas) die Ergebnisse auch in Kartenform darzustellen (Kerschner et al. 2000; Abb. 3) und regelmäßig nachzuführen. Die Verläßlichkeit derartiger Karten ist im einzelnen schwierig abzuschätzen; der Vergleich von Szenarien zeigt aber, daß die prinzipiellen Strukturen auch bei veränderten Annahmen erhalten bleiben. Dazu gehört in erster Linie eine verstärkte Trockenheit in den gut abgeschirmten Tälern der inneren Alpen und ein feuchter Alpennordsaum. In den Ötztaler Alpen und im oberen Inngebiet war der Niederschlag etwa 20 - 30\% geringer als heute, während er am Alpennordsaum ungefähr gleich wie heute war. Diese Werte gelten für eine Sommertemperaturdepression von 3.5 Grad. Verschiedene weitere Überlegungen (Kerschner, Ivy-Ochs 2007) zeigen, daß die Sommertemperaturdepression in der ersten Hälfte der Jüngeren Dryas nicht über $5 \mathrm{Grad}$ hinausgegangen sein kann. In diesem Fall hätten die trockenen Gebiete nur mehr die Hälfte des heutigen Niederschlags bekommen, und auch der Alpennordsaum wäre etwas trockener als heute gewesen. Damit ergibt sich für den ersten Abschnitt der Jüngeren Dryas jedenfalls ein stärkerer Niederschlagsgradient vom 
Alpennordsaum in das Alpeninnere, der in den meisten Fällen an charakteristische topographische Barrieren gebunden ist. Die relativ feuchten Gebiete greifen dort am weitesten in das Alpeninnere hinein, wo Täler mit tiefliegenden Talböden direkt nach Norden und Nordwesten hinausführen (Hertl und Kerschner 2001). Auch die zum Mittelmeer hin exponierten Gebiete dürften relativ feucht gewesen sein (Federici et al. 2008).

Die späteren Abschnitte des Egesenstadiums sind durch deutlich kleinere Gletscher und die Entwicklung großer Blockgletschersysteme gekennzeichnet (Sailer, Kerschner 1999 und dort zitierte Literatur). Dabei ist vor allem der Rückgang zwischen der ersten und der zweiten Phase des Egesenstadiums markant. Insgesamt weist dieses Bild auf trockenere Verhältnisse hin, wenn man davon ausgeht, daß die Depression der Sommertemperatur etwa ähnlich wie in der ersten Phase war. Die Blockgletscher reichen in günstigen Positionen bis zu $600 \mathrm{~m}$ tiefer als die heute aktiven Blockgletscher hinab. In den gleichen Gebieten beträgt die Schneegrenzdepression nur etwa 200 bis knapp $300 \mathrm{~m}$. Dementsprechend sollte die Erniedrigung der Jahresmitteltemperatur, die für die Blockgletscherverbreitung maßgebend ist, größer gewesen sein als die der Sommermonate. Insgesamt entspricht dieses Bild gut den Ergebnissen aus pollenanalytischen und paläolimnologischen Untersuchungen, die im Alpenraum auf eine Zweiteilung der Jüngeren Dryas in einen kühl-feuchten und kalt-trockenen Abschnitt mit einer wärmeren Zwischenphase hinweisen (z.B. Welten 1982, Schmidt 1984, Schmidt et al. 2002).

Für das Kartellstadium im frühen Präboreal lassen sich noch keine detaillierten Angaben machen. Geht man davon aus, daß die Präboreale Oszillation zum ersten Mal nach dem Ende der Jüngeren Dryas wieder kurzfristig feuchtere Verhältnisse brachte (Schmidt et al. 2004, Magny et al. 2007), so würde man in der Ferwallgruppe mit einer Sommertemperaturdepression von etwa $1.5^{\circ}$ bis $2{ }^{\circ} \mathrm{C}$ gegen «heute» das Auslangen finden.

Die Hauptschwierigkeit bei dieser Vorgangsweise besteht in einer möglichst genauen Synchronisation der Befunde, die optimal in einer zeitlichen Größenordnung von 10 bis 100 Jahren gegeben sein müßte, also im Bereich der Reaktionszeit der Gletscher. Davon ist man jedoch weit entfernt, sodaß man mit einem «Verschleifen» der Ergebnisse rechnen muß. Dadurch werden die Extreme gekappt; die tatsächlichen Verhältnisse während der Jüngeren Dryas könnten also auf einer Zeitskala von wenigen Jahrzehnten extremer gewesen sein als es den Anschein hat. Diesen Umstand muß man auch bedenken, wenn man eine stratigraphische Zuordnung sehr kleiner Gletscher mit kurzer Anpassungszeit durchführen will. 


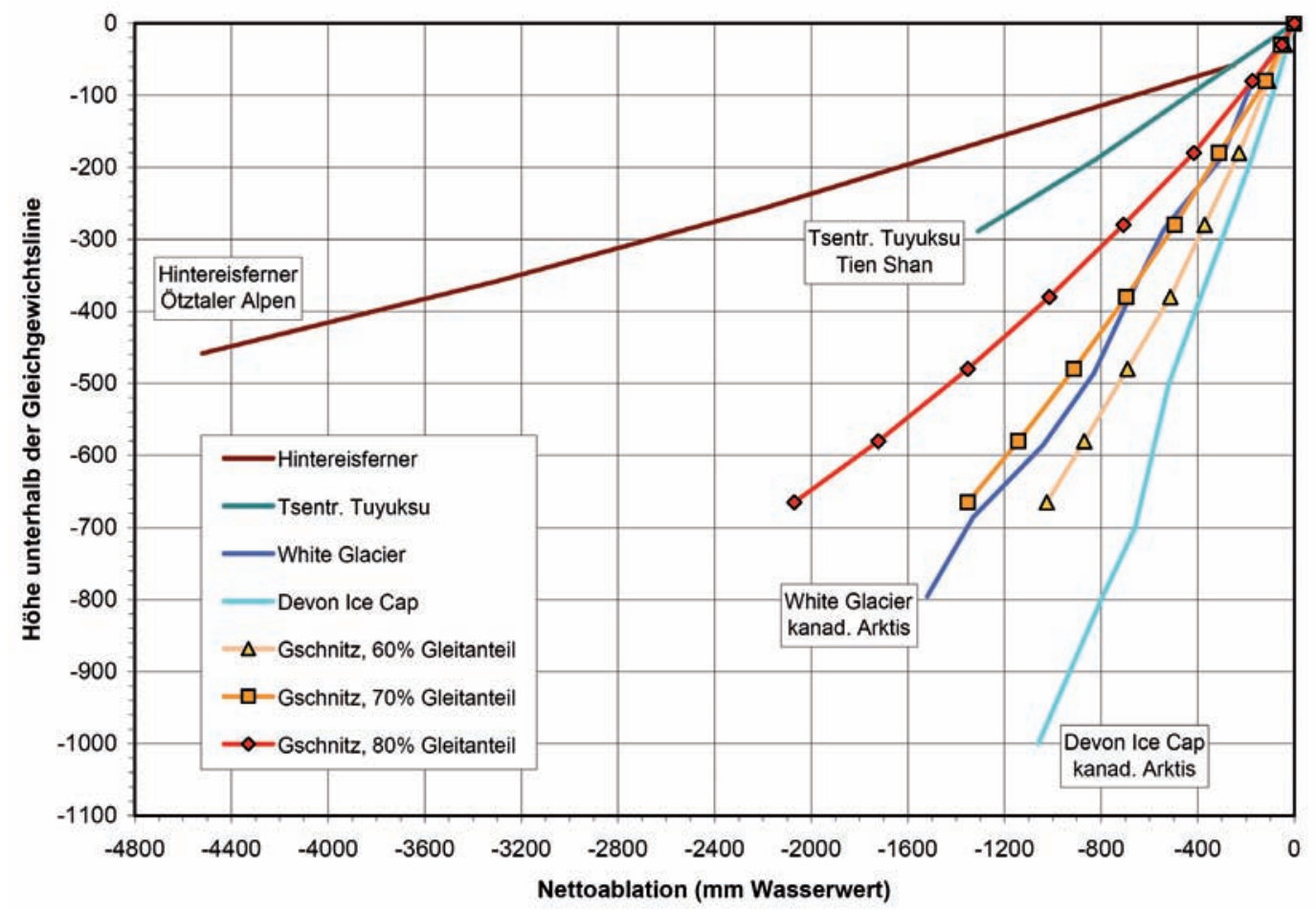

Abb. 3: Ablationsgradienten entlang der Zunge des Gschnitzgletschers im Gschnitztal, Tirol (verändert nach Ivy-Ochs et al. 2006a) im Vergleich zu Gletschern aus anderen Gebieten.

Fig. 3: Ablation gradients along the tongue of the Gschnitz glacier in Gschnitz Valley, Tyrol (redrawn from Ivy-Ochs et al. 2006a) in comparison to glaciers from other regions.

Während die Kombination von gletschergeschichtlichen Befunden und biologischen Proxydaten für die Jüngere Dryas noch einigermaßen gut funktioniert, ist die Rekonstruktion von Sommertemperaturen für das ältere Spätglazial mit Hilfe biologischer Proxydaten durch das weitgehende Fehlen entsprechender Fossilien limitiert. Für den Gschnitzgletscher an der Typlokalität im Gschnitztal kann man über ein einfaches Fließmodell den Eisabfluß berechnen, da das Tal im kritischen Bereich eine sehr einfache Topographie aufweist. Daraus kann man den Ablationsgradienten (Abnahme der Ablation mit der Höhe) entlang der Gletscherzunge (Abb. 3) rekonstruieren. Der Eisdurchfluß im Querschnitt an der Gleichgewichtslinie muß dann gleich der Nettoakkumulation sein. Damit läßt sich der Niederschlag im Akkumulationsgebiet und in weiterer Folge die Sommertemperatur an der Gleichgewichtslinie abschätzen (Kerschner et al. 1999, Ivy-Ochs et al. 2006a). Da der Gletscher insgesamt 
sehr träge war, kam er mit etwa einem Drittel bis höchstens der Hälfte der heutigen Niederschläge aus, die Sommertemperatur war rund $10 \mathrm{Grad}$ niedriger als heute. Im Inntal erreichten sie kaum $9{ }^{\circ} \mathrm{C}$. Diese Werte stimmen gut mit der aus kalibrierten Kieselalgen rekonstruierten Wassertemperatur des Längsees für die Zeit des Gschnitzstadials überein (Schmidt et al., in diesem Band). Die heutigen Analoga für derartige Gletscher findet man am ehesten in der Arktis oder in den sehr trockenen Hochgebirgen in Zentralasien; der White Glacier auf Axel Heiberg Island in der kanadischen Arktis (Cogley et al. 1996) ist ein gut vergleichbares rezentes Beispiel für einen derartigen Gletscher.

Weitere Informationen kann man aus der Modellierung der Ablationsgradienten mit der Energie- und Massenbilanzgleichung gewinnen, wie sie von Kaser (2001) für tropische Gletscher durchgeführt wurde. Die klimatologisch modellierten Ablationsgradienten können dann so angepaßt werden, daß sie denen entsprechen, die aus dem Fließmodell abgeleitet wurden. Erste Versuche zeigen, daß während des Gschnitzstadiums der Sommer mit Ablationsbedingungen in $1900 \mathrm{~m}$ Seehöhe kaum 50 Tage gedauert haben kann, während man heute in diesem Gebiet mit etwa 100 Tagen in $2750 \mathrm{~m}$ Höhe rechnen kann. An der Gletscherzunge in der Höhe der Ortschaft Trins (1200 m) war nur an rund 100 - 120 Tagen Eisschmelze möglich. Auch Gradtagmodelle (Hughes, Braithwaite 2008) könnten für die klimageschichtliche Untersuchung dieser Gletscherstände interessante weitere Aufschlüsse liefern.

Eine weitere, eher qualitative Möglichkeit der klimageschichtlichen Interpretation, bietet die basale Scherspannung an der Basis der Gletscherzunge, die vom Oberflächengefälle und der Eisdicke abhängt. Bei heutigen, gut ernährten alpinen Gletschern liegt sie im Bereich von 100 Kilopascal, nur in sehr steilen Partien liegt sie auf kurzen Strecken darüber (vgl. Maisch, Haeberli 1982). Diese Werte wurden auch für die Gletscher der Jüngeren Dryas erreicht, die in ihrer Charakteristik heutigen Gletschern vergleichbar waren. Im Gegensatz dazu wiesen die Gletscher vor dem Beginn des spätglazialen Interstadials sehr flache Zungen auf, deren Scherspannung im Bereich von $70 \mathrm{kPa}$ und darunter lagen. Derartige Werte sind für schlecht ernährte Gletscher unter sehr kalten Klimabedingungen typisch. 


\section{Scblußfolgerungen}

Nach dem heutigen Kenntnisstand kann man die späteiszeitliche Gletschergeschichte grob in zwei Abschnitte gliedern. Zwischen dem hocheiszeitlichen Maximum um ca. $21 \mathrm{ka}$ und dem Beginn des spätglazialen Interstadials um ca. $14.7 \mathrm{ka}$ existierten die Gletscher in einem wohl sehr kalten und überwiegend trockenen Klima. Dies begünstigte eine langsame Reaktion der Gletscher auf Klimaschwankungen, wovon der «Heinrich-1 ice rafting event» (Grönland Stadial 2a) wohl die bedeutendste Klimaverschlechterung war. Er verursachte den Gletschervorstoß des Gschnitzstadiums (ca. $16 \mathrm{ka}$ ). Aus den glaziologischen Befunden läßt sich für das Gschnitzstadium eine Sommertemperaturdepression von etwa $10^{\circ} \mathrm{C}$ rekonstruieren, die jährliche Niederschlagssumme erreichte im Zentralalpenraum nur etwa $1 / 3$ bis höchstens der Hälfte der heutigen Mengen. Die räumliche Verbreitung des Gschnitzstadiums ist nur lückenhaft bekannt. Je nach dem Vergletscherungsgrad eines Gebietes können mit einer Schneegrenzdepression von etwa $700 \mathrm{~m}$ gegen $1850 \mathrm{sehr}$ unterschiedlich große Gletscher entstehen. Zudem scheint es, als ob auch während des Gschnitzstadiums eine räumliche Variabilität der Schneegrenzdepression bestand. Aus klimatologischer Sicht ist eine großräumig konstante Schneegrenzdepression jedenfalls eher unwahrscheinlich.

Zwischen dem Gschnitzstadium und dem Beginn des spätglazialen Interstadials (Zeitraum von ca. 1000 Jahren) sind noch Moränen weiterer, kleinerer Vorstöße einzureihen, die in der ostalpinen Stratigraphie dem Clavadel/Sendersstadium und dem Daunstadium zugeordnet werden. Ihre zeitliche Zuordnung ist noch nicht genau bekannt; in nächster Zeit können hier jedoch wesentliche Fortschritte erwartet werden(Boehlert et al. 2008).

Während des spätglazialen Interstadials hat es sicher auch Gletscherschwankungen gegeben, deren Spuren jedoch von den späteren Vorstößen, vor allem jenen der Jüngeren Dryas, ausgelöscht wurden. Die mehrphasige Jüngere Dryas verursachte die vielgliedrige Serie des Egesenstadiums, dessen spätere Abschnitte durch Eisrückzug und großfächige Blockgletscherentwicklungen bei weitverbreitetem alpinem Permafrost gekennzeichnet waren. Nimmt man eine aus der Waldgrenzdepression abgeleitete Erniedrigung der Sommertemperatur von $3.5^{\circ} \mathrm{C}$ an, so ergibt sich daraus, daß der Alpennordsaum ähnlich feucht wie heute oder möglicherweise auch etwas niederschlagsreicher war, während die gut abgeschirmten Alpentäler bis zu 30\% trockener als heute waren. Die Erniedrigung der Jahresmitteltemperatur, die sich aus der Höhe der Blockgletscher ableiten läßt, war etwas größer als die der Sommertemperatur. 


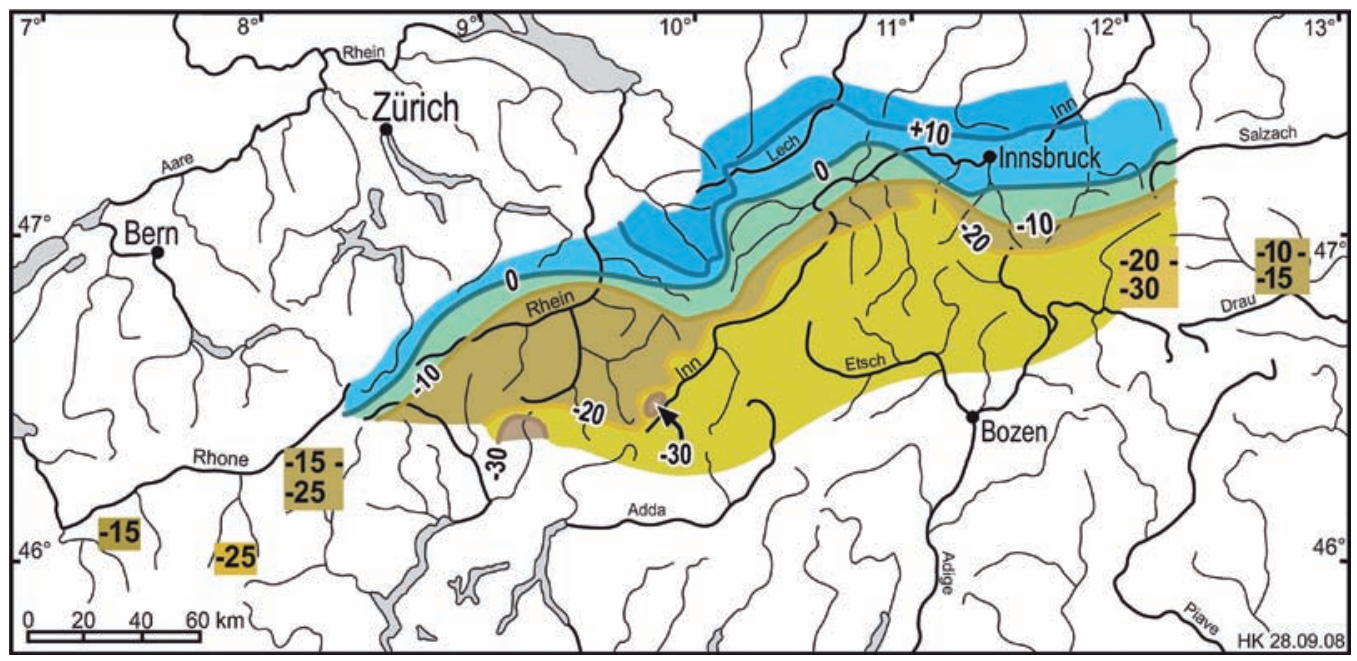

Abb. 4: Karte der Niederschlagsänderung während des Egesenmaximums im mittleren Alpenraum (nachgeführt nach Kerschner 2005).

Fig. 4: Map of precipitation changes during the Egesen maximum in the Central Alps (updated from Kerschner 2005).

Die Ausdehnung der egesenzeitlichen Vergletscherung ist zwar entlang der zentralen Alpenachse zwischen Brennerpaß und Mont Blanc gut bekannt, außerhalb davon sind die Befunde jedoch lückenhaft (vgl. Abb. 4). Mitunter wird man sie auch kaum je schließen können, wenn auf Grund der topographischen Verhältnisse die Moränen fehlen. Das betrifft vor allem den nördlichen Außensaum der Alpen, aber auch südlich des Alpenhauptkammes und in den Westalpen sind die Lücken größer als die Kenntnisse. Besonderes Augenmerk verdienen hier Gebiete mit kleinen Gletschern, die auf Klimaschwankungen rasch reagieren. In der Vergangenheit wurde bei der Korrelation von Gletscherständen mit dem Egesenstadium ein (zu ?) hohes Gewicht auf die Schneegrenzdepression gelegt, deshalb müßten einige ältere Kartierungen wieder überprüft und gegebenenfalls neu bewertet werden.

Die genaue zeitliche Einstufung und Synchronisation der frühholozänen Moränenstaffeln, die von jenen der Spätphasen des Egesenstadiums abgegrenzt werden können und außerhalb des neuzeitliche Hochstandes (1850) liegen, ist derzeit nur für einzelne Gletscher möglich. Das umfaßt in erster Linie die Gletscherstände, die traditionell als «Kromer/Kartell» zusammengefaßt wurden. Während das Moränenalter des Kartell-Vorstoßes auf 10.8 ka bestimmt wurde, liegt es beim Kromer-Vorstoß bei $8.4 \mathrm{ka}$. Da es sich dabei um Einzelbefunde handelt, ist eine Zusammenschau und Parallelisierung mit den bekannten Klimaschwankungen im 
frühen Holozän zwischen der Präborealen Oszillation und dem «8.2 ka - event» noch nicht zufriedenstellend möglich. Damit läßt sich auch nicht sagen, ob diese Gletschervorstöße allein durch Abkühlungsphasen verursacht wurden, oder ob eine Zunahme der Niederschläge nach dem Ende der Jüngeren Dryas wesentlich waren.

Die Fortschritte, die die absolute Datierung von Moränen in den letzten Jahren gebracht hat, dürfen jedoch nicht darüber hinwegtäuschen, daß nach wie vor lange Zeitabschnitte und auch viele räumliche Muster noch weitgehend unbekannt sind. Beim gegenwärtigen Stand lassen sich für die zukünftige gletschergeschichtliche Forschung vor allem zwei interessante Zeiträume identifizieren. Dabei handelt es sich zum einen um das «ältere Spätglazial», also den Zeitraum zwischen dem endgültigen Rückzug der eiszeitlichen Vorland- und Talgletscher und dem Beginn des spätglazialen Interstadials. Der zweite Zeitabschnitt umfaßt das frühe Holozän zwischen dem Ende der Jüngeren Dryas vor 11.5 ka und dem endgültigen Verschwinden der Reste der Inlandseisgebiete in Nordamerika und Skandinavien um etwa 8 ka. Dieser Zeitabschnitt brachte möglicherweise eine zeitlich bunte und regional stark differenzierte Reaktion der alpinen Gletscher in einem noch relativ instabilen Klima. Dazu kommt schließlich noch das Verdichten der Kartierungen und damit der Übergang von punkthaften zu flächenhaften Informationen.

\section{Dank}

Große Teile der Untersuchungen, die dieser Zusammenfassung zugrunde liegen, wurden durch die FWF-Projekte P12600-GEO und P15108-N06 wesentlich gefördert.

\section{Literatur}

Ammann, B., Birks, H.J.B., Brooks, S.J., Eicher, U., von Grafenstein, U., Hofmann, W., Lemdahl, G., Schwander, J., Tobolski, K., Wick, L., 2000. Quantification of biotic responses to rapid climatic changes around the Younger Dryas - a synthesis. Palaeogeography, Palaeoclimatology, Palaeoecology 159, 313-347.

Becker, B., Kromer, B., 1986. Extension of the Holocene dendrochronology in Preboreal pine series, 8,800 to 10,100 BP. Radiocarbon 28, 961-67.

Becker, B., Kromer, B., 1993, The continental tree-ring record — absolute chronology, ${ }^{14} \mathrm{C}$ calibration and climatic change at $11 \mathrm{ka}$. Palaeogeography, Palaeoclimatology, Palaeoecology 103, 67-71. 
Björck, S., Rundgren, M., Ingólfsson, Ó., Funder, S., 1997. The Preboreal oscillation around the Nordic Seas: terrestrial and lacustrine responses. Journal of Quaternary Science 12, 455-465.

Björck, S., Walker, M.C., Cwynar, L.C.; Johnsen, S., Knudsen, K.-L., Lowe, J.J., Wohlfahrt, B., INTIMATE members. 1998. An event stratigraphy for the Last Termination in the North Atlantic region based on the Greenland ice-core record: a proposal by the INTIMATE group. Journal of Quaternary Science 13(4) 283-292

Boehlert, R., Egli, M., Maisch, M., Brandová, D., Ivy-Ochs, S., Haeberli, W., 2008. Landscape evolution in the Albula region (Grisons, eastern Switzerland) during the Lateglacial and early Holocene. Geophysical Research Abstracts 10, EGU2008-A-08608.

Cogley, J.G., Adams, W.P., Ecclestone, M.A., Jung-Rothenhäusler, F., Omanney, C.S.L., 1996. Mass balance of White Glacier, Axel Heiberg Island, N.W.T., Canada, 1960-91. Journal of Glaciology 42(142), 548-563.

Coutterand, S., Nicoud, G., 2005. Les stades de retrait du glacier de l'Arve entre le verrou de Cluses et l'ombilic de Chamonix au cours du Tardiglaciaire (Vallée de l'Arve, Haute-Savoie). Quaternaire 16, 85-94, 2005.

Federici, P.R., Granger, D.E., Pappalardo, M., Ribolini, A., Spagnolo, M., Cyr, A.J., 2008. Exposure age dating and equilibrium line altitude reconstruction of an Egesen moraine in the Maritime Alps, Italy. Boreas 37, 245-253.

Fraedrich, R., 1979. Spät-und postglaziale Gletscherschwankungen in der Ferwallgruppe (Tirol/ Vorarlberg). Düsseldorfer Geographische Schriften 12, 1-161.

Frauenfelder, R., Haeberli, W., Hoelzle, M., Maisch, M., 2001. Using relict rockglaciers in GIS-based modelling to reconstruct Younger Dryas permafrost distribution patterns in the Err-Julier area, Swiss Alps. Norsk Geografisk Tidsskrift, Norwegian Journal of Geography 55, 195-202.

Gosse, J., Phillips, F. M., 2001. Terrestrial in situ cosmogenic nuclides: theory and application. Quaternary Science Reviews 20, 1475-1560.

von Grafenstein, U., Erlenkeuser, H., Müller, J., Jouzel, J. Johnsen, S., 1998. The cold event 8200 years ago documented in oxygen isotope records of precipitation in Europe and Greenland. Climate Dynamics 14, 73-81.

Gross, G., Kerschner, H., Patzelt, G., 1977. Methodische Untersuchungen über die Schneegrenze in alpinen Gletschergebieten. Zeitschrift für Gletscherkunde und Glazialgeologie 12, 223-251.

Haas, J.N., Richoz, I., Tinner, W. and Wick, L., 1998: Synchronous Holocene climatic oscillations recorded on the Swiss Plateau and at timberline in the Alps. The Holocene 8, 301-309.

Hemming S. 2004. Heinrich events: massive late Pleistocene detritus layers of the North Atlantic and their global climate imprint. Reviews of Geophysics 42, 1-43.

Heuberger, H., 1966. Gletschergeschichtliche Untersuchungen in den Zentralalpen zwischen Sellrain und Ötztal. Wissenschaftliche Alpenvereinshefte 20. Universitätsverlag Wagner, Innsbruck.

Hertl, A., 2001. Untersuchungen zur spätglazialen Gletscher- und Klimageschichte derösterreichischen Silvrettagruppe. Natwiss. Diss. Universität Innsbruck, 313 S.

Hoinkes, H., 1970. Methoden und Möglichkeiten von Massenhaushaltsstudien auf Gletschern. Ergebnisse der Meßreihe Hintereisferner (Ötztaler Alpen) 1953 - 1968. Zeitschrift für Gletscherkunde und Glazialgeologie 6, 37-90.

Hughes, Ph., Braithwaite, R., 2008. Application of a degree-day model to reconstruct Pleistocene glacial climates. Qaternary Research 69, 110-116. 


\section{Klimawandel in Österreich}

Ivy-Ochs, S., Schlüchter, C., Kubik, P.W., Synal, H.-A., Beer, J., Kerschner, H., 1996. The exposure age of an Egesen moraine at Julier Pass, Switzerland, measured with the cosmogenic radionuclides ${ }^{10} \mathrm{Be},{ }^{26} \mathrm{Al}$ and ${ }^{36} \mathrm{Cl}$. Eclogae geologicae Helvetiae 89, 1049-1063.

Ivy-Ochs, S., Schäfer, J., Kubik, P.W., Synal, H.-A., Schlüchter, C., 2004. The timing of deglaciation on the northern Alpine foreland (Switzerland). Eclogae geologicae Helvetiae 97, 47-55.

Ivy-Ochs, S., Kerschner, H., Kubik, P.W., Schlüchter, C., 2006a. Glacier response in the European Alps to Heinrich event 1 cooling: the Gschnitz stadial. Journal of Quaternary Science 21, 115-130.

Ivy-Ochs, S., Kerschner, H., Reuther, A., Maisch, M., Sailer, R., Schaefer, J., Kubik, P.W., Synal, H.A., Schlüchter, C., 2006b. The timing of glacier advances in the northern European Alps based on surface exposure dating with cosmogenic ${ }^{10} \mathrm{Be},{ }^{26} \mathrm{Al},{ }^{36} \mathrm{Cl}$, and ${ }^{21} \mathrm{Ne}$. In: Siame LL, Bourlès DL, Brown ET (eds.): In Situ-Produced Cosmogenic Nuclides and Quantification of Geological Processes: Geological Society of America Special Paper 415, 43-60.

Ivy-Ochs, S., Kerschner, H., Schlüchter, C., 2007. Cosmogenic nuclides and the dating of glacier variations: The Alpine perspective. Quaternary International 164/165, 53-63.

Ivy-Ochs, S., Kerschner, H., Reuther, A., Preusser, F., Heine, K., Maisch, M., Kubik, P.W., Schlüchter, C., 2008. Chronology of the last glacial cycle in the Northern European Alps. Journal of Quaternary Science 23(6-7), 559-573.

Ivy-Ochs, S., Kober, F., 2008. Surface exposure dating with cosmogenic nuclides. Eiszeitalter und Gegenwart 57, 157-189.

Ivy-Ochs, S., Kerschner, H., Maisch, M., Christl, M., Kubik, P.W., Schlüchter, C., im Druck. ${ }^{10}$ Be surface exposure ages from rock glaciers in Larstigtal (Tyrol, Austria): Implications for the timing of latest Pleistocene and Holocene glacier variations in the European Alps. Quaternary Science Reviews.

Joerin, U.E., Nicolussi, K., Fischer, A., Stocker, T.F., Schlüchter, C., 2008. Holocene optimum events inferred from subglacial sediments at Tschierva Glacier, Eastern Swiss Alps. Quaternary Science Reviews 27, 337-350.

Kaser, G., 2001. Glacier-climate interaction at low latitudes. Journal of Glaciology 47, 195-204.

Kerschner, H., 1986. Zum Sendersstadium im Spätglazial der nördlichen Stubaier Alpen, Tirol. Zeitschrift für Geomorphologie Supplementband 61, 65-76.

Kerschner, H., 1993. Späteiszeitliche Gletscherstände im südlichen Karwendel bei Innsbruck, Tirol. Innsbrucker Geographische Studien 20, 47-55.

Kerschner, H., 2005. Glacier-climate models as palaeoclimatic information sources: Examples from the Alpine Younger Dryas period. In: U. M. Huber, H. K. M. Bugmann and M. A. Reasoner (eds.): Global Change and Mountain Regions (A State of Knowledge Overview), Dordrecht: Springer, 73-82 (Advances in Global Change Research 23).

Kerschner, H., Ivy-Ochs, S., Schlüchter, C., 1999. Paleoclimatic interpretation of the early late-glacial glacier in the Gschnitz valley, Central Alps, Austria. Annals of Glaciology 28, 135-140.

Kerschner, H., Kaser, G., Sailer, R., 2000. Alpine Younger Dryas glaciers as paleo-precipitation gauges. Annals of Glaciology 31, 80-84.

Kerschner, H., Hertl, A., Gross, G., Ivy-Ochs, S., Kubik, P.W., 2006. Surface exposure dating of moraines in the Kromer valley (Silvretta Mountains, Austria) - evidence for glacial response to the $8.2 \mathrm{ka}$ event in the Eastern Alps. The Holocene 16, 7-15. 
Kerschner, H., Ivy-Ochs, S., 2007. Palaeoclimate from glaciers: Examples from the Eastern Alps during the Alpine Lateglacial and early Holocene. Global and Planetary Change 60, 58-71.

Kerschner, H., Ivy-Ochs, S., Schlüchter, C., 2008. Gletscher und Klima im Ostalpenraum zwischen 16.000 und 11.000 Jahren vor heute. Abhandlungen der Geologischen Bundesanstalt 62, 165-168 (DEUQUA Wien 2008).

Klasen, N., Fiebig, M., Preusser, F., Reitner, J.M., Radtke, U., 2007. Luminescence dating of proglacial sediments from the Eastern Alps. Quaternary International 164/165, 21-32.

Körner, C., 2007. Climatic treelines: Conventions, global patterns, causes. Erdkunde 61, 316-324.

Kuhn, M., 1981. Climate and Glaciers. International Association of Hydrological Sciences Publication 131, 3-20.

Kuhn, M., 1989. The response of the equilibrium line altitude to climatic fluctuations: theory and observations. In: J. Oerlemans (Ed.), Glacier Fluctuations and Climatic Change. Kluwer, Dordrecht, pp. 407-417.

Magny, M., Bégeot, C., 2004. Hydrological changes in the European midlatitudes associated with freshwater outbursts from Lake Agassiz during the Younger Dryas event and the early Holocene. Quaternary Research 61, 181-192.

Magny, M., Vannière, B., de Beaulieu, J.-L., Bégeot, C., Heiri, O., Millet, L., Peyron, O., WalterSimmonet, A.-V., 2007. Early-Holocene climatic oscillations recorded by lake-level fluctuations in west-central Europe and in central Italy. Quaternary Science Reviews 26, 1951-1964.

Maisch, M., 1981. Glazialmorphologische und gletschergeschichtliche Untersuchungen im Gebiet zwischen Landwasser-und Albulatal. (Kt. Graubünden, Schweiz.) Physische Geographie 3, 216S.

Maisch M., 1982. Zur Gletscher- und Klimageschichte des alpinen Spätglazials: Geographica Helvetica 37, 93-104.

Maisch, M., Haeberli, W., 1982. Interpretation geometrischer Parameter von Spätglazialgletschern im Gebiet Mittelbünden, Schweizer Alpen. In Beiträge zur Quartärforschung in der Schweiz, Gamper M (ed.). Physische Geographie 1, 111-126.

Nicolussi, K., Patzelt, G., 2001. Untersuchungen zur holozänen Gletscherentwicklung von Pasterze und Gepatschferner (Ostalpen). Zeitschrift für Gletscherkunde und Glazialgeologie 36, 1-87.

Ohlendorf, C. 1998. High Alpine lake sediments as chronicles for regional glacier and climate history in the Upper Engadine, southeastern Switzerland. Diss. ETH No. 12705, Shaker Verlag, Aachen.

Ohmura, A., Kasser, P., Funk, M., 1992. Climate at the equilibrium line of glaciers. Journal of Glaciology 38, 397-411.

Patzelt, G., 1972. Die spätglazialen Stadien und postglazialen Schwankungen von Ostalpengletschern: Berichte der Deutschen Botanischen Gesellschaft 85, 47.

Patzelt, G., Bortenschlager, S., 1973. Die postglazialen Gletscher- und Klimaschwankungen in der Venedigergruppe (Hohe Tauern, Ostalpen). Zeitschrift für Geomorphologie NF, Suppl. Band 16, 25-72.

Penck A, Brückner E., 1901/1909. Die Alpen im Eiszeitalter. Tauchnitz: Leipzig.

Poscher, G., 1993, Neuergebnisse der Quartärforschung in Tirol. In: Geologie des Oberinntaler Raumes - Schwerpunkt Blatt 144 Landeck. Arbeitstagung der Geologischen Bundesanstalt 1993. Geologische Bundesanstalt Wien, 7-27. 


\section{Klimawandel in Österreich}

Reitner, J., 2005. Quartärgeologie und Landschaftsentwicklung im Raum Kitzbühel-St. Johann i.T.Hopfgarten (Nordtirol) vom Riss bis in das Würm-Spätglazial (MIS 6-2). Natwiss. Diss, Universität Wien.

Reitner, J., 2007. Glacial dynamics at the beginning of Termination 1 in the Eastern Alps and their stratigraphic implications. Quaternary International 164/165, 64-84.

Sailer, R., 2002. Späteiszeitliche Gletscherstände in der Ferwallgruppe. Natwiss.Diss, Universität Innsbruck.

Sailer, R., Kerschner, H., 1999. Equilibrium line altitudes and rock glaciers in the Ferwall Group (Western Tyrol, Austria) during the Younger Dryas cooling event. Annals of Glaciology 28, 135-140.

Schmidt R., 1984. Grundzüge der spät- und postglazialen Vegetations- und Klimageschichte des Salzkammergutes (Österreich) aufgrund palynologischer Untersuchungen von See- und Moorprofilen. Mitt. Komm. Quartärforsch. Österr. Akademie der Wissenschaften.

Schmidt, R., Psenner, R., Müller, J., Indinger, P., Kamenik, C., 2002. Impact of late glacial climate variations on stratification and trophic state of the meromictic lake Längsee (Austria): validation of a conceptual model by multi-proxy studies. Journal of Limnology 61, 49-60.

Schmidt, R., Kamenik, C., Kaiblinger, C., Hetzel, M., 2004. Tracking Holocene environmental changes in an alpine lake sediment core: application of regional diatom calibration, geochemistry, and pollen. Journal of Paleolimnology 32, 177-196.

Schoeneich, P., 1998. Le retrait glaciaire dans les vallées des Ormonts, de l'Hongrin et de l'Etivaz (Préalpes vaudoises). Institut de géographie - Travaux et recherches 14, Lausanne.

Studer, M., 2005. Gletschergeschichtliche Untersuchungen und geomorphologische Kartierung im Raum Maloja- Val Forno. Ein Beitrag zur regionalen Landschaftsgeschichte. Diplomarbeit Geographisches Institut der Universität Zürich.

Tinner, W., Lotter, A.F., 2001. Central European vegetation response to abrupt climate change at 8.2 ka. Geology 29, 551-554.

van Husen, D., 1977. Zur Fazies und Stratigraphie der jungpleistozänen Ablagerungen im Trauntal (mit quartärgeologischer Karte). Jahrbuch der Geologischen Bundesanstalt 120, 1-130.

van Husen, D., 2000. Geological processes during the Quaternary. Mitteilungen der Österreichischen Geologischen Gesellschaft 92, 135-156.

Welten M., 1982. Vegetationsgeschichtliche Untersuchungen in den westlichen Schweizer Alpen: Bern-Wallis. Denkschriften der Schweizerischen Naturforschenden Gesellschaft 95, 1-105.

Walker, M.J.C., Björck, S., Lowe, J.J., Cwynar, L.C., Johnsen, S., Knudsen, K.-L., Wohlfahrt, B., INTIMATE group., 1999. Isotopic 'events' in the GRIP ice core: a stratotype for the Late Pleistocene. Quaternary Science Reviews 18, 1143-1150. 


\title{
Die Bedeutung des Längsees in Kärnten für die Rekonstruktion der Klima- und Seenentwicklung am Ende der letzten Eiszeit
}

\author{
Schmidt R. ${ }^{1)}$, Drescher-Schneider R. ${ }^{2)}$, Huber K. ${ }^{1)}$, Weckstöm K. ${ }^{1)}$ \\ 1) Institut für Limnologie, Österreichische Akademie der Wissenschaften, Mondsee, \\ Österreich \\ 2) Schillingsdorferstraße 27 , Kainbach, Österreich
}

\section{Abstract}

Längsee in Carinthia as a key-site for the reconstruction of climate and lake development during the last glacial termination

Längsee is a small meromictic lake located in the southern pre-alpine lowland (Carinthia). For climatic reconstructions a multi-proxy approach was used (diatoms, ostracods, pollen, geochemistry, mineralogy, varves, tephra). By the use of a diatom-based transfer function for summer epilimnetic water temperatures (SEWT) three major sections were distinguished: (1) a warming period (average SEWT $15.8^{\circ} \mathrm{C}$, which is ca. $6^{\circ} \mathrm{C}$ lower than present), called Längsee oscillation, starting about 18 to $19 \mathrm{kyrs}$ before present (BP). It seems to correlate with the warmer event (GS-2b) indicated by the oxygen isotopes of the so-called Greenland Stadial 2; (2) a following climate deterioration, called Längsee cold period, which could correlate with the youngest cold event GS-2a of the Greenland Stadial GS-2 and the Heinrich I cold event from the Atlantic dated to 17.9 - $14.7 \mathrm{kyrs}$ BP. The SEWT difference of ca. $9^{\circ} \mathrm{C}$ between inferred and modern Längsee temperatures fits also well with the temperature decline assumed for the Gschnitz stadial of the Alps. Short-term fluctuations (SEWT range between 10.6 and $15.9^{\circ} \mathrm{C}$ ) indicate a tri-partition of this section (two colder phases are separated by a warmer fluctuation); (3) another warming period during the Lateglacial Interstadial (= Greenland Interstadial GI-1). The lower boundary of the initial birch-rich period was dated at Längsee to 14.3 kyrs BP using varves and a tephra layer (Neapolitan Yellow Tuff, NYT, from the Vesuvian area in Italy). Three minor climate fluctuations were observed (mainly by pollen) during this interstadial. The one likely related to the Gerzensee oscillation (Switzerland) or to the sub-stadial GI-1b in the sense of the Greenland isotope event-stratigraphy (both approximately dated to $13 \mathrm{kyrs} \mathrm{BP}$ ), showed a slight SEWT decline. The Younger Dryas cold period (= Greenland stadial GS-1) between ca. 12.7 - 11.5 kyrs BP appears to have been climatically heterogeneous, indicating a tri-partition in Längsee as well as in several other lake profiles of the Austrian Alps. Climate was also the main trigger of changes in nutrients and meromixis in Längsee. 


\section{Einleitung}

Der Längsee (548 m ü. M.) liegt im südöstlichen Alpenvorland Kärntens (Krappfeld nahe St. Veit a. d. Glan) im Randbereich der hochglazialen Moränen des würmzeitlichen Draugletschers (Lichtenberger 1959, Van Husen 1989). Als Würm wird die letzte der vier großen Vereisungen des Eiszeitalters (Pleistozän) in den Alpen bezeichnet. Während des Würm-Hoch oder Pleniglazials (Last Glacial Maximum $=$ LGM) stießen die Gletscher zwischen 23.000 bis 21.000 Jahren vor heute bis in das nördliche und südliche Alpenvorland vor.

Der Längsee weist heute eine sauerstoffarme bis -freie Tiefenzone auf, deren Ausdehnung saisonalen und klimabedingten Schwankungen unterworfen ist (z. B. Honsig-Erlenburg \& Schulz 1989, Sampl et al. 2001). Seen mit Teildurchmischung wurden von Findenegg $(1935,1937)$ als meromiktisch bezeichnet. Löffler (1973, 1975) erkannte, dass der Beginn der Meromixie in Kärntner Seen klimatische Ursachen hat und bis in das Spätglazial zurückreicht. Aufgrund dieser Eigenheiten war der Längsee der erste See Österreichs, der intensiv paläolimnologisch untersucht wurde (Frey 1955, 1956, Löffler 1973, 1975, Harmsworth 1984, Wunsam 1993 , Schmidt et al. 1998). Spätere Untersuchungen konzentrierten sich auf den Zeitraum zwischen dem LGM und dem Beginn des Holozäns vor ca. 11.500 Jahren, der auch als "last glacial termination" bezeichnet wird, bzw. gingen der Frage der komplexen

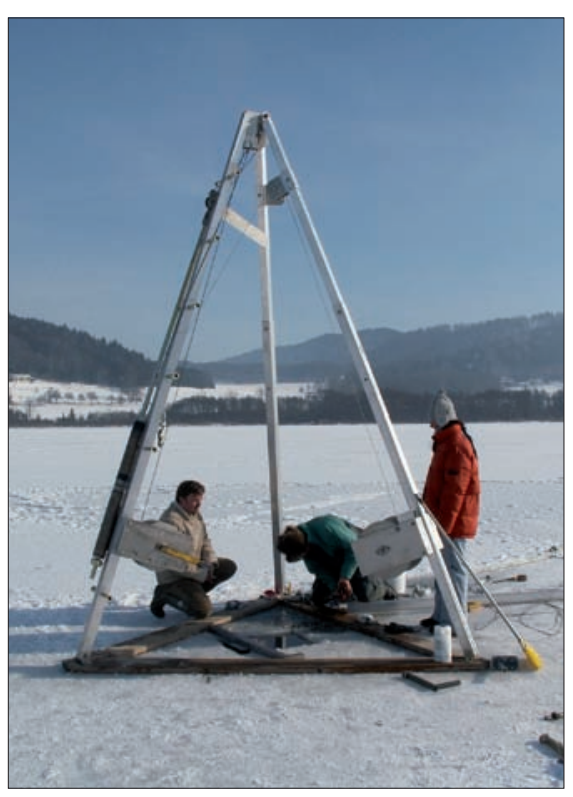
Zusammenhänge zwischen Klima, Meromixie und Nährstoffen in diesem Zeitabschnitt nach (Schmidt et al. 2002a, b). Jüngst wurden quantitative Methoden der Paläolimnologie für die Klimarekonstruktion eingesetzt (Huber et al., 2009). Die Paläolimnologie (VorzeitGewässerforschung) nützt die Ablagerungen (Sedimente) von Seen (Abb. 1) mit den darin enthaltenen Organismenresten für die Rekonstruktion von Klima- und Umweltvariablen.

Abb. 1. Bohrung des Sedimentkerns vom Eis des Längsees mit einem Rammkolbenlot (Firma UWITEC, Mondsee) (Foto K. Huber).

Fig. 1. Sediment coring from the frozen Längsee, using a piston sampler (UWITEC, Mondsee) (Photograph K. Huber). 


\section{Rekonstruktion der Klima- und Seenentwicklung mit Hilfe multidisziplinärer Methoden}

Der Wärmeanstieg nach dem Hochglazial führte zu einem raschen, wahrscheinlich nur einige Jahrhunderte dauernden Zerfall der alpinen Talgletscher. In dieser Eiszerfallslandschaft bildeten sich nach dem Abtauen sogenannter Toteisreste Seen, wie wahrscheinlich auch der Längsee. Während einer Warmphase nach dem LGM wurden im Längsee fossilführende (z.B. Pollen, Kiesel- und Goldalgen, Muschelkrebse) Sedimente abgelagert. Da im Längsee auf eine Warmphase wieder ein Kälterückschlag folgte, wurde diese Warmphase von Schmidt et al. (2001) als Längsee Oszillation bezeichnet. Zwei Radiokarbondaten $\left({ }^{14} \mathrm{C}\right)$ aus dem Bereich der Längsee Oszillation (Schmidt et al. 1998, Huber et al. 2009) ergaben ein Radiokarbonalter $\left({ }^{14} \mathrm{C}\right.$ ) von ca. 15.500 bzw. 15.700 vor heute (bezogen auf 1950), was einem geeichten (= kalibrierten) Alter von ungefähr 18.000 bis 19.000 Kalenderjahren entspricht. Eine Umrechnung der Radiokarbondaten in Kalenderjahre ist notwendig, da der Gehalt der Kohlenstoffisotopen $\left({ }^{13 / 14} \mathrm{C}\right)$ in der Atmosphäre Schwankungen unterworfen ist. Hinweise geben sogenannte ${ }^{14} \mathrm{C}$-Plateaus, d.h. Intervalle mit sehr geringer Veränderung oder sogar inversen ${ }^{14} \mathrm{C}$-Daten. Die Eichung (in Kalenderjahren) erfolgt u.a. mit ${ }^{14} \mathrm{C}$-datierten Hölzern und der Auszählung ihrer Jahresringe.

Die Pollenanalyse (Palynologie) bedient sich des in Ablagerungen (Seen, Moore) eingewehten Blütenstaubes (Pollen) von Bäumen, Sträuchern und Kräutern, um die Vegetation früherer Zeiten zu rekonstruieren. In der Längsee Schwankung kam es zu einer Verdichtung der Vegetationsdecke. Dies wird pollenanalytisch aus der Erhöhung der Typenzahl der Kräuter auf Kosten von Beifuß- (Artemisia) und Gänsefuß-reichen (Chenopodiaceae) Pollenspektren geschlossen, wie sie für Rohböden und steppenreiche Habitate unter dem kontinentalen Klima der ausklingenden Eiszeit charakteristisch sind (Abb. 2). Im Zuge dieser Warmphase dürfte es auch schon zu einer schwachen Ausbreitung kälteresistenter Gehölze wie Zwergweiden, Wacholder, Zwerg- und Stauchbirken und Föhren (Legföhre, Zirbe) gekommen sein, die entweder südlich der Alpen die letzte Eiszeit überdauert haben, oder von ihren südlichen eiszeitlichen Refugien wie zum Beispiel den Dinariden oder Italien rückgewandert sind (Avigliano et al. 2000, Schmidt et al. 2001). Die Zwergund Strauchbirke war in der späten Eiszeit in den Alpen weit verbreitet (siehe u.a. Drescher et al. 2007), beide weisen aber heute hier nur mehr Reliktstandorte auf. Das Vorkommen der Lärche im südlichen Alpenvorland während dieser Zeit ist 

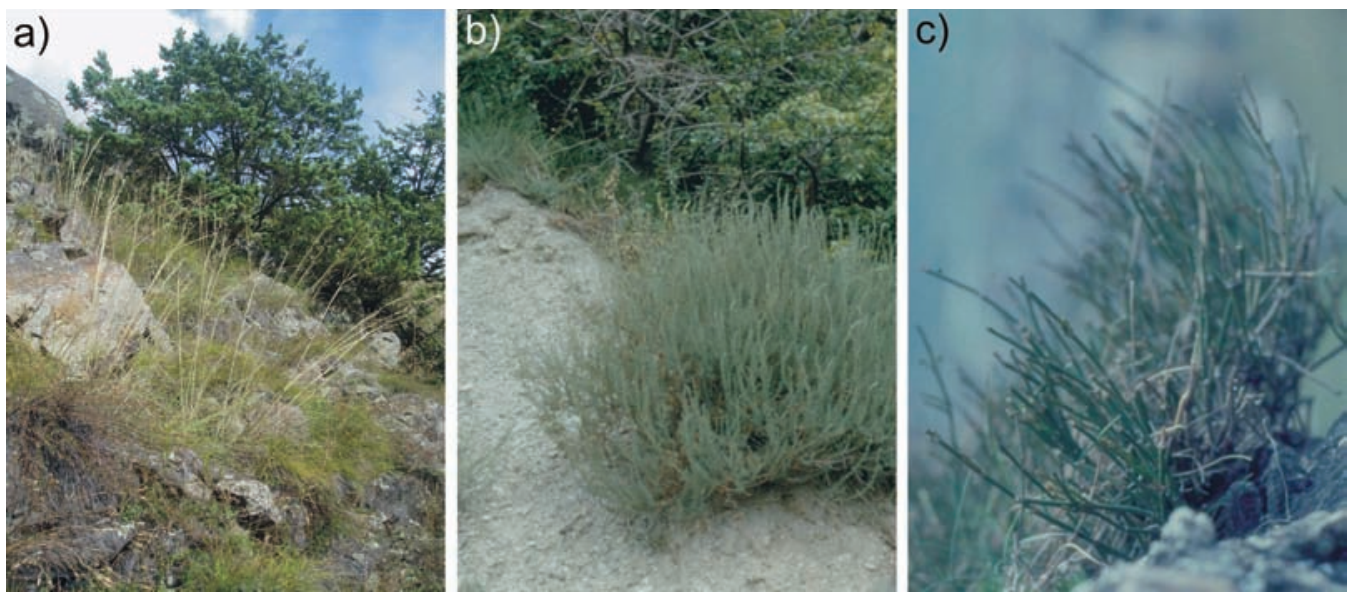

Abb. 2. Pflanzengesellschaften beutiger Steppen und Robböden, die, nach Pollenanalysen zu schließen, in der späten Eiszeit weit verbreitet waren: (a) Beifuß (Artemisia), Gräser- (u.a. Stipa) und Wacholder-reiche (Juniperus) Felsensteppe (Vinschgau); (b) Kochia prostrata (Gänsefußgewächse, Chenopodiaceae) auf Moränenanriss (Aostatal); (c) Ephedra distachya (Meeträubel), eine zu den Gymnospermen gehörende Pflanze mit Reliktstandorten in den Alpen (₹. B. Trento) (Fotos R. Schmidt).

Fig. 2. Association of plants from steppic habitats and initial soils, which, according to pollen analyses, were widespread during late glacial times: (a) Rock steppe rich in Artemisia, grasses (e.g. Stipa) and Juniperus (Vinschgau, South Tyrol, Italy); (b) Kochia prostrata (Chenopodiaceae) on glacial moraine (Val d'Aosta, Italy); (c) Ephedra distachya (Gymnospermae) on relict sites in the Alps (e.g. Trento, Italy) (Photographs R. Schmidt).

durch Funde von Baumstämmen bei Belluno, Norditalien, belegt (Casadore et al. 1976).

In Pollenprofilen des nördlichen Alpenvorlandes ist diese früher auch als Prä-Bølling (Schmidt 1975) bezeichnete Warmphase der südlichen Alpen (Föhrenvorstoß) nicht oder nur andeutungsweise erkennbar. Dies wird mit der größeren Distanz zu glazialen Gehölzrefugien und mit einem Süd-Nord Temperaturgefälle erklärt (siehe u.a. Fritz 1972, 1978). Im Gegensatz zu den Gehölzen konnten aquatische Organismen (wie die im Längsee nachgewiesenen Kiesel- und Goldalgen, sowie Muschelkrebse, siehe Abb. 3), mit kürzerer Reaktions- und Verbreitungszeit, schneller und intensiver auf das Wärmeangebot reagieren.

Kieselalgen (Diatomeen) aus Seeablagerungen stellen sensible Klimaindikatoren dar. Für die quantitative Rekonstruktion von Klima- und Umweltvariablen müssen diese Bio-Indikatoren jedoch geeicht (= kalibriert) werden. Für die Rekonstruktion der sommerlichen Wassertemperaturen aus den Kieselalgen des Längsees wurde ein Eichdatensatz aus 116 Seen der Alpen und Voralpen verwendet (Wunsam 1993, Wunsam et al. 1995, Schmidt et al. 2004). Mit Hilfe der in der Paläolimnologie gängigen Methode gewichteter Mittel (engl. "weighted averaging, WA“, siehe u.a. 
Birks 1998) wurde eine sogenannte Transferfunktion für die mittlere epilimnetische Wassertemperatur (SEW'T) erstellt und aus den fossilen Diatomeen des Längsees diese Variable berechnet (Huber et al., 2009). Für die Längsee Oszillation wurde ein mittlerer SEWT-Wert von $15,8{ }^{\circ} \mathrm{C}$ abgeleitet, was einer Temperaturdifferenz von ungefähr $6{ }^{\circ} \mathrm{C}$ gegenüber heute entspricht (siehe unten). Unsicherheiten in der Temperaturableitung ergeben sich aus dem modellspezifischen relativ hohen Standardfehler der Vorhersage von 1,8 ${ }^{\circ} \mathrm{C}$, sowie aus dem Vorkommen von Arten in der Späteiszeit (siehe u.a. Klee et al. 1993), die heute in den Seen des Eichdatensatzes nicht mehr vorkommen.

Luftemperatur und Niederschlag beeinflussen das Sauerstoff-Isotopenverhältnis $\left(\delta^{18} \mathrm{O}\right)$ in Gletschereis, Kalkablagerungen und in Kalkschalen von Wasserorganismen (z.B. Muschelkrebse; von Grafenstein et al. 1999, 2000). Damit stellen SauerstoffIsotopen empfindliche Klimasensoren dar. Die $\delta^{18} \mathrm{O}-\mathrm{Kurven}$ aus Bohrkernen grönländischer Eiskerne (GRIP und GISP, u.a. Grootes \& Stuiver 1993, Andersen et al. 2006, Rasmussen et al. 2006) werden vielfach für großräumige Klimavergleiche in der Nordhemisphäre verwendet. Zieht man die von Walker et al. (1999) erstellte Gliederung des späten Pleistozäns heran, die auf den Isotopenereignissen von GRIP basiert, so dürfte nach den Radiokarbondaten zu schließen die Längsee Oszillation dem wärmeren Intervall (GS-2b) des Grönland Stadials 2 (GS-2) entsprechen (siehe Abbildung 6). Die SEWT-Kurve dieser Erwärmung im Längsee weist größere kurzfristige Schwankungen auf mit einer Schwankungsbreite zwischen 11,6 und $18^{\circ} \mathrm{C}$. Die Temperaturmaxima reichen dabei an die nächste Warmphase, jene des
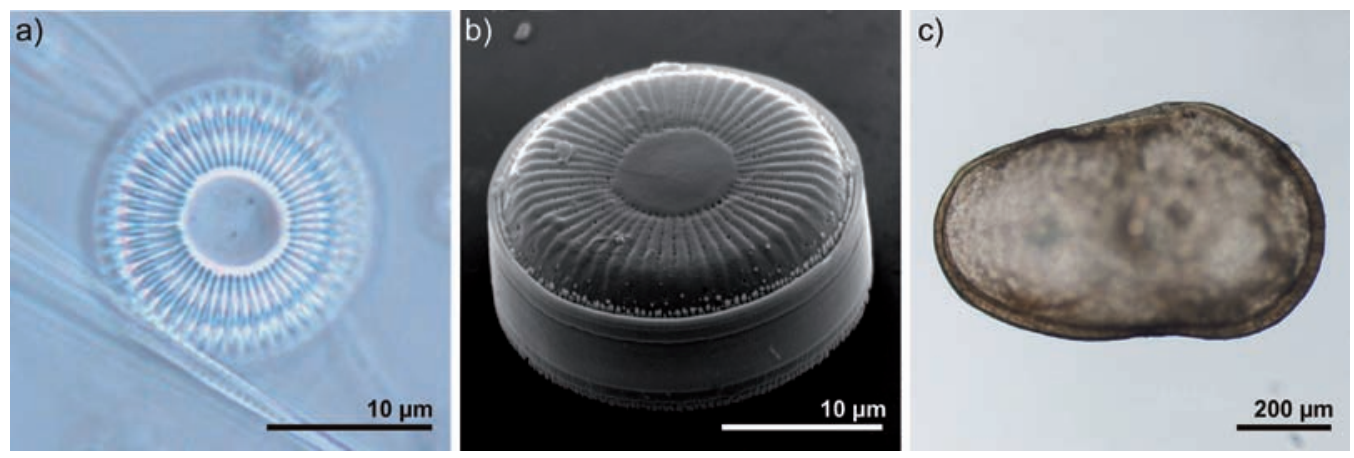

Abb. 3. a, b) Kieselalge Cyclotella plitvicensis im Licht- (a) und Elektronenmikroskop (b) (Fotos C. König/R. Klee) und c) Muschelkrebs Cytherissa lacustris (Foto M. Pichler/D.L. Danielopol) als Beispiele für Bewohner nährstoffarmer und relativ kübler Gewässer, die in der Späteiszeit weiter verbreitet waren als beute.

Fig. 3. a, b) The diatom Cyclotella plitvicensis in the light (a) and electron microscope (b) (Photographs C. König/R. Klee), and c) the ostracode Cytherissa lacustris (Foto M. Pichler/D.L. Danielopol) as examples of aquatic organisms from waters low in nutrients and temperature, which were wider distributed during late glacial times then at present. 
Spätglazialen Interstadials (= Grönland Interstadial 1, GI-1), heran (siehe unten). Die $\delta^{18} \mathrm{O}$-Werte von Kalkschalen eines Muschelkrebses (Cytherissa lacustris, Abb. 3) aus dem Sedimentkern des Längsees zeigten ebenfalls die klimatische Instabilität während der Erwärmungsphase der Längsee Oszillation (unveröff. Ergebnisse). Untersuchungen an rezenten Populationen zeigen, dass diese Art sehr sensibel auf Temperatur- und Umweltveränderungen reagiert (u.a. Danielopol \& Casale 1990).

Die folgende Absenkung der mittleren SEW'T um ca. $2,9^{\circ} \mathrm{C}$ gegenüber der Längsee Oszillation und des Minimalwerts auf $10,6{ }^{\circ} \mathrm{C}$, lässt auf eine deutliche Klimaverschlechterung schließen. Diese Längsee Kaltperiode wurde von Schmidt et al. (1998, 2001) mit der Pollenzone der Ältesten Dryas (Ia) korreliert. Die sogenannten Dryas-Zeiten, benannt nach der Silberwurz (Dryas ocotopetala), wurden ursprünglich in der Pollenanalyse als Vegetationszonen (Stratozonen) verwendet (Firbas 1954). Während des Kälterückschlags dominieren weiterhin kräuterreiche Pollenspektren. Nach den ${ }^{14}$ C-Daten könnte dieser Kälterückschlag dem Teilabschnitt GS-2a des Grönland Stadials 2 (GS-2) entsprechen. Kälterückschläge dieser Größenordnung werden auch als Stadiale bezeichnet. Der Kälterückschlag im Längsee könnte auch der als Heinrich Stadial 1 (H1) bezeichneten Kaltphase des Nordatlantik (Bond \& Lotti 1995) entsprechen, die zwischen 17.900 und 14.700 vor heute datiert wurde. Während dieser Kaltphase verschob sich die Grenze der nordpolaren Eisdrift nach Süden, was durch verfrachtetes Gestein in den Ablagerungen des Nordatlantiks angezeigt wird (,ice rafting events“) (Heinrich 1988). In den Alpen ist dieser stadiale Abschnitt durch eine neuerliche deutliche Gletschervorstoßphase nach dem Zerfall der alpinen Talgletscher gekennzeichnet. Der Gletschervorstoß des Gschnitz Stadials - benannt nach dem markanten Moränenwall am Ausgang des Gschnitztales in Tirol - wurde von Ivy-Ochs et al. (2006) der Kaltphase H1 zugeordnet (Kerschner et al. 2008). Die dafür geschätzte Absenkung der Lufttemperatur in diesem Stadial von 8,5 bis $10^{\circ} \mathrm{C}$ gegenüber heute, stimmt gut mit der Differenz der Wassertemperatur im Längsee zwischen $12,9^{\circ} \mathrm{C}\left(=\mathrm{SEW}_{\text {fossi }}\right)$ und $21,7^{\circ} \mathrm{C}$ (= SEW'T rezent , basierend auf Messdaten zwischen 2002 bis 2006, Kärntner Institut für Seenforschung) überein. Der Langzeittrend in den abgeleiteten Wassertemperaturen (Abb. 5) lässt eine angedeutete 3-Teilung erkennen. Zwei kältere Phasen sind durch eine Phase mit höheren Temperaturspitzen getrennt. Versucht man diesen paläolimnologischen Befund auf die Gletscherdynamik umzulegen, so könnten neben dem Gschnitz Stadial nach einer Rückzugsphase noch weitere, derzeit noch nicht genau einzuordnende Gletschervorstöße, untergebracht werden (Ivy-Ochs et al. 2008, Kerschner et al. 2008). 
Das folgende spätglaziale Interstadial (= Grönland Interstadial 1) dauerte von ca. 14.700 vor heute bis zum Beginn der Chronozone der Jüngeren Dryas, die mit ca. 12.650 vor heute angesetzt wird. Die Wiederbewaldung am Längsee wurde durch die Ausbreitung von Baumbirken eingeleitet, die relativ raschwüchsig sind und geringe Ansprüche an die Bodenbeschaffenheit stellen. Die Untergrenze dieser charakteristischen interstadialen Birkenausbreitung wurde im Längsee mit Hilfe einer vulkanischen Aschenlage (NYT = Gelber Neapolitanischer Tuff) und der Zählung von Jahreslagen (Warven) auf ca. 14.300 datiert (Schmidt et al. 2002 a). Der NYT konnte aufgrund seiner Gläser und des charakteristischen Chemismus einer Eruption des Vulkanismus um Neapel zugeordnet werden (Orsi et al. 1992). Er wurde im Lago Grande di Monticchio bei Neapel mit 14.120 Kalenderjahren datiert (Wulf et al. 2004) und wurde in den Alpen erstmals im Längsee nachgewiesen. Er ist damit älter als der in Mitteleuropa für das so genannte Allerød verwendete Leithorizont des Laacher See Tuffs (LST, ca. 12.000 vor heute, van den Bogaard et al. 1985). Diese aus einer vulkanischen Eruption des heutigen Laacher Maares in Deutschland stammende Asche wurde in Österreich bislang nur im Höllerer See nachgewiesen (Klee et al. 1993), einem kleinen Endmoränensee des würmzeitlichen Salzachgletschers nördlich von Salzburg.

Auf den birkenreichen Abschnitt folgt im Längsee ein föhrenreicher Abschnitt, an dem neben der Waldföhre (Pinus sylvestris) auch noch die Zirbe (Pinus cembra) beteiligt war (Abb. 4).

Abb. 4. Vergleichende Vegetationsbilder für das spätglaziale Interstadial des Längsees: (a) Tundrenvegetation mit Baumbirke (Betula alba) und Zwergbirke (Betula nana) als Unterwuchs im Uferbereich eines skandinavischen Sees; (b) Heutige Reliktföhrenwälder mit Waldföhre (Pinus sylvestris) und Verzahnung mit Zirbe (Pinus cembra) nabe der Waldgrenze (Südtirol) (Fotos R. Schmidt).

Fig. 4. Modern vegetation analogs from Scandinavia and the Alps for the late glacial interstadial of Längsee showing birch (Betula alba, $B$. nana at the shore of a Scandinavian lake) and pine-dominated (Pinus sylvestris, P. cembra) forest types close to the timber line in South Tyrol, Italy)(Photographs R. Schmidt).
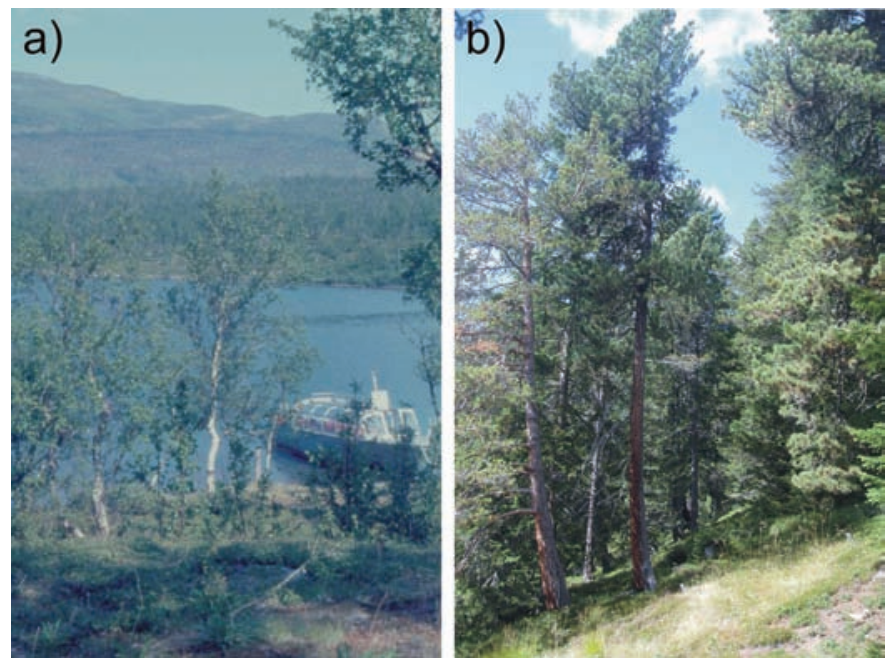
Innerhalb des spätglazialen Interstadials lassen Verschiebungen in den Pollenanteilen der Föhren, Einschwemmungen silikatischen Materials, sowie Veränderungen in der Zusammensetzung von Algenpigmenten (im Gegensatz zu den Kieselalgen bleiben von den meisten Algen nur die Pigmente in den Ablagerungen erhalten) auf drei kurzfristige Klimaschwankungen schließen. Die Klimaschwankung (Lg-FL1) am Übergang von der Birken- zur Föhrenphase liegt im Bereich des NYT und könnte damit der sogenannten Aegelsee Schwankung (ca. 13.800 v. h.), die jüngste (Lg-FL3) innerhalb der Föhrenphase der Gerzensee Schwankung (ca. 12.800 v. h.) der Schweiz (Lotter et al. 1992, Schwander et al. 2000) entsprechen. Von den drei Fluktuationen des Längsees war die Abkühlung während Lg-FL3 am deutlichsten im SEWTT ausgebildet. Dies stimmt sowohl mit dem signifikanten Pollenanstieg der Legföhre als Hinweis einer deutlichen Waldgrenzdepression unter möglicherweise schneereicheren Verhältnissen (Schmidt et al. 2002a), als auch mit dem Isotopensignal des GRIP Eiskerns (Walker et al. 1999) im Falle der Synchronität von Lg-FL3 mit GI-1b überein.

Gegen Ende der Längsee Kaltperiode begannen sich in der Tiefenzone des Längsees die Sauerstoffverhältnisse zu verschlechtern. Dies führte u.a. zu einem Ausfall sauerstoffbedürftiger Organismen in den Beckenablagerungen, wie am Beispiel der Muschelkrebse (Ostracoden) ersichtlich (Löffler 1973, 1975, Schmidt et al. 1998). Die Verschlechterung der Sauerstoffverhältnisse scheint ein gradueller Prozess gewesen zu sein. Mit der Klimaerwärmung des spätglazialen Interstadials trat Meromixie ein (Fehlen einer Vollzirkulation im See und damit verbundener sauerstoffarmer bis -freier Verhältnisse im Tiefenbereich). Dies belegen der Aufbau von Warven (= Jahresschichten) und die im Sediment erhaltenen Farbstoffe (Pigmente) spezifischer Bakterien und Algen (Okenon und Isorenieraten) (Schmidt et al. 2002b). Während Teildurchmischung im älteren Abschnitt des spätglazialen Interstadials noch wahrscheinlich war, dürfte Vollzirkulation des Sees auf die oben erwähnten Klimaschwankungen des spätglazialen Interstadials beschränkt gewesen sein. Die Meromixie erreichte im föhrenreichen Abschnitt ihren Höhepunkt. Dies kann u.a. aus der völligen Auflösung von Kalk (Kalzit) geschlossen werden. Als Ursache der Auflösung wurde eine durch eine stabile Schichtung des Sees bedingte $\mathrm{CO}_{2}$ Übersättigung des Tiefenwassers angenommen (Schmidt et al. 2002a, b). Dieser Abschnitt klimatisch induzierter strenger Meromixie dürfte mit dem vormals als Allerød bezeichneten Abschnitt synchron sein. Er dürfte niederschlags- (Schnee?) -ärmer als die vorangehende Birkenphase gewesen sein. Letztere deckt sich überwiegend mit dem vormals als Bølling bezeichneten Abschnitt. Beide, benannt nach Lokalitäten Dänemarks, wurden ursprünglich neben der Ältesten Dryas als 
Pollen- oder Stratozonen in Mitteleuropa verwendet, die einen bestimmten Vegetationstyp verkörpern. Die beschränkte Verwendbarkeit als Chronozonen wird jedoch u.a. daraus ersichtlich, dass die Birkenausbreitung am Längsee früher erfolgte (Schmidt et al. 2002a) als etwa nördlich der Alpen (Brauer et al. 1999a, Litt et al. 2001), sowie regionale Unterschiede auch noch durch andere Klimaeinflüsse wie zum Beispiel Trockenheit bedingt gewesen sein könnten (Drescher-Schneider et al. 2007).

Algen reagieren allgemein sehr sensibel auf Nährstoffveränderungen, vor allem auf Phosphor. Wunsam \& Schmidt (1995) erstellten ein auf Kieselalgen und Phosphormessungen in 86 Seen des Alpenraumes basierendes mathematisches Modell (Transferfunktion) für Gesamtphosphor (TP). Diese Transferfunktion wurde für die Rekonstruktion des Gesamtphosphors im spätglazialen Längsee verwendet. Im birkenreichen Abschnitt (Bølling s.l.) dürfte demnach das Nährstoffangebot durch zumindest temporär höhere Niederschläge und damit Nährstoffeintrag aus dem Einzugsgebiet sowie mögliche Teildurchmischung des Sees größer gewesen sein als im föhrenreichen Abschnitt (Allerød). In letzterem dürften verminderte Nährstoffeinträge aus dem Einzugsgebiet aufgrund des warm-trockenen Klimas, sowie strenge Meromixie und damit verbundene Phosphoranreicherung („Phosphorfalle“) im Tiefenbereich, zu nährstoffarmen (oligotrophen) Verhältnissen im oberflächennahen Stockwerk des Sees (= Epilimnion) geführt haben (Schmidt et al. $2002 \mathrm{a}, \mathrm{b})$.

Der Klimarückschlag der Jüngeren Dryas, der in der Grönländischen Terminologie (Walker et al. 1999) als Grönland Stadial 1 bezeichnet wird, ist eine weltweit verfolgbare markante klimatische Zäsur am Ende des Spätglazials. Die Chronozone der Jüngeren Dryas (YD) wird gewöhnlich zwischen 11.500 und 12.800 vor heute datiert (Brauer et al. 1999b, Litt et al. 2001). Dieser Klimarückschlag führte in den Alpen zu einer deutlichen Absenkung der Wald- und Schneegrenze und zu neuerlichen Gletschervorstößen (u.a. Kerschner et al. 2000). In Pollenprofilen aus den Alpen ist der Klimarückschlag der Jüngeren Dryas zumeist in einer Erhöhung des Nichtbaumpollen-Anteiles sichtbar. Im Längsee ist dieser dagegen nur geringfügig erhöht, was darauf hindeutet, dass dieser Klimarückschlag zu keiner größeren Lichtung der Wälder (hauptsächlich Waldföhre, Pinus sylvestris) in den Tieflagen am südöstlichen Alpenrand führte. Dagegen weist die Erhöhung in den Pollenanteilen von Zirbe und Legföhre in Form von Pollenfernflug sehr wohl auf Verschiebungen im Waldgrenzbereich hin. Zusammen mit Veränderungen in den sedimentologischen Kenngrößen zeichnet sich ein mehrphasiger Verlauf der Jüngeren Dryas ab. Die ältere Phase mit stärkeren Silikatanteilen (Quarz, Schichtsilikate) scheint kühl und 


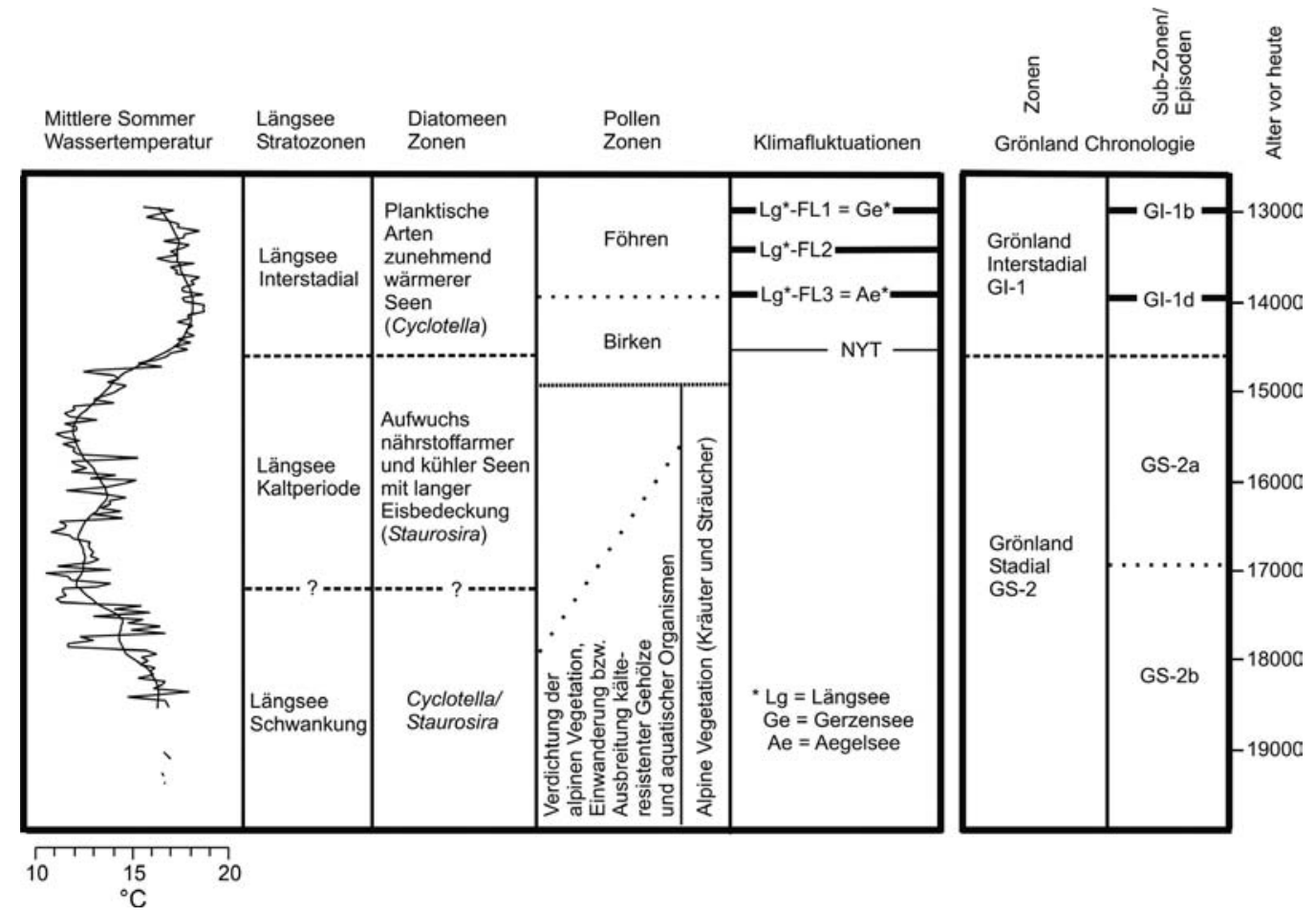

Abb. 5. Zonen-Gliederung des spät-eiszeitlichen Abschnittes zwischen ca. 19.000 und 13.000 vor heute, rekonstruiert mit Hilfe von Kieselalgen (Diatomeen), Pollen, einer Tufflage (NYT), Warvenzäblungen und Radiokarbondaten, in Sedimentkernen des Längsees (Schmidt et al. 1998, 2001, 2002, Huber et al., 2009). Korrelation der mittleren abgeleiteten Sommer-Wassertemperatur mit der GRIP-Isotopenstratigraphie Grönlands (nach Walker et al. 1999) und mit Klimafluktuationen des spätglazialen Interstadials im Alpenraum.

Fig. 5. Stratigraphy of Längsee in the time-window 19,000 to 13,000 years before present based on diatoms, pollen, a tephra-layer (NYT), varve counting and radiocarbon dating, of sediment cores. The correlation of the inferred mean summer water temperature with the GRIP isotope eventstratigraphy of Greenland (after Walker et al. 1999) and climate fluctuations within the late glacial interstadial in the Alps are added.

niederschlagsreich gewesen zu sein. Diese Klimaverhältnisse dürften im Längsee zu einer Durchmischung (temporäre Auflösung der Meromixie) geführt haben. Nach einem wahrscheinlich wärmeren Abschnitt, der auch als Interphase bezeichnet wurde (Schmidt 1984), folgt ein jüngerer Klimarückschlag. Diese in das Holozän überleitende Phase dürfte kontinentaler als der ältere Klimarückschlag gewesen sein. Diese Phasengliederung des Längsees deckt sich mit der Entwicklung in Seeprofilen aus den Nördlichen Kalkalpen (Salzkammergut) (Schmidt 1984) und mit dem Nachweis von zumeist zwei der Jüngeren Dryas zugerechneten Moränenstaffeln (Kerschner et al. 2000, 2008; Ivy-Ochs et al. 2008). 


\section{Schlussfolgerungen}

Das Zeitfenster zwischen ca. 19.000 und 12.000 vor heute zeigt im Längsees (Abb. 5) die Entwicklung von hoher klimatischer Instabilität (rasch aufeinanderfolgende Fluktuationen mit Temperaturextremen) zu zunehmender Stabilität. Zwei wärmere Perioden mit stufenweiser Erhöhung der mittleren Sommer-Wassertemperatur (Längsee Oszillation ca. $6{ }^{\circ} \mathrm{C}$ und spätglaziales Interstadial ca. $4{ }^{\circ} \mathrm{C}$ niedriger als heute) wurden von einer Kälteperiode (= Längsee Kaltperiode) unterbrochen, die wahrscheinlich überregional dem Grönland Stadial 2 bzw. der als Heinrich 1 bezeichneten Kaltphase des Nordatlantiks entspricht. Ähnlich wie die Klimaschwankung der Jüngeren Dryas (= Grönland Stadial 1), die den spätglazialen Abschnitt beendet, scheint auch dieser stadiale Abschnitt durch eine wärmere (trockenere?) Interphase geteilt. Das spätglaziale Interstadial (= Grönland Interstadial 1) zeigt im Längsee drei kurzfristige Kälteschwankungen, die sich auch in den Sauerstoff-Isotopensignalen von Eiskernen Grönlands und zwei Schwankungen in Sedimenten der Alpen wiederfinden, wobei die jüngste Fluktuation (= Gerzensee, Grönland GI-1b?) am deutlichsten ausgeprägt ist. Allgemein lässt das spätglaziale Interstadial am Längsee einen Trend von niederschlags- (Schnee?) reicheren (Bølling) zu warm-trockenen Verhältnissen (Allerød) erkennen.

\section{Danksagung}

Aufgrund des multidisziplinären Ansatzes und durch den Einbezug der Ergebnisse aus mehreren Veröffentlichungen ist vielen, hier nicht näher angeführten, Personen zu danken. Die jüngste Untersuchung von 2006 bis 2009 wurde/wird im Rahmen des Projektes P18595-B17 des Wissenschafts Fonds (FWF) durchgeführt. 


\section{Literatur}

Andersen K.K., Svensson A., Johnsen S.J., Rasmussen S.O., Bigler M., Röthlisberger R., Ruth U., Siggard-Andersen M-L., Steffensen J.P., Dahl-Jensen D., Vinther B.M., Clausen H.B. 2006. The Greenland Ice Core Chronology 2005, 15-42ka. Part 1: Constructing the time scale. Quat. Sci. Rev. 25: 3246-3257.

Avigliano R., Di Anastasio G., Improta S., Peresani M. \& Ravazzi C. 2000. A new Lateglacial to Early Holocene palaeobotanical and archaeological record in the Eastern Pre-Alps: the Palughetto basin (Cansiglio Plateau, Italy). - J. Quat. Scie. 15: 789-803.

Birks H.J.B. 1998. Numerical tools in palaeolimnology - progress, potentialities, and problems. J. Paleolimnol. 20: 307-332.

Bond G.C., Lotti R. 1995. Millenial-scale ice-rafting cycles in the North Atlantic during the last glaciation. Science 267: 1005.

Brauer A., Endres C., Negendank J.F.W. 1999a. Lateglacial calendar year chronology based on annually laminated sediments from Meerfelder Maar, Germany. Quat. Int. 61: 17-25.

Brauer A., Endres C. Günter C. Litt T., Stebich M., Negendank J.F.W. 1999b. High resolution sediment and vegetation responses to Younger Dryas climate change in varved lake sediments from Meerfelder Maar, Germany. Quat. Scie. Rev. 18: 321-329.

Casadoro G., Castiglioni G.B., Corona E., Massari F., Moretto M.G., Paganelli A., Terenziani F., Toniello V., 1976. Un deposito tardowürmiano con tronchi subfossili alle Fornaci di Revine (Treviso). Boll. Comit. Glac. It. 24: 22-63.

Danielopol D.L., Casale L., 1990. Long- and short-term perturbations of the Cytherissa lacustris populations in Mondsee: a paleolimnological perspective. Bulletin de l' Institut de Géologie du Bassin d'Aquitaine 47-48: 209-226.

Drescher-Schneider R., de Beaulieu J-L., Magny M., Walter-Simonnet A-V., Bossuet G., Millet L., Brugiapaglia E., Drescher A. 2007. Vegetation history, climate and human impact over the last 15,000 years at Lago dell'Accesa (Tuscany, Central Italy). Veget. Hist. Archaeobot. 16: 279-299.

Findenegg I. 1935. Limnologische Untersuchungen im Kärntner Seengebiet. Int. Rev. ges. Hydrobiol. 32: 369-423.

Findenegg I. 1937. Holomiktische und meromiktische Seen. Int. Rev. ges. Hydrobiol. 35: 586-610.

Firbas F. 1954. Synchronisierung der mitteleuropäischen Pollendiagramme. Danmarks geol. Undersoegelse 80: 12-21.

Frey D.G. 1955. Längsee: A history of meromixis. Mem. Ist. Ital. Idrobiol. Suppl. 8: 141-164.

Frey D.G. 1956. Die Entwicklungsgeschichte des Längsees in Kärnten. Carinthia II 146: 5-12.

Fritz A. 1972. Das Spätglazial in Kärnten. Ber. Deutsch. Bot. Ges. 85: 93-99.

Fritz A. 1978. Pollenstratigraphische Probleme des Würm- und des Postglazials in Kärnten. Carinthia II, 168: 189-206.

Grootes P.M., Stuiver M., White J.W.C., Johnsen S., Jouzel J. 1993. Comparison of oxygen isotope records from the GISP2 and GRIP Greenland Ice cores. Nature 366: 552-554.

Harmsworth R.V. 1984. Längsee: A geochemical history of meromixis. Hydrobiol. 108: 219-231.

Heinrich H. 1988. Origin and consequences of cyclic ice rafting in the northeast Atlantic ocean during the past 130,000 years. Quat. Res. 29: 142-152. 
Honsig-Erlenburg W., Schulz N. 1989. Der Längsee und seine fischereiliche Situation. Österreichs Fischerei 42: 245-253.

Huber K., Drescher-Schneider R., Weckström K., Schmidt R. 2009. Climate changes during the last glacial termination inferred from diatom-based temperatures and pollen in a sediment core from Längsee (Austria). J. Paleaolimnol, DOI 10.1007/s10933-009-9322-y.

Hughen K.A. et al. $2004 .{ }^{14} \mathrm{C}$ activity and global carbon cycle changes over the past 50,000 years. Science 290: 202-207.

Ivy-Ochs S., Kerschner H., Kubik P.W., Schlüchter C. 2006. Glacier response in the European Alps to Heinrich event 1 cooling: the Gschnitz stadial. J. Quat. Sci. 21: 115-130.

Ivy-Ochs S., Kerschner H., Reuther A., Preusser F., Heine K., Maisch M., Kubik P.W., Schlüchter C. 2008. Chronology of the last glacial cycle in the Northern European Alps. J. Quat. Sci. 23: 559573.

Kerschner H., Kaser G., Sailer R. 2000. Alpine Younger Dryas glaciers as palaeo-precipitation gauges. Ann. Glaciol. 31: 80-84.

Kerschner H., Ivy-Ochs S., Schlüchter C. 2008. Gletscher und Klima im Ostalpenraum zwischen 16.000 und 11.000 Jahren vor heute. Abhandl. Geol. Bundesanstalt 62: 165-168 (DEUQUA Wien 2008).

Klee R., Schmidt R., Müller J. 1993. Allerød diatom assemblages in prealpine hardwater lakes of Bavaria and Austria as preserved by the Laacher See eruption event. Limnol. 23: 131-143.

Lichtenberger E. 1959. Der Rückzug des Würmgletschers im mittleren Klagenfurter Becken und Krappfeld. Mitt. Österr. Geogr. Ges. 101: 37-62.

Litt T., Brauer A., Goslar T., Merkt J., Balaga K., Müller H., Ralska-Jasiewiczowa M., Stebich M., Negendank J.F.W. 2001. Correlation and sychronisation of Lateglacial continental sequences in northern and central Europe based on annually laminated lacustrine sediments. Quat. Sci. Rev. 20: 1233-1249.

Löffler H. 1973. Die Entwicklung der Meromixis in Klopeinersee und Längsee. Carinthia II 163: 331-377.

Löffler H. 1975. The onset of meromictic conditions in Alpine lakes. In: Suggate R.P., Cresswell M.M. (Eds), R. Soc. N.Z., Wellington, 211-214.

Lotter A.F., Eicher U., Siegenthaler U. 1992. Late-Glacial climate oscillations as recorded in Swiss lake sediments. J. Quat. Sci. 7: 187-204.

Orsi G., D’Antonio M., de Vita S., Gallo G. 1992. The Neapolitan Yellow Tuff, a large magnitude trachytic phreatoplinian eruption: eruptive dynamics, magma withd and caldera collapse. J. Vulcan. Geotherm. Res. 53: 275-287.

Rasmussen S.O., Andersen K.K., Svensson A.M.Steffensen J.P., Vinther B.M., Clausen H.B., SiggaardAndersen M-L., Johnsen S.J., Larsen L.B., Dahl-jensen D., Bigler M., Röthlisberger R., Fischer H., Goto-Azuma K., Hansson M.E., Ruth U. 2006. A new Greenland ice core chronology for the last glacial termination. J. Geophys. Res. 111: D06102.

Sampl H., Schulz L., Mairitsch M. 2001. Kärntner Seenbericht. Veröffentlichungen des Kärntner Institutes für Seenforschung 15: 58 pp.

Schmidt R. 1975. Pollenanlytische Untersuchungen zur spätglazialen bis mittelpostglazialen Vegetationsgeschichte im Raume Bozen. Linzer biol. Beitr. 7: 225-247. 


\section{Klimawandel in Österreich}

Schmidt R. 1984. Grundzüge der spät- und postglazialen Vegetations- und Klimageschichte des Salzkammergutes (Österreich) aufgrund palynologischer Untersuchungen von See- und Moorprofilen. Mitt. Komm. Quartärforsch. Österr. Akademie der Wissenschaften, 96pp.

Schmidt R., Wunsam S., Brosch U., Fott J., Lami A., Löffler H., Marchetto A., Müller H.-W., Pražákova M., Schwaighofer B. 1998. Late and post-glacial history of meromictic Längsee (Austria), in respect to climate change and anthropogenic impact. Aquat. Sci. 60: 56-88.

Schmidt R., Pugliese N., Müller J., Szeroczyńska K., Bogner D., Melis R., Kamenik C., Baric A., Danielopol D.L. 2001. Palaeoclimate, vegetation and coastal lake development, from upper Pleniglacial until early Holocene, in the northern Adriatic Valun Bay (Isle of Cres, Croatia). Il Quaternario 14: 61-78.

Schmidt R., van den Bogaard C., Merkt J., Müller J. 2002a. A new Lateglacial chronostratigraphic tephra marker for the south-eastern Alps: The Neapolitan Yellow Tuff (NYT) in Längsee (Austria) in the context of a regional biostratigraphy and palaeoclimate. Quat. Int. 88: 45-56.

Schmidt R., Psenner R., Müller J., Indinger P., Kamenik C. 2002b. Impact of late glacial climate variations on stratification and trophic state of the meromictic lake Längsee (Austria): validation of a conceptual model by multi proxy studies. J. Limnol. 61: 49-60.

Schmidt R., Kamenik C., Kaiblinger C., Hetzel M. 2004. Tracking Holocene environmental changes in an alpine lake sediment core: application of regional diatom calibration, geochemistry, and pollen. J. Paleolimnol. 32: 177-196.

Schwander J., Eicher U., Ammann B. 2000. Oxygen isotopes of lake marl at Gerzensee and Leysin (Switzerland), covering the Younger Dryas and two minor oscillations, and their correlation to the GRIP ice core. Palaeogeo., Palaeoclim., Palaeoecol. 159: 203-214.

van den Bogaard P., Schmincke H. 1985. Laacher See Tephra: a widespread isochronous Late Quaternary tephra layer in Central and Northern Europe. Geol. Soc. Am. Bull. 96: 1554-1571.

van Husen D. 1989. Die Entwicklung des Krappfeldes und seiner weiteren Umgebung im Pliozän und Pleistozän. Arbeitstagung der Geologischen Bundesanstalt 1989, 107-119.

von Grafenstein U., Erlenkeuser H., Brauer A., Jouzel J., Johnsen S.J. 1999. A mid-European decadal isotope-climate record from 15,500 to 5000 years B.P. Science 284: 1654-1657.

von Grafenstein U., Eicher U., Erlenkeuser H., Ruch P., Schwander J., Ammann B. 2000. Isotope signature of the Younger Dryas and two minor oscillations at Gerzensee (Switzerland): palaeoclimatic and palaeolimnologic interpretation based on bulk and biogenic carbonates. Palaeogeo., Palaeoclim., Palaeoecol. 159: 215-229.

Walker M.J.C., Björk S., Lowe J.J., Cwynar L.C., Johnsen S., Knudsen KL., Wohlfarth B. 1999. INTIMATE group. Isotopic «events» in the GRIP ice core : a stratotype for the Late Pleistocene. Quat. Sci. Rev. 18:1143-1150.

Wulf S., Kraml M., Brauer A., Keller J., Negendank J.F.M. 2004. Tephrochronology of the 100 ka lacustrine sediment record of Lago Grande di Monticchio (southern Italy). Quat. Int. 122: 7-30.

Wunsam S. 1993. Diatomeen (Bacillariophyceae) als Bioindikatoren in Alpenseen. Transferfunktionen zwischen Diatomeen und Umweltvariablen (ALPTROPH). Diss. Univ. Wien, 147 pp.

Wunsam S., Schmidt R., Klee R. 1995. Cyclotella-taxa (Bacillariophyceae) in lakes of the Alpine region and their relationship to environmental variables. Aquat. Scie. 57: 360-386.

Wunsam S., Schmidt R. 1995. A diatom-phosphorus transfer function for Alpine and pre-alpine lakes. Mem. Ist. Ital. Idrobiol. 53: 85-99. 


\title{
Alpine Dendrochronologie - Untersuchungen zur Kenntnis der holozänen Umwelt- und Klimaentwicklung
}

\author{
Kurt Nicolussi \\ Institut für Geographie, Universität Innsbruck, Österreich
}

Abstract

Alpine Dendrochronology - Research on Holocene Environmental and Climate Change

Dendrochronological research in the Alps takes place since the 1940s, but until recent years a Holocene tree-ring chronology based on Alpine samples has been missed. The new EasternAlpine Conifer Chronology (EACC, from 7109 B.C. to A.D. 2002) discussed here is with its length of more than 9000 years the second longest tree-ring chronology in the world. Based on the EACC, new results were obtained regarding Holocene glacier and tree-line variability in the Alps. These dendrochronological results indicate that on average the early and middle Holocene (until about 4000 years BP) was significantly warmer than the late Holocene. To date, dendroclimatological temperature reconstructions for the Alps cover the last some 1000 years. The reconstructed temperature evolution is in accordance with the known environmental evolution, particularly with the glacier history.

\section{Einleitung}

Möglichst präzise chronologische Angaben sind in der Paläoforschung in der Regel die einzige Möglichkeit, um an verschiedenen Orten beobachtete Ereignisse und Abläufe miteinander in Verbindung zu bringen, ein Prozessverständnis zu entwickeln und Kausalitäten zu bestimmen. Als Datierungsstandard für die letzten Jahrzehntausende dient hierbei die Radiokarbonmethode, die in den letzten Jahrzehnten eine deutliche Steigerung der Leistungsfähigkeit und Präzision erfahren hat. Allerdings bestehen aufgrund der Variabilität des ${ }^{14} \mathrm{C}$-Gehaltes in der Vergangenheit sowie messtechnisch bedingt Schranken bezüglich der Genauigkeit der zeitlichen Festlegung. Letzteres ist ein Aspekt, der auch für andere verwendete radiometrische Datierungsmethoden, wie der Uran-Thorium-Methode, gültig ist. Demgegenüber weist die Dendrochronologie mit dem Potential der jahrgenauen Bestimmung, sowohl relativ- als auch absolutchronologisch, ein höheres Potential auf. Einschränkungen sind vor allem aufgrund der Verfügbarkeit von für dendrochronologische Analysen geeignetem Material gegeben. Darüber hinaus gibt es im Alpenraum auch praktisch eine prinzipielle zeitliche Beschränkung auf das Holozän beziehungsweise das Spätglazial. 
Neben dem Einsatz der Dendrochronologie als Datierungsmethode, sei es im archäologisch/bauhistorischen Bereich oder in der Paläoumweltforschung, kann das Jahrringmaterial selbst auch analysiert und die erarbeiteten Daten für Rekonstruktionen und Analysen der Umwelt- und Klimaentwicklung verwendet werden. Die Datenerfassung beschränkt sich nicht nur auf die Erstellung von Serien und Chronologien der Gesamtjahrringbreite, dem am einfachsten zu erfassenden Jahrringparameter bei Holzgewächsen der mittleren und hohen Breiten, auch Früh- bzw. Spätholzbreiten sowie vor allem auch die Holzdichte sind analysierbar und verwendbar. Darüber hinaus kann das Holz selbst für die Bestimmung bestimmter chemischer Inhaltsstoffe aber auch für die Analyse von stabilen und instabilen Isotopen verwertet werden. Ein schon seit Jahrzehnten gepflegter Anwendungsbereich ist die Erstellung von jahrring-basierten Kalibrationskurven für die Radiokarbondatierungsmethode (z.B. Reimer et al. 2004).

Der Alpenraum stellt aufgrund der Höhenerstreckung aus dendrochronologischer Sicht ein komplexes Areal dar, in dem die das Jahrringwachstum beeinflussenden und limitierenden Faktoren durchaus unterschiedlich sind. Ist es in den Talräumen und bei entsprechendem Untergrund oftmals Trockenstress während der Vegetationszeit, der das Jahrringwachstum limitiert (z.B. Oberhuber und Kofler 2002), so ist demgegenüber die Sommertemperatur im Hochlagen- und vor allem im Waldgrenzbereich der ausschlaggebende Faktor. In mittleren Höhenbereichen und manchen Tallagen ist die Wachstumssteuerung nicht so eindeutig. Sowohl kalt/feuchte wie auch heiß/trockene Bedingungen während der Vegetationszeit können zu ähnlichen Wachstumsergebnissen führen (Becker 1991). Ein in Gebirgsräumen wie den Alpen oftmals angewandtes Konzept im Hinblick auf Klimarekonstruktionen ist daher die Bearbeitung von Hölzern von vergleichsweise extremen Standorten, wie der alpinen Waldgrenze, wo die Klimasteuerung des Wachstums eindeutig ist.

\section{Alpine Chronologie}

Die Waldvegetation der Alpen wird dominiert von Nadelbaumarten, was sich bei den bearbeiteten Holzarten manifestiert. Obwohl im Alpenraum bereits kurz nach der Einführung der Dendrochronologie in Europa durch Huber (1941) erste Arbeiten an den Nadelholzbaumarten Zirbe und Lärche (Pinus cembra L. bzw. Larix decidua Mill.) aus den Alpen durchgeführt wurden (Artmann 1949, Brehme 1951), fehlten lange Zeitreihen beziehungsweise Chronologien, die kalenderdatiert über die 
letzten rund 1000 Jahre hinausgingen. Zwar gab es bereits in den 1970er und 1980er Jahren vor allem in der Schweiz Bemühungen um den Aufbau einer holozänen Jahrringchronologie, wobei auch eine Reihe von so genannten „schwimmenden“, d.h. ${ }^{14} \mathrm{C}$-datierten Chronologien für die letzten knapp 8000 Jahre erarbeitet werden
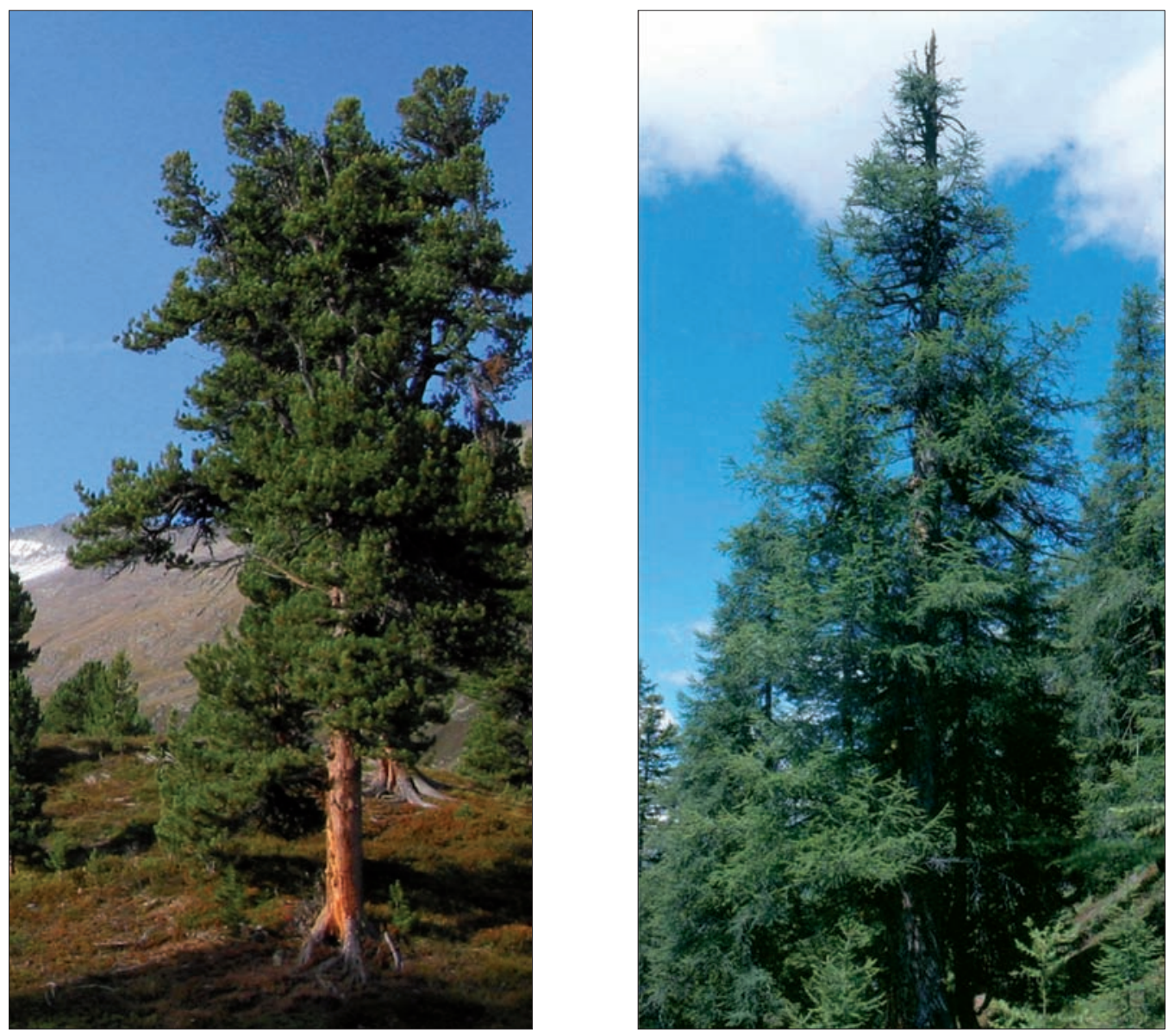

Abb. 1: Die wichtigsten Nadelbaumarten des Hocblagenbereiches der Zentralalpen: Zirbe (Pinus cembra L., links) und Lärche (Larix decidua Mill., rechts). Individuen beider Baumarten können in der Regel mehrere bundert Jahre, im Extremfall auch etwa 1000 Jahre alt werden. Die Jahrringentwicklung dieser Baumarten an Waldgrensstandorten wird wesentlich durch die sommerlichen Temperaturverbältnisse bestimmt beziebungsweise limitiert (Aufn. K. Nicolussi).

Fig. 1: The most important conifer species of the high altitude region of the Central Alps: stone pine (Pinus cembra L., left) and larch (Larix decidua Mill., right). In rare cases individuals of either species can reach lifespans of up to 1000 years. Tree-rings of these species growing at the tree line mainly reflect temperature regimes during summer (Photographs K. Nicolussi). 
konnten, eine Erstellung der angestrebten Holozän-Chronologie gelang jedoch bis zum Ende des 20. Jahrhunderts nicht (Giertz-Siebenlist 1977, Röthlisberger et al. 1980, Bircher 1982, Renner 1982, Schär und Schweingruber 1987, 1988). Erst in den letzten etwa 10 Jahren war es möglich im Zuge mehrerer Forschungsprojekt eine durchgehende Chronologie für die letzten rund 9100 Jahre, basierend vor allem auf Hölzern des mittleren Ostalpenraumes, zu erstellen (Nicolussi et al. 2004, Nicolussi et al., under review). Darüber hinaus wurden auch für das früheste Holozän bereits mehrhundertjährige, derzeit allerdings noch „schwimmende“ Jahrringchronologien erarbeitet (Abb. 1). An der 9100 Jahre langen Ostalpinen Nadelholz-Chronologie (Eastern Alpine Conifer Chronology, EACC), die auf 1432 subfossilen und subrezenten Holzproben vor allem der Baumarten Zirbe und Lärche beruht (Stand: 2007), waren auch mehrere „schwimmende“ Reihen und Chronologien aus dem ganzen Alpenraum synchronisierbar und damit kalender-datierbar (Abb. 1). Damit konnte sich ein alpenweites Netzwerk von holozänen Nadelholzchronologien etablieren, in dem durchwegs Jahrringserien aus dem Hoch- und Waldgrenzbereich der Alpen korreliert werden konnten, was auf eine übereinstimmende beziehungsweise ähnliche Steuerung des Jahrringwachstums in diesem Bereich zurückzuführen ist. Jahrringdatensätze aus tiefer gelegenen Alpenbereichen, die vor allem aus archäologischen Fundkomplexen stammen, lassen sich hingegen in der Regel nicht oder kaum mit diesem Hochlagendatensatz synchronisieren.

\section{Potenzial und Ergebnisse}

Der wesentliche Anwendungsbereich der Ostalpinen Nadelholz-Chronologie (EACC) ist die Paläoumwelt- und Klimaforschung. Wesentliche Ergebnisse waren für die Kenntnis der holozänen Gletscherentwicklung in den Alpen erarbeitbar. Eine Reihe von Vorstößen des Gepatschferners, Tirol, während der letzten 4000 Jahre konnten aufgrund der Datierung von überfahrenen Bäumen präzise datiert werden (Nicolussi und Patzelt 2001). Auch die weitreichenden Vorstöße des Großen Aletschgletschers Wallis in den beiden Jahrtausenden vor und nach Christi Geburt, die so genannten Göschenen I und II Vorstöße, waren durch die Synchronisation der Jahrringdatensätze von überschütteten Hölzern an der EACC kalenderdatierbar (Holzhauser et al. 2005).

Während Hölzer aus alpinen Gletschervorfeldern aus dem Zeitraum der letzten rund 4000 Jahre in der Regel weit reichende Vorstöße der Alpengletscher dokumen- 
tieren und datierbar machen, hat der aktuelle markante Gletscherrückgang in den letzten Jahren zur Freilegung von Hölzern unmittelbar an manchen Gletscherenden geführt. Diese Hölzer belegen in der Regel kleinere Gletscherstände als gegenwärtig und sind darüber hinaus nahezu ausnahmslos älter als 4000 Jahre (Nicolussi et al. 2008). Beispielsweise sind am Tschierva Gletscher, Engadin, bisher rund 50 solche Hölzer vom Gletscher freigegeben worden, die mit wenigen Ausnahmen dendrochronologisch datierbar waren und überwiegend in den Zeitraum zwischen rund 5500 und 4650 v. Chr. fallen (Jörin et al. 2008). Solche Holzfunde mit Daten aus dem frühen und mittleren Holozän sind auch von anderen Gletschern bekannt (Hormes et al. 2001, Jörin et al. 2006, Nicolussi et al. 2008). Besonders bemerkenswert ist die teilweise lange Lebenszeit - bis über 800 Jahre - der erfassten Baumreste, die folglich lange andauernde Rückzugsphasen der alpinen Gletscher jenseits der heutigen

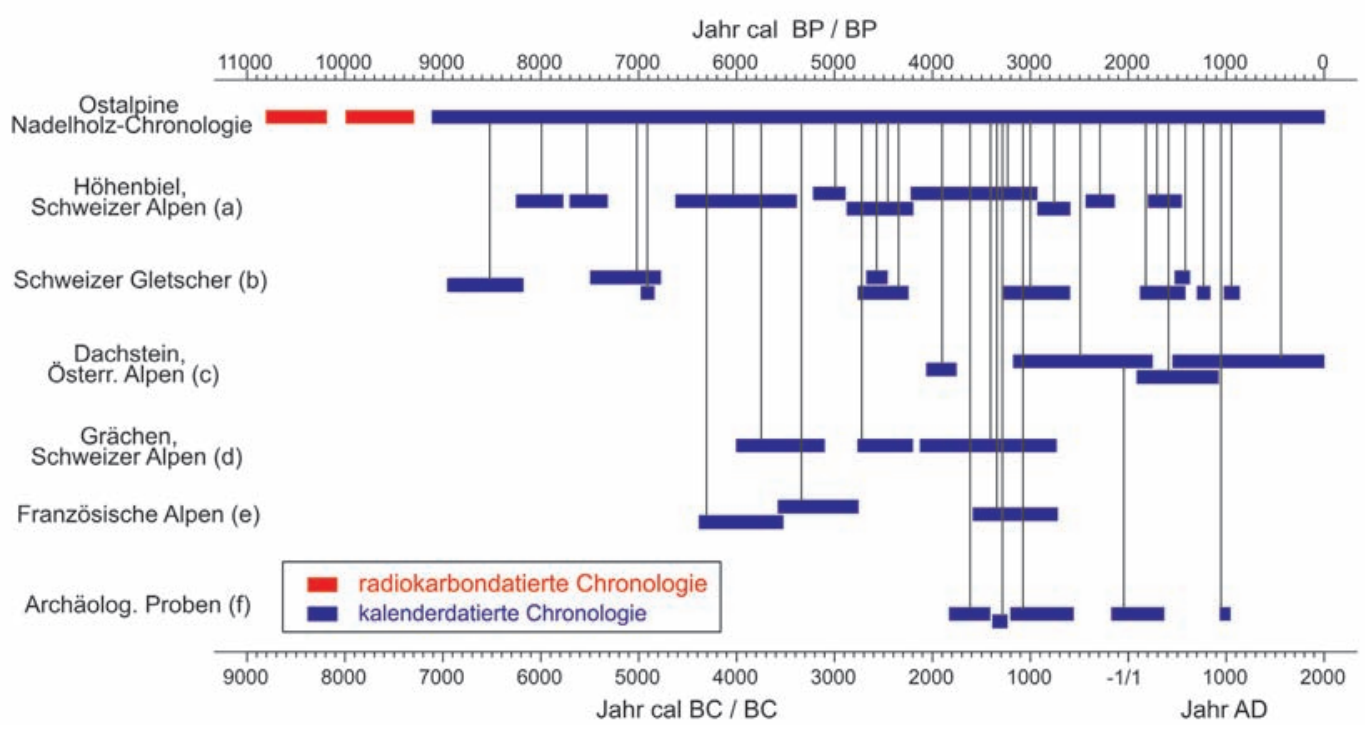

Abb. 2: Das alpine Netzwerk der Nadelholz-Jahrringserien und Chronologien. Die Kalenderdatierung von ursprünglich schwimmenden Chronologien basiert auf deren Synchronisation mit der Ostalpinen NadelholzChronologie. Referenzen: a) Renner 1982; Bircher 1982; b) Renner 1982; Holzhauser 1997, unpubl.; Jörin et al. 2006; Jörin et al. 2008; Nicolussi et al. unpubl.; c) Grabner und Gindl 2000; Grabner et al. 2001; d) Schär und Schweingruber 1987, 1988; e) Edouard et al. 2002; f) Seifert 2000; Reitmaier und Nicolussi 2002; Pichler et al. im Druck; Nicolussi et al. unpubl.

Fig. 2: The Alpine network of conifer tree-ring series and chronologies. Calendar dating of previously floating chronologies and single tree-ring series from whole Alpine region is based on crossdating with the Eastern-Alpine Conifer Chronology. Sources: a) Renner 1982; Bircher 1982; b) Renner 1982; Holzhauser 1997, unpubl.; Jörin et al. 2006; Jörin et al. 2008; Nicolussi et al. unpubl.; c) Grabner und Gindl 2000; Grabner et al. 2001; d) Schär und Schweingruber 1987, 1988; e) Edouard et al. 2002; f) Seifert 2000; Reitmaier und Nicolussi 2002; Pichler et al. in print; Nicolussi et al. unpubl. 
Gletschergrenzen für das frühe und mittlere Holozän belegen. Eine geringere Ausdehnung als gegenwärtig dürfte der Normalsituation der Alpengletscher im frühen und mittleren Holozän entsprechen. Umgekehrt sind nach der Verteilung dieser Holzfunde aus verschiedenen Gletschervorfeldern der österreichischen und schweizerischen Alpen zeitlich ausgedehntere Vorstoßphasen im frühen und mittleren Holozän derzeit nur für die Zeiträume ca. 6100 bis 5700, 4700 bis 4200 und 3550 bis 3250 v. Chr. ableitbar.

Eine Reihe von Hölzern, die für den Aufbau der Ostalpinen Nadelholz-Chronologie (EACC) analysiert wurden, stammt von Lokalitäten der Zentralalpen, die

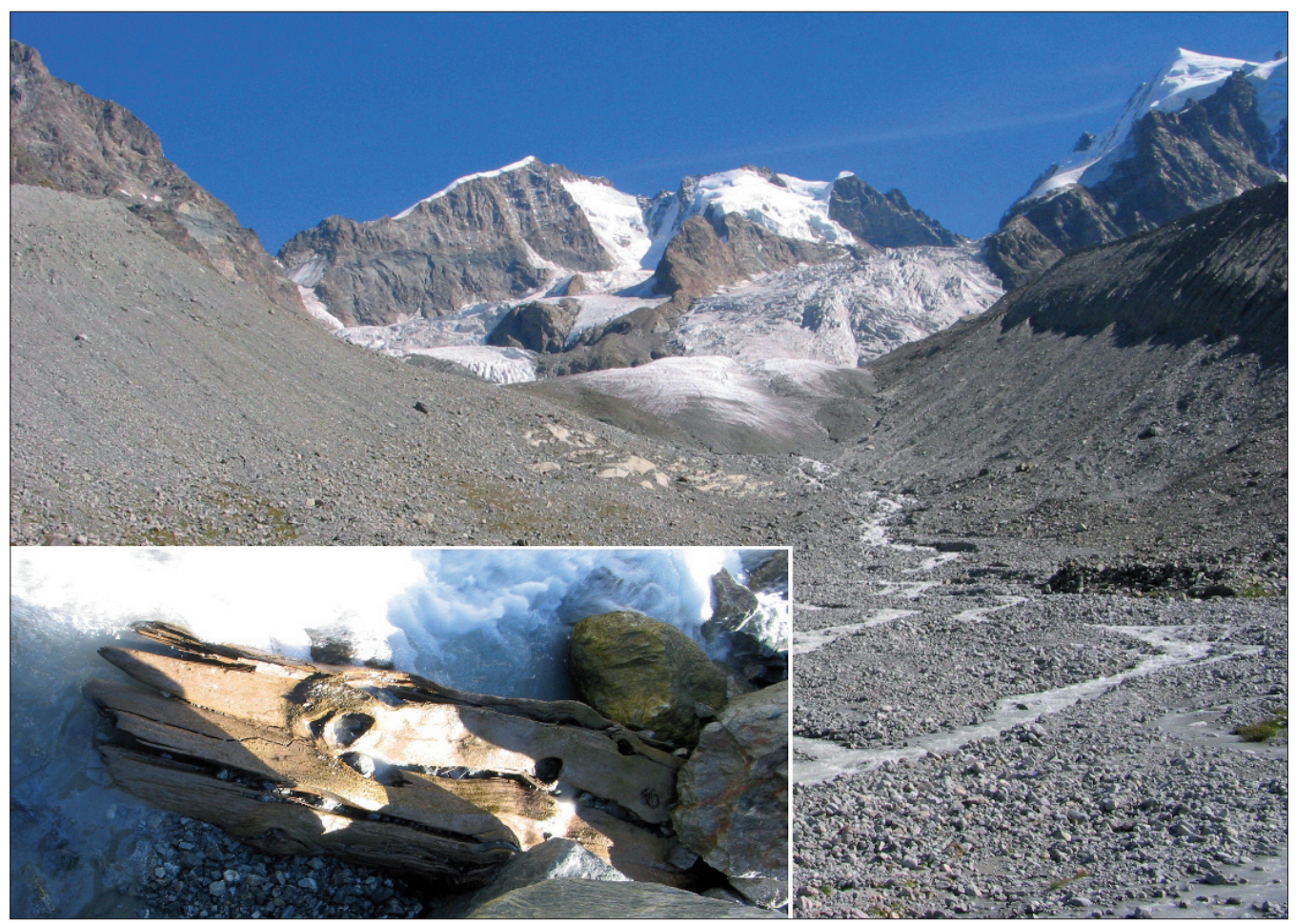

Abb. 3: Der Tschierva Gletscher in der Bernina Gruppe, Engadin. An diesem Gletscher waren in den letzten Jahren, fallweise direkt am zurück schmelzenden Eisrand, Baumreste von Zirben und Lärchen mit bis zu etwa 600 Jahrringen auffindbar. Diese Hölzer datieren mehrheitlich in den Zeitraum zwischen rund 5500 und 4650 v. Chr. (Jörin et al. 2008) und dokumentieren eine lange andauernde Gletscherrïck zugsphase (Aufn. K. Nicolussi, 2004/2007).

Fig. 3: The Tschierva glacier in the Bernina group, Engadin. In the past years several stone pine and larch trunks with up to 600 tree rings were found in the forefield of this glacier, partly directly at the melting ice margins. Most of these wooden remains date back to 5500 - 4650 B.C. (Jörin et al. 2005) and prove a long lasting glacier retreat period (Photographs K. Nicolussi, 2004/2007). 


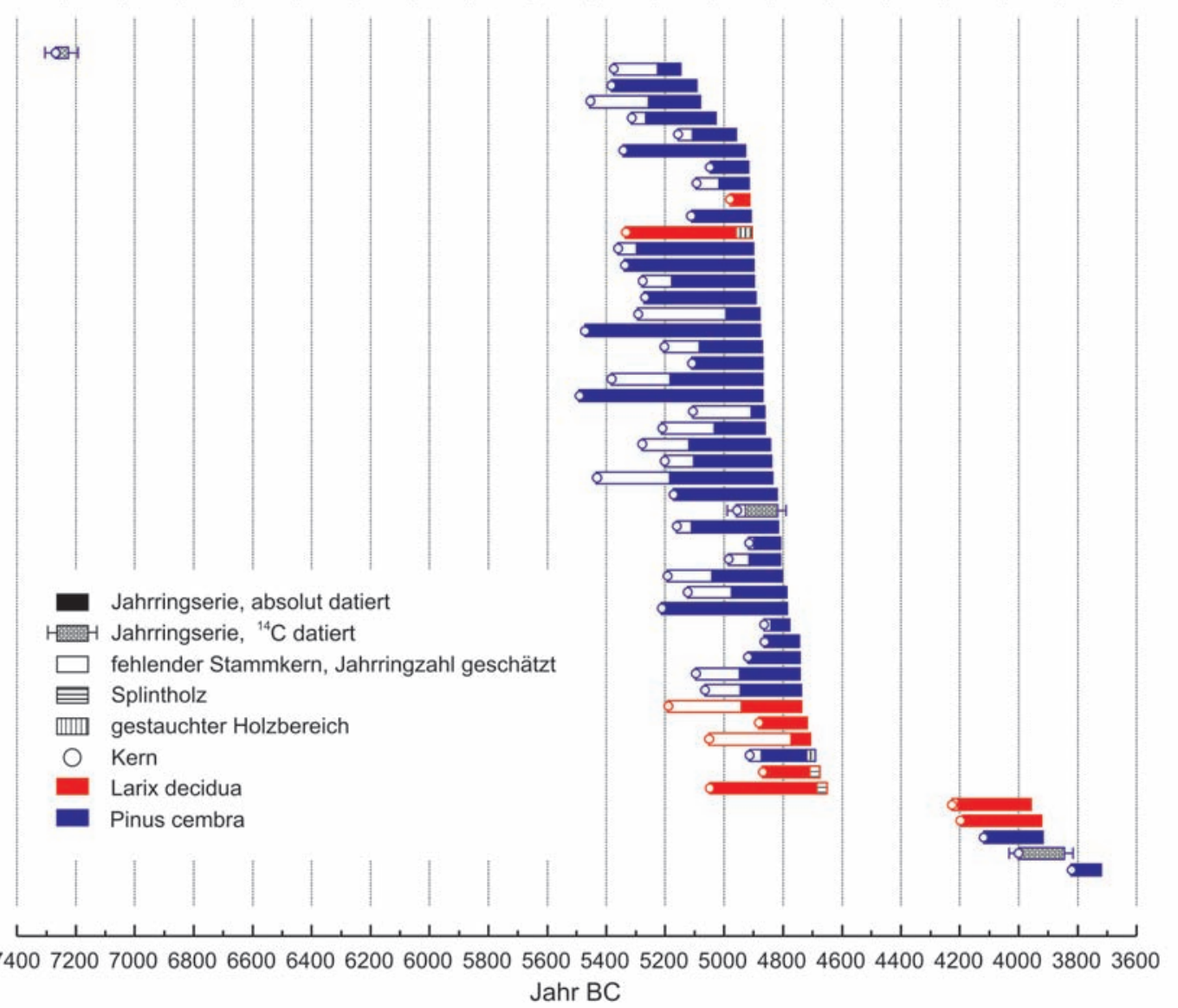

Abb. 4: Die zeitliche Verteilung von Baumresten, gefunden vor dem aktuellen Zungenende des Tschierva Gletschers, Engadin. Die Länge der farbigen Balken zeigt die zeitliche Erstreckung der an den Proben erarbeiteten Jabrringserie an, bei feblendem Stammkern wurde die Zabl der Jahrringe bis zum Kern geschätz̨t (Jörin et al. 2008, ergänఇt).

Fig. 4: Temporal distribution of tree remains from the Tschierva glacier, Engadin, found in front of the current tongue. The coloured bars indicate the length of the tree ring series established for each sample. Numbers of tree rings are estimated for a possibly missing inner part of a sample (Jörin et al. 2008, with supplemented data).

über der gegenwärtigen natürlichen Wald- beziehungsweise Baumgrenze liegen. Teilweise handelt es sich um mehrhundertjährige Baumreste, in deren Umgebung unter den Klimabedingungen des späten 20. und frühen 21. Jahrhunderts gerade Krüppelwuchs, jedoch kein hochstämmiger Baumwuchs möglich ist. Dass die alpine Baumgrenze, eine Temperaturmangelgrenze (Tranquillini 1979), auf die Klimaän- 


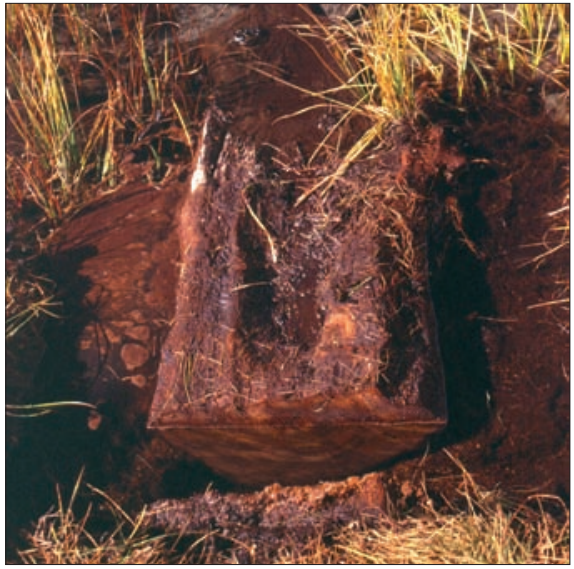

Abb. 5: Der Baum GDM-1 (2295 m SH, Jahrringserie 6483-6150 v. Chr.) starb während des 8.2k. Ereignisses ab. Der Fundort dieses Stammes liegt etwa $100 \mathrm{~m}$ böher als die Position der Baumgrenze um 1980 n. Chr. (Nicolussi et al. 2005) (Aufn. K. Nicolussi, 1994).

Fig. 5: The tree GDM-1 (2295 m a.s.l., tree ring series from 6483 to $6150 \mathrm{BC}$ ) died during the $8.2 \mathrm{k}$ event. The location of this log is about $100 \mathrm{~m}$ above the tree line of A.D. 1980 (Photograph K. Nicolussi, 1994).

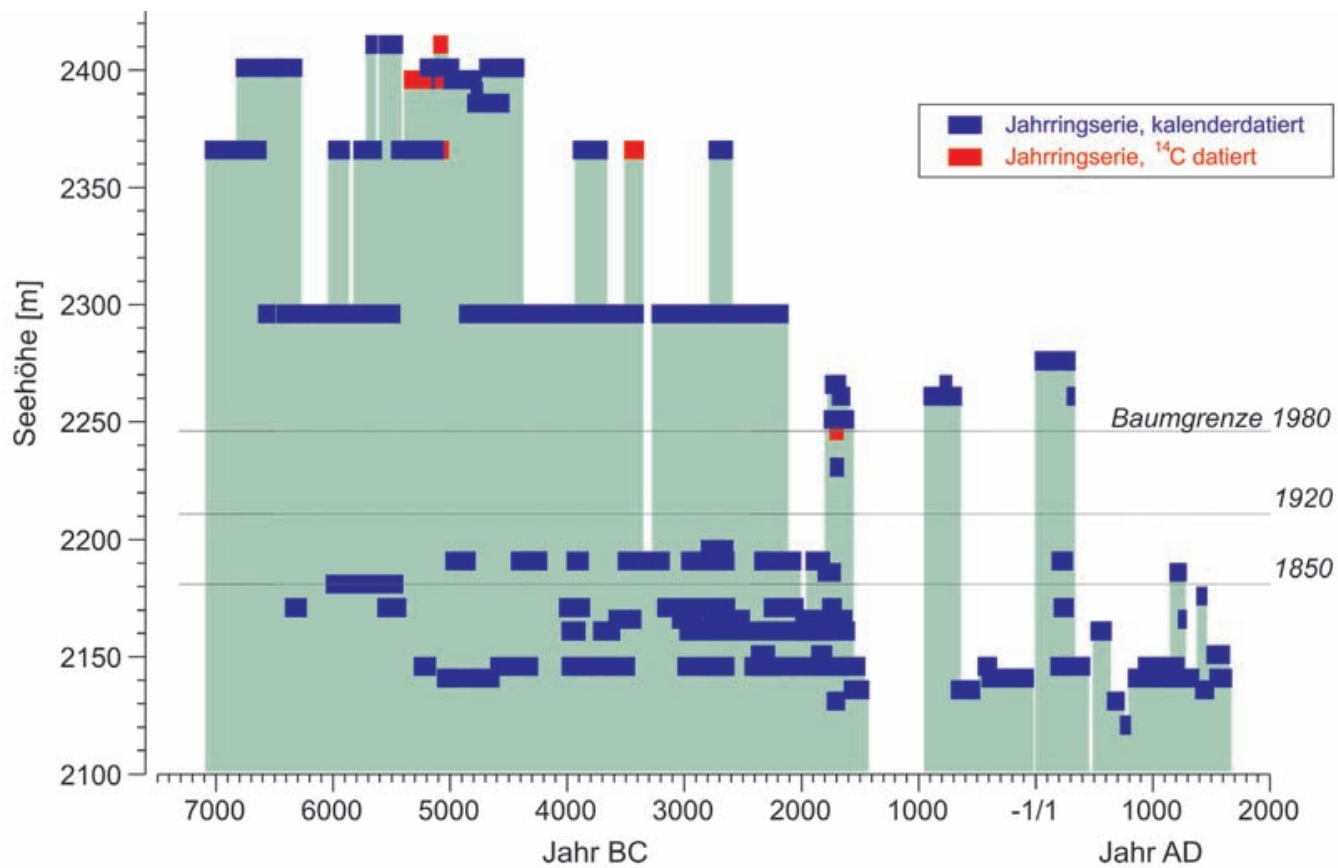

Abb. 6: Die höhenmäßige Verteilung von dendrochronologisch bzw. ${ }^{14} \mathrm{C}$-datierten Holzproben aus dem inneren Kaunertal, Westtirol, für den Zeitraum der letzten 9000 Jahre. Die Balken belegen jeweils einzelne Proben bzw. Gruppen von Proben mit gleicher Standorthöhe, die Balkenlänge zeigt die zeitliche Erstreckung der Jabrringserie einer Einzelprobe bzw. einer Standortmittelkurve an (Nicolussi et al. 2005, ergänzt).

Fig. 6: Altitudinal distribution of sub-fossil logs in the inner Kaunertal, western Tyrol. Age determination is based on dendrochronological or $14 \mathrm{C}$ analyses. The results scatter over the past 9000 years. Each bar represents a single sample or a group of samples found at the same altitude. The length of each bar indicates the length of the tree-ring series of each sample (Nicolussi et al. 2005, supplemented). 
derungen der letzten rund 150 Jahre durchaus mit einer Höherverlagerung reagiert, zeigen dendrochronologische Untersuchungen an rezenten Bäumen im Waldgrenzökoton der Zentralalpen (z.B. Nicolussi et al. 2005). Allerdings erreicht die heutige potenzielle Baumgrenzposition die holozänen Höchstlagen bisher nicht. Aus einer Zusammenstellung der Daten für das innere Kaunertal lässt sich ein holozäner Schwankungsbereich der Baumgrenze von zumindest 230 Höhenmetern ableiten. Abbildung 6 zeigt deutlich, das die hochgelegenen Baumrestfunde durchwegs in den Zeitraum vor rund 4000 Jahren datieren, was einerseits klimatisch bedingt sein dürfte, allerdings teilweise auch anthropogen beeinflusst sein könnte. Gerade die Lücken in der Belegung mit subfossilen Holzfunden, z.B. um 1200 v. Chr., dürften auf Rodungs- und Weidemaßnahmen durch den Menschen zurückzuführen sein. Die Kulmination an hochgelegenen Hölzern datiert in den Zeitraum um 5000 v. Chr. und damit zeitgleich mit der am Tschierva Gletscher nachgewiesenen langen Gletscherrückzugsphase (Abb. 4 und 6).

\section{Diskussion und Schlussfolgerungen}

Die Ergebnisse der neu analysierten und nun kalenderdatierten Hölzer haben dazu beigetragen, die Vorstellung von der holozänen Umwelt- und Klimaentwicklung im Alpenraum zu modifizieren. Lange wurde die seit gut 11.000 Jahren andauernde Nacheiszeit als eine Periode betrachtet, während der die klimatischen Verhältnisse um einen weitgehend gleich bleibenden Mittelwert vergleichsweise gering pendelten (z.B. Patzelt 1977, Haas et al. 1998). Der langfristige Temperaturschwankungsbereich wurde auf nur rund $1.3^{\circ} \mathrm{C}$ geschätzt. Die neuen Ergebnisse legen jedoch nahe, die Nacheiszeit grob in zwei Abschnitte zu unterteilen: das frühe und mittlere Holozän ist geprägt durch überwiegend weit zurückgezogene Gletscherzungen und eine Baum- und Waldgrenze, die allgemein über dem Niveau der letzten Jahre anzusetzen ist, andererseits ist für das folgende späte Holozän, ab etwa 4000 vor heute, eine durchschnittlich größere Gletscherausdehnung mit weit reichenden Vorstößen beziehungsweise wiederholten Hochständen und einer generell tieferen Baum- bzw. Waldgrenzhöhe anzunehmen (Abb. 7). Der Höhepunkt dieser Entwicklung ist die so genannte Kleine Eiszeit in der zweiten Hälfte des vergangenen Jahrtausends.

Der Wechsel von einem höheren zu einem niedrigeren Schwankungsniveau im Alpenraum um 4000 vor heute, wie er sich sowohl bei den Waldgrenz- als auch bei den Gletscherbefunden abzeichnet, erfolgte synchron mit anderen markanten, eben- 


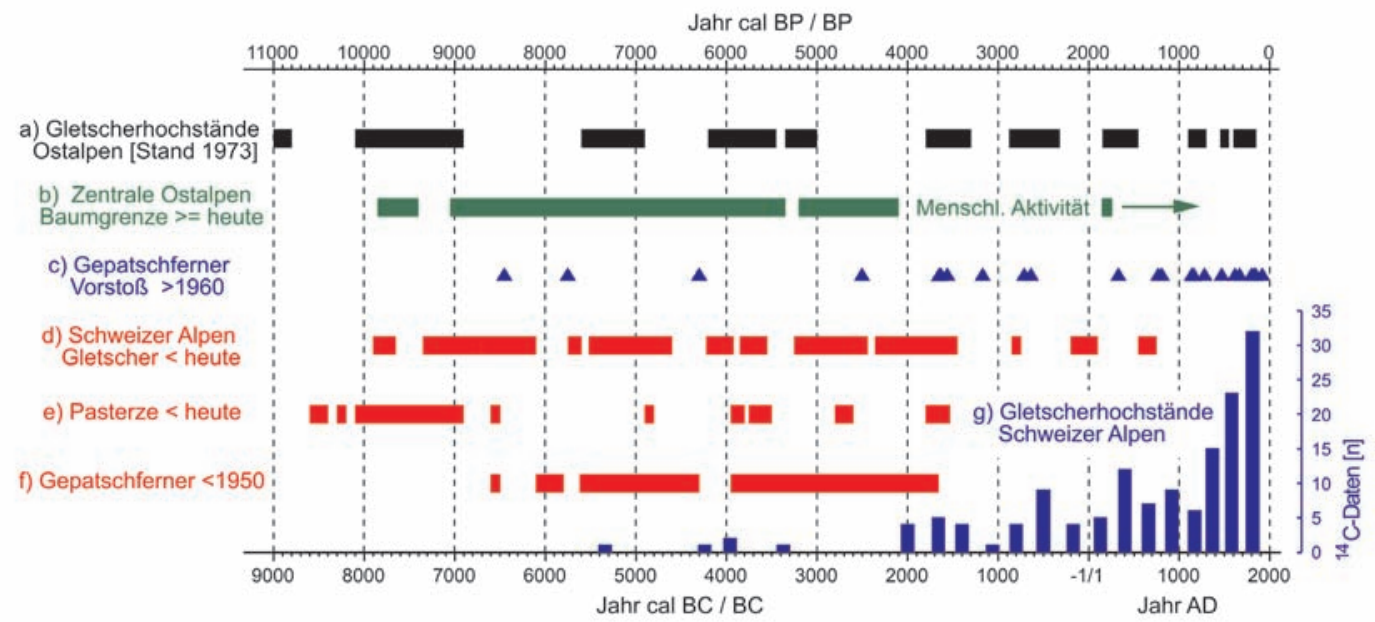

Abb. 7. Gletscher-und Baumgrenzentwicklung in der Nacheiszeit. Neue, oftmals dendrochronologisch basierte Ergebnisse zeigen überwiegend günstige Verbältnisse mit hoch gelegener Baumgrenze und meist weit zurück geschmolzenen Gletscherenden für das frühe und mittlere Holozän an. a) Vorstellung der Gletscherentwicklung in den Ostalpen, Stand 1973 (Patzelt und Bortenschlager 1973), b) dendrochronologische Belege für eine Baumgrenze in den Ostalpen höher als die heutige (Nicolussi et al. 2005, ergänఇt), c) Vorstöße des Gepatschferner über eine Ausdehnung wie um 1960 AD binaus (Nicolussi und Patzelt 2001), d) Gletscherausdehnung in den Schweizer Alpen kleiner als beute (Jörin et al. 2006; ergänæt mit neuen dendrochronologischen Belegen), e) und f) Ausdebnung der Pasterze kleiner als beute bzw. des Gepatschferners kleiner als 1950 AD (Nicolussi und Patzelt 2001) sowie g) Gletscherbochstände in den Schweizer Alpen nach ${ }^{14} \mathrm{C}$-Daten (Furrer et al. 1987).

Fig. 7: Holocene glacier and tree line evolution. New, often dendrochronologically based results indicate predominantly favourable climatic conditions with high tree line positions and usually small glacier extents for the early and mid-Holocene. a) Conception of the glacier evolution in the eastern Alps, state 1973 (Patzelt and Bortenschlager 1973), b) dendrochrono $\neg$ logical evidences for elevated tree lines compared to present (Nicolussi et al. 2005, supplemented), c) Holocene glacier advances of the Gepatschferner beyond the limits of A.D. 1960 (Nicolussi and Patzelt 2001), d) glacier extent in the Swiss Alps smaller than today (Jörin et al. 2006; new dendrochronological data supplemented), e) and f) extent of the glacier Pasterze smaller than "today" and of the Gepatschferner smaller than A.D. 1950, respectively (Nicolussi und Patzelt 2001), and g) Holocene glacier maxima in the Swiss Alps based on ${ }^{14} \mathrm{C}$ dating (Furrer et al. 1987).

falls klimatisch induzierten nordhemispärischen Umweltveränderungen. Um 5000 bis 4000 Jahre v.h. kommt es innerhalb einiger hundert Jahre zur Austrocknung der Sahara (Kröpelin et al. 2008). Etwas nach 4000 v.h. datiert auch eine markante, bis heute anhaltende Verlagerung der polaren Waldgrenze auf der sibirischen Halbinsel nach Süden (Hantemirov und Shiyatov 2002).

Dass die im Alpenraum rekonstruierbare Umwelt- und Klimaentwicklung in die hemisphärische und globale Entwicklung eingebunden ist, wird auch bei der Analyse der Auswirkungen von extremen Vulkanereignissen deutlich. Der durch historische 
Beobachtungen gut dokumentierte Ausbruch des Tambora im Jahr 1815 hatte in manchen Gebieten der Nordhemisphäre ein „Jahr ohne Sommer“ (Harington 1992, Briffa et al. 1998) zur Folge. Auch im alpinen Raum waren die Auswirkungen stark, für die Bevölkerung in weiterer Folge teilweise sogar in Form einer Hungersnot spürbar (Fischer 1999). An den Hochlagenbäumen sind ab 1815 über mehrere Jahre hinweg extrem schmale Jahrringe, ja teilweise auch Jahrringausfälle zu beobachten. Dieser Zeitabschnitt gehört zu den extremsten Perioden während der letzten 9000 Jahre. Auch die Jahre um $540 \mathrm{n}$. Chr. weisen ähnlich schmale Jahrringe und gestörte Zuwachsmuster auf. Dies kann wiederum nicht nur im Alpenraum sondern auch an Jahrringdatensätzen aus dem übrigen eurasischen Bereich festgestellt werden (Larsen et al. 2008). Eine Analyse, unter Einbezug von Eisbohrkerndaten, belegt eine ähnliche, wenn nicht sogar größere Vulkanexplosion für diesen Zeitraum (Larsen et al. 2008).

Jahrringdaten von der alpinen Waldgrenze besitzen das Potential zur Rekonstruktion von sommerlichen Temperaturverhältnissen. Allerdings existieren solche dendroklimatologischen Analysen zur langfristigen Entwicklung der Sommertemperatur, basierend sowohl auf Jahrringbreite- als auch Spätholzdichtedaten, derzeit nur für die letzten rund 1000 Jahre (Büntgen et al. 2005, 2006). Eine Frequenzbandanalyse zeigt, dass sich die Verwendung densitometrischer Daten (Büntgen et al. 2006, Esper et al. 2007) im hochfrequenten Bereich gegenüber den Jahrringbreitenserien (Büntgen et al. 2005, Esper et al. 2007) in einer deutlich verbesserten Rekonstruktionsmöglichkeit niederschlägt; Jahrringbreitenchronologien wiederum spiegeln demgegenüber die langfristige Temperaturvariabilität besser wider. Insgesamt bestätigen die Rekonstruktionsergebnisse das an den Alpengletschern gewonnene grobe Bild einer vergleichsweise kühlen Periode in der zweiten Hälfte des vergangenen Jahrtausends sowie günstigeren Bedingungen für den größten Teil des Mittelalters. Vor allem wird auch der aktuelle Klimawandel, der vor allem durch instrumentelle Daten dokumentiert ist, mit diesen Proxy-Datensätzen klar nachgezeichnet (Büntgen et al. 2005, 2006, Esper et al. 2007).

\section{Dank}

Die Untersuchungen wurden vom Österreichischen Wissenschaftsfonds FWF (Projekte P13065-GEO, EXPICE P15828, HiMAT F3113-G02) und und der Europäische Kommission (ALP-IMP EVK-CT-2002-00148) gefördert. 


\section{Klimawandel in Österreich}

\section{Literatur}

Artmann A., 1949. Jahrringchronologische und -klimatologische Untersuchungen an der Zirbe und anderen Bäumen des Hochgebirges. Diss. Univ. München, 87 S.

Becker B., 1991. Dendrochronologische Datierung von Nadelhölzern (Tanne, Fichte, Kiefer) in Süddeutschland. In: Dendrochronologische Datierung von Nadelhölzern in der Hausforschung. Süddeutschland und angrenzende Gebiete. Internationales Symposium im Freilichtmuseum des Bezirks Oberbayern an der Glentleiten 18. Mai 1990. Freundeskreis Freilichtmuseum Südbayern, Grossweil, Germany. Schriftenreihe, Heft 10: 7-22.

Bircher W., 1982. Zur Gletscher- und Klimageschichte des Saastales. Unpubl. Diss. Univ. Zürich, $233 \mathrm{~S}$.

Brehme K., 1951. Jahrringchronologische und -klimatologische Untersuchungen an Hochgebirgslärchen des Berchtesgadener Landes. Zeitschrift für Weltwirtschaft 14(2/3): 65-80.

Briffa K.R., Jones P.D., Schweingruber F.H., Osborn T.J., 1998. Influence of volcanic eruptions on Northern Hemisphere summer temperature over the past 600 years. Nature 393: 450-455.

Büntgen U., Esper J., Frank D.C., Nicolussi K., Schmidhalter M., 2005. A 1052-year tree-ring proxy for Alpine summer temperatures. Climate Dynamics 25: 141-153.

Büntgen U., Frank D.C., Nievergelt D., Esper J., 2006. Summer temperature variations in the European Alps, A.D. 755-2004. Journal of Climate 19: 5606-5623.

Edouard J.L., Guibal F., Nicault A., Rathgeber C., Tessier L., Thomas A., Wicha, S., 2002. Arbres subfossiles (Pinus cembra, Pinus uncinata et Larix decidua) et évolution des forêts d'altitude dans les Alpes françaises au cours de l'Holocène. Approche dendrochronologique. Actes du colloque international «Equilibre et rupture dans les écosystèmes depuis 20000 ans en Europe de l'Ouest», 18-22 septembre 2000, Besançon, Presses Universitaires Francomtoises, 403-411.

Esper J., Büntgen U., Frank D., Pichler T., Nicolussi K., 2007. Updating the Tyrol tree-ring data set. In: Haneca K., Verheyen A., Beeckman H., Gärtner H., Helle G., Schleser G., (Hrsg.): TRACE - Tree Rings in Archaeology, Climatology and Ecology 5: 80-84.

Fischer K., 1999. Das Hungerjahr 1816/17 in Tirol und der Ausbruch des Vulkans Tambora. Der Schlern 73: 5-22.

Furrer G., Burga C., Gamper M., Holzhauser H., Maisch M., 1987. Zur Gletscher-, Vegetations- und Klimageschichte der Schweiz seit der Späteiszeit. Geographica Helvetica 42: 61-91.

Giertz-Siebenlist V., 1977. Der gegenwärtige Stand einer Jahrringchronologie aus dem obersten Ötztal. In: Frenzel B. (Hrsg.): Dendrochronologie und Postglaziale Klimaschwankungen in Europa. Erdwissenschaftliche Forschung 13: 25-27.

Grabner M., Gindl W., 2000. Neue Jahrringchronologien vom Dachstein. In: Mandl F. (Hrsg.): Alpen, Archäologie, Felsbildforschung V. Mitteilungen der ANISA 21/1-2: 20-30.

Grabner M., Wimmer R., Gindl W., Nicolussi K., 2001. A 3474-year alpine tree-ring record from the Dachstein, Austria. In: Tree Rings and People. International Conference on the Future of Dendrochronology. 22.-26.9.2001, Davos, Switzerland. Abstracts, 252-253.

Haas J.N., Richoz I., Tinner W., Wick L., 1998. Synchronous Holocene climatic oscillations recorded on the Swiss Plateau and at timberline in the Alps. The Holocene 8: 301-309. 
Hantemirov R.M., Shiyatov S.G., 2002. A continuous multimillennial ring-width chronology in Yamal, northwestern Siberia. The Holocene 12: 717-726.

Harington C.R. (Hrsg.) 1992. The Year Without a Summer? World Climate in 1816. Ottawa 1992.

Holzhauser, H., 1997. Fluctuations of the Grosser Aletsch Glacier and the Gorner Glacier during the last 3200 years, new results. In: Frenzel, B.(Hrsg.): Glacier fluctuations during the Holocene. Paläoklimaforschung / Palaeoclimate Research 24: 35-58.

Holzhauser H., Magny M., Zumbühl, H.J., 2005. Glacier and lake-level variations in west-central Europe over the last 3500 years. The Holocene 15: 789-801.

Hormes A., Müller B.U., Schlüchter C., 2001. The Alps with little ice: evidence for eight Holocene phases of reduced glacier extent in the Central Swiss Alps. The Holocene 11: 255-265.

Huber B., 1941. Aufbau einer mitteleuropäischen Jahrring-Chronologie. Mitteilungen Hermann Göring Akademie d. deutschen Forstwissenschaft 3: 137-142.

Jörin U.E., Stocker T.F., Schlüchter C., 2006. Multicentury glacier fluctuations in the Swiss Alps during the Holocene. The Holocene 16: 697-704.

Jörin, U. E., Nicolussi K., Fischer A., Stocker T.F., Schlüchter C., 2008. Holocene optimum events inferred from subglacial sediments at Tschierva Glacier, Eastern Swiss Alps. Quaternary Science Reviews 27/3-4: 337-350.

Kröpelin S., Verschuren D., Lézine A.-M., Eggermont H., Cocquyt C., Francus P., Cazet J.-P., Fagot M., Rumes B., Russell J.M., Darius F., Conley D.J., Schuster M., von Suchodoletz H., Engstrom D.R., 2008. Climate-Driven Ecosystem Succession in the Sahara: The Past 6000 Years. Science 320/ 5877: 765-768.

Larsen L.B., Vinther B.M., Briffa K.R., Melvin T.M., Clausen H.B., Jones P.D., Siggaard-Andersen M.-L., Hammer C.U., Eronen M., Grudd H., Gunnarson B.E., Hantemirov R.M., Naurzbaev M.M., Nicolussi K., 2008. New Ice Core Evidence for a Volcanic Cause of the A.D. 536 Dust-veil. Geophysical Resarch Letters 35, L04708, doi:10.1029/2007GL032450.

Nicolussi K., Patzelt G., 2001. Untersuchungen zur holozänen Gletscherentwicklung von Pasterze und Gepatschferner (Ostalpen). Zeitschrift für Gletscherkunde und Glazialgeologie 36: 1-87.

Nicolussi, K., Lumassegger, G., Patzelt, G., Pindur, P. and Schießling, P., 2004. Aufbau einer holozänen Hochlagen-Jahrring-Chronologie für die zentralen Ostalpen: Möglichkeiten und erste Ergebnisse. In: Innsbrucker Geographische Gesellschaft (Hrsg.): Innsbrucker Jahresbericht 2001/2002, 16: 114-136.

Nicolussi K., Kaufmann M., Patzelt G., van der Plicht J., Thurner A., 2005. Holocene tree-line variability in the Kauner Valley, Central Eastern Alps, indicated by dendrochronological analysis of living trees and subfossil logs. Vegetation History and Archaeobotany 14: 221-234.

Nicolussi K., Joerin U.E., Patzelt G., Stocker T.F, Thurner A., Schluechter C., 2008. Even smaller than now - evidence for long lasting glacier retreat periods in the Alps during the Holocene. Geophysical Research Abstracts, 10, EGU2008-A-04653.

Nicolussi K., Kaufmann M., Melvin T.M., van der Plicht J., Schießling P., Thurner A., in Druck. A 9111 year long conifer tree-ring chronology for the European Alps. The Holocene.

Oberhuber W., Kofler W., 2002. Dendroclimatological spring rainfall reconstruction for an inner Alpine dry valley. Theoretical and Applied Climatology 71: 97-106. 


\section{Klimawandel in Österreich}

Patzelt G., Bortenschlager S., 1973. Die postglazialen Gletscher- und Klimaschwankungen in der Venedigergruppe (Hohe Tauern, Ostalpen). Zeitschrift für Geomorphologie N.F., Suppl. Bd. 16: 25-72.

Patzelt G., 1977. Der zeitliche Ablauf und das Ausmaß postglazialer Klimaschwankungen in den Alpen . In: Frenzel B. (Hrsg.): Dendrochronologie und postglaziale Klimaschwankungen in Europa. Erdwissenschaftliche Forschung 13: 249-259.

Pichler T., Nicolussi K., Goldenberg G., in Druck. Evidence of prehistoric mining activities by means of dendrochronological analysis of wooden artefacts from the copper mine Kelchalm/Kitzbühel. Dendrochronologia.

Reimer P.J., Baillie M.G.L., Bard E., Bayliss A., Beck J.W., Bertrand C.J.H., Blackwell P.G., Buck C.E., Burr G.S., Cutler K.B., Damon P.E., Edwards R.L., Fairbanks R.G., Friedrich M., Guilderson T.P., Hogg A.G., Hughen K.A., Kromer B., Mccormac G., Manning S., Ramsey C.B., Reimer R.W., Remmele S., Southon J.R., Stuiver M., Talamo S., Taylor F.W., Van Der Plicht J., Weyhenmeyer C.E., 2004. IntCal04 terrestrial radiocarbon age calibration, 0-26 cal kyr BP. Radiocarbon 46/3: 1029-1058.

Reitmaier T., Nicolussi K., 2002. Ein hochmittelalterlicher Einbaum aus dem Obersee, Gemeinde St. Jakob / Defereggen (Osttirol), und die fischereiwirtschaftliche Nutzung alpiner Hochgebirgsseen in Tirol. NAU - Nachrichtenblatt Arbeitskreis Unterwasserarchäologie 9: 12-16.

Renner F., 1982. Beiträge zur Gletschergeschichte des Gotthardgebietes und dendroklimatologische Analysen an fossilen Hölzern. Diss. Univ. Zürich, 180 S.

Röthlisberger F., Haas P., Holzhauser H., Keller W., Bircher W., Renner F., 1980. Holocene Climatic Fluctuations - Radiocarbon Dating of Fossil Soils (fAh) and Woods from Moraines and Glaciers in the Alps. In: Geography in Switzerland. Geographica Helvetica 35/5: 21-52.

Tranquillini W., 1979. Physiological Ecology of the Alpine Timberline. Ecological Studies 31, BerlinHeidelberg-New York (Springer).

Schär E., Schweingruber F.H., 1987. Nacheiszeitliche Stammfunde aus Grächen im Wallis. Schweizerische Zeitschrift für das Forstwesen 138/6: 497-515.

Schär E., Schweingruber F.H., 1988. 4000 Years' forest development in pre-christian times near a mountain lake in the Alps. Dendrochronologia 6: 131-140.

Seifert M., 2000. Vor 3466 Jahren erbaut! Die Quellfassung von St. Moritz. Archäologie der Schweiz 23/2: 63-75. 


\title{
Klimaschwankungen und -trends des älteren Holozäns in den südlichen Niederen Tauern: multidisziplinäre Auswertung eines Sedimentkerns aus dem Oberen Landschitzsee (Lungau)
}

\author{
Schmidt R. ${ }^{1)}$, Kamenik C. ${ }^{2)}$, Kaiblinger C. ${ }^{1)}$, Tessadri R. ${ }^{3)}$ \\ 1) Institut für Limnologie, Österreichische Akademie der Wissenschaften, Mondsee, \\ Österreich, \\ 2) Oeschger Center for Climate Research \& Institut für Geographie, Universität Bern, \\ Schweiz \\ 3) Institut für Mineralogie und Petrographie, Universität Innsbruck, Österreich
}

\section{Abstract}

Early-Holocene climate changes and trends in the southern Niedere Tauern: a multiproxy study of a lake sediment core from Oberer Landschitzsee (Lungau)

Lake Oberer Landschitzsee (2.067 $\mathrm{m}$ a.s.l.) is a key site for the reconstruction of Holocene climate changes at the southern Austrian Central Alps (Niedere Tauern, Lungau). The lake is located in a climatically sensitive ecotone close to the alpine treeline. Its radiocarbon (AMS ${ }^{14} \mathrm{C}$ ) dated sediments have been studied intensively using a multi-proxy approach. Local glaciers, which originated from the Younger Dryas cold period, have disappeared during the early Holocene warming. Subsequently, Oberer Landschitzsee was affected by two cold and wet periods. The first started about 10.5 kyrs before present (BP) and culminated at ca. 10.2 kyrs BP. The second occurred between ca. 9.5 and 9.2 kyrs BP. From ca. 9.2 to 8.7 kyrs BP a period of lake summer warming separated these older climate oscillations from younger climate deteriorations between ca. 8.7 to 7.6 kyrs BP. The latter coincided with glacier advances in the nearby Hohe Tauern (Venediger, Frosnitz), whereas in the Niedere Tauern perennial snowfields developed, which kept summer lake water temperatures low. Prolonged summer warming and late autumn mixing characterized the following three warm periods (Holocene optimum events) between ca. 7.5 to $5.3 \mathrm{kyrs}$ BP, which were interrupted by two cold fluctuations. The snowfields and possibly permafrost in the catchment of the lake disappeared during the Holocene optimum events, resulting in distinct lake warming (principle of ultra-sensitive lakes). The interval between ca. 7 and 6 kyrs was more continental than the younger one from ca. 6 to 5.3 kyrs BP. The change in continentality could explain the lowering in timber/treeline. During the following climate oscillation at ca. 5 kyrs BP, pronounced wet and snowrich conditions altered with drier ones. The period from ca. 5 to 4 kyrs BP indicated strong seasonal divergence, most probably due to the increasing impact of the mediterranean winterrain climate on the area south of the Alps. In sum, the time-window from ca. 11.5 to $4 \mathrm{kyrs}$ $\mathrm{BP}$ indicated (i) increasing temperatures with a plateau between 7.5 and $5.3 \mathrm{kyrs}$ BP, (ii) a 
long-term trend of declining continentality, probably due to the increasing influence of the mediterranean winter-rain climate, (iii) climate oscillations with a periodicity of approximately 1000 years, (iv) two marked, well-known, acyclic, short-term cold fluctuations: the 8.2 kyrs cold event and the pre-boreal oscillation at about 11.3 kyrs BP.

\section{Einleitung}

Alpenseen stellen wichtige Klimaarchive vor allem aus zweierlei Gründen dar: (1) schon geringe Temperaturveränderungen führen zu großen hydrochemischen, physikalischen und biologischen Veränderungen (Sommaruga-Wögrath et al. 1997, Psenner \& Lackner 2006); (2) Klimaschwankungen sind in der alpinen Region deutlich ausgeprägt. So liegt die rezente Erwärmung in den Alpen deutlich über dem globalen Durchschnitt (u.a. Beniston et al. 1997, Auer et al. 2007). Die Informationen über Klima- und Umweltveränderungen bleiben in den Ablagerungen von Alpenseen gespeichert und sind für die Paläolimnologie zugänglich.

Die Niederen Tauern in den österreichischen Zentralalpen weisen auf engem Raum eine größere Zahl kleinerer Seen mit relativ kleinen Einzugsgebieten auf. In 45 dieser Seen zwischen ca. 1500 und 2300 m wurden über zwei Jahre (19981999) die oberflächennahen Wassertemperaturen mittels automatischer Temperaturmessgeräte gemessen. Diese Thermistor-Messdaten wurden für die Bestimmung der Temperaturoptima und -toleranzen von Kieselalgen (Diatomeen) verwendet (Schmidt et al. 2004a, b). Diese Algen bleiben aufgrund ihrer robusten silikatischen Zellwand zumeist gut in Seesedimenten erhalten. Für die Rekonstruktion von klimaabhängigen Variablen wurde mittels sogenannter Transferfunktionen, basierend auf der in der Paläolimnologie etablierten Methode gewichteter Mittel, Kalibration und Regression (Zusammenfassung bei Birks 1998), der Zusammenhang zwischen Wassertemperatur und Algenzusammensetzung modelliert. Unter diesen Modellen wies der „Zeitpunkt der Herbstdurchmischung“ die höchste Vorhersagequalität auf. Dies dürfte damit zusammenhängen, dass der indirekte Einfluss der Temperatur auf Diatomeen über den Weg der Durchmischung, woran neben Temperatur noch andere Einflussgrößen wie Wind und Veränderungen im Chemismus beteiligt sein können, größer ist als der Temperatureinfluss alleine. Mit Hilfe dieser Transferfunktion wurde nun der Zeitpunkt der Herbstdurchmischung für den Zeitraum von ca. 11.500 bis 4000 Jahre v. h. aus den fossilen Kieselalgen eines Sedimentkerns des Oberen Landschitzsees berechnet (Schmidt et al. 2004a). Aus den Zeitpunkten der Herbstdurchmischung und dem höhenstufenabhängigen Temperaturgradienten in 
den Kalibrationsseen der Niederen Tauern wurden letztlich die Temperatur-Anomalien geschätzt (zur genauen Methode siehe Schmidt et al. 2007).

Der Obere Landschitzsee ist ein kleiner (ca. 8,9 ha), 13,6 m tiefer, nährstoffarmer (oligotropher) See auf der Südabdachung der Niederen Tauern (Lungau) (Abb. 1).

Abb. 1. Oberer Landschitzsee, Niedere Tauern, Lungau, aus der Luft, von Süden (Foto R. Schmidt).

Fig. 1. Aerial view of Oberer Landschitzsee, Niedere Tauern, Lungau, from the south (Photograph R. Schmidt).

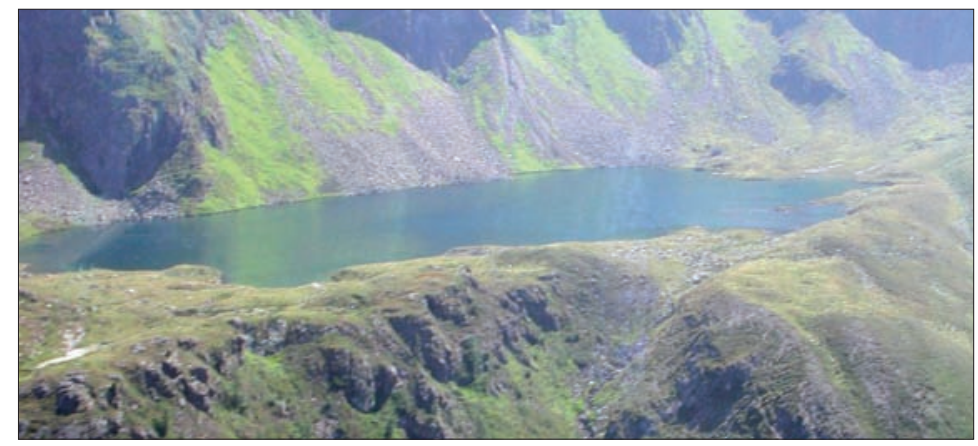

Der See liegt karartig auf $2.067 \mathrm{~m}$ Höhe etwas über der Baumgrenze, die von der Zirbe (Pinus cembra) gebildet wird. Der Sedimentkern (ObLAN) aus dem tiefsten Beckenbereich wurde mittels der Radiokarbonmethode (AMS ${ }^{14} \mathrm{C}$ ) datiert. Aus den kalibrierten ${ }^{14} \mathrm{C}$-Daten wurde eine Tiefen-Altersmodell in Jahren vor heute $(=\mathrm{v}$. h. $)$ erstellt. Da bei der verwendeten ${ }^{14} \mathrm{C}$-Methode Datierungsunschärfen unvermeidlich sind ("floating chronology"), können diese nur Annäherungswerte sein. Neben der quantitativen Rekonstruktion des Zeitpunkts der Herbstdurchmischung wurden noch Pollen, Mineralogie und Geochemie zu einem multidisziplinären Ansatz vereinigt (Schmidt et al. 2006). Ein solcher ist hilfreich, um die einzelnen Signale der Klimaindikatoren zu einem Gesamtbild zusammenzufügen und damit die Interpretation abzusichern. Die folgende Darstellung gibt eine Zusammenfassung der Ergebnisse von Schmidt et al. (2004a und 2006).

\section{Klimarekonstruktion des älteren Holozäns}

Da nur holozäne Ablagerungen (letzten ca. 11.500 Jahre) im Sedimentkern erfasst wurden, dürfte der See noch während der Jüngeren Dryas, der letzten markanten Gletschervorstoßphase am Ende des Spätglazials, vom Gletschereis bedeckt gewesen sein. Die früh-Holozäne Warmphase fällt in die Pollenzone des sogenannten Präboreals. Der Temperaturanstieg und die wahrscheinlich kontinentalen $=$ warme 
und trockene Sommer, kalte und niederschlagsarme Winter) Verhältnisse dürften, im Gegensatz zu den heute noch stark vergletscherten Hohen Tauern, zum Abschmelzen der letzten Gletscher im Untersuchungsgebiet geführt haben. Auf diese, mit Ausnahme einer kurzen Fluktuation (siehe unten), warme Periode folgte um ca. 10.500 v. h. eine kühle und niederschlagsreiche Phase, die um ca. 10.200 v. h. kulminierte. Auch in Folge bis ca. 9.200 v. h. verharrte der See auf einem niedrigen sommerlichen Temperaturniveau, wenn auch eine schwache Zweiteilung angedeutet ist. Sucht man nach bekannten Gletschervorstößen in den Ostalpen aus dieser Zeit (Nicolussi \& Patzelt 2000), so bietet sich unter Berücksichtigung von Datierungsunschärfen die Venediger Schwankung der Hohen Tauern an (Patzelt \& Bortenschlager 1973). Schneereiche Verhältnisse dürften zu der Ausbildung ausdauernder Schneefelder im Einzugsgebiet des Oberen Landschitzsees geführt haben. Diese waren wahrscheinlich dafür verantwortlich, dass sich auch während der folgenden Warmphase zwischen ca. 9.200 und 8.600 v. h. der See nur mäßig erwärmte, wie aus den abgeleiteten Zeitpunkten der Herbstdurchmischung und den daraus errechneten Temperaturanomalien geschlossen werden kann. Diese Hypothese wird durch die sedimentologischen Analysen des Kerns bestätigt (Schmidt et al. 2006). Erhöhte Quarz- und Sandanteile lassen auf Schmelzwässereinflüsse schließen. Auf diese Erwärmung zwischen ca. 9.200 und 8.700 v. h., die nach Nicolussi et al. (2005) zu einer hohen Waldgrenze, d.h. höher als 1980, führte, folgte wieder eine klimatisch instabile Periode, die bis ca. 7.600 v. h. andauerte. Diese schließt auch die kurzfristige Kälteschwankung um 8.200 v. h. ein. Diese Kälteschwankung lässt sich über weite Teile der Nordhemisphäre verfolgen, wie etwa in den Sauerstoff-Isotopen $\left(\delta^{18} \mathrm{O}\right)$ aus Eiskernen Grönlands (Alley et al. 2002) oder biogenen Karbonaten des Ammersees in Bayern (von Grafenstein et al. 1998). Da es sich um eine relativ kurzfristige Schwankung handelte $(<100$ Jahre), ist jedoch für deren Nachweis eine entsprechende zeitliche Auflösung notwendig. Im Oberen Landschitzsee zeigt sich diese Schwankung u.a. in der Mineralzusammensetzung und im Pollenbild. Der auffallend geringe Quarzanteil und die verminderte Blühintensität der Grünerle (Alnus viridis) könnten auf eine erhöhte Schneeakkumulation und längere Schneedauer hinweisen, während es während der folgenden Erwärmung gerade umgekehrt war. Dies deckt sich mit Befunden aus anderen Gebieten (so u.a. auch der Pyrenäen, Pla \& Catalan 2005), die ebenfalls auf einen raschen Wechsel zwischen kalt und warm hinweisen. Kurzfristige abrupte Fluktuationen der Strontium-Werte und anderer geogener Elemente wie z. B. Rubidium und Titan könnten, ähnlich wie während der sogenannten Präborealen Oszillation (ca. 11.300 v. h., Björck et al. 1997), durch einen raschen Wechsel zwischen Schneeakkumulation und Abschmelzen verursacht 
sein. Diese Kälteschwankung scheint jedoch nur eine Episode innerhalb von zwei in ObLAN aufeinanderfolgenden Kälteperioden zwischen ca. 8.600 und 7.600 v. h. gewesen zu sein. Dies deckt sich mit den Ergebnissen von Magny (2004) aus dem nord-westlichen Randbereich der Alpen, der im vergleichbaren Zeitraum zwei nahe aneinander liegende kalte und feuchte Phasen (Phasen 11 und 12 nach Magny 2004) mit ausgeprägten Seespiegelhochständen nachweist. Inwieweit diese Serie von kalten und feuchten Klimaschwankungen mit der Frosnitz Schwankung (Patzelt \& Bortenschlager 1973) übereinstimmt, die in den Hohen Tauern (Venediger Gruppe) zu markanten Gletscherhochständen geführt hat, ist aufgrund von Datierungsunschärfen noch fraglich (Nicolussi \& Patzelt 2000). Auch im Adriaraum und nördlichen Mittelmeer gab es zwischen ca. 8.600 und 7.600 v. h. heute besonders feuchte, auch als Pluviale bezeichnete Phasen, die von kurzfristigen Trockenperioden unterbrochen wurden (u.a. Kallel et al. 1996, Wunsam et al. 1999, Aritztegui et al. 2000).

Abb. 2: Aus den fossilen Kieselalgen (Diatomeen) des Sedimentkerns Oberer Landschitzsee (südliche Zentralalpen) und einem Kalibrierdatensatz, von 40 Seen der Niederen Tauern abgeleitete Zeitpunkte der Herbstdurchmischung (in Kalendertagen) und die Temperatur-Anomalien gegenüber beute (1998/99). Die großen schwarzen Pfeile kennzeichnen die größeren Kälteschwankungen: $1=$ Schwankungen von ca. 10.500 bis $9.200 v \mathrm{~h}$. (Venediger?), 2 = Schwankungen von ca. 8.600 bis

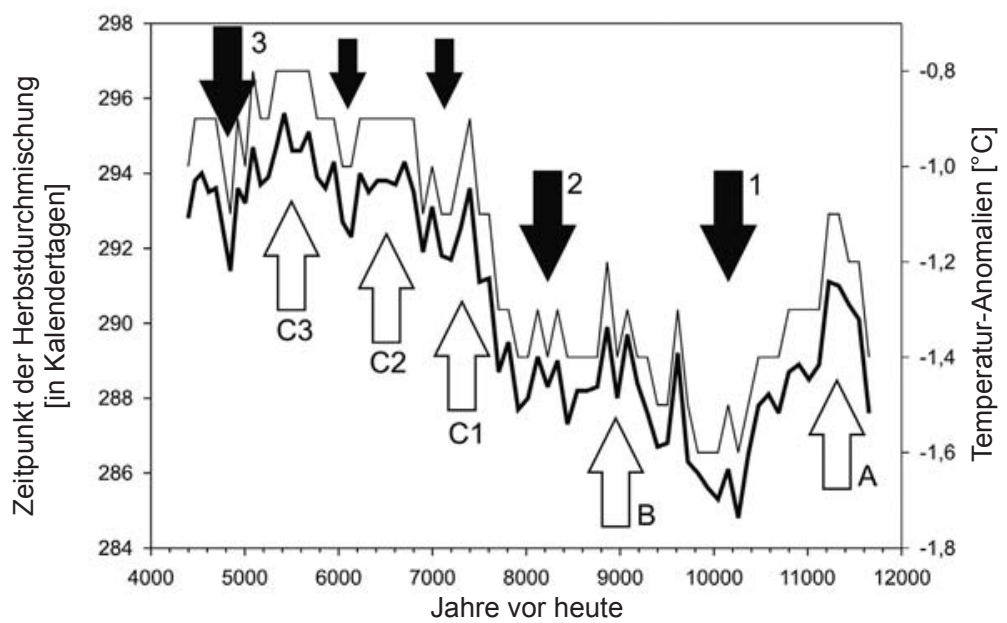

7.600 v.h. (Frosnitz?), 3 = Schwankungen um 5.000 v. h. (Rotmoos?). Die kleineren schwarzen Pfeile kennzeichnen zwei untergeordnete Schwankungen, die das „Holozäne Klimaoptimum" unterteilen. Die weissen Pfeile kennzeichnen die Warmperioden: $A=$ Früh-Holozäne Warmperiode; $B=$ Warmperiode um 9.000 v. h., $C=$ Warmperioden 1 - 3 ("Holocene optimum events") zwischen ca. 7.500 und 5.300 v. h., zusammengefasst als sogenanntes „Holozänes Klimaoptimum" (nach Schmidt et al. 2004, verändert).

Fig. 2: Dates of autumn mixing (in Julian days) and temperature anomalies (reference 1998/99) in a sediment core of Oberer Landschitzsee (southern Central Alps) inferred from fossil calibrated diatoms (based on 40 training set lakes in the Niedere Tauern). The larger black arrows indicate the major cold periods $1-3$, the smaller black arrows two cold climate fluctuations separating the so-called "Holocene climatic optimum" into three "Holocene optimum events". The white arrows indicate the major early-Holocene warm periods $\mathrm{A}-\mathrm{C}$. 
Zwischen ca. 7.500 und 7.000 v. h. setzte eine, wahrscheinlich durch einen Rückschlag unterbrochene, markante Klimaerwärmung ein. Um die Vorgänge von damals besser verstehen zu können, stellten Thompson et al. (2005) die Wassertemperaturen der 45 Seen der Niederen Tauern in Relation zu der für die jeweilige Meereshöhe der Seen zu erwartenden Lufttemperatur. Dabei fielen einzelne Seen auf (besonders jedoch der Moaralmsee auf der Nordseite der Niederen Tauern im Ennstal), die deutlich unterkühlt sind. Mögliche Ursachen sind kalte Grundwasserströme und/oder ausdauernde Schneefelder im Einzugsgebiet dieser Seen. Thompson et al. (2005) sprechen von ultra-sensitiven Seen, da sich diese Seen nach Wegfall der kühlenden Einflüsse und in Angleichung an die Lufttemperatur, wie etwa im Zuge einer Klimaerwärmung, überproportional erwärmen würden. Analog führte das Verschwinden ausdauernder Schneefelder und damit verbundener Schmelzwässereinflüsse am Oberen Landschitzsee vor 7.000 Jahren zu einer deutlichen Erwärmung des Sees sowie späten Durchmischungszeiten im Herbst. Der Zeitraum zwischen ca. 7.500 und 5.300 v. h., der auch als ,postglaziale Wärmezeit“" oder „, holozänes bzw. postglaziales Klimaoptimum" bezeichnet wurde, ist in den Alpen durch hohe Waldgrenzen und überwiegend geringe Gletscherausdehnung gekennzeichnet (u.a. Nicolussi \& Patzelt 2000, Hormes et al. 2001, Tinner \& Theurillat 2003). Im Kaunertal, Tirol, zeigen Holzuntersuchungen von Nicolussi et al. (2005), dass in diesem Zeitabschnitt auch die Baumgrenze von 2000 A.D. überschritten wurde. Da die Warmzeit aber von Kälterückschlägen unterbrochen wurde, wird auch von „Holocene optimum events“ gesprochen (Joerin et al. 2007). Im Oberen Landschitzsee ist dieser Abschnitt durch zwei kleinere Schwankungen dreigeteilt. Obwohl diese innerhalb der Schwankungsbreite des Modellfehlers liegen, stimmt jene um 6.000 v. h., die auch in einem Sedimentkern des Unteren Landschitzsees nachgewiesen wurde (Schmidt et al. 2002), gut mit den dendrochronologischen Ergebnissen überein (Nicolussi et al. 2005). Aufgrund der geochemisch/mineralogischen Befunde (Schmidt et al. 2006) war die Warmperiode zwischen 7.000 und der Klimaschwankung um ca. 6.000 v. h. wahrscheinlich kontinentaler (schneeärmer) als die jüngere Warmphase zwischen ca. 6.000 und $5.000 \mathrm{v}$. h. Möglicherweise führten die schneereicheren Bedingungen während der jüngeren Warmphase zu einem Absenken der Waldgrenze (Nicolussi et al. 2005). Aufgrund unterschiedlicher Bewertungen des Niederschlagseinflusses (von $-250 \mathrm{~mm} / \mathrm{Jahr}$ bis $+250 \mathrm{~mm} / \mathrm{Jahr}$ ) schwanken dementsprechend auch die Temperaturschätzungen für das „Holozäne Klimaoptimum“ zwischen $+1{ }^{\circ} \mathrm{C}$ und $+2,5^{\circ} \mathrm{C}$ (Joerin et al. 2007). Demzufolge würde das Maximum der abgeleiteten Temperatur-Anomalien des Oberen Landschitzsees für diese Zeit, das unter dem Referenzwert von 1998/99 liegt, den tatsächlichen Wert unterschätzen. 
Um 5.000 v. h. deuten Fluktuationen einer Vielzahl geochemischer und mineralogischer Kenngrößen auf einen raschen Wechsel zwischen kühlen, schneereichen Abschnitten und wärmeren Phasen mit verstärktem Abschmelzen hin. Eine stärkere Erosionstätigkeit wird für diese Zeit auch aus den Hohen Tauern berichtet (Fritz \& Ucic 2001). In diese Zeit fallen die sogenannten Rotmoos Schwankungen in Tirol (Gams 1962, Bortenschlager 1970). Auch der Tiroler Eismann vom Hauslabjoch ist in diese Zeit um 5.300 v. h. datiert (Bortenschlager \& Oeggl 2000) und seine Erhaltung könnte mit diesen abrupten Kälteschüben in Zusammenhang stehen. Der Abschnitt von ca. 5.000 bis 4.000 Jahren v. h. deutet in ObLAN auf starke saisonale Unterschiede zwischen niederschlagsreichen Wintern (Herbst bis Frühling) und warmen und trockenen Sommern hin. Als Arbeitshypothese wurde eine Zunahme des Einflusses des mediterranen Winterregenklimas auf den südlichen Alpenraum postuliert, der möglicherweise mit der holozänen Transgression der Adria (Correggiari et al. 1996) im Zuge des globalen Meeresspiegelanstieges im Zusammenhang steht. Um ca. 4.000 v. h. erreichte jedenfalls die Steineiche (Quercusilex), der Charakterbaum der Eu-Mediterranen Zone, auf ihrer Holozänen Ausbreitung in Europa von Süd nach Nord (u.a. Jahns 1991, Wunsam et al. 1999, Jalut et al. 2000, DrescherSchneider et al. 2006) ihre heutige Nordgrenze im Adriaraum (Schmidt et al. 2000). Im Zuge dieser langfristigen Klimaänderung dürften die Winter, zumindest in den südlichen Alpen, schneereicher geworden sein.

\section{Schlussfolgerungen}

Der Obere Landschitzsee auf der Südabdachung der Zentralalpen (Lungau) zeigt im Zeitfenster von ca. 11.500 bis 4.000 v. h.: (i) Längerfristige Wärmeperioden: zu Beginn des Holozäns, um ca. 9.000 v. h., sowie das mehrphasige ("Holocene optimum events") sogenannte „Holozäne Klimaoptimum“ zwischen ca. 7.500 und 5.300 v. h; (ii) kalte Klimaschwankungen mit einer ungefähren Periodizität von 1000 Jahren (um \pm 10, 9, 8, 7, 6, 5000 v. h.). Zwischen 8.600 und 7.600 und um 5.000 v.h. (Rotmoos?) scheinen diese Klimaschwankungen zudem besonders feucht gewesen zu sein. Eine Südverlagerung atlantischer Tiefdrucksysteme könnte sowohl die niederschlagsreichen Klimaschwankungen in den südlichen Alpen als auch die wahrscheinlich zeitgleichen pluvialen Abschnitte des Mittelmeerraumes zwischen ca. 8.600 und 7.600 v. h. erklären. Das Prinzip der geteilten Kälteschwankungen, wie für das Spätglazial postuliert, kann auch auf die großen (globalen?) Klimaschwankungen des älteren Holozäns übertragen werden; (iii) zwei markante, kurzfristige (azyklische), 
Kälteerreignisse, bekannt als Präboreale Oszillation und 8.200er Kälte-Event; sowie (iv) einen langfristigen Wechsel von kontinentalen Klimaverhältnissen zu mediterranen, winterregen-beeinflussten Klimaten in den südlichen Alpen.

In der früh-Holozänen Warmphase dürften die Gletscher der Niederen Tauern, die sich in der ausklingenden Späteiszeit (Jüngere Dryas) gebildet haben, weitgehend abgeschmolzen sein. Während der folgenden kalten und niederschlagsreichen Klimaschwankungen des älteren Holozäns, die in den Hohen Tauern erneut zu Gletschervorstößen führten (Venediger, Frosnitz), dürften sich in Karen der Niederen Tauern, wie am Oberen Landschitzsee, ausdauernde Schneefelder gebildet haben. Schmelzwässer aus diesen Schneefeldern waren wahrscheinlich dafür verantwortlich, dass sich der See auch während dazwischenliegender Warmphasen nur mäßig erwärmte und damit nicht mit der Lufttemperatur im Gleichgewicht stand. Mit dem Verschwinden der Schneefelder zu Beginn des „Holozänen Klimaoptimums“ um ca. 7000 v. h. erwärmte sich der Obere Landschitzsee dagegen deutlich (Prinzip der ultra-sensitiven Seen). Dieser Abschnitt in der Entwicklungsgeschichte des Oberen Landschitzsees kann somit als Beispiel für sich neubildende Alpenseen im Zuge des Gletscherrückzuges unter der heutigen Klimaerwärmung dienen.

\section{Dank}

Die Untersuchungen wurden durch Mittel des Österr. Wissenschaftsfonds (FWF, Projekt P14912-B06) und des IGBP-Global Change programme (IGBP-29/20032004) der Österr. Akademie der Wissenschaften und des Wissenschaftsministeriums gefördert. Wir danken weiters den in den einzelnen Publikationen benannten Personen.

\section{Literatur:}

Auer, I., R. Boehm, A. Jurkovic, W. Lipa, A. Orlik, R. Potzmann, W. Schoener, M. Ungersboeck, C. Matulla, K. Briffa, P. Jones, D. Efhymiadis, M. Brunetti, T. Nanni, M. Maugeri, L. Mercalli, O. Mestre, J.-M. Moisselin, M. Begert, G. Mueller-Westermeier, V. Kveton, O. Bochnicek, P. Stastny, M. Lapin, S. Szalai, T. Szentimrey, T. Cegnar, M. Dolinar, M. Gajic-Capka, K. Zaninovic, Z. Majstorovic, E. Nieplova, 2005: HISTALP Historical Instrumental Climatological Surface Time Series of the Greater Alpine Region 2007, International Journal of Climatology, 17: 14-46

Alley R.B., Watts W.A., McGee E., Huntley B. 2002. Holocene climate instability: a prominent, widespread event 8200 yrs ago. Geology 25: 483-486. 
Aritztegui D., Asioli A., Low J.J., Trincardi F., Viglioti L., Tamburin F., Chondrogianni C., Accorsi C.A., Bandini Mazzanti M., Mercuri A.M., der Kaars S., McKenzie J.A., Oldfield F. 2000. Palaeoclimate and the formation of sapropel S1: inferences from Late Quaternary lacustrine and marine sequences in the central Mediterranean region. Palaeogeo., Palaeoclim., Palaeecol. 158: 215-240.

Beniston M., Diaz H.F., Bradley R.S. 1997. Climatic change at high elevation sites: An overview. In: Diaz H.F., Beniston M., Bradley R.S. (Eds.), Climatic Change at high elevation sites, Kluwer Academic Publishers, Dordrecht, 1-16.

Birks H.J.B. 1998. Numerical tools in palaeolimnology - Progress, potentialities, and problems. J. Paleolimnol. 20: 307-332.

Björck S., Rundgren M., Ingolfsson O., Funder S. 1997. The Preboreal oscillation around the Nordic Seas: terrestrial and lacustrine responses. J. Quat. Sci. 12: 455-465.

Bortenschlager S. 1970. Waldgrenz- und Klimaschwankungen im pollenanalytischen Bild des Gurgler Rotmooses. Mitt. Ostalp.-dinar. Ges. Vegetationskde. 11: 19-26.

Bortenschlager S., Oeggl K. 2000. The man in the ice. IV the iceman and his natural environment. Springer Humanbiology, Vienna, Austria, pp 164.

Correggiari A., Roveri M., Trincardi F. 1996. Late Pleistocene and Holocene evolution of the North Adriatic Sea. Il Quaternario 9: 697-704.

Drescher-Schneider R., Beaulieu J.-L., Magny M., Walter-Simonnet A.-V., Bossuet G., Millet L., Brugiapaglia E., Drescher A. 2006. Vegetation history, climate and human impact over the last 15,000 years at Lago dell'Accesa (Tuscany, Central Italy). Veget. Hist. Archaeobot. 16: 279-299.

Fritz A., Ucik F.H. 2001. Klimageschichte der Hohen Tauern. Spätwürmzeitliche und postglaziale Klima- und Vegetationsentwicklung in den südlichen Hohen Tauern (Ostalpen, Kärnten). Nationalpark Hohe Tauern, Sonderband 3, Kärntner Nationalparkfonds, pp 99.

Gams H. 1962. Das Gurgler Rotmoos und seine Stellung innerhalb der Gebirgsmoore. Veröff. Geobot. Inst. Rübel, Zürich 37: 74-82.

Hormes A., Müller B.U., Schlüchter C. 2001. The Alps with little ice: evidence for eight Holocene phases of reduced glacier extent in the Central Swiss Alps. Holocene 11: 255-265.

Jahns S. 1991. Untersuchungen über die Vegetationsgeschichte von Süddalmatien und Südgriechenland. Diss. Cuvilier Verlag, Georg-August-Univ. Göttingen, ISBN 3-928815-06-7, pp 144.

Jalut G., Esteban Amat A., Bonnet L., Gauquelin T., Fontugne M. 2000. Holocene climatic changes in the western Mediterranean from south-east France to south-east Spain. Palaeogeo., Palaeoclim., Palaeoecol. 160: 255-290.

Joerin U.E., Nicolussi K., Fischer A., Stocker T.F., Schlüchter C. 2007. Holocene optimum events inferred from subglacial sediments at Tschierva Glacier, Eastern Swiss Alps. Quat. Sci. Rev., in press.

Kallel N., Paterne M., Dupplessy J.C., Vergnaud-Grazzini C., Pujol C., Labeyrie L., Arnold M., Fontugne M., Pierre C. 1996. Enhanced rainfall in the Mediterranean region during the last sapropel event. Ocean. Acta 20: 697-712.

Magny M. 2004. Holocene climate variability as reflected by Mid-European lake-level fluctuations and its probable impact on pre-historic human settlements. Quat. Int. 113: 65-79.

Nicolussi K., Patzelt G. 2000. Untersuchungen zur Holozänen Gletscherentwicklung von Pasterze und Gepatschferner (Ostalpen). Z. Gletscherkde. Glazialgeol. 36: 1-87. 


\section{Klimawandel in Österreich}

Nicolussi K., Kaufmann F., Patzelt G., van der Pflicht J., Thurner A. 2005. Holocene tree-line variability in the Kauner Valley, Central Eastern Alps indicated by dendrochronological analysis of living trees and subfossil logs. Veget. Hist. Archaeobot. 14: 221-234.

Patzelt G., Bortenschlager S. 1973. Die postglazialen Gletscher- und Klimaschwankungen in der Venedigergruppe (Hohe Tauern, Ostalpen). Z. Gletscherkde. Glazialmorphol. N.F. Suppl. 16: 25-72.

Pla S., Catalan J. 2005. Chrysophyte cysts from lake sediments reveal the submillenial winter/spring climate variability in the northwestern Mediterranean region throughout the Holocene. Clim. Dynam. 24: 263-278.

Psenner R., Lackner R. 2006. Die Alpen im Jahr 2020. alpine space - man \& environment, Innsbruck University Press, 121 pp.

Schmidt R., Müller J., Drescher-Schneider R., Krisai R., Szeroczynska K., Baric A. 2000. Changes in lake level and trophy at Lake Vrana, a large karstic lake on the Island of Cres (Croatia), with respect to palaeoclimate and anthropogenic impacts during the last approx. 16,000 years. J. Limnol. 59: 113-130.

Schmidt R., Kamenik C., Kaiblinger C., Hetzel M. 2004a. Tracking Holocene environmental changes in an alpine lake sediment core: application of regional diatom calibration, geochemistry, and pollen. J. Paleolimnol. 32: 177-196.

Schmidt R., Kamenik C., Lange-Bertalot H., Klee R. 2004b. Fragilaria and Staurosira (Bacillariophyceae) from sediment surfaces of 40 lakes in the Austrian Alps in relation to environmental variables, and their potential for palaeoclimatology. J. Limnol. 63: 171-189.

Schmidt R., Kamenik C., Tessadri R., Koinig K.A. 2006. Climatic changes from 12,000 to 4,000 years ago in the Austrian Central Alps tracked by sedimentological and biological proxies of a lake sediment core. J. Paleolimnol. 35: 491-505.

Schmidt R., Kamenik C., Roth M. 2007. Siliceous algae-based seasonal temperature inference and indicator pollen tracking ca. 4,000 years of climate/land use dependency in the southern Austrian Alps. J. Paleolimnol. 38: 541-554.

Schmidt R., Koinig K.A., Thompson R., Kamenik C. 2002. A multi proxy core study of the last 7000 years of climate and alpine land-use impacts on an Austrian mountain lake (Unterer Landschitzsee, Niedere Tauern). Palaeogeo., Palaeoclim., Palaeoecol. 187: 101-120.

Sommaruga-Wögrath S., Koinig K.A., Schmidt R., Sommaruga R., Tessadri R., Psenner R. 1997. Temperature effects on the acidity of remote alpine lakes. Nature 387: 64-70.

Tinner W., Theurillat J.P.. 2003. Uppermost limit, extent, and fluctuations of the timberline and treeline ecoline in the Swiss Central Alps during the past 11500 Years. Arch. Alp. Res. 35: 158-169.

Thompson R., Kamenik C., Schmidt R. 2005. Ultra-sensitive Alpine lakes and climate change. J. Limnol. 64: 139-152.

von Grafenstein U. 1998. The cold event 8200 years ago documented in oxygen isotope records of precipitation in Europe and Greenland. Clim. Dynam. 14: 73-81.

Wunsam S., Schmidt R., Müller J. 1999. Holocene lake development of two Dalmatian lagoons (Malo and Veliko Jezero, Isle of Mljet) in respect to changes in Adriatic sea level and climate. Palaeogeo., Palaeoclim., Palaeoecol. 146: 251-281. 


\title{
Wie alt sind Blockgletscher in den Österreichischen Alpen? Das Beispiel der Blockgletscher im Dösener Tal, Ankogelgruppe, datiert mit Hilfe der Schmidt-Hammer Methode
}

\author{
Andreas Kellerer-Pirklbauer \\ Institut für Geographie und Raumforschung, Karl-Franzens-Universität Graz
}

Abstract

What is the age of rock glaciers in the Austrian Alps? The example of the rock glaciers in the Dösen Valley, Ankogel Mountains, dated by the Schmidt-hammer method.

Active rock glaciers are common large-scale flow features originating from thick debris accumulations (talus and/or till) in high-relief environments which are under permafrost conditions for a substantial period of time. Rock glaciers are characteristic landforms in mountain permafrost environments. More than one thousand rock glaciers formed in the Austrian Alps after the retreat of the Pleistocene glacier systems. Today, most of these rock glaciers are relict features without any ice content but still with the typical surface topography which makes them easy to identify in the field. These relict features indicate former permafrost conditions. Rock glaciers need hundreds to thousands of years to form, thus, datings on rock glaciers provide valuable information on palaeoclimatic conditions. This paper presents datings on three rock glaciers (containing permafrost) in the Dösen Valley, Ankogel Mountains, Hohe Tauern Range, derived with the Schmidt-hammer method. Results of this approach as well as additional palaeoclimatic information show that all three rock glaciers are old landforms. Their formation started already in the early Holocene. The largest of the three rock glaciers was constantly active during the last (at least) 8400 years. This indicates that permafrost conditions were present during this entire period in the Dösen Valley at elevations above $2350 \mathrm{~m}$ a.s.l and at coarse debris slopes facing west to north. Similar long formation periods can be assumed for many of the other Austrian rock glaciers. Additional relative and absolute datings on rock glaciers in Austria could help to validate the results from the Dösen valley as well as improve the understanding of rock glacier formation in the Austria during the last thousands of years.

\section{Zusammenfassung}

Blockgletscher sind lappen- bis zungenförmige Eis- und Schuttgemische, die sich lavastromartig langsam hang- oder talabwärts bewegen. Sie gelten als typisches Landschaftselement des alpinen Permafrosts (Dauerfrost). In den österreichischen Alpen haben sich nach dem Rückzug der Gletscher am dem Ende der letzten Eiszeit über tausend solcher Blockgletscherformen gebildet. Die meisten dieser Blockgletscher sind heute eisfrei und somit Zeugnis früherer Permafrostbedingungen. Bedenkt man, dass Blockgletscher für ihre Entwicklung hunderte bis tausende von Jahren mit Permafrostbedingungen benötigen, wird klar, dass Datierungen mit Bezug zur

alpine space - man \& environment, vol. 6: Klimawandel in Österreich 
Entstehungsgeschichte zusätzliche, wertvolle Hinweise über das Paläoklima geben können. Dieser Beitrag präsentiert Ergebnisse von Schmidt-Hammer Untersuchungen an drei Blockgletschern (mit Permafrost) im hinteren Dösener Tal, Ankogelgruppe, Hohe Tauern. Mit Hilfe dieser relativen Datierungsmethode und zusätzlichen paläoklimatischen Informationen wird gezeigt, dass die drei Blockgletscher alte Landschaftselemente sind, deren Entwicklung bereits im frühen Holozän begonnen hat. So hat sich der größte der drei untersuchten Blockgletscher wahrscheinlich kontinuierlich über einen Zeitraum von mindestens 8400 Jahren entwickelt. Dies bedeutet u.a., dass in diesem Zeitraum ab einer Seehöhe von $2350 \mathrm{~m}$ auf West- und Nordhängen mit Grobschuttauflage im Dösener Tal Permafrostbedingungen vorherrschend waren. Ähnlich lange Entwicklungsgeschichten sind für viele andere Blockgletscher in Österreich denkbar und weitere Datierungen (relative wie absolute) sind anzustreben, um einerseits die Ergebnisse dieser Untersuchung zu prüfen und andererseits die Entwicklung der österreichischen Blockgletscher im Laufe der letzten Jahrtausende besser zu verstehen.

\section{Einleitung}

Aktive Blockgletscher sind lappen- bis zungenförmige Eis- und Schuttgemische, die sich mit Geschwindigkeiten von wenigen Zentimetern bis Metern pro Jahr hangoder talabwärts bewegen. Solche Kriechformen sind charakteristisch für den alpinen Permafrost (oder Dauerfrost) und weisen eine charakteristische Oberflächenformung (z.B. steile und labile Stirn und Ränder, Wulst- und Stauchformen konkav zur Fließrichtung) auf, welche eine morphologische Vergleichbarkeit mit Lavaströmen zulässt. Reliktische Blockgletscher weißen eine ähnliche Oberflächenformung auf, sind jedoch eisfrei und zum Teil bewachsen. Blockgletscher entstehen aus mächtigen Sedimentakkumulationen (Moränenmaterial und/oder Hangschutt) in welchen Permafrostbedingungen über einen längeren Zeitraum vorherrschen (Barsch 1996). Oberflächenstruktur, Form sowie räumliche Ausdehnung sind das kumulative Ergebnis ihrer gesamten Bildungszeit und dadurch ihrer klimatisch-gesteuerten Vergangenheit. Blockgletscher treten in den österreichischen Alpen häufig auf. Lieb (1996) kartierte flächendeckend für Zentral- und Ostösterreich 1451 Blockgletscher, wovon 1169 aufgrund ihres Erscheinungsbildes als reliktisch klassifiziert wurden (siehe Abb. 1A). Datierungen mit Bezug zur Entstehungsgeschichte können wertvolle Hinweise über das Paläoklima geben. Eine präzise Datierung von Blockgletschern ist schwierig und kann am besten durch einen kombinierten Ansatz von relativen (z.B. Schmidt-Hammer Methode) und absoluten Datierungsmethoden (z.B. Expositionsdatierung) erreicht werden (vgl. Haeberli et al. 2003). In dieser Studie wurde die Schmidt-Hammer Methode zur relativen Oberflächendatierung dreier Blockgletscher im Dösener Tal, Ankogelgruppe angewendet und mit dem absoluten 
Alter benachbarter Moränenablagerungen verglichen. Datierungsergebnisse sowie das Potential der Schmidt-Hammer Methode für die Blockgletscherdateriung werden diskutiert.

\section{Arbeitsgebiet Dösener Tal}

Das Arbeitsgebiet umfasst den hinteren Bereich des glazial geprägten, trogförmigen und Ost-West ausgerichteten Dösener Tales (46 $\left.59^{\circ} \mathrm{N}, 13^{\circ} 26^{\prime} \mathrm{E}, \mathrm{Abb} .1 \mathrm{~A}\right)$, Ankogelgruppe, Hohe Tauern, und umfasst darin die Höhenzone zwischen ca. $2250 \mathrm{~m}$ und $2650 \mathrm{~m}$. Geologisch liegt das Arbeitsgebiet in der Zentralgneiszone des Hochalmkernes mit vorherrschenden nach Westen einfallenden Biotitaugengneisen (Lieb 1996). Dieser Teil des Dösener Tals ist landschaftlich durch vier Nord bis West ausgerichtete Blockgletscher, einem Karsee, markanten Felswänden beidseits der Talachse, sowie einer Karschwelle, welche zum Teil von einem grobblockigen Endmoränenwall überlagert ist, bestimmt (Abb. 1B und 2). Überragt wird das Dösener Tal vom 3086 m hohen, pyramidenartigen Säuleck. Der Endmoränenwall wurde wahrscheinlich während des Egesen-Vorstoßes im ausgehenden Spätglazial abgelagert (Lieb 1996) und ist somit der früheren Jüngeren Dryas (Grönland Stadial 1) vor ca. 12.300-12.400 Jahren vor heute zuzuordnen (Kerschner \& Ivy-Ochs 2008). Drei der vier Blockgletscher (DOE-A, B and C in Abb. 1B und 2) gelten als aktiv, einer (DOE-D in Abb. 1B und 2A) als klimatisch inaktiv (d.h. enthält noch Permafrost bewegt sich jedoch nicht mehr) (Lieb 1996). In dieser Studie lag der räumliche Fokus auf den beiden aktiven Blockgletscher DOE-A (Fläche 0,19 $\mathrm{km}^{2}$ ) und DOE$\mathrm{C}\left(0,007 \mathrm{~km}^{2}\right)$ sowie auf den inaktiven Blockgletscher DOE-D $\left(0,17 \mathrm{~km}^{2}\right)$. Frühere Arbeiten zum Thema Permafrost und Blockgletscher (inklusive Blockgletscherdynamik) fokussierten im speziellen auf den Blockgletscher DOE-A (siehe u.a. Lieb 1996 oder Kaufmann et al. 2007).

\section{Methodik und Lage der Messpunkte}

Der Schmidt-Hammer ist ein leichtes, tragbares Instrument welches Anfang der 1950er Jahre in der Baubranche für Betonhärtetests entwickelt wurde (Schmidt 1950). Dabei wird ein Rückprallwert (Rebound oder R-Wert) eines durch eine Feder gespannten Schlagbolzens, welcher auf eine horizontale Oberfläche einschlägt, gemessen. In geomorphologischen Fragestellungen wird der Schmidt-Hammer verwendet, um die Härte von Gesteinsoberflächen (Felsen, Moränenablagerungen, 


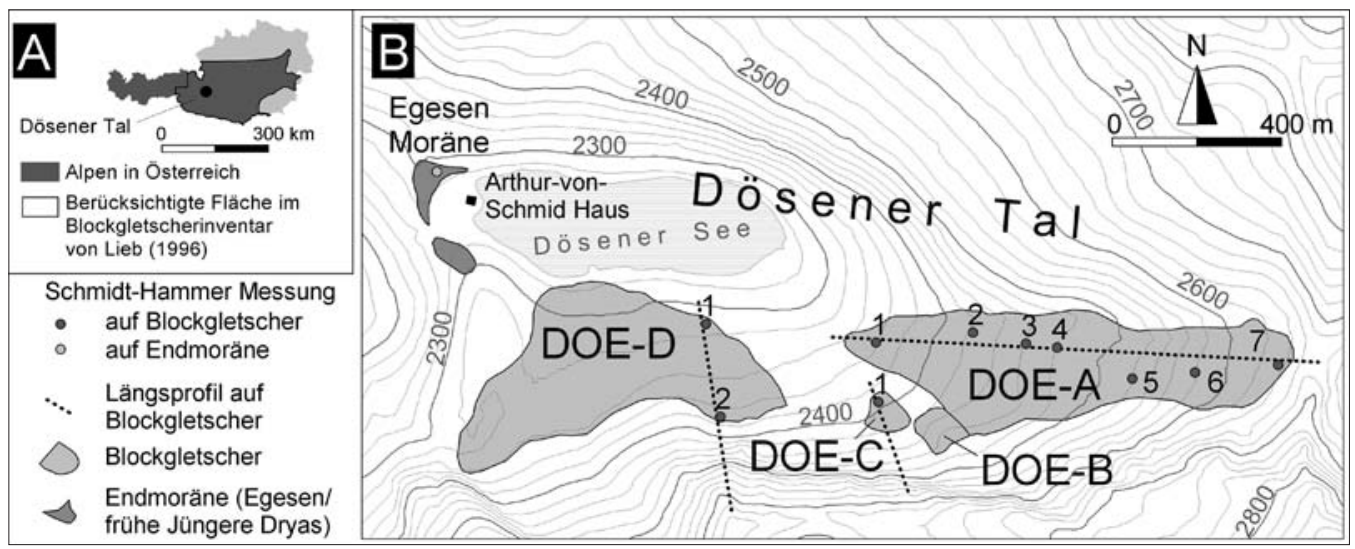

Abb. 1:Das Arbeitsgebiet im Dösener Tal: (A) Überblickskarte sowie räumliche Ausdebnung des 1451 Blockgletscher umfassenden Inventars der zentralen und östlichen österreichischen Alpen nach Lieb (1996); (B) Detailkarte des Arbeitsgebietes mit Lage der vier im Text erwähnten Blockgletscher sowie der Egesen-zeitlichen Endmoräne nabe dem Arthur-von-Schmid Haus. Lagepunke und Codierungen der Schmidt-Hammer Messungen (je 50 gültige Einzelmessungen) auf den Blockgletschern (als Längsprofil ausgerichtete bei DOE-A und DOE-D) sowie auf der Endmoräne sind verortet.

Fig. 1: Study area Dösen Valley: (A) overview map as well as spatial extent of the rock glacier inventory in the central and eastern Alps in Austria by Lieb (1996) including 1451 rock glaciers; (B) detailed map showing the four rock glaciers mentioned in the text and the Egesen (Younger Dryas) terminal moraine close to the Arthur-von-Schmid hut. Locations and codes of the Schmidt-hammer measurements are indicated at the rock glaciers and the terminal moraine. The measurement points at rock glaciers DOE-A and DOE-D are arranged along longitudinal profiles. At each location 50 valid individual Schmid-hammer measurements were carried out.

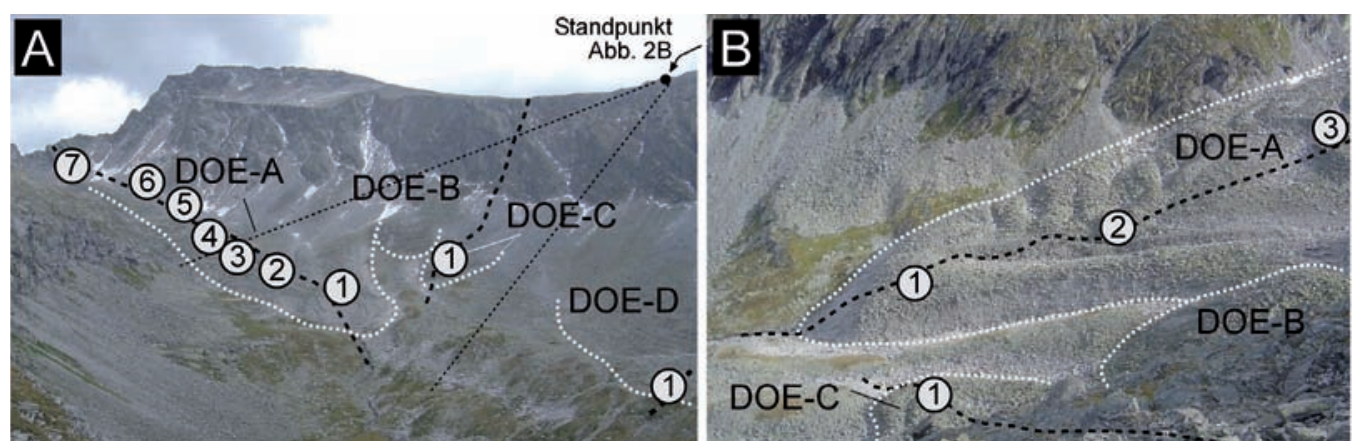

Abb. 2: Das hintere Dösener Tal mit seinen vier Blockgletschern: (A) Blick nach SO, (B) Blick nach N. Zu beachten die großflächige Felswand südlich der Blockgletscher in (A), welche als dominantes Schuttliefergebiet fungiert, die perennierenden Schneeflecken sowie die Wulst-und Stauchformen in (B). Längsprofile sowie Lagepunkte der SchmidtHammer Messungen sind eingezeichnet (Aufnabmedatum: (A) 2.9.2006, (B) 3.9.2006).

Fig.2: The inner Dösen Valley and its four rock glaciers: (A) view towards SE, (B) view towards N. The extensive rock face south of the rock glaciers in (A) is the major source for debris. The small snow patches in (A) are perennial. Numbers indicate Schmidt-hammer measurement sites. (Photographs taken on: (A) 2.9.2006, (B) 3.9.2006). 
etc.) zu testen und dadurch das Grad der Verwitterung und folglich Alter der Gesteinsoberfläche zu bestimmen. Der gemessene R-Wert ist proportional zur Druckfestigkeit der Gesteinsoberfläche und gibt ein relatives Maß der Härte einer Gesteinsoberfläche und damit der Zeit seit Beginn sowie Grad der Verwitterung dieser Oberfläche wieder. Hohe Werte deuten auf ein junges Alter, niedrige Werte hingegen auf ein hohes Alter hin. Die absoluten R-Werte werden aber auch von der Mineralzusammensetzung bzw. der Gesteinsart beeinflusst weshalb nur Oberflächen gleichen Gesteins verglichen werden sollen. Seit Beginn der 1980er Jahre wird diese Methode erfolgreich für glaziale und periglaziale Fragestellungen eingesetzt (z.B. Matthews \& Shakesby 1984, McCarroll 1989). Erst seit wenigen Jahren setzt man den Schmidt-Hammer auch für die Datierung von Blockgletschern ein (z.B. Aoyama 2005, Frauenfelder et al. 2005, Kellerer-Pirklbauer et al. 2007).

In dieser Studie wurde ein analoger Schmidt-Hammer (L-Typ) der Firma Controls, Italien, an 11 Messpunkten (10 auf Blockgletscher, 1 auf Moränenwall) verwendet. Auf jeden der drei untersuchten Blockgletscher wurde an 1 bis 7 Punkten SchmidtHammer Messungen durchgeführt. Bei DOE-A (7 Punkte) und DOE-D (2 Messpunkte) lagen diese Messpunkte entlang eines Längsprofils zwischen der Wurzelzone (oberes Ende) und der Stirn des Blockgletschers (unteres Ende) (Abb. 1B und 2). Die einzelnen Messpunkte wurden so kleinräumig als möglich gehalten $(<10$ $\mathrm{m}^{2}$ ) und lediglich auf Vollformen (z.B. Rücken) durchgeführt, um den Einfluss von lange liegenbleibenden Schneeflecken auf die Verwitterung zu reduzieren. Eine ergänzende Messung erfolgte auf der Egesen-zeitlichen Moräne. Sämtliche Blöcke, an denen Schmidt-Hammer Messungen durchgeführt wurden, waren stabil, wackelten nicht während der Messung und wurden auf möglichst gleiche Gesteinsart ausgewählt.

Die Gesteinsoberflächen selbst waren möglichst horizontal, flach, trocken, sauber und frei von Flechten, sichtbaren Rissen und Klüften (McCarroll 1989, Haeberli et al. 2003, Shakesby et al. 2006). Das arithmetische Mittel von 50 R-Werten eines Messpunktes (vier Schmidt-Hammer Messungen pro Block; nur die beiden mittleren Werte wurden in eine Liste eingetragen; in Summe somit 25 Blöcke pro Messpunkt) sowie das 95\%-Konfidenzintervall wurde für alle 11 Messpunkte ermittelt (Matthews \& Shakesby 1984, Shakesby et al. 2006). Das arithmetische Mittel gibt Auskunft über das Alter der Gesteinsoberfläche und das 95\%-Konfidenzintervall ist ein Indikator für statistisch signifikante Altersunterschiede zwischen zwei Messpunkten. 

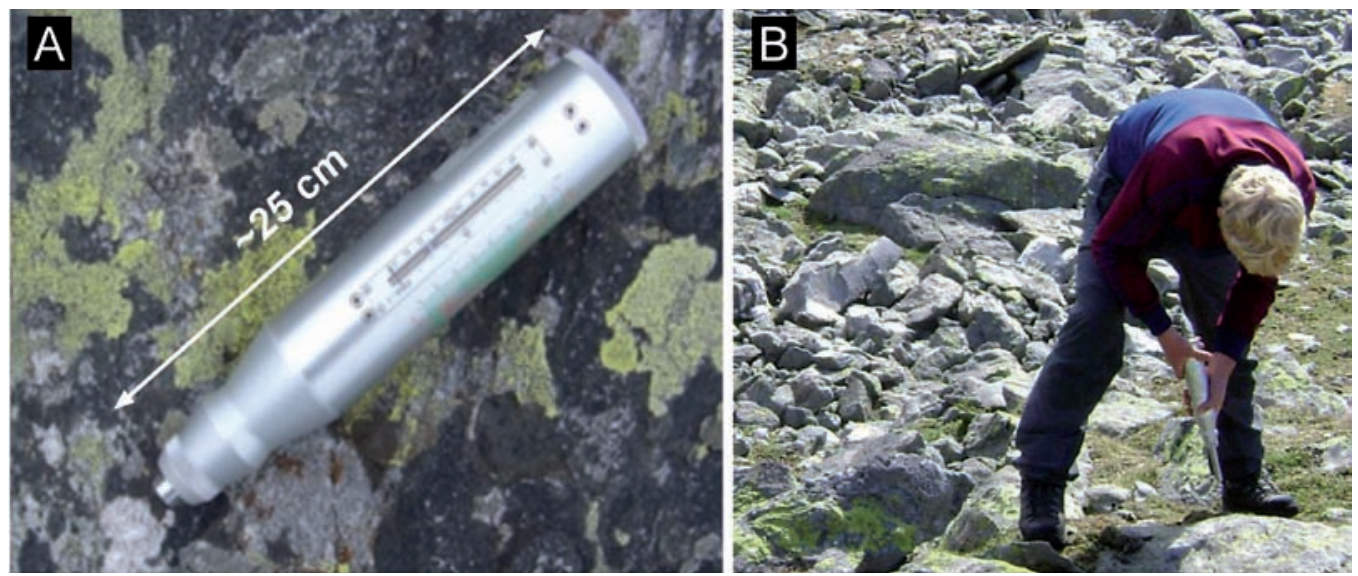

Abb. 3: (A) Der verwendete analoge L-Typ Schmidt-Hammer der Firma Controls, Italien und (B) dessen Einsatz. im Gelände auf einer horizontalen Oberfläche (Aufnahmedatum: (A) 3.9.2007, (B) 24.9.2007).

Fig. 3: (A) The L-type Schmidt-hammer as supplied by the company Controls, Italy, which was used in this investigation. (B) Application of the Schmidt-hammer on a horizontal surface. (Photographs: (A) 3.9.2007, (B) 24.9.2007).

\section{Ergebnisse der Schmidt-Hammer Messungen}

Die Ergebnisse der Schmidt-Hammer Messungen sind in Abbildung 4 sowie Tabelle 1 zusammenfassend dargestellt. An allen 11 Messpunkten sind die 50 Einzelmessungen normal verteilt (d.h. keine bimodale Verteilung) und die Schiefe aller Verteilungen ist relativ gering. Eine Verteilung nach links (negative Schiefe) gibt an, dass der wahre mittlere R-Wert geringer sein kann als der gemessene (also Hinweis auf ein höheres Alter der Felsoberfläche als das tatsächlich gemessene), wohingegen eine Verteilung nach rechts (positive Schiefe) angibt, dass der wahre mittlere R-Wert höher sein kann als der gemessene (also ein geringeres Alter der Felsoberfläche möglich ist). Dies bedeutet, dass auf Basis der Verteilung und Schiefe davon ausgegangen werden kann, dass die 50 Einzelmessungen sehr gut das wahre Alter der Gesteinsoberfläche wiedergeben.

Das arithmetische Mittel der 50 R-Werte aller 11 Messpunkte liegt zwischen 29,7 an der Egesen-Moräne und 47,7 in der Wurzelzone des Blockgletschers DOE-A (Punkt 7) und umfasst damit eine R-Wert Spanne von 18,0. Die 95\%-Konfidenzintervalle sind im Allgemeinen unter $\pm 1,00$ wodurch sich signifikante Altersunterschiede für viele Messpunkte zueinander ergeben. Auffallend ist, dass für die Blockgletscher DOE-A und DOE-D eine statistisch signifikante Abnahme der R-Werte 
von der Blockgletscherfront zum oberen Ende - also zur Wurzelzone - zu erkennen ist. Diese Abnahme ist für die 7 Messpunkte auf DOE-A stetig und umfasst dabei eine Spanne von 12,3 auf der R-Werte Skala. Auf den inaktiven Blockgletscher DOE-D umfasst diese Abnahme immerhin noch 4,5 R-Werte. Auffallend niedrig ist der mittlere R-Wert an der Egesen-Moräne (Unterschied von mindestens 3,6 in R-Werten), was auf ein deutlich höheres Alter im Vergleich zu allen anderen gemessenen Oberflächen schließen lässt.

\section{Altersabschätzung der Blockgletscher}

Wie aus der einschlägigen Schmidt-Hammer Literatur hervor geht, ist die Abnahme von gemessenen R-Werten über die Zeit sehr heterogen und stark von klimatischen wie geologischen Gegebenheiten bestimmt. Trotzdem kann festgestellt werden, dass bei einem $R$-Wertunterschied von $>10$ ein Altersunterschied von mehreren Tausend bis über Zehntausend Jahre angenommen werden kann (z.B. Aa \& Sjåstad 2000, Aoyama 2005, Frauenfelder et al. 2005, Kellerer-Pirklbauer et al. 2007).

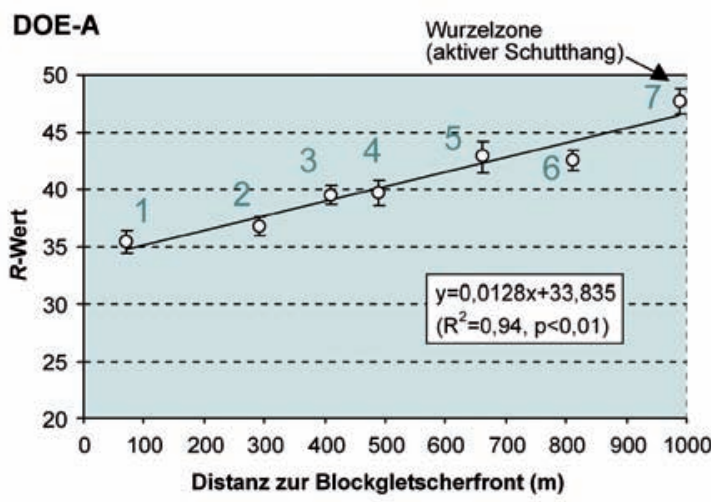

Blockgletscher

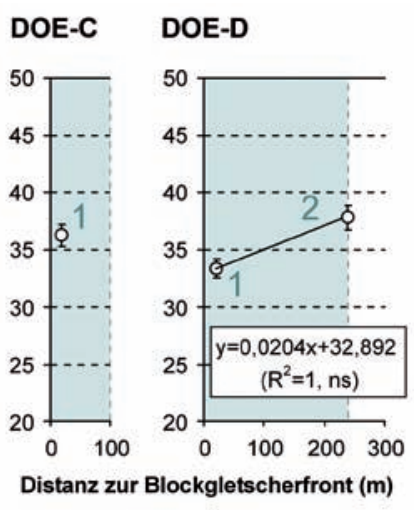

Egesen Moräne

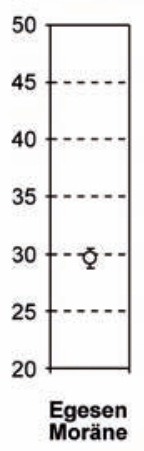

Abb. 4: Ergebnisse der Schmidt-Hammer Messungen an den 11 Messpunkten im Dösener Tal: Die R-Werte zeigen die arithmetischen Mittelwerte sowie das 95\%-Konfidenzintervall an allen Messpunkten. Bei den drei Blockgletschern DOE-A, DOE-C und DOE-D sind die Ergebnisse der einzelnen Messpunkte in Abhängigkeit vom Abstand zum Blockgletscherende dargestellt. Für DOE-A und DOE-D wurde auch die Ausgleichsgerade ermittelt (für beide sehr äbnlich), wobei diese lediglich für DOE-A als statistisch signifikant einzustufen ist. Abkürrungen: $\mathrm{R}^{2}=$ Bestimmtheitsmaß oder Determinationskoeffizient, $p=$ Signifikanz, ns=nicht signifikant.

Fig. 4: Results of the Schmidt-hammer measurements from 11 points in the Dösen Valley: R-values are given as arithmetic means of 50 individual readings (4 impacts per boulder; only the two middle values were noted) with $95 \%$ confidence interval. $\mathrm{R}$-values are plotted against the distance from the terminus at the two rock glaciers DOE-A and DOE-D. The regression line is significant for DOE-A only $(\mathrm{p}<0.01)$. 
Klimawandel in Österreich

Tabelle 1: Zusammengefasste Ergebnisse der Schmidt-HammerMessungen an den 11 Messpunkten im Dösener Tal.

Table 1: Summarised results of the Schmidt-hammer measurements at the 11 points in the Dösen Valley.

\begin{tabular}{lccc}
\hline Landform & Standort & $\begin{array}{c}\text { Mittleres } R \\
\pm 95 \% \text { CI }(n=50)\end{array}$ & $\begin{array}{c}\text { Schiefe der } \\
\text { Verteilung }\end{array}$ \\
\hline Blockgletscher DOE-A & DOE-A1 & $35,4 \pm 1,00$ & $-0,26$ \\
& DOE-A2 & $36,8 \pm 0,83$ & $-0,04$ \\
& DOE-A3 & $39,5 \pm 0,84$ & 0,56 \\
& DOE-A4 & $39,7 \pm 1,11$ & 0,47 \\
& DOE-A5 & $42,8 \pm 1,36$ & 0,01 \\
& DOE-A6 & $42,5 \pm 0,86$ & 0,70 \\
Blockgletscher DOE-C & DOE-A7 & $47,7 \pm 1,14$ & $-0,23$ \\
Blockgletscher DOE-D & DOE-C1 & $36,2 \pm 0,94$ & $-0,18$ \\
& DOE-D1 & $33,3 \pm 0,80$ & $-0,33$ \\
Egesen-Moräne & DOE-D2 & $37,8 \pm 1,05$ & $-0,83$ \\
\hline
\end{tabular}

Die untersuchten Blockgletscher im Dösener Tal haben sich erst nach erfolgtem Rückzug des späteiszeitlichen Dösener Gletschers in höhere Lagen bilden können. Wie die kartierten Egesen-zeitlichen Moränen im Nahbereich des Arthur-vonSchmid Hauses zeigen, kann die Entwicklung der untersuchten Blockgletscher somit nur nach dem Egesen-Vorstoß im ausgehenden Spätglazial begonnen haben. Wie absolute Datierungen von Egesen-zeitlichen Moränen in den westlichen österreichischen Alpen zeigen, erfolgte die Stabilisierung dieser Moränen in der früheren Jüngeren Dryas vor ca. 12.300-12.400 Jahren vor heute (absolut datiert mit Hilfe der Expositionsdatierung von kosmogen in situ produzierten Radionukliden ${ }^{10} \mathrm{Be}$ durch Kerschner \& Ivy-Ochs 2008). Dies bedeutet, dass der niedrigste R-Mittelwert im Dösener Tal von 29,7 an der Egesen-Moräne einem Alter von ca. 12.350 Jahren entspricht. Für den höchsten gemessenen mittleren $R$-Wert von 47,7, welcher am hoch aktiven Schutthang in der Wurzelzone des Blockgletschers DOE-A gemessen wurde, kann ein Alter von null bzw. wenigen Jahren angenommen werden. Erwähnenswert ist, dass zwischen dem Moment, in dem sich ein Riss in einer Felswand bildet (und somit die Verwitterung einer Gesteinsoberfläche beginnt) und dem Steinschlagereignis (Transport des Blocks von der Felswand in die Wurzelzone des Blockgletschers) sehr viele Jahre bis Jahrhunderte vergehen können. Eine Quantifizierung dieser Zeitspanne ist daher schwierig. 
Geht man von einem linearen Zusammenhang zwischen R-Wert und Zeit aus (siehe dazu auch die diesbezügliche Diskussion in Skakesby et al. 2006), so kann eine Alterkalibrierung der erzielten R-Werte und somit eine absolute Datierung der Gesteinsoberflächen durchgeführt werden. Wie das Ergebnis dieser Alterskalibrierung zeigt (Abb. 5), ergibt sich für das hintere Dösener Tal eine mittlere Abnahme des R-Wertes von 1,46 in 1000 Jahren. Nach einem einfachen Ansatz von Skakesby et al. (2006) können auch die Fehlergrenzen dieser Alterskalibrierung quantifiziert werden. Entsprechend dieses Ansatzes werden für die Egesen-Moräne als auch für den Messpunkt in der Wurzelzone des Blockgletschers DOE-A die Fehlergrenzen einzeln durch die entsprechenden 95\%-Konfidenzintervalle berechnet und als zweiten Schritt mit einer geraden Linie verbunden. Die Fehlergrenze für die EgesenMoräne beträgt \pm 607 Jahre, jene der Wurzelzone von DOE-A (Punkt 7) \pm 774 Jahre. Folglich muss von einer Altersunsicherheit von rund \pm 700 Jahre an jedem SchmidtHammer Messpunkt im Dösener Tal ausgegangen werden.

Wie man weiter aus Abbildung 5 entnehmen kann, ergibt sich für alle 10 Messpunkte auf den drei Blockgletschern ein Alter von unter 10.000 Jahren. Das höchste Alter auf einem Blockgletscher wurde für die Stirn des Blockgletschers DOE-D mit 9860 Jahre ermittelt. Die Stirn der Blockgletscher DOE-A und DOE-C sind mit $\sim 8430$ bzw. $\sim 7910$ Jahre etwas jünger. Alle drei Blockgletscher sind somit seit vielen tausend Jahren wesentliche Landschaftselemente des hinteren Dösener Tales. Für den Messpunkt in der Wurzelzone des inaktiven Blockgletschers DOE-D (Punkt 2) ergibt sich ein Alter von 6780 Jahre, was darauf hindeutet, dass in dieser Zeit die Ernährung des Blockgletschers durch Schutt aus den dahinter liegenden Felswänden markant abnahm. Aufgrund der tieferen Lage dieses Blockgletschers scheint dies auch möglich. Anders ist die Situation beim großen aktiven Blockgletscher DOE-A. Die Ergebnisse der sieben Messpunkte auf DOE-A weißen auf eine kontinuierliche Entwicklung dieser noch heute aktiven Landschaftsform in den letzten zumindest 8.400 Jahren hin. Diese Feststellung impliziert, dass die klimatischen Bedingungen im Dösener Tal während dieses gesamten Zeitraumes ab einer Seehöhe von rund $2350 \mathrm{~m}$ auf West und Nord exponierten Hängen mit Grobschuttauflage permafrostgünstig waren. Ebenso kann eine alleinige Entstehung von DOE-A seit dem Ende der so genannten „Kleinen Eiszeit“ (d.h. seit 1850 AD) - wie für verschiedene Blockgletscher in den Ötztaler Alpen (z.B. Berger et al. 2004 und zitierte Literatur darin) vermutet - ausgeschlossen werden.

Quantitative Oberflächenbewegungsraten für den Blockgletscher DOE-A wurden für den Zeitraum 1954-2005 durch Kaufmann et al. (2007) mit Hilfe von photogrammetrischen und geodätischen Methoden ermittelt. Entsprechend dieser Arbeit schwankte die mittlere jährliche Oberflächenbewegungsrate in diesem Zeitraum 

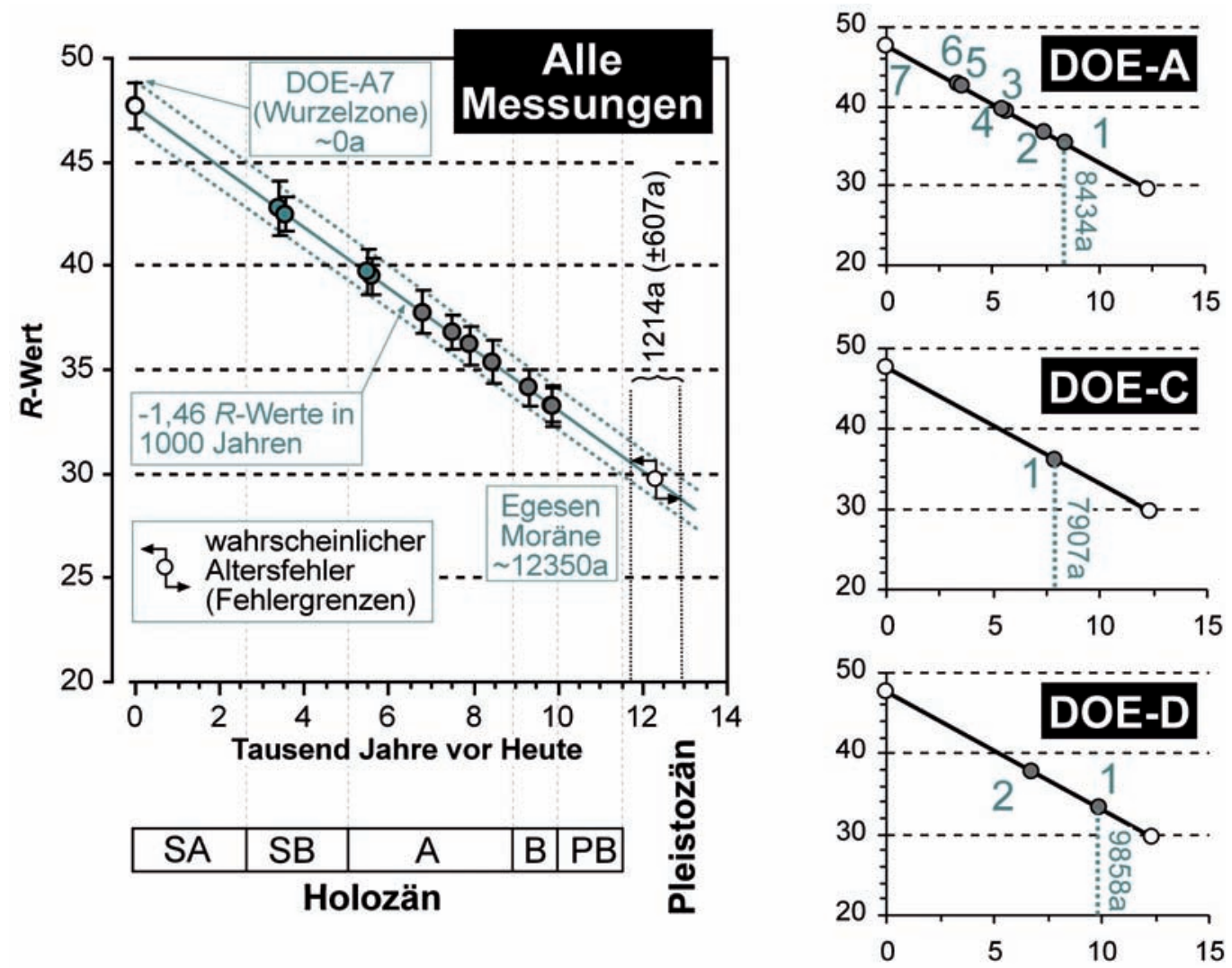

Abb. 5: Absolute Altersabschätzung der Schmidt-Hammer Messergebnisse im Dösener Tal für alle 11 Messungen gemeinsam sowie getrennt für die drei untersuchten Blockgletscher DOE- A, DOE-C und DOE-D. Fürden Messpunk.t an der Egesen-Moräne (=ältester Messpunkt) wird auf Basis von absoluten Datierungen in Westösterreich (Kerschner \& Ivy-Ochs 2008) ein Alter von 12.350 Jabre angenommen. Für den Messpunkt in der aktiven Wurzelzone des Blockgletschers DOE-A (=jüngster Messpunkt) ergibt sich ein Alter von O Jabre. Diese beiden Messpunkte sind als offene Kreise dargestellt und mit einer geraden Linie verbunden. Auf Basis dieser Linie ergeben sich die Alter der anderen 9 Messpunkte dargestellt als graue Kreise. Die strichlierten Linien beidseits der durchgezogenen Linie geben die Feblergrenze wieder, welche im Bereich von \pm 700 Jahre liegt (Details siehe Text). Abkü̈rzungen: a=Jahr; Chronozonen im Holozän (in kalibrierten Jabren nach Haas et al. 1998): SA=Subatlantikum, SB=Suboreal, $A=$ Atlantikum, $B=$ Boreal und $P B=$ Preboreal.

Fig. 5: Absolute age calibration of the Schmidt-hammer measurements in the Dösen Valley for all 11 measurement points together and separately for the three rock glaciers DOE-A, DOE-C and DOED. The point at the Egesen terminal moraine (oldest site) has an assumed age of 12.350 years based on absolute dates from western Austria (cf. Kerschner \& Ivy-Ochs 2008). For the uppermost point of DOE-A (the youngest part of the rock glacier) an age of $\sim 0$ is assumed. Both values are shown as open circles and are connected with a line. Using this line the ages of the 9 points in between (grey circles) are obtained. The dashed lines on either side of the solid line indicate the estimated error which is in the range \pm 700 years. 
zwischen 13,4-37,4 cm. Nimmt man nun diese Jahresbewegungsbeträge als konstant über die Zeit (was jedoch sehr fraglich ist!) und kombiniert man diese mit der Länge des Blockgletschers DOE-A, so ergibt sich ein Alter von 2.700 bis 7500 Jahre. Folglich ist die Größenordnung der beiden Altersabschätzungsansätze - SchmidtHammer und Photogrammetrie/Geodäsie - zumindest grob vergleichbar.

$\mathrm{Zu}$ allerletzt sollte aber nicht vergessen werden, dass das Gesamtalter eines Blockgletschers wesentlich älter sein kann als das Alter der Blockgletscheroberfläche selbst, da sich ein Blockgletscher ähnlich einem Förderband zu Tal bewegt. Blöcke an der Oberfläche eines aktiven Blockgletschers stürzen an den Fuß der Blockgletscherstirn und werden von der vorrückenden Eis- und Schuttmasse überschüttet (vgl. Haeberli et al. 2003). Deshalb ergeben Altersdatierungen von Blockgletscheroberflächen lediglich ein Mindestalter eines Blockgletschers (vgl. Frauenfelder et al. 2005).

\section{Fazit}

Wie diese Studie im Dösener Tal zeigt, ist die Anwendung des Schmidt-Hammers eine einfache Methode um das relative Alter von alpinen Blockgletschern abzuschätzen. Besonders bei großen Blockgletschern, wo an mehreren Punkten entlang eines Längsprofils Schmidt-Hammer Messungen durchgeführt werden können, ermöglicht diese Methode die Erstellung von detaillierten relativen Chronologien. Mit ergänzenden Altersangaben ermittelt durch absolute Datierungsmethoden (vgl. Haeberli et al. 2003) lassen sich die gemessenen Schmidt-Hammer Werte kalibrieren und in absolute Alter umwandeln. Somit können verlässliche Chronologien über die Blockgletschergenese ermittelt werden. Ein großer Nachteil der SchmidtHammer Methode ist die relativ grobe zeitliche Auflösung. Zeitliche Unsicherheiten von einigen hundert bis über tausend Jahre müssen in Kauf genommen werden, die hier vorgestellten Datierungen haben also eine erheblich Schwankungsbreite. Nichtsdestoweniger zeigen meine Ergebnisse sehr klar, dass es sich bei den Blockgletschern im Dösener Tal um alte Landschaftsformen handelt, welche in ihrer heutigen Form über einen Großteil des Holozäns gebildet worden sind.

\section{Danksagung}

Diese Studie wurde im Rahmen des Projektes ALPCHANGE (www.alpchange. at) durchgeführt, welches durch den Fonds zur Förderung der wissenschaftlichen Forschung (FWF) als Projekt Nr. P-18304-N10 finanziell unterstützt wird. 


\section{Klimawandel in Österreich}

\section{Literatur}

Aa R. \& Sjåstad J.A., 2000: Schmidt hammer age evaluation of the moraine sequence in front of Bøyabreen, western Norway. Norsk Geologisk Tidsskrift 80: 27-32.

Aoyama M., 2005: Rock glaciers in the northern Japanese Alps: palaeoenvironmental implications since the Late Glacial. Journal of Quaternary Science 20: 471-484.

Barsch D., 1996: Rock Glaciers: Indicators for the Present and Former Geoecology in High Mountain Environments. Springer Series in Physical Environment 16, Springer Verlag, Berlin, 331 S.

Berger J., Krainer K. \& Mostler W., 2004: Dynamics of an active rock glacier (Ötztal Alps, Austria). Quaternary Research 62: 233-242.

Frauenfelder R., Laustela M. \& Kääb A., 2005: Relative dating of Alpine rockglacier surfaces. Zeitschrift für Geomorphologie 49: 145-166.

Haas J.N, Richoz I., Tinner W. \& Wick L., 1998: Synchronous Holocene climatic oscillations recorded on the Swiss Plateau and at timberline in the Alps. The Holocene 8: 301-309.

Haeberli W., Brandovà D., Burga C., Egli M., Frauenfelder R., Kääb A. \& Maisch M., 2003: Methods for absolute and relative age dating of rock-glacier surface in alpine permafrost. Proceedings of the Eighth International Conference on Permafrost, Zurich, Switzerland, July 21-25, 2003: 343-348.

Kaufmann V., Ladstädter R. \& Kienast G., 2007: 10 years of monitoring of the Doesen rock glacier (Ankogel group, Austria) - A review of the research activities for the time period 1995-2005. Proc. of the Fifth Mountain Cartography Workshop, Bohinj, Slovenia, March 29-April 1, 2006: 129-144.

Kellerer-Pirklbauer A., 2008: The Schmidt-hammer as a Relative Age Dating Tool for Rock Glacier Surfaces: Examples from Northern and Central Europe. Proceedings of the Ninth International Conference on Permafrost, Fairbanks, Alaska, June 29-July 3, 2008: (in Druck).

Kellerer-Pirklbauer A., Wangensteen B., Farbrot H. \& Etzelmüller B., 2007: Relative surface agedating of rock glacier systems near Hólar in Hjaltadalur, Northern Iceland. Journal of Quaternary Science 23: 137-151.

Kerschner H. \& Ivy-Ochs S., 2008: Palaeoclimate from glaciers: Examples from the Eastern Alps during the Alpine Lateglacial and early Holocene. Global and Planetary Change 60 (1-2): 58-71.

Lieb G.K., 1996: Permafrost und Blockgletscher in den östlichen österreichischen Alpen. Arbeiten aus dem Institut für Geographie, Universität Graz 33: 9-125.

Matthews J.A. \& Shakesby R.A., 1984: The status of the 'Little Ice Age' in southern Norway: relativedating of Neoglacial moraines with Schmidt hammer and lichenometry. Boreas 13: 333-346.

McCarroll D., 1989: Potential and limitations of the Schmidt hammer for relative dating, Field tests on Neoglacial moraines, Jotunheimen, southern Norway. Arctic and Alpine Research 21: 268-275.

Schmidt E., 1950: Der Beton-Prüfhammer - Ein Gerät zur Bestimmung der Qualität des Betons im Bauwerk. Schweizerische Bauzeitung 68: 378-379.

Shakesby R.A., Matthews J.A. \& Owen G., 2006: The Schmidt hammer as a relative-age dating tool and its potential for calibrated-age dating in Holocene glaciated environments. Quaternary Science Reviews 25: 2846-2867. 


\title{
Prähistorische Besiedlung von zentralen Alpentälern in Bezug zur Klimaentwicklung
}

\author{
Klaus Oeggl ${ }^{1)}$ \& Kurt Nicolussi ${ }^{2)}$ \\ 1) Institut für Botanik der Universität Innsbruck, Innsbruck \\ 2) Institut für Geographie der Universität Innsbruck, Innsbruck
}

\section{Abstract}

\section{Prehistoric Colonization of Central Alpine Valleys Related to Climate Development}

Climate impact on ecosystems is a matter of thresholds and scale. Here we present the compilation of the pre-historic colonization of central Alpine valleys tracked by pollen analyses which we correlate with the record of sub-fossil logs from altitudes above $2000 \mathrm{~m}$, as well as with the oxygen-isotope curve from Greenland ice cores (NGRIP). Both serve as proxies for the climate development in the Alps, but also on a larger (northern hemispheric) scale. On long-term scales no relationship between settlement and climate was detectable, but shorter time-scales ( $<500$ years) indicated a positive correlation, at least for parts of the Neolithic (e.g. between ca. 4500 and 3000 and ca. 2800 and 2400 BC, before Christ). A negative correlation was indicated for the period from ca. 3000 until 2800 BC. Climate deteriorations (e.g. as around $2700 \mathrm{BC}$ ) seem not to have severely affected the settling in central Alpine valleys indicating robust and resistant agricultural structures. From the Bronze Age onwards there was no significant correlation between climate and peopling of these Alpine valleys. These examples showed that climate has been an important trigger, but the relationship between climate and settling is more complex (e.g. influence of socio-economic structures, ore resources, etc.).

\section{Einleitung}

Der Einfluss des Klimas auf die Siedlungstätigkeit ist mannigfaltig. Er kann zwischen Extremen, wie anhaltende Trocken- bis Dürrezeiten, die zum Zerfall von Kulturen führten (deMendocal 2001, Haug et al. 2003), bis hin zu Niederschlagsreichtum schwanken, der ebenfalls Veränderungen im Siedlungsverhalten bedingen kann (Turney und Brown 2007, Tinner et al. 2003, Arbogast et al. 2006). In den Alpen kommt mit dem Relief noch die Abnahme der Temperatur mit der Höhe hinzu, die die Abhängigkeit der Nutzungsstrukturen vom Klima noch weiter akzentuieren (Schmidt et al. 2002, 2007). Daraus könnte man verallgemeinern, dass Klimagunst die Siedlungsaktivität fördert, beziehungsweise umgekehrt Klimaverschlechterungen zu einem Rückgang in der Siedlungstätigkeit führen (Berglund 2002). Im scheinbaren Gegensatz dazu steht jedoch eine Reihe von jüngeren pollenanalytischen Befunden 
aus den Tallagen $(600-1500 \mathrm{~m})$ im zentralen Alpenraum zur Vegetations- und Siedlungsgeschichte während der Bronze- und Eisenzeit (Wahlmüller 2002, Oeggl et al. 2005, Schmidl et al. 2005), die auch für klimatisch ungünstige Phasen eine rege Siedlungsaktivität belegen. Diese Ergebnisse stellen eine rein deterministische Betrachtungsweise der Beziehung zwischen Klima und Siedlungstätigkeit in Frage. Im folgenden Bericht soll dieser Frage für die prähistorische Siedlungstätigkeit (Zeitfenster vom Neolithikum, 4500 v. Chr., bis zum Beginn der Römerzeit) in Tälern

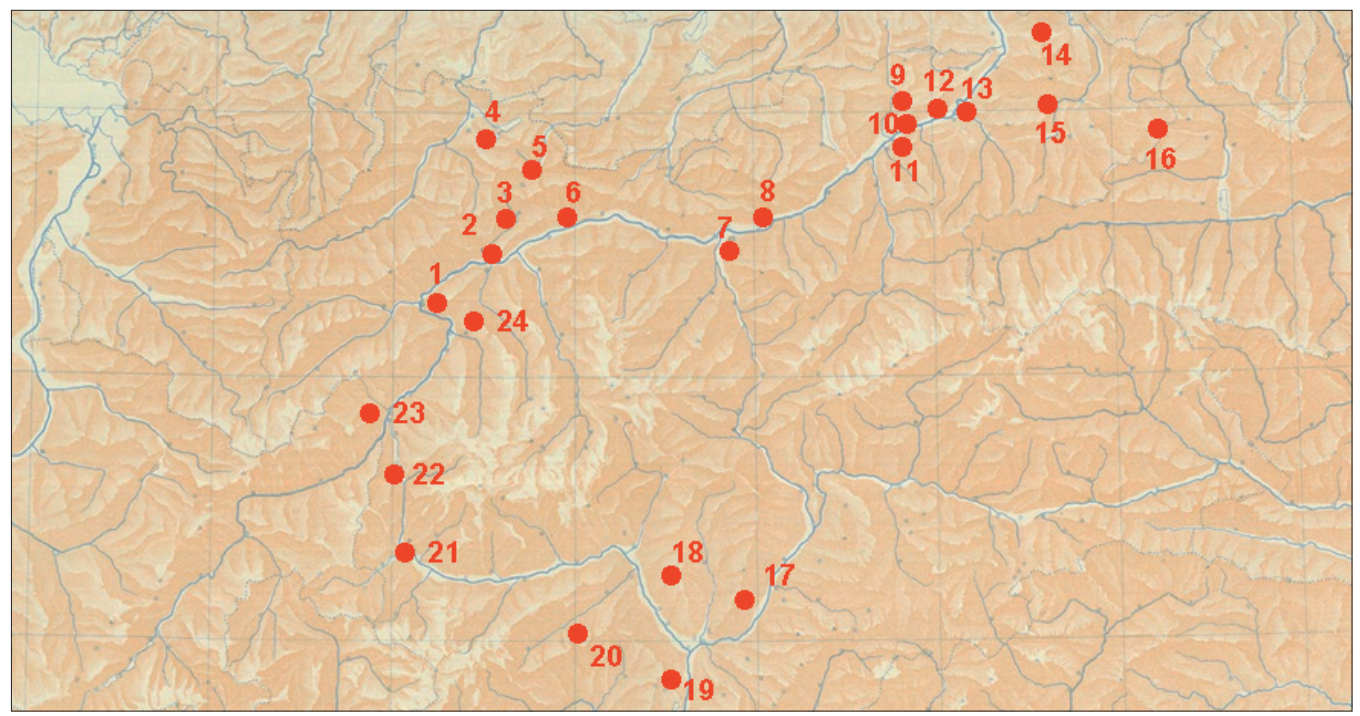

Abb. 1: Lage der pollenanalytisch untersuchten Moore zur Siedlungsentwicklung im mittleren Alpenraum: 1 Plemun Weiher (Walde 2006), 2 Tollinger Moor (Hubmann 1994), 3 Seeeck (Walde, unpubl. Daten), 4 Heiterwanger Moor (Walde, unpubl. Daten), 5 Lermoos (Oeggl 1998), 6 Mieminger See (Wablmüller 1985), 7 Tantegert (Oeggl, unpubl. Daten), 8 Luchner Moor (Oeggl 1999), 9 Brandenberger Moor (Eidenhammer 1999), 10 Frauensee (Walde 1999), 11 Oberkienberg (Walde 1999), 12 Lindenmoos (Oeggl, unpubl. Daten), 13 Kirchbichl (Wablmüller 1985), 14 Schwemm (Oeggl 1988), 15 Giering (Bortenschlager \& Bortenschlager 1984), 16 Fieberbrunn (Eidenhammer 1999), 17 Wallnereck. (Wablmüller-Oeggl, unpubl. Daten), 18 Tammerle Moos (Wablmüller 1990), 19 Montiggler See (Kompatscher \& Oeggl 2005), 20 Totes Moos (Heiss et al. 2005$), 21$ Flora Moos (Walde, unpub. Daten), 22 Fubrmannsloch (Walde, unpubl. Daten), 23 Komperdell (Wablmüller, 2002 ), 24 Piller Sattel (Hubmann, 1994)

Fig. 1: Location of mires with palynological records of human impact and settlement development in the central part of the Alps: 1 Plemun Weiher (Walde 2006), 2 Tollinger Moor (Hubmann 1994), 3 Seeeck (Walde, unpubl. data), 4 Heiterwanger Moor (Walde, unpubl. data), 5 Lermoos (Oeggl 1998), 6 Mieminger See (Wahlmüller 1985), 7 Tantegert (Oeggl, unpubl. data), 8 Luchner Moor (Oeggl 1999), 9 Brandenberger Moor (Eidenhammer 1999), 10 Frauensee (Walde 1999), 11 Oberkienberg (Walde 1999), 12 Lindenmoos (Oeggl, unpubl. data), 13 Kirchbichl (Wahlmüller 1985), 14 Schwemm (Oeggl 1988), 15 Giering (Bortenschlager \& Bortenschlager 1984), 16 Fieberbrunn (Eidenhammer 1999), 17 Wallnereck (Wahlmüller-Oeggl, unpubl. data), 18 Tammerle Moos (Wahlmüller 1990), 19 Montiggler See (Kompatscher \& Oeggl 2005), 20 Totes Moos (Heiss et al. 2005), 21 Flora Moos (Walde, unpub. data), 22 Fuhrmannsloch (Walde, unpubl. data), 23 Komperdell (Wahlmüller, 2002 ), 24 Piller Sattel (Hubmann, 1994) 
der mittleren Alpen nachgegangen werden, wobei pollenanalytische, archäologische und dendrochronologische Ergebnisse miteinander verknüpft werden.

\section{Methodik}

Das bezüglich der prähistorischen Siedlungsaktivität bearbeitete Gebiet umfasst das Nordtiroler Inntal mit seinen Seitentälern und das Etsch- und Eisacktal der Provinz Bozen-Südtirol (Abb. 1). Aus dieser Region existieren zahlreiche Pollendiagramme (Wahlmüller 1993), die neben der holozänen Vegetationsentwicklung auch die Siedlungsgeschichte im jeweiligen Gebiet erfassen. Aus diesen wurden 24 Pollendiagramme für diese Studie nach folgenden Kriterien ausgewählt:

- Die Pollendiagramme müssen von Lokalitäten aus den Tieflagen (600 - 1500 m) stammen.

- Der Durchmesser der untersuchten Lokalität muss kleiner als $300 \mathrm{~m}$ sein, damit überwiegend die lokale Vegetationsentwicklung erfasst wird (cf. Jacobson \& Bradshaw 1981).

- Die ausgezählte Mindestpollensumme muss über 500 Pollenkörner/Probe betragen.

- Die Pollendiagramme müssen mindestens fünf Radiokarbondatierungen aufweisen, um eine ausreichende Tiefen-Zeit-Modellierung zu ermöglichen.

Der Nachweis der Siedlungsaktivität an den einzelnen Lokalitäten erfolgte pollenanalytisch über die sogenannte „Landnahme“ nach Iversen (1941, 1972). Dabei zeigen Einbrüche in den Kurven der Klimaxbaumarten, begleitet von Siedlungsund Kulturzeigern sensu Behre (1981) sowie von Holzkohlepartikeln (Brandrodung), Rodungen von Wäldern zur Gewinnung von Siedlungsflächen an. Derartige Siedlungsphasen wurden für jedes der 24 Pollendiagramme erfasst, und anschließend wurde die Anzahl der Pollendiagramme mit einer Siedlungsphase in 100-Jahr-Intervallen entsprechend den kalibrierten ${ }^{14} \mathrm{C}$-Daten aufgetragen (Abb. 2).

Der Abgleich der Siedlungsaktivität mit der Klimaentwicklung erfolgte sowohl mit einem regionalen als auch einem überregionalen Klimaproxy-Datensatz. Als regionalen Klimazeiger verwendeten wir die Summenkurve aller dendrochronologisch datierten subfossilen Baumstämme, die im mittleren Alpenraum im Bereich zwischen 2000 und $2500 \mathrm{~m}$ N.N. außerhalb von Gletschervorfeldern gefunden wurden. Der klimatische Zusammenhang besteht darin, dass es unter günstigen Klimabedingungen zu einer Verschiebung der alpinen Baumgrenze nach oben und gleichzeitig zu einer Verdichtung der Baumbestände im Waldgrenzökoton kommt (Nicolussi et 
al. 2005). Umgekehrt sterben bei einer Klimaverschlechterung mehr Bäume ab, und synchron dazu erfolgt eine Auflichtung der Bestände mit einem Absinken der Waldund Baumgrenze. Dadurch werden während und am Ende ungünstiger Klimaphasen potenziell mehr Baumleichen abgelagert als in Perioden mit günstigen klimatischen Bedingungen. Zur überregionalen Kontrolle, ob subfossile Bäume tatsächlich ein Signal für das Paläoklima der Alpen liefern, wurde die Baumbelegkurve mit der Sauerstoff-Isotopenkurve $\left(\delta^{18} \mathrm{O}\right)$ von NGRIP (North Greenland Ice Core Project Members 2004) unterlegt (Abb. 2). Über weite Strecken entspricht die Sauerstoffisotopenkurve dem Verlauf der Baumbelegungskurve, sodass letztere tatsächlich als Proxy-Klimadatensatz für die Alpen verwendet werden kann.

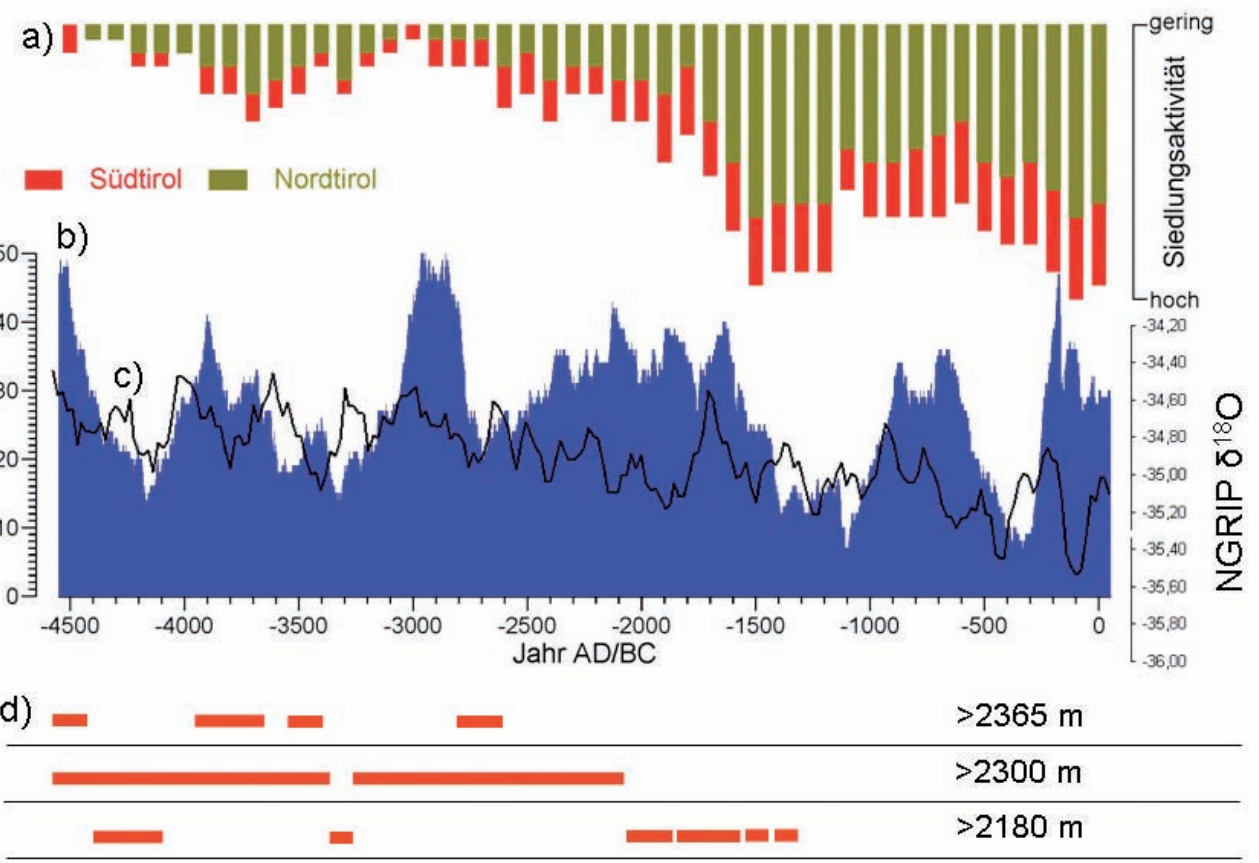

Abb. 2: Die Siedlungs- und Klimaentwicklung im mittleren Alpenraum im Zeitraum zwischen 4500 v. Chr. und Christi Geburt: a) Siedlungsaktivität im mittleren Alpenraum anband der Pollenanalysen an den in Abb. 1 genannten Lokalitäten nach kalibrierten ${ }^{14} C$-Daten, zusätzlich unterteilt für Nord-baw. Südtirol, b) Summenkurve der dendrochronologisch datierten subfossilen Baumstämme aus dem mittleren Alpenraum, c) Sauerstoff-Isotopenkurve $\left(\delta^{18} O\right)$ von NGRIP (North Greenland Ice Core Project Members 2004), 100-Jahr-Mittel, d) Lage der Baumgrenze in den nördlichen Zentralalpen.

Fig. 2: Settlement and climate history of the central parts of the Alps from 4500 BC until birth of Christ: a) colonization of the Alps recorded by pollen analyses from mires shown in fig. $1, b$ ) cumulative curve of dendrologically dated subfossil logs from the central part of the Alps, c) NGRIP $\delta^{18} \mathrm{O}$-values (North Greenland Ice Core Project Members 2004), centennial mean, d) location of the timberline in the northern central Alps. 


\section{Ergebnisse}

In der Mitte des 5. Jahrtausends v. Chr. (Frühe Jungsteinzeit $=$ frühes Neolithikum) wurde offenbar der zentrale Alpenraum für die Menschen auch als Dauersiedlungsraum interessant. Die ersten Siedlungstätigkeiten zeichnen sich in den Pollendiagrammen aus der randalpinen Buchen-Tannen-Fichtenwaldzone und der zwischenalpinen Fichten-Tannenzone für die Zeit um 4500 v. Chr. ab (Abb. 2). Klimatisch steht diese Besiedelungsphase am Ende des mehrphasigen mittelholozänen Wärmeoptimums, das von ca. 7000 - 3500 v. Chr. gedauert hat (Kalis et al. 2003). Während dieser Wärmeperiode erreichte die Waldgrenze in den zentralen Ostalpen Höhen von 2400 bis 2550 m (Pott et al. 1995, Staffler \& Feichter 1999, Krapf 2001, Nicolussi et al. 2005), die im späteren Holozän nie mehr erreicht wurden. Vergleichbare Waldgrenzen für diesen mittelholozänen Zeitraum sind auch für die Westalpen belegt (u. a. Burga und Perret 1998), die mit geringer Ausdehnung der Alpengletscher korrespondieren (u. a. Hormes et al. 2001). Diese früh-neolithische Besiedlungsphase unter überwiegend klimatisch günstigen Bedingungen kulminiert um 3700 v. Chr. Anschließend ist ein Rückgang feststellbar, der um 3000 v. Chr. seinen Tiefststand erreichte. Diese negative Siedlungsentwicklung im 4. Jahrtausend v. Chr. deckt sich zumindest abschnittsweise mit einer Gletschervorstoßphase (Rotmoos II Schwankung), die etwa in den Zeitraum zwischen 3700 und 3200 datiert (Bortenschlager 1970, Jörin et al. 2008). Möglicherweise ist die Rotmoos Schwankung durch stark schwankende Klimabedingungen mit phasenweise kühlen und niederschlagsreichen Verhältnissen charakterisiert. Diese könnte in den neolithischen Pfahlbausiedlungen des Schweizer Alpenvorlandes zu katastrophalen Auswirkungen geführt haben (u.a. Schibler et al. 1997). Entsprechende Untersuchungen aus den Pfahlbausiedlungen am Nordrand der Ostalpen stehen noch aus. Die zumindest phasenweise klimatisch ungünstigen Bedingungen im 4. Jahrtausend v. Chr., wie sie aus den dendrochronologischen Befunden ersichtlich sind (Abb. 2), scheinen jedoch zu keiner gänzlichen Unterbrechung in der Siedlungstätigkeit geführt zu haben. In dieser Periode scheint sogar eine negative Korrelation zwischen Siedlungsintensität und Klima zu bestehen (Abb. 3).

Erst im Spätneolithikum (ab ca. 3000 v. Chr.) nimmt die Siedlungstätigkeit wieder zu, ab der frühen Bronzezeit (ca. 2200 v. Chr.) wurden auch die Seitentäler intensiver besiedelt, und in der mittleren Bronzezeit (ca. 1500 v. Chr.) erreichte die Siedlungstätigkeit ihre maximale Ausdehnung. Mit dem Beginn der Urnenfelderzeit (ca. 1300 v. Chr.) ist in den Pollenprofilen eine leichte Abnahme der Siedlungstätigkeit festzustellen, die bis in die Eisenzeit anhielt. Mit der Latènekultur (ca. 500 v. Chr.) macht 
sich im zentralen Alpenraum wiederum eine verstärkte Siedlungsaktivität bemerkbar. Zunehmende Eingriffe des Menschen im Waldgrenzbereich seit der Bronzezeit könnten jedoch die Datenlage der Baumbelegkurve verfälschen, was ihre Auswertung als Klimasensor in diesem Zeitabschnitt problematisch macht. Dies erklärt möglicherweise sowohl die mangelhafte Kongruenz der Baumbelegungskurve mit der Sauerstoff-Isotopenkurve (Abb. 2) als auch die nun teilweise gegenläufigen Korrelationswerte mit der Siedlungstätigkeit (Abb. 3), sodass auf eine weitere Auswertung ab 1500 v. Chr. verzichtet wurde.

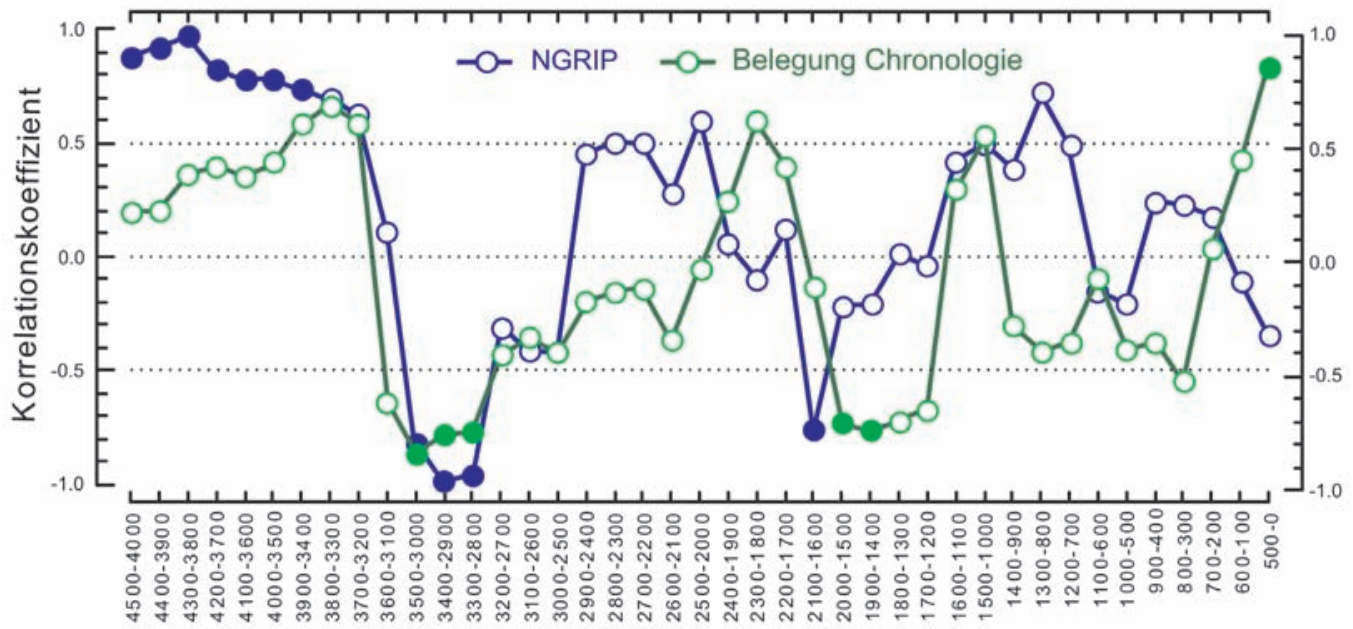

Zeitfenster (Jahre v. Chr.)

Abb. 3: Pearson Korrelation zwischen dem Siedlungsaufkommen im mittleren Alpenraum und (blau) der Sauerstoff-Isotopenkurve von NGRIP (North Greenland Ice Core Project Members 2004) sowie (grün) der Baumbelegungskurve aus mittleren Alpenraum über ein gleitendes Zeitintervall von 500 Jabren. Alle drei Datensätze wurden vor den Korrelationsberechnungen mittels linearer Regression vom linearen Trend bereinigt. Onicht signifikeant, - $95 \%$ signifikant.

Fig. 3: Pearson correlation between settlement activity and (blue) NGRIP $\delta^{18} \mathrm{O}$-values (North Greenland Ice Core Project Members 2004) and (green) the replication record of dated subfossil logs from the central part of the Alps in a moving time window of 500 years. All three data sets are adjusted from the linear trend by linear regression. $\circ=$ not significant, $\bullet=95 \%$ significant. 


\section{Schlussfolgerungen und Hypothesen}

Auffallend ist der Beginn der intensiven Besiedelung der zentralen Alpentäler im 5. Jahrtausend v. Chr., der sowohl mit dem Ende des mittelholozänen Klimaoptimums als auch mit einer ersten Nutzung der ostalpinen Erzlagerstätten (Höppner et al. 2005, Martinek 1997) zusammenfällt. Der Auslöser für diese Migration ist unbekannt. Möglicherweise wird die südliche Erschließung der Alpen durch Bevölkerungsdruck in Kombination mit Extremereignissen (Dürre, Überschwemmungen) ausgelöst. Dafür sprechen Veränderungen im Siedlungsverhalten in der Poebene, wo im 5. Jahrtausend v. Chr. die bevorzugten Siedlungsflächen an niederen Flussterrassen gegenüber höher gelegenen Plätzen auf Moränenhügeln und in Alpentälern aufgelassen werden (Biagi und Nisbeth 1987, Biagi et al. 1993). Dagegen scheidet Bevölkerungsdruck als treibender Faktor für die frühe Aufsiedlung der Alpen von Norden aus, wie jüngste Untersuchungen von Shennan und Edinborough (2007) zeigen.

Betrachtet man das Siedlungsverhalten im zentralen Alpenraum und die Klimaentwicklung von 4500 v. Chr. bis Christi Geburt, so besteht über den Gesamtzeitraum zwischen beiden kein signifikanter Zusammenhang (Abb. 3). Dagegen zeichnet sich für kürzere Zeitabschnitte (500 Jahre) bis in die mittlere Bronzezeit (1500 v. Chr.) annähernd eine Wechselbeziehung ab (Abb. 3). So lässt sich für die Zeit zwischen ca. 4500 bis 3000 v. Chr. ein positiver, von ca. 3000 bis 2800 v. Chr. ein negativer und für ca. 2800 bis 2400 v. Chr. wiederum ein tendenziell positiver Zusammenhang zwischen Klima und Siedlungsverhalten ablesen. Dieses Muster könnte damit erklären werden, dass in den Tallagen nur Klimarückschläge stärkerer Intensität, wie zum Beispiel zwischen 3700 - 3200 v. Chr. (Rotmoos II Schwankung), zu einem Rückgang oder gar zu einer Unterbrechung der Besiedelung führten. Dagegen verursachten kurzfristige und/oder kleinere Schwankungen im Neolithikum zwar Änderungen in der bäuerlichen Wirtschaftsweise (Berglund 2002), jedoch blieb eine signifikante Wirkung auf das Siedlungsverhalten aus.

Dieses resistente Verhalten scheint sich in der frühen bis mittleren Bronzezeit noch weiter zu verstärken, wie die negative Beziehung zwischen Klima- und Siedlungsaufkommen zwischen ca. 2100 bis 1500 v. Chr. (Abb. 3) vermuten lässt. Dies zeigt, dass die Beziehung Siedlungsgeschichte-Klima komplex ist, und viele Faktoren, wie zum Beispiel sozio-ökonomische Einflüsse und der nun intensive Kupferbergbau in diesem Raum (Bartelheim 2007), zu berücksichtigen sind. Hinzu kommt, dass Veränderungen im Niederschlagsverhalten weit schwieriger detektierbar sind als Temperaturschwankungen. Der Niederschlag stellt jedoch 


\section{Klimawandel in Österreich}

eine bestimmende Einflussgröße für die Siedlungstätigkeit dar, denken wir nur an prähistorische Trocken- und Dürreperioden (deMendocal 2001, Hodell et al. 2001, Jalut et al. 2008) oder Überschwemmungen ufernaher Siedlungsräume um ca. 3600 v. Chr. (siehe oben) während der neolithischen Pfahlbaukultur.

\section{Dank}

Die vorliegende Studie wurde durch mehrere Forschungsförderungen an K. Oeggl (FWF P8227-HIS, FWF P16714-G02) und K. Nicolussi (P13065-GEO, EXPICE P15828) ermöglicht.

\section{Literatur}

Arbogast R.-M., Jacomet S., Magny M., Schibler J. 2006: The significance of climate fluctuations for lake level changes and shifts in subsistence economy during the late Neolithic (4300 - 2400 B.C.) in central Europe. Veget Hist Archaeobot 15: 403 - 418

Bartelheim M. 2007:Die Rolle der Metallurgie in vorgeschichtlichen Gesellschaften. Sozioökonomische und kulturhistorische Aspekte der Ressourcennutzung. Ein Vergleich zwischen Andalusien, Zypern und dem Nordalpenraum. Forschungen zur Archäometrie und Altertumswissenschaft 2, Rahden (Westf.), Verlag Marie Leidorf, Rhaden: 471 pp.

Behre K.-E. 1981: The interpretation of anthropogenic indicators in pollen diagrams. Pollen et Spores 23: $225-245$.

Berglund B. E. 2002: Human impact and climate change - synchronous events and a causal link? Quaternary Inernational 105: 7 - 12.

Biagi P., Nisbeth R. 1987: Ursprung der Landwirtschaft in Norditalien. Zeitschrift für Archäologie 21: 11 - 24.

Biagi R., Cremaschi M, Nisbet R. 1993: Soil exploitation and early agriculture in northern Italy. The Holocene 3: 164 - 168.

Burga C., Perret 1998: Vegetation und Klima der Schweiz seit dem jüngeren Eiszeitalter. Thun.

Bortenschlager S. 1970: Waldgrenz- und Klimaschwankungen im pollenanalytischen Bild des Gurgler Rotmooses. Mitteilungen der Ostalpin-dinarischen Gesellschaft für Vegetationskunde 11: 19 - 26.

Bortenschlager I., Bortenschlager S. 1984: Pollenanalytischer Nachweis früher menschlicher Tätigkeit in Tirol. Veröffentlichungen des Museum Ferdinandeum 61: 5 - 12.

deMendocal P.B. 2001: Cultural Responses to Climate Change During the Late Holocene. Science 292: 667 - 673.

Eidenhammer S. 1999: Palynologische Untersuchungen zur holozänen Vegetationsentwicklung im Raum Fieberbrunn und Brandenberg unter besonderer Berücksichtigung des anthropogenen Einflusses. Diplomarbeit Universität Innsbruck. 
Haug G. H., Günther D., Peterson L.C., Sigman D.M., Hughen K.A., Aeschlimann B. 2003: Climate and the Collapse of Maya Civilization. Science 299: 1731 - 1735.

Heiss A.G., Kolfer W., Oeggl K. 2005: The Ulten Valley in South Tyrol, Italy: Vegetation and Settlement History of the Area, and macrofossil Record from the Iron Age Cult Site of St. Walburg. PalynoBulletin 1 (1-2): 63 - 73.

Hodell D.A., Brenner M., Curtis J. H., Guilderson T. 2001: Solar Forcing of Drought Frequency in the Maya Lowlands. Science 292: 1367 - 1370.

Höppner B., Bartelheim M., Huijsmans M., Krauss R., Martinek K.-P., Pernicka E., Schwab R. 2005: Prehistoric copper produktion in the Inn valley (Austria), and the earliest copper in Central Europe. Archaeometry 47/2, 293 - 315.

Hormes A., Müller B. U., Schlüchter C. 2001: The Alps with little ice: evidence for eight Holocene phases of reduced glacier extent in the Central Swiss Alps. The Holocene 11: 255 - 265

Hubmann G. 1994: Palynologische Untersuchung zweier Sedimentprofile aus dem Oberen Inntal mit besonderem Augenmerk auf die anthropogene Beeinflussung. Diplomarbeit Universität Innsbruck.

Iversen, J. 1941: Land occupation in Denmark's Stone Age. Danmarks geol. unders. II, 66: 68 pp.

Iversen, J. 1972: The development of Denmark's Nature since the Last Glacial. Geology of Denmark III. Geological Survey of Denmark V. Series, 7-C: 126 pp.

Jacobson G.L., Bradshaw R.H.W. 1981: The selection of sites for palaeovegetational studies. Quaternary Research 16: 80 - 96.

Jalut G., Dedoubat J.J., Fontugne M., Otto T. 2008: Holocene circum-Mediterranian vegetation changes: Climate forcing and human impact. Quaternary International: DOI:10.1016/ j.quaint.2008.03.012.

Kalis A.J., Merkt J., Wunderlich J. 2003: Environmental changes during the Holocene climatic optimum in central Europe - human impact and natural causes. Quaternary Science Reviews 22: $33-79$.

Kompatscher K.,Oeggl K. 2005: Die spätglaziale und holozäne Vegetationsgeschichte des Überetsch. Palyno-Bulletin 1 (3-4): 31 - 38.

Krapf V. 2001: Palynologische Untersuchungen zur Klima- und Vegetationsgeschichte im Kaunerund Langtaufertal. Diplomarb. Univ. Innsbruck

Martinek K.-P. 1997: Der neue Werkstoff - Metall. In: Archäologisches Landesmuseum (ed): Goldene Jahrhunderte: Die Bronzezeit in Südwestdeutschland. ALManach 2: 16 - 25

Magny M., Leuzinger U., Bortenschlager S., Haas J.N. 2005: Tripartite climate reversal in Central Europe $5600-5300$ years ago. Quaternary Research 65: 3 - 19.

Nicolussi K., Kaufmann M., Patzelt G., Pflicht J. van der, Thurner A. 2005: Holocene tree-line variability in the Kauner-valley, Central Eastern Alps, indicated by dendrochronological analysis of living trees and subfossil logs. Vegetation History and Archaeobotany 14: 221 - 234.

North Greenland Ice Core Project Members 2004: High-resolution record of Northern Hemisphere climate extending into the last interglacial period. Nature 431:147 - 151.

Oeggl, K. 1988: Beiträge zur Vegetationsgeschichte Tirols VII: Das Hochmoor Schwemm bei Walchsee. Berichte des naturwissenschaftlich-medizinischen Vereins in Innsbruck 75: 37 - 60.

Oeggl K. 1998: Palynologische Untersuchungen aus dem Bereich des römischen Bohlenweges bei Lermoos, Tirol. In: Walde E. (Hrsg.): Via Claudia. Neue Forschungen. Institut für Klassische Archäologie der Leopold-Franzens-Universität Innsbruck: 147 - 171. 


\section{Klimawandel in Österreich}

Oeggl K. 1999: Das Luchner Moor - Pollenanalytische Untersuchungen zur Siedlungsgeschichte auf der Gnadenwaldterrasse im Raum Fritzens. In: Gedenkschrift für Aufschnaiter. Heimatkundliche Blätter 8/99: 55 - 64.

Oeggl K., Kofler W., Wahlmüller N. 2005: Pollenanalytische Untersuchungen zur Vegetations- und Siedlungsgeschichte im Montafon. In: Rollinger R. \& Rudigier A. (Hrsg.): Montafon. Geschichte, Kultur und Naturlandschaft. Band 1: Die naturräumlichen Grundlagen: 183 -207.

Pott R., Hüppe J., Remy D., Bauerochse A., Katenhusen O. 1995: Paläoökologische Untersuchungen zu holozänen Waldgrenzschwankungen im oberen Fimbatal (Val Fenga, Silvretta, Ostschweiz). Phytocoenologia 25: 363 - 398.

Schibler J., Jacomet S., Hüster-Plogmann H., Brombacher C. 1997: Economic crash in the 37th and 36th centuries cal. BC in Neolithic lake shore sites in Switzerland. Anthropozoologia 25/26: $553-570$.

Shennan S., Edinborough K. 2007: Prehistoric population history: from the Late Glacial to the Late Neolithic in Central and Northern Europe. Journal of Archaeological Science 34: 1339 - 1345

Schmidl A., Kofler W., Oeggl-Wahlmüller N., Oeggl K. 2005: Land use in the Eastern Alps during the Bronze Age - An archaeobotanical case sturdy of a hill-top settlement in the Montafon (Western Austria ). Archaeometry 47: 455 - 470.

Schmidt R., Koinig K., Thompson R., Kamenik C. 2002: A multi proxy core study of the last 7000 years of climate and alpine land-use impacts on an Austrian mountain lake (Unterer Landschitzsee, Niedere Tauern). Palaeogeography, Plaeoclimatology, Palaeoecology, 187: 101 - 120.

Schmidt, Kamenik C., Schmidt R. 2007. Siliceous algae-based seasonal temperature inference and indicator pollen tracking ca. 4000 years of climate/land use dependency in the southern Austrian Alps. J. Paleolimnol. 38: 541 - 554.

Staffler H., Feichter A. 1999: Subfossile Holzrestfunde in Langtaufers, Vinschgau, Ötztaler Alpen. Der Schlern 73: 161 - 166.

Tinner W., Lotter A. F., Ammann B., Conedera M., Hubschmid P., Leeuwen van J. F. N., Wehrli M. 2003: Climatic change and contemporaneous land-use phases north and south of the Alps 2300 BC to 800 AD. Quarternary Science Reviews 22: 1447 - 1460

Turney C.S.M., Brown H. 2007: Catastrophic early Holocene sea level rise, human migration and the Neolithic transition in Europe. Quaternary Science Reviews 26: 2036 - 2041.

Wahlmüller, N. 1985: Beiträge zur Vegetationsgeschichte Tirols V: Nordtiroler Kalkalpen. Berichte des naturwissenschaftlich-medizinischen Vereins in Innsbruck, 72: 101 - 144.

Wahlmüller N. 1990: Spät- und postglaziale Vegetationsgeschichte des Tschögglberges (Südtirol). Berichte des naturwissenschaftlich-medizinischen Vereins in Innsbruck 77: 7 - 16.

Wahlmüller N. 1993: Palynologische Forschung in den Ostalpen und ihren vorgelagerten Gebieten. Berichte des naturwissenschaftlich-medizinischen Vereins in Innsbruck 80, 81 - 95.

Wahlmüller N. 2002: Die Komperdellalm im Wandel der Jahrtausende. In: Klien R. (Hrsg.): Serfaus. Innsbruck: 71 - 84 .

Walde C. 1999: Palynologische Untersuchungen zur Vegetations- und Siedlungsentwicklung im Raum Kramsach - Brixlegg. Berichte des naturwissenschaftlich-medizinischen Vereins in Innsbruck 86: $61-79$.

Walde C. 2006: Die Vegetations- und Siedlungsgeschichte im Oberen Gericht. Pollenanalytische Untersuchungen des Plemun-Weihers (Fließ, Tirol). In: Walde E., Grabherr G. (Hrsg.): Via Claudia Augusta und Römerstraßenforschung im östlichen Alpenraum. Ikarus 1: 394 - 407. 


\title{
Der Einfluss des Klimas auf die Hochlagennutzung in den südlichen Niederen Tauern (Lungau) während der letzten 4000 Jahre
}

\author{
Schmidt R. ${ }^{1)}$, Kamenik C. ${ }^{2)}$, Roth M. ${ }^{1)}$ \\ 1) Institut für Limnologie, Österreichische Akademie der Wissenschaften, Mondsee, \\ Österreich \\ 2) Oeschger Center for Climate Research \& Institut für Geographie, Universität Bern, \\ Schweiz
}

\section{Abstract}

The impact of climate on alpine land use during the past 4000 years in the southern Niedere Tauern (Lungau)

\begin{abstract}
Alpine pasturing has a long tradition in the Niedere Tauern (Austria). Probably, it started with the use of natural meadows at and above the treeline during Neolithic and Early Bronze times, possibly related to ancient routes crossing the Tauern and/or mining activities. The history of "Almwirtschaft", stored in the sediment archive of Oberer Landschitzsee (ObLAN, $2.067 \mathrm{~m}$ a.s.l., Lungau), suggested a strong relationship with climate. Four pulses of land use, inferred from pollen, occurred during (1) the Early to Middle Bronze, (2) the transition from Late Bronze to Early Iron Age (Hallstatt), (3) Late Iron Age (Celtic) to Roman times, and (4) during high to late medieval times culminating between 1000 and 1400 AD. These four "waves" of land use were separated from each other by cold and wet (snow-rich) climate oscillations. Warm periods with temperatures close to present conditions favored high altitude land use during Roman and high medieval times. Climate seemed to be an important, though complex, trigger of Alpine land use.
\end{abstract}

\section{Einleitung}

Die Almweide in den Alpen blickt auf eine mehrere tausend Jahre lange Tradition und Entwicklung zurück. Pollenanalytische Daten und der Fund des Tiroler Eismannes belegen, dass die Hochlagen der Alpen schon um ca. 5.000 vor heute (Jungsteinzeit) begangen oder als Weide genutzt wurden (Oeggl 1994, Bortenschlager \& Oeggl 2000). Auch in den Niederen Tauern lässt sich die Gegenwart des Menschen in den Hochlagen zumindest bis in diese Zeit zurückverfolgen (Mandl 2003). Auf 
die Nutzung natürlicher Wiesen über der Waldgrenze als Weideland könnten die Menschen dieses Gebietes bei der Benützung alter Saumwege über die Tauern (der Name bedeutet Alpenübergang) gestoßen sein (Mandl-Neumann \& Mandl 2003, Drescher-Schneider 2003), oder aber auch bei der Suche nach Erzen (Bronzezeit), an denen der Lungau reich ist (u.a. Mutschlechner 1967). Aus dieser ursprünglichsten Form der Almweide, wie sie sicher im Neolithikum, wahrscheinlich aber auch noch in der älteren Bronzezeit betrieben wurde, dürfte sich infolge gezielt die Almwirtschaft entwickelt haben. Almwirtschaft, also die zielgerichtete Nutzung auf wirtschaftlicher Basis, ist vom Klima abhängig. Voraussetzungen für eine erfolgreiche Bewirtschaftung sind ein frühes Ende der Schneebedeckung sowie eine möglichst lange bis in den Herbst anhaltende warme und stabile Wetterperiode.

Ablagerungen alpiner Seen stellen wichtige Archive dar, die uns erlauben, die Hochlagennutzung einzelner Gebiete bis über die Zeit historischer Quellen und instrumenteller Klimadaten hinaus zu verfolgen und die Wechselbeziehung Klima/Mensch zu studieren. Multidisziplinäre paläolimnologische Untersuchungsmethoden, die Werkzeuge für die saisonale Klimarekonstruktion beinhalten, helfen uns dabei. Dies soll am Beispiel der Landschitzseen, im Besonderen des Oberen Landschitzsees, dargestellt werden, die intensiv untersucht wurden (Schmidt et al. 2002, 2007, 2008, Kamenik et al. 2005). Die folgende Darstellung fasst die Ergebnisse in verkürzter Form zusammen.

Der Obere Landschitzsee liegt in $2067 \mathrm{~m}$ Seehöhe auf der Südabdachung der Niederen Tauern im hinteren Lessachtal (Lungau) (siehe Schmidt et al., in diesem Band). Aus dem See wurde ein Sedimentkern (ObLAN) gezogen, der mit Hilfe der Radiokarbonmethode (AMS ${ }^{14} \mathrm{C}$ ) datiert wurde. Die Altersangaben sind kalibrierte Alter vor heute. Aufgrund von verbleibenden Datierungsunschärfen sind diese Zeitangaben jedoch Annäherungsdaten. Der menschliche Einfluss wurde mittels Pollen anthropogener Zeigerpflanzen wie z.B. Ampfer, Wegerich, Brennessel, Disteln, sowie Gräsern rekonstruiert. Zudem wurden silikatische Mikrofossilien, Kieselalgen (Diatomeen) und Dauerstadien (Zysten) von Goldalgen (Chrysophyceen), für die Rekonstruktion von Klima- und Umweltvariablen verwendet, welche zuvor kalibriert wurden. Für die Kalibrierung wurden Temperaturmessgeräte (Thermistoren) in 45 Seen der Niederen Tauern aus verschiedenen Höhenlagen und damit Temperaturbereichen exponiert. Entlang dieses Höhen- bzw. Klimagradienten wurden die prozentuellen Anteile dieser Algenreste in Oberflächensedimenten und Sedimentfallen der jeweiligen Seen bestimmt. Die Zeitpunkte der Herbst- (Kieselalgen) und Frühlingsdurchmischung (Goldalgen-Zysten) erklärten statistisch signifikant die 
Verteilung dieser Algengruppen in den 45 Seen (Schmidt et al. 2004, Kamenik \& Schmidt 2005). Mit der in der modernen Paläolimnologie gängigen Methode gewichteter Mittel (,weighted averaging“, WA, siehe u.a. Zusammenfassung bei Birks 1998) wurden für diese Variablen mathematische Modelle, sogenannte Transferfunktionen erstellt (Schmidt et al. 2004, Kamenik \& Schmidt 2005). Mit Hilfe dieser Transferfunktionen wurden aus den fossilen Kieselalgen und Goldalgen-Zysten des Sedimentkerns Oberer Landschitzsee (ObLAN) die erwähnten klimaabhängigen Variablen für die letzten 4000 Jahre rekonstruiert. Weiters wurden aus diesen abgeleiteten Variablen (Frühlings- und Herbstdurchmischung) sowohl die Länge der Eisbedeckung errechnet, als auch die Luftemperatur-Anomalien (Abweichungen gegenüber dem heutigen Messwert) geschätzt. Dabei bedient man sich der Zusammenhänge zwischen Luft- und Wassertemperatur sowie zwischen Lufttemperatur und Meereshöhe (zur genaueren Methode siehe Schmidt et al. 2007). Neben Indikatoren für die Rekonstruktion von Sommerklimaten (wie z.B. Gletscher, Baumringe, Mückenlarven in Seesedimenten) stehen uns damit auch Werkzeuge für die quantitative Rekonstruktion von Frühling-, Herbst bzw. Winterklimaten (Kamenik \& Schmidt 2005) zur Verfügung. Dies ist von Bedeutung, da die sogenannte Nordatlantische Oszillation (NAO), ausgelöst durch Druckunterschiede zwischen Island und den Azoren, im wesentlichen im Winter Einfluss auf die Alpen ausübt (u.a. Wanner et al. 2001, Beniston \& Jungo 2002) und auch in dieser Zeit das Winterregenklima des Mittelmeeres die südlichen Alpenteile beeinflusst (Böhm 2006). Je mehr verschiedene Indikatoren für die Rekonstruktion von Klima- und Umweltveränderungen herangezogen werden, desto treffsicherer wird die Aussage sein. Ein solcher multidisziplinärer ("multi-proxy") Ansatz wurde auch für die Rekonstruktion der Wechselbeziehungen Klima und Landnutzung am Oberen Landschitzsee verwendet (Schmidt et al. 2007, 2008).

\section{Hochlagennutzung und Klima}

Die Pollenanalysen aus dem Oberen Landschitzsee zeigen seit dem Neolithikum Spuren menschlicher Gegenwart, die sich in der Bronzezeit verdichten. Es wurden vier Wellen von Landnutzung festgestellt: (1) Ältere bis Mittlere Bronzezeit (ca. 18001300 vor Christus = BC), (2) Späte Bronze- und Hallstatt-Zeit (ca. 1000 bis 500 BC), (3) Kelten- bis Römerzeit (ca. 300 BC bis 400 nach Christus = AD), (4) Mittelalter (mit Kulmination im Hochmittelalter) (Abb. 1). Mit Ausnahme des Spätmittelalters korrespondieren diese vier Wellen im Wesentlichen mit wärmeren Perioden. 


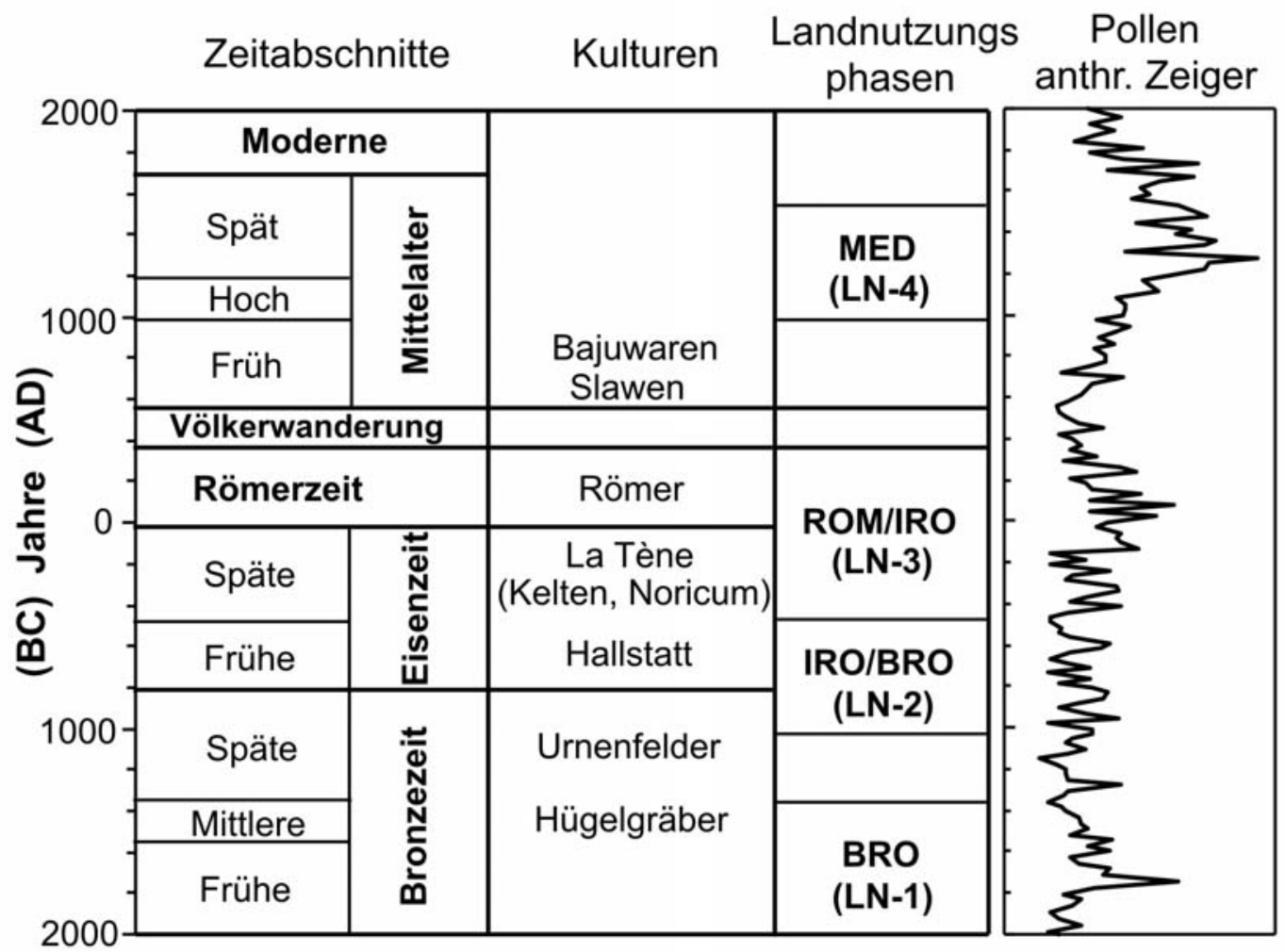

Abb. 1: Übersichtstabelle der Landnutzungsphasen (LN) 1 - 4 im Sedimentkern Oberer Landscbitzsee mit Epochen und Kulturen (BRO = Bronzezeit, IRO = Eisenzeit, ROM = Römerzeit, MED = Mittelalter), sowie Pollen von anthropogenen Zeigerarten in Prozent (nach Schmidt et al. 2007, verändert).

Fig. 1: Land-use (LN) phases $1-4$ indicated in the sediment core Oberer Landschitzsee, with epochs and cultures $(\mathrm{BRO}=$ Bronze, $\mathrm{IRO}=$ Iron age, $\mathrm{ROM}=$ Roman period, $\mathrm{MED}=$ Medieval $)$, and pollen from plants showing human activities.

Die Hochlagennutzung rund um die Landschitzseen scheint während der Bronzezeit zeitweilig intensiver gewesen zu sein als während der folgenden Hallstatt-Zeit (siehe auch Mandl, dieser Band). Mögliche Gründe könnten die Verschiebung des Schwerpunktes zu Handel (Salz, Eisen) während der Hallstatt-Zeit und klimatische Veränderungen gewesen sein. Die Hallstatt-Zeit scheint allgemein feuchter gewesen zu sein, wie Anzeichen erosiver Prozesse in ObLAN erkennen lassen. Beide Abschnitte sind durch eine markante Klimaverschlechterung getrennt, die um ca. 1.500 vor Christus (= BC) einsetzte und um ca. 1.100 BC kulminierte. Diese markante Klimaverschlechterung ist auch in Gletschervorstößen in den Hohen Tauern dokumentiert. 
In der Venediger Gruppe dürfte während der sogenannten Löbben Schwankung (Patzelt \& Bortenschlager 1973) sogar das Ausmaß des neuzeitlichen Gletscherhochstandes von 1850 überschritten worden sein (Nicolussi \& Patzelt 2000). Die Rekonstruktion einer langen Eisdauer im Oberen Landschitzsee (Schmidt et al. 2007).

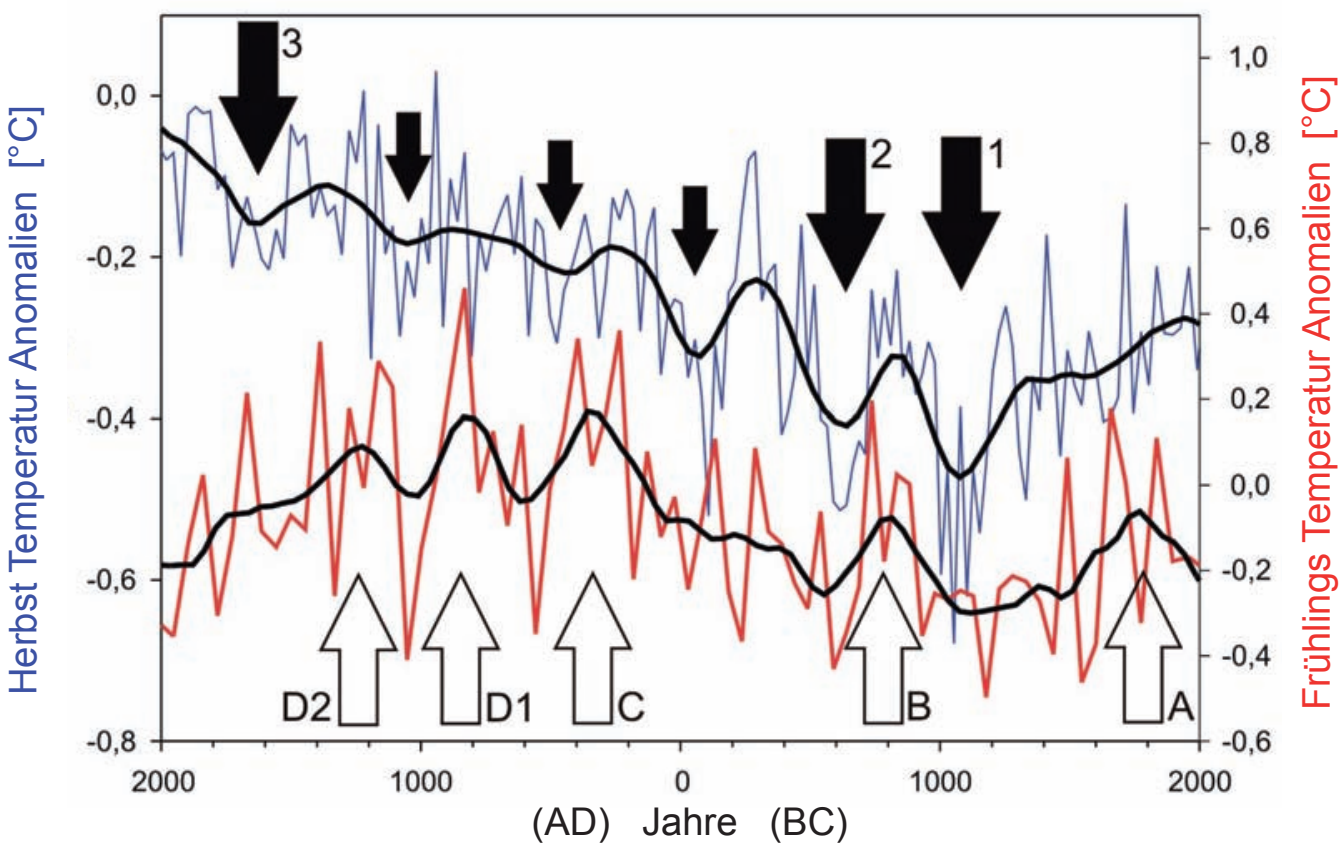

Abb. 2: Oberer Landschitzsee $(2067 \mathrm{~m})$. Aus kalibrierten Kieselalgen und Goldalgen-Zysten abgeleitete mittlere saisonale (Frübling, Herbst) Lufttemperatur-Anomalien der vergangenen 4.000 Jahre (BC $=$ vor Christus, $A D$ $=$ nach Christus) in einem Sedimentkern des Obereren Landschitzsees (Niedere Tauern, Lungau). Die schwarzen Pfeile kennzeichnen die wichtigsten Kälteschwankungen: $1=$ zwischen ca. 1.500 und 1.100 BC, $2=$ um ca. 700 BC, 3 = Little Ice Age (LIA). Zusätzlich sind durch kleinere schwarze Pfeile markiert: Eine Schwankung kurz. vor der Zeitenwende sowie um ca. 1.100 AD, die die Warmzeiten des Mittelalters (D) trennt. Die weissen Pfeile kennzeichnen die wichtigsten Warmzeiten: $A=$ Frühe/Mittlere Bronzezeit, $B=$ Späte Bronzezeit/Hallstatt, $C=$ Römerzeit, $D=$ Mittelalter $(1=$ Frühes, $2=$ Hochmittelalter). Beachtenswert ist die zumeist enge Koppelung der beiden Temperaturkurven in den beiden Jahrtausenden vor Christus und die zunehmende Entkoppelung beginnend im Mittelalter und kulminierend wäbrend LIA (nach Schmidt et al. 2007, verändert).

Fig. 2: Oberer Landschitzsee $(2067 \mathrm{~m})$. Mean seasonal (spring, autumn) air temperature anomalies of the past 4000 years $(\mathrm{BC}=$ before Christ, $\mathrm{AD}=$ after Christ), inferred from calibrated diatoms and chrysophyte cysts in a sediment core from Oberer Landschitzsee (Niedere Tauern, Lungau). The black arrows $1-3$ indicate the major climate deteriorations. Smaller black arrows indicate a fluctuation just before 0 , and another one at ca. $1100 \mathrm{AD}$, separating the medieval warm periods (D). The white arrows indicate the major warm periods: $\mathrm{A}=$ Early/Middle Bronze, $\mathrm{B}=$ Late Bronze/ Hallstatt, $\mathrm{C}=$ Roman time, $\mathrm{D}=$ medieval times $(1=$ Early and $2=$ High Medieval $)$. Note the coupling of both inferred temperature curves during the two millennia BC and their decoupling, starting during medieval times, and culminating during LIA. 
steht damit im Einklang. Eine kühlere und feuchtere Phase zwischen ca. 750 und 350 BC trennt wiederum die Landnutzungsphase der Hallstatt von jener der Kelten bis zur Römerzeit. Höhere Seespiegelstände in Seen der nordwestlichen Alpen (u.a. Magny 2004) und Einbrüche in den Sauerstoff-Isotopen-Kurven $\left(\delta^{18} \mathrm{O}\right)$ Grönländischer Eiskerne (z.B. GRIP, Dansgaard et al. 1993; Grootes et al. 1993; Vergleich verschiedener Eiskerne: Vinther et al. 2006) zeigen die überregionale Dimension dieser beiden für ObLAN nachgewiesenen Kaltphasen. Die Keltenzeit könnte, nach der Temperaturableitung im oberen Landschitzsee zu schließen, durch stärkere saisonale Unterschiede zwischen wärmeren Herbsten und kühleren (schneereichen?) Frühlingen (siehe Little Ice Age, LIA), sowie eine allgemeine Abkühlung vor der Zeitenwende charakterisiert sein, was auch die niedrige Baumgrenze in dieser Zeit (Nicolussi et al. 2005) erklären würde.

Eine Intensivierung (siehe Anteil anthropogener Pollenzeiger in Abb. 1), aufbauend auf der Tradition der Kelten, erfuhr die Almwirtschaft während der Römerzeit. Dafür dürften zwei Voraussetzungen maßgeblich gewesen sein: (1) die Römerzeit zeigt in der Klimarekonstruktion von ObLAN ähnliche Temperaturen wie heute, was die Hochlagenbeweidung begünstigte; (2) entlang der Mur führte eine wichtige NordSüd-Verbindung der Römer. Straßensiedlungen im Lungau (z.B. Immurium, das heutige Moosham, Fleischer \& Moucka-Weitzel 1998) dürften wahrscheinlich auch den Güteraustausch mit den umliegenden Almen gefördert haben. Diese Landnutzungsperiode endet mit dem Zusammenbruch des Römischen Reiches zwischen 476 und 488 AD und der Völkerwanderung. Sowohl klimatisch (feuchterer Abschnitt) als auch siedlungsgeschichtlich handelt es sich um eine Periode der Instabilität.

Im Mittelalter beginnt die jüngste Welle der Weidewirtschaft an den Landschitzseen, die zwischen ca. 1.000 bis 1.400 AD (Hochmittelalter) gipfelte. Änderungen in der Zusammensetzung des Pollenspektrums von ObLAN lassen auf Rinderweiden schließen. Im Vergleich mit der römerzeitlichen Almweide (Schafe, Ziegen?) sind Nachweise von Pflanzenarten stickstoffliebender (nitrophiler) Lägergesellschaften, von Hochstauden, sowie Heide- (Besenheide), Wiesenpflanzen und Farne (Adlerfarn) häufig. Mit Ausnahme des Spätmittelalters während der sogenannten Kleinen Eiszeit handelte es sich um eine Reihe warmer Perioden, vor allem im Zeitraum 1.000 bis $1.400 \mathrm{AD}$, die auch als Mittelalterliche Wärmeperiode (MWP) zusammengefasst werden. Die Klimarekonstruktion von ObLAN zeigt jedoch schon zwischen ca. 700 und $1.000 \mathrm{AD}$ ein ähnliches, mit heutigen Temperaturen vergleichbares, Niveau, das durch kurzfristige kühle und feuchtere Phasen unterbrochen wurde (Nicolussi \& Patzelt 2000, Nicolussi et al. 2005). Eine solche führte um ca. 1.100 AD 
am Oberen Landschitzsee zu verstärktem Bodenabtrag (Erosion). Im Gegensatz zu den älteren Siedlungsabschnitten entschied seit dem Hochmittelalter jedoch nicht mehr alleine das Klima über die Nutzung der Hochlagen. Die Nutzungsstrukturen wurden durch andere Faktoren überlagert, wie möglicherweise Änderungen in den Besitzverhältnissen. So wurde der Lungau im 13. Jahrhundert Teil des Erzbistums Salzburg (u.a. Keusch 1948). Einflüsse, die sich aus Veränderungen in den Besitzverhältnissen ergaben, konnten auch in anderen Alpenseen nachgewiesen werden (Kamenik et al. 2000).

Neben der Variabilität saisonaler Klimate geben die paläolimnologischen Befunde Hinweise auf langfristige Klimaveränderungen. Während der zwei Jahrtausende vor Christus waren die aus zwei verschiedenen Indikatororganismen abgeleiteten Frühlings- und Herbsttemperaturen von ObLAN überwiegend signifikant korreliert, sodass man auf eine vergleichbare saisonale Niederschlagsaktivität schließen könnte. Eine erhöhte Tiefdrucktätigkeit im nördlichen Mittelmeer, vor allem aber die so genannte Genua-Zyklone, beeinflussen heute den Niederschlag in den südlichen Alpen, vor allem in der Zeit von Herbst bis Frühling (Böhm 2006). Diese Tiefdrucktätigkeit steht wiederum in Zusammenhang mit Tiefdrucksystemen des Nordatlantiks und damit auch mit der Nordatlantischen Oszillation (NAO), die das heutige Winterklima in den Alpen beeinflusst (u. a. Wanner et al. 2001, Beniston \& Jungo 2002). Als Arbeitshypothese wurde postuliert (Schmidt et al. 2004), dass sich zwischen 5.000 und $4.000 \mathrm{v}$. h. der Einfluss des mediterranen Winterregenklimas in den südlichen Alpen verstärkte und dieser Einfluss auch noch in den nächsten beiden Jahrtausenden anhielt (Schmidt et al. 2007, 2008, sowie in diesem Band). Während des Mittelalters setzte eine Entkoppelung der abgeleiteten mittleren Frühlings- und Herbsttemperaturen ein, die möglicherweise damit zusammenhängt, dass sich der Hauptniederschlag auf den Frühling verlagerte. Dieser Trend setzte sich scherenförmig verstärkend in die folgende sogenannte Kleine Eiszeit (Little IceAge, LIA) fort, die sich dadurch von den beiden älteren größeren Kälteschwankungen (1 und 2, siehe Abb. 2) vor Christus unterscheidet. Dies könnte auf großräumige Veränderungen in den Zirkulationsströmungen hindeuten, die bewirkten, dass der Lungau im Vergleich zu den beiden Jahrtausenden vor Christus wieder (siehe Beitrag über das ältere Holozän in diesem Band) kontinentaler wurde. Gestützt wird diese Hypothese u.a. durch Pollenanalysen, die darauf schließen lassen, dass die Buche während des Mittelalters aus dem Lungau verschwand und die Tanne heute nur mehr Reliktstandorte aufweist. Beide sind Charakterbäume der humiden (schneereichen) Außenalpen. Veränderungen in den geochemischen und mineralo- 
gischen Charakteristika von ObLAN lassen ebenfalls auf schneeärmere, kontinentalere Verhältnisse seit dem Mittelalter schließen (Schmidt et al. 2008).

\section{Schlussfolgerungen}

Die quantitative Rekonstruktion saisonaler Klimate mit Hilfe kalibrierter aquatischer Organismen hat wesentlich zum Verständnis der Klimaentwicklung im Holozän beigetragen. In Verbindung mit einem multidisziplinären paläolimnologischen Ansatz konnten die Wechselbeziehungen Klima/Mensch während des jüngeren Holozäns in den Hochlagen der südlichen Niederen Tauern (Lungau) rekonstruiert werden. Die vier Wellen der Hochlagennutzung am Oberen Landschitzsee während der letzten 4.000 Jahre waren, mit Ausnahme der jüngeren Zeit, eng mit Wärmeperioden verknüpft, die durch Kälteschwankungen voneinander getrennt sind. Ausgangspunkt der heutigen Almwirtschaft dürfte die Nutzung natürlicher Wiesen an und über der Waldgrenze als Weideland gewesen sein (Neolithikum, ältere Bronzezeit). Eine Intensivierung der Hochlagennutzung erfolgte, aufbauend auf der Tradition der Kelten, während der Römerzeit und die Kulmination im Hochmittelalter. Beide waren überwiegend Wärmeperioden mit ähnlichen Temperaturen wie heute. Der Langzeittrend deutet ab dem Mittelalter wiederum auf eine Entwicklung zu kontinentaleren Klimaverhältnissen im Lungau hin, wie sie zuletzt das sogenannte „Holozäne Klimaoptimum“ kennzeichneten (siehe Beitrag in diesem Band). Ein Grund für diese langfristige Klimaänderung gegenüber den zwei Jahrtausenden vor Christus könnten veränderte Zirkulationsströmungen sein, wie zum Beispiel die Nordatlantische Oszillation (NAO) und die Genua-Zyklone, die beide das Winterklima in den Alpen beeinflussen. Aus dem Zusammenspiel zwischen kontinentalen Verhältnissen und menschlichem Einfluss (Rodung) entwickelten sich die für die Zentralalpen charakteristischen lärchen- und zirbenreichen Almen.

\section{Dank}

Die Untersuchungen waren Teil der Projekte des Österreichischen Forschungsfonds (FWF, P14912-B06) und des Programms Alpenforschung der Österreichischen Akademie der Wissenschaften (Projekt CLIM-LAND). 
Einfluss des Klimas auf die Hochlagennutzung in den südlichen Niederen Tauern

\section{Zitierte Literatur}

Beniston M., Jungo P. 2002. Shifts in the distribution of pressure temperature and moisture and changes in the typical weather patterns in the Alpine region in response to the behaviour of the North Atlantic Oscillation. Theor. Appl. Clim. 71: 29-42.

Birks H.J.B. 1998. Numerical tools in palaeolimnology - Progress, potentialities, and problems. J. Paleolimnol. 20: 307-332.

Böhm R. 2006. Reconstructing the climate of the 250 years of instrumental records at the northern border of the Mediterranean (The Alps). Il Nuovo Cimento 29: 13-19.

Bortenschlager S., Oeggl K. 2000. The man in the ice. IV The iceman and his natural environment. Springer Humanbiology, Vienna, Austria, pp164.

Dansgaard W., Johnsen S.J., Clausen H.B., Dahl-Jensen D., Gundestrup N.S., Hammer C.U., Hvidberg C.S., Steffensen J.P., Sveinbjörns-Dottir A.E., Jouzel J., Bond G. 1993. Evidence for general instability of past climate from a 250-kyr ice-core record. Nature 364: 218-220.

Drescher-Schneider R. 2003. Pollenanalytische Untersuchungen an einem Bodenprofil in Zusammenhang mit dem urgeschichtlichen Brandopferplatz auf dem Sölkpass (1780 m NN, Niedere Tauern, Steiermark). In: Mandl F. (ed.), Sölkpass. Ein 6000 Jahre alter Saumpfad über die Alpen. ANISA, 89-112.

Fleischer R., Moucka-Weitzel V. 1998. Die römische Strassenstation Immurium-Moosham im Salzburger Lungau. Archäologie in Salzburg, Bd. 4, pp. 320.

Grootes P.M., Stuiver M., White J.W.C., Johnsen S., Jouzel J. 1993. Comparison of oxygen isotope records from the GISP2 and GRIP Greenland ice cores. Nature 366: 552-554.

Kamenik C., Schmidt R. 2005. Chrysophyte resting stages a tool for reconstructing winter/spring climate from Alpine lake sediments. Boreas 34: 477-498.

Kamenik C., Koinig K.A., Schmidt R. 2005. Potential effects of pre-industrial lead pollution on algal assemblages from an Alpine lake. Verh. Internat. Verein. Limnol 29: 535-538.

Kamenik C., Koinig K.A., Schmidt R., Appleby P.G., Dearing J.A., Lami A., Thompson R., Psenner R. 2000. Eight-hundred years of environmental changes in a high alpine lake (Gossenköllesee, Tyrol) inferred from sediment records. J. Limnol. 59: 43-52.

Keusch P. 1948. Geschichte des Lungaus. Salzburger Kulturvereinigung, pp. 18.

Magny M. 2004. Holocene climate variability as reflected by mid-European lake-level fluctuations and its probable impact on pre-historic human settlements. Quat. Int. 113: 65-79.

Mandl F. 2003. Almen im Herzen Österreichs. Dachsteingebirge, Nieder Tauern, Salzkammergut, Totes Gebirge. ANISA, pp. 312.

Mandl-Neumann H., Mandl F. 2003. Der Sölkpass in Geschichte und Gegenwart. In: Mandl F. (ed.), Sölkpass. Ein 6000 Jahre alter Saumpfad über die Alpen. ANISA, 5-43.

Mutschlechner G. 1967. Über den Bergbau im Lungau. Mitt. Ges. für Salburger Landeskunde 107: 129-168.

Nicolussi K., Kaufmann M., Patzelt G., van der Pflicht J., Thurner A. 2005. Holocene treeline variability in the Kauner Valley, central Eastern Alps indicated by dendrochronological analysis of living trees and subfossil logs. Veget. Hist. Archaeobot. 14: 221-234.

Nicolussi K., Patzelt G. 2000. Untersuchungen zur Holozänen Gletscherentwicklung von Pasterze und Gepatschferner (Ostalpen). Z. Gletscherkde. Glazialgeol. 36: 1-87. 


\section{Klimawandel in Österreich}

Oeggl K. 1994. The palynological record of human impact in highland zone ecosystems. In: Biagi P., Nandris J. (eds), Highland exploitation in southern Europe. Monograf. Nat. Bresciana 20: 107-122.

Patzelt G., Bortenschlager S. 1973. Die postglazialen Gletscher- und Klimaschwankungen in der Venedigergruppe (Hohe Tauern, Ostalpen). Z. Geomorph. N.F. Suppl. Bd. 16: 25-72.

Schmidt R., Roth M., Tessadri R., Weckström K. 2008. Disentangling Late-Holocene climate and land use impacts on an Austrian alpine lake using seasonal temperature anomalies, ice-cover, sedimentology, and pollen tracers. J. Paleolimnol 40: 453-469.

Schmidt R., Kamenik C., Roth M. 2007. Siliceous algae-based seasonal temperature inference and indicator pollen tracking ca. 4,000 years of climate/land use dependency in the southern Austrian Alps. J. Paleolimnol. 38: 541-554.

Schmidt R., Kamenik C., Kaiblinger C., Hetzel M. 2004. Tracking Holocene environmental changes in an alpine lake sediment core: application of regional diatom calibration, geochemistry, and pollen. J. Paleolimnol. 32: 177-196.

Schmidt R., Koinig K.A., Thompson R., Kamenik C. 2002. A multi proxy core study of the last 7000 years of climate and alpine land-use impacts on an Austrian mountain lake (Unterer Landschitzsee, Niedere Tauern). Palaeogeo., Palaeoclimat., Palaeoecol. 187: 101-120.

Vinther B.M., Clausen H.B., Johnsen S.J., Rasmussen S.O., Andersen K.K., Buchardt S.L., Dahl-Jensen D., Seierstad I.K., Siggaard-Andersen M.-L., Steffensen J.P., Svensson A., Olsen J., Heinemeier J. 2006. A synchronized dating of three Greenland ice cores throughout the Holocene. J. Geophys. Res. 111, D13102, DOI:10.1029/2005JD006921, 2006.

Wanner H., Brönnimann S., Casty C., Gyalistras D., Luterbacher J., Schmutz C., Stephenson D.B., Xoplaki E. 2001. North Atlantic oscillation concepts and studies. Surv. Geophys. 22: 321-382. 


\title{
Hallstatts bronzezeitliche Almen
}

\author{
Franz Mandl
}

ANISA, Verein für alpine Forschung, Raiffeisenstraße 92, A-8967 Haus i. E.

\section{Abstract}

\begin{abstract}
Alpine pastures of Hallstatt during the Bronze Age
Alpine pasturing at the Dachstein plateau around Hallstatt (Salzkammergut) started during the Bronze Age at about 1700 BC. Salt mining in Hallstatt and pasturing during the Bronze Age were closely related. Pasturing started during the warm period of the early to middle Bronze Age, however intensified between ca. 1400 and 1200 BC, when climate deteriorated. This contradiction could be explained by the need for food supply of the growing population of the salt mining community of Hallstatt, where space for agriculture is limited. When climate deterioration culminated at about $1100 \mathrm{BC}$, alpine pasturing came to an end. It recovered neither during the climate amelioration of the transition Late Bronze (Urnenfelderzeit/ Hallstatt) nor during the Celtic epoche. This could be due to the increasing importance of trade related to salt industries. However, more interdisciplinary work and novel approaches are necessary to fully understand the complex interactions between climate, alpine pasturing, and socio-economy during the prehistoric development of Hallstatt.
\end{abstract}

\section{Einleitung}

Die Ursprünge der Almweide lassen sich in den Alpen bis in das Neolithikum zurückverfolgen. Grundsätzlich wird die These vertreten, dass für prähistorische Weiden („Urweiden“) vor allem die Gräser an der Waldgrenze und darüber genutzt wurden und erst ab der Bronze-, spätestens aber ab der Eisenzeit dank verbesserter Rodungsmöglichkeiten auch tiefer gelegene Waldbereiche gerodet wurden.

Die Nutzungsstrukturen, die von der Almweide zur Almwirtschaft führten, sind vielfältig. Die in historischer Zeit am längsten verwendete Form der Almwirtschaft ist die Mischform aus Fleischzucht und Erzeugung von Milchprodukten. Der Begriff „Almwirtschaft“ deckt unterschiedliche Formen der Weidenutzung ab. Die „traditionelle“ Almwirtschaft verfügt über eine Sennerei, in der die Milch vor Ort verarbeitet wird. Es gibt aber auch Milchalmen, von denen die Milch zur Verarbeitung ins Tal transportiert wird. Die reine Viehhaltung für die Fleischproduktion, die Sömmerung nicht milchgebenden Viehs und die vielen Varianten mehr oder weniger 
mobiler Tierhaltungsformen, bei denen Hirten mit Hunden die Herden begleiten, zählen ebenso dazu. Die Alpengräser und -kräuter stärken das Weidevieh und das Höhenklima härtet es ab. Nicht nur Rinder, Pferde, Schafe und Ziegen, sondern auch Schweine wurden auf den Almen gesömmert, wo sie mit Molke, Abfällen und Alpenampfer gefüttert wurden. Die Monate der Almbewirtschaftung entlasteten die Talgründe um den Heimhof und ermöglichten daher Futtergewinnung für die Winterbevorratung und Ackerbau. Alm und Heimhof bildeten eine untrennbare wirtschaftliche Einheit, ohne die ein Überleben in inneralpinen Regionen in früheren Zeiten der bäuerlichen Selbstversorgung undenkbar gewesen wäre (Mandl 1999, 2006 a, b, 2007). Weitere wichtige Produkte entstanden aus der Verwertung der Wolle, der Felle, der Hörner und der Knochen des Weideviehs.

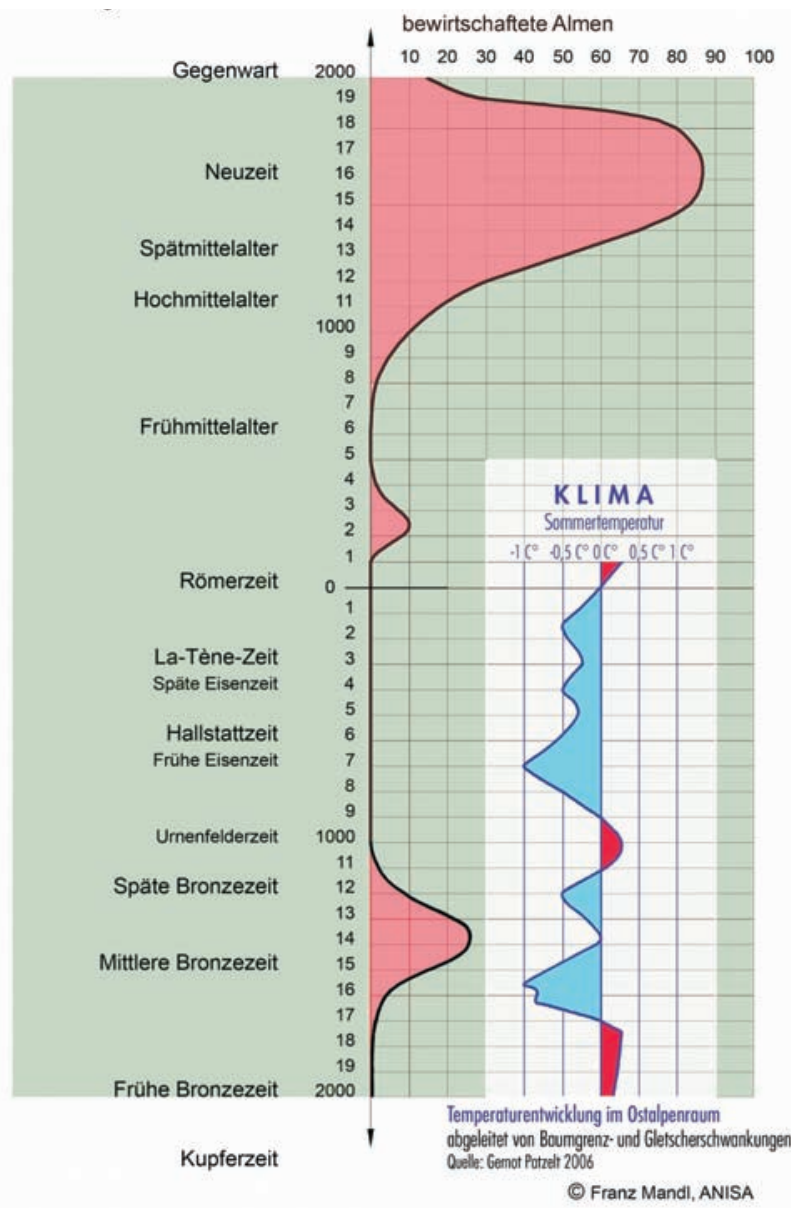

Abb. 1. Zeittafel mit Almwirtschaft am Dachstein und Klimaentwicklung (vereinfacht nach Patzelt 2006)

Fig. 1. Time table of pasturing at Dachstein and rough climate development (after Patzelt, 2006) 
In Hallstatt (Salzkammergut) wurde, wie neue Ausgrabungen belegen, bereits in der mittleren Bronzezeit Salz im Berg abgebaut (Barth \& Reschreiter 2005). Hallstatt verfügte jedoch aufgrund seiner geografischen Lage zwischen dem Dachsteingebirge und dem Hallstätter See über wenige landwirtschaftlich nutzbare Flächen, die Bergleute mussten aber mit Nahrungsmitteln versorgt werden. Das östliche Dachsteinplateau mit einer Fläche von $280 \mathrm{~km}^{2}$ ist ein ideales Almengbiet. Daher konnte dort ein Teil der Nahrungsmittel für Hallstatt produziert werden. Wie hoch dieser Anteil war, ist beim derzeitigen Forschungsstand noch ungewiss.

Neben den natürlichen Wiesen im Waldgrenzbereich und darüber fanden sich „Urweiden“ auch innerhalb des Waldgebietes in Karstmulden, in denen Wasser stauende tonige Sedimente und eingetragene Pflanzenreste die Humusbildung fördern. Diese Karstmulden von unterschiedlichster Größe, wie sie am Dachstein über 1200 $m$ Höhe auftreten, weisen ein eigenes Kleinklima auf. Schnee bleibt im Frühjahr dort länger liegen und Reif bildet sich bereits im Spätsommer, was den Strauch- und Baumbewuchs behindert, sodass natürliches Grünland entsteht. Man ersparte sich dort die mühsame Rodungsarbeit. Die Beweidung förderte zudem die Ausbreitung der Weideflächen. Bronzezeitliche Hüttenreste findet man vorwiegend in den Karstmulden.

\section{Methoden}

Die Hüttenreste sind an ihren heute verschliffenen Steinkränzen zu erkennen. Diese können aber im Steingewirr des Karsts nur von einem geübten Auge ausgemacht werden. Langjährige Feldforschungen, wie sie von der ANISA, Verein für alpine Forschung initiiert und betrieben wurden, waren daher die Voraussetzung für deren Entdeckung. Der Altersnachweis erfolgte durch Radiokohlenstoffdatierungen von Holzkohle, durch einige wenige Bronzegegenstände sowie Keramikfragmente.

\section{Ergebnisse}

Im Rahmen der inzwischen 30 Jahre dauernden Forschungen konnten 23 urgeschichtliche Siedlungsstationen nachgewiesen werden. Mit Hilfe der Radiokohlenstoffdatierung (AMS, Accelerator Mass Spectrometry) ist die Mehrzahl davon der mittleren Bronzezeit zuzuweisen. Weitere 10 Siedlungsorte warten auf eine Beprobung. Sie 
alle befinden sich zwischen $1300 \mathrm{~m}$ und $2100 \mathrm{~m}$ Höhe. Aus den bisher datierten urgeschichtlichen Hüttenresten auf dem Dachsteinplateau lässt sich kein direkter Zusammenhang zwischen Alter und Höhenlage ableiten. Dies könnte damit zusammenhängen, dass sich das Angebot natürlichen Weidelandes nicht alleine auf den Waldgrenzbereich und darüber beschränkte, sondern dass auch die schon erwähnten „Urweiden“ in Karstmulden genutzt wurden. Die räumliche Verteilung der urgeschichtlichen Almen auf der Dachsteinhochfläche ist hingegen auffallend gegliedert, denn die Funddichte nimmt in Richtung Hallstatt zu. Die Datierungen belegen den Beginn der bronzezeitlichen Almwirtschaft um ca. 1700 v. Chr., die
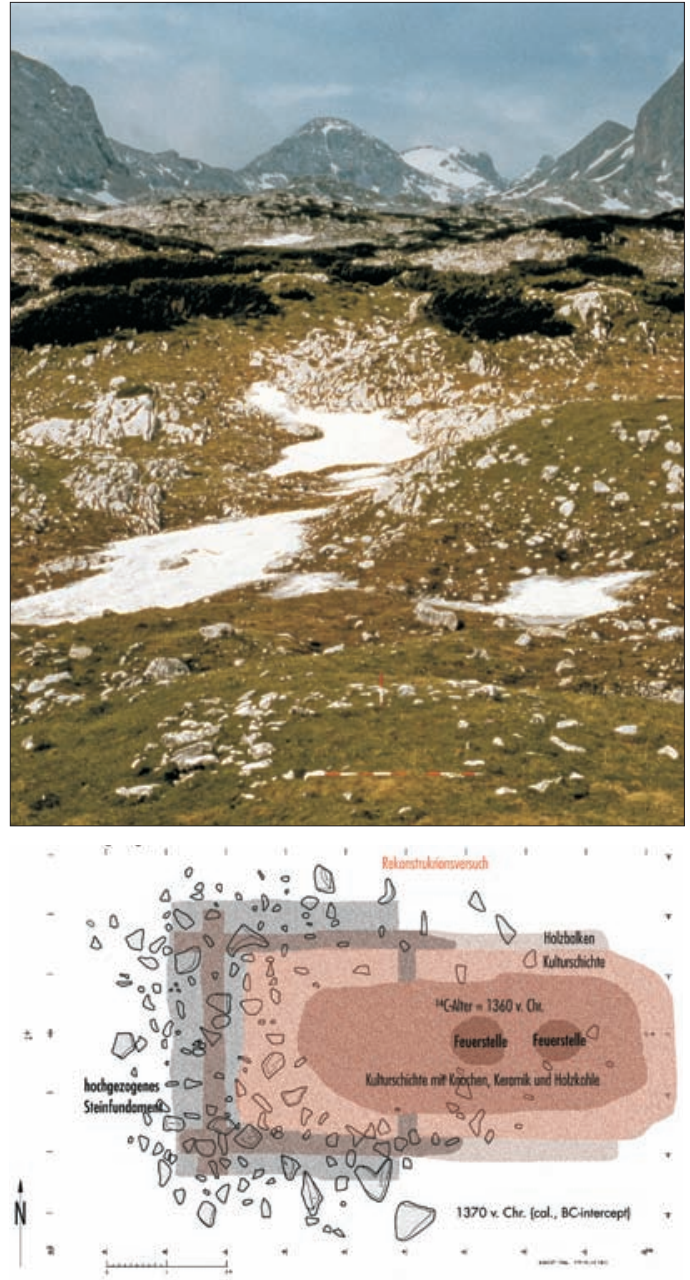

Abb. 2: Lackenofengrube, Hüttenfundament (Dachsteingebirge, $1995 \mathrm{~m}$ ).

Fig. 2: Lackenofengrube, foundation of a former alpine hut (Dachstein Mountains, $1995 \mathrm{~m})$.

Abb. 3: Grundriss der Hütte in der Lackenofengrube (Dachsteingebirge, $1995 \mathrm{~m}$ ).

Fig. 3: Lackenofengrube, ground plan of the former alpine hut (Dachstein Mountains, $1995 \mathrm{~m})$. 
größte Datendichte findet sich zwischen 1440 und 1260 v. Chr. Das ist auch der Höhepunkt des bronzezeitlichen Salzbergbaues im Hallstätter Christian-Tusch-Werk, in dem die inzwischen berühmt gewordene Holzstiege (1344 v. Chr.) und weitere bis 3460 Jahre alte Hölzer gefunden und mit Hilfe der Dendrochronologie (Salz-Reich. Kern A. 2008) datiert wurden. Der Salzbergbau der mittleren Bronzezeit korreliert mit der Almbewirtschaftung auf dem Dachsteingebirge. Zur Frage des Hallstätter Siedlungswesens hat Thomas Stöller (Stöller 1996.) bronzezeitliche Kulturschichten, die auf Siedlungen hinweisen, im Ortsbereich von Hallstatt, im Friedlfeld, in der Lahn und im Echerntal nachgewiesen. Dort könnten sich mögliche Heimgüter für die erwähnten Almen befunden haben.

Bisher wurden nur zwei bronzezeitliche Almhüttenreste ausgegraben, und zwar in der Lackenofengrube $(1995 \mathrm{~m})$ und in der Königreichalm $(1730 \mathrm{~m})$ (Tiefengraber 2007). Nur in der Lackenofenhütte konnten bisher Knochenreste von Rind, Schwein, Hund, Ziege oder Schaf und Pferd geborgen werden. Dabei handelt es sich ausschließlich um Knochen von Jungtieren (Kanelutti/Rabeder 1990), was vermuten lässt, dass die Tierhaltung temporär und nicht ganzjährig erfolgte. Beide Hütten waren wahrscheinlich Blockbauten, die auf Fundamenten aus geschlichteten Steinen (Steinkranz) mit teils bis zu $1 \mathrm{~m}$ hohen Trockenmauern errichtet worden waren. Die Steinmauern waren an der Rückwand, der Wetterseite, mächtiger, während die Hütten nach Süden und Osten offen waren. Vor dem Eingang befanden sich jeweils zwei Feuergruben (Mandl 2007). Es finden sich auch Hinweise auf andere Wirtschaftsformen. Funde von Lappenbeilen aus Bronze im Hochwald des Dachsteingebirges könnten auf eine Holzwirtschaft für den Hüttenbau und sogar den Salzbergbau hinweisen. Die Funde von Lanzenspitzen aus Bronze deuten auf jagdliche Tätigkeiten. Wahrscheinlich ist auch ein Saumhandel mit Salz und Gegenware, denn über das Dachsteingebirge führte der kürzeste Weg nach Süden. Für diese Tätigkeiten benötigten die Menschen Raststationen und Nahrungsmittel, zu deren Versorgung vermutlich auch einige Almen auf dem Dachsteingebirge dienten.

Um 1100 v. Chr. nahm die Intensität der Almwirtschaft offensichtlich stark ab, nach 1000 v. Chr. ist sie nicht mehr nachweisbar. Gleichzeitig ist ein Rückgang des Hallstätter Salzbergbaus festzustellen (Salz-Reich, in Kern et al. 2008). Nach den Datierungen vom Dachstein zu schließen, fällt der Beginn der bronzezeitlichen Almwirtschaft um ca. 1700 v. Chr. in eine Wärmeperiode, wie dendrochronologische (Nicolussi et al. 2005) und paläolimnologische Befunde (Schmidt et al. 2007) in den Ostalpen belegen. Die Anzahl der Funde verdichtet sich aber zwischen ca. 1400 und 1300 v. Chr., in einer Zeit beginnender klimatischer Instabilität. Dieser 
Widerspruch könnte damit erklärt werden, dass die Expansion des bronzezeitlichen Salzbergbaues vermutlich zu einer starken Zunahme der Bevölkerung und damit zu verstärktem Nahrungsbedarf führte. Daher könnte die Almwirtschaft am Dachstein trotz des ungünstigeren Klimas intensiv betrieben worden sein, um die Versorgung von Hallstatt zu sichern. Sowohl auf den Hochalmen als auch in den niedrigeren waldfreien Karstmulden dürfte sich mit zunehmender Klimaverschlechterung der Weidebetrieb auf den Sommer beschränkt haben. Die Ursache für das folgende gänzliche Erliegen der Almwirtschaft am Dachstein könnte eine massive Klimaverschlechterung ab ca. 1100 v. Chr. (Schmidt et al. 2007) sein, während der der Hallstätter Gletscher den Taubenriedel auf dem Dachsteinplateau um 900 v. Chr. erreicht hat (Patzelt 2003), sie könnte aber auch in einer grundlegenden politischen und wirtschaftlichen Veränderung an der Wende der jüngeren Bronzezeit (Urnenfelderzeit) zur Hallstattzeit in Hallstatt selbst zu suchen sein. Die Ergebnisse von Hallstatt zeigen Übereinstimmungen bzw. Assoziationen mit jenen von Schmidt et al. (2007) aus den benachbarten Niederen Tauern: Eine erste Blüte zeichnet sich auch hier um 1700 v. Chr. während einer Wärmezeit ab. Es folgte eine markante Klimaverschlechterung, die um ca. 1100 v. Chr. gipfelte. Gegenüber der mittleren Bronzezeit sind für die eigentliche Hallstattzeit (frühe Eisenzeit) bisher keine Siedlungen nachweisbar. Die grundsätzlich gute Übereinstimmung zwischen Hochlagenbeweidung und Wärmeperioden während der letzten 4000 Jahre in den Niederen Tauern wurde im Spätmittelalter durchbrochen. Während der ungünstigen Klimabedingungen der sogenannten „Kleinen Eiszeit“ wurden aus vielleicht ebenfalls sozio-ökonomischen Gründen alpine Nutzungsstrukturen aufrechterhalten. Die Almwirtschaft wurde, soweit wir dies aus der heutigen Sicht der Forschungsergebnisse beurteilen können, weder in der Hallstattzeit (750-450 v. Chr.) noch in der Kelten/La-Tène-Zeit (450 - 15. v. Chr.) wieder aufgenommen. Eine Erklärungsmöglichkeit wäre, dass dank des Salzhandels während der Hallstattzeit Lebensmittel in größerem Ausmaß importiert werden konnten.

\section{Schlussfolgerungen}

Die Ergebnisse deuten darauf hin, dass sich die Almwirtschaft auf dem Dachsteinplateau aus der Tradition der bronzezeitlichen Hochlagennutzung unter klimatisch günstigen Bedingungen (um ca. 1700 v. Chr.) entwickelt hat und in Folge unter zunehmend instabilen klimatischen Bedingungen sogar bis in die späte Bronzezeit 
intensiviert wurde, um die Versorgung der Bergwerkssiedlung von Hallstatt zu gewährleisten. Erst um etwa 1000 v. Chr. mussten offensichtlich auch die auf den Sommer beschränkten natürlichen Nutzflächen aufgegeben werden. Sehr wahrscheinlich waren die markante Klimaverschlechterung am Ende der Bronzezeit und darauf folgende politische oder wirtschaftliche Veränderungen in der Hallstattzeit für den Niedergang der Almwirtschaft verantwortlich. Der florierende Salzhandel und der intensive Warenaustausch könnten Gründe sein, dass es zu keiner Neubelebung der Almwirtschaft auf dem Dachsteingebirge während der frühen und späten Eisenzeit gekommen ist.

\section{Dank}

Die archäologischen Feldforschungen wurden in Zusammenarbeit mit der ANISA, Verein für alpine Forschung, und dem Österreichischen Bundesdenkmalamt in Graz durchgeführt.

\section{Literatur}

Barth F. E. \& Reschreiter H. 2005. Fundbericht. Fundberichte aus Österreich 44: 482.

Kanelutti E. \& Rabeder G. 1990. Das archäozoologische Fundgut. In: Dachstein. Die Lackenmoosalm. Mitteilungen der ANISA 11: 200-202.

Mandl F. 1999. Schwaigenzeit ist Almenzeit. Über die Viehhaltung auf Almen des Dachsteins und Toten Gebirges im Mittelalter und in der Neuzeit. Beiträge zur Mittelalterarchäologie in Österreich. Österr. Ges. Mittelalterarchäologie 15: 83-98.

Mandl F. 2006 a. Dachsteinalmen. Gjaid und Taubenkar mit dem Hallstätter Gletschervorfeld. In: Alpen. Archäologie, Geschichte, Gletscherforschung. Mitteilungen der ANISA 25./26. Jg., 159171.

Mandl F. 2006 b. Almen und Salz. Hallstatts bronzezeitliche Dachsteinalmen. Jb. Oberösterr. Musealverein $151: 7-36$.

Mandl F. 2007. Die datierten bronzezeitlichen Hüttenreste auf dem östlichen Dachsteinplateau. Hallstatts bronzezeitliche Dachsteinalmen. Königreich-Alm Dachsteingebirge. 3500 Jahre Almwirtschaft zwischen Gröbming und Hallstatt. Forschungsber. ANISA 1: 74-81

Mandl F. 2008. Prähistorische Almwirtschaft. In: SALZ - REICH. 7000 Jahre Hallstatt. Hrsg. A. Kern, K. Kowarik, A. W. Rausch, H. Reschreiter. Veröffentl.Prähistorischen Abteilung (VPA) 2, 2008., 80f. 


\section{Klimawandel in Österreich}

Nicolussi K., Kaufmann M., Patzelt G., van der Pflicht J., Thurner A. 2005. Holocene treeline variability in the Kauner Valley, central Eastern Alps indicated by dendrochronological analysis of living trees and subfossil logs. Veget. Hist. Archaeobot. 14: 221-234.

Patzelt G. 2003. Forschungen im Gletscher Vorfeld. In: Almen im Herzen Österreichs Hrsg. v. ANISA, Verein für alpine Forschung. S. 184.

Schmidt R., Kamenik C., Roth M. 2006. Siliceous algae-based seasonal temperature inference and indicator pollen tracking ca. 4,000 years of climate/land use dependency in the southern Austrian Alps. J. Paleolimnol. 38: 541-554.

Stöller T. 1996. Neue Beiträge zur vorgeschichtlichen Besiedlung von Hallstatt. Jb. Oberösterr. Musealverein 141, $117 \mathrm{ff}$.

Tiefengraber S. 2007. Archäologische Untersuchungen in einer prähistorischen Almhütte im Königreich - Tiefkar. In: Königreich-Alm Dachsteingebirge. 3500 Jahre Almwirtschaft zwischen Gröbming und Hallstatt. Forschungsberichte ANISA 1: 97-108. 


\title{
Die Schwarzkiefer als Niederschlagsindikator für Ostösterreich
}

\author{
Michael Grabner ${ }^{1)}$ und Franz Holawe ${ }^{2)}$ \\ 1) Universität für Bodenkultur Wien, Wien, Österreich \\ 2) Universität Wien, Institut für Geographie und Regionalforschung, Wien, Österreich
}

\section{Abstract}

\section{The black pine as precipitation indicator for Eastern Austria}

The aim of the project was to find precipitation sensitive trees in Eastern Austria and to perform a 500 year long reconstruction of precipitation sums. The black pine (Pinus nigra var. austriaca) is described in the literature as a precipitation sensitive tree and due to its high age (up to 700 years) it is possible to set up 500 year long reconstructions of precipitation totals in the area of the "Thermenlinie" in the eastern part of Austria. As there is only a small number of trees available in the early period we focus on the period from 1700 to 1995 AD. In addition the parameter "false rings" (or density deviations) was used to reconstruct the amount of precipitation in May. This reconstruction shows interesting results throughout the period from 1880 to 1995 AD especially regarding the interpretation of year rings.

\section{Einleitung}

Ist man an einem Zeitfenster der letzten Jahrtausende interessiert, stehen z.B. Jahrringe als Proxy mit hoher zeitlicher Auflösung und exakter Datierbarkeit zur Verfügung. In vielen Datensätzen, welche die Temperaturentwicklung des letzten Jahrtausends beinhalten, sind daher vorwiegend Jahrringe eingeflossen (Mann et al, 1998, 1999). Die Mehrzahl der Nutzung von Jahrringen als Träger für Klimainformation hat die Rekonstruktion der Temperatur im Visier (D’Arrigo et al. 1999, Esper et al. 2002, Nicolussi 2008). Weniger häufig liegen Studien vor, in denen der Niederschlagsverlauf aus Jahrringen rekonstruiert wird (Watson \& Luckman 2001; Griggs et al. 2007). Das liegt an der großen räumlichen wie zeitlichen Variabilität dieses Klimaelements, steht aber auch in einem engen Zusammenhang mit dem Fehlen geeigneter Baumarten in klimatologisch geeigneter Umgebung. Im Osten Österreichs wurde im Rahmen des FWF - Projektes P9200-GEO die Schwarzkiefer als Proxy für den Niederschlag untersucht. Im Folgenden werden die wesentlichen Ergebnisse dieses Projektes (Zitate) kurz präsentiert und mit weiteren Analysen ergänzt. 


\section{Datengrundlage}

\section{Jahrringdaten}

Das Wachstum der Schwarzkiefer (Pinus nigra var. austriaca) wird hauptsächlich vom Niederschlag gesteuert (Strumia et al. 1997, Strumia 1999, Wimmer et al. 2000, Strumia 1999). Dafür wurden an 29 Standorten Bohrkernproben von jeweils 8 bis 20 Bäumen (in Summe 313 Bäume) genommen. Sowohl bei der Auswahl der Standorte als auch der Bäume wurden die lokalen Standortbedingungen berücksichtigt, bei der insbesondere die Wasserversorgung auf der Basis geologischer und pedologischer Unterlagen abgeschätzt worden ist (Strumia, 1999).

\section{Klimadaten}

Der Osten bzw. Nordosten Österreichs zeichnet sich durch geringe Jahresniederschlagssummen aus. Diese liegen im Weinviertel, in Teilen des Wiener Beckens und dem Burgenland zwischen 500 und $700 \mathrm{~mm}$ pro Jahr. Der Jahresgang ist deutlich ausgeprägt und weist typisch für diesen Klimatyp ein Niederschlagsmaximum während des Sommers auf. Entscheidend für die sprichwörtliche Trockenheit ist die Konzentration des sommerlichen Niederschlags auf wenige Einzelereignisse großer Intensität und die temperaturbedingte hohe Verdunstung. Aber auch die Trockenperioden dauern hier länger und sind häufiger anzutreffen, als im vergleichsweise feuchten Alpenvorland (Nobilis, 1985). Die Standorte der Schwarzkiefern liegen hauptsächlich im Bereich des Überganges der Alpen ins Wiener Becken, ein Gebiet, welches von der klimatischen Trockengrenze durchzogen wird, entsprechend gering positiv oder negativ ist ihre klimatische Wasserbilanz (Harlfinger 1999, BMLFUW 2005).

Obwohl Strumia (1999) für seine Analysen die Klimadaten mehrerer Stationen verwendete, wurden die Berechnungen für die vorliegende Darstellung ausschließlich mit den Daten von Wien - Hohe Warte durchgeführt. Von dieser Station liegen ab 1841 homogenisierte Klimadaten auf Monatsbasis vor (Auer et al. 2001), von denen die Niederschlagssummen in dieser Untersuchung herangezogen wurden.

\section{Methoden}

Die Jahrringzeitreihen wurden zunächst mit verschiedenen Methoden vom Alterstrend befreit, um die nicht klimatischen Faktoren im Wachstum möglichst zu eliminieren. Für diese Indexierung oder Standardisierung genannte Beseitigung des Alters- 
trends gibt es keine allgemein gültige Methode (Fritts 1976). Es ist daher zunächst in jedem Falle notwendig, die jeweils geeignete Methode der Indexierung herauszufinden. Im gegenständlichen Fall erwiesen sich die Modellierung des Alterstrends mit einem Spline bzw. durch eine regionale Kurven-Approximation (Zitat) mit nachfolgender autoregressiver Anpassung als beste Lösungen für dieses Problem. Zudem wurden neben Mittelwerten und Standardabweichungen die bei der Auswertung der Jahrringsequenzen üblichen Kenngrößen, wie das Signal zu Rauschverhältnis oder die Signal-Stärke, als Funktion des Stichprobenumfanges berechnet.

Der Zusammenhang der indexierten Reihen mit den Zeitreihen der Klimavariablen erfolgt über eine multiple Regressionsanalyse. Dabei erfolgt diese Analyse nicht mit den Klimavariablen, sondern mit den aus ihnen extrahierten Hauptkomponenten (von Storch und Zwiers 1999). Das hat den Vorteil, dass Kollinearitäten ausgeschaltet werden, da die Prediktoren orthogonal und deren Zeitkoeffizienten nicht korreliert sind. Damit lässt sich eine Regressionsgleichung aufstellen, mit deren Hilfe aus dem Verlauf der indexierten Ringbreiten oder, im gegenständlichen Fall, Spätholzanteile, der Verlauf der Klimavariable, hier die Summe des Sommerniederschlags, rekonstruiert wird. Dabei werden die Parameter der Regressionsgleichung mittels Bootstrap-Methode geschätzt. In der Regressionsgleichung, mit deren Hilfe die Niederschlagssumme des Mai geschätzt wird, steckt als Dummy-Variable die Häufigkeit der in den Jahrringen festgestellten Dichteschwankungen. Dabei wird ein Schwellwert von 0,4 (Einheit/Häufigkeit?) verwendet und damit die Zeitreihe der Dichteschwankungen binär kodiert.

Bei den verwendeten Niederschlagsdaten handelt es sich um die bereits homogenisierten Zeitreihen der Station Wien Hohe Warte (Auer et al. 2001). Für die Berechnungen im Rahmen dieser Untersuchung wurden die auf den Zeitraum 19011990 bezogenen Abweichungen herangezogen, wohingegen sich Strumia (1999) auf den Zeitraum 1961-1990 bezog. Uns schien eine längere Bezugsperiode die bessere Wahl zu sein, auch wenn damit die Vergleichbarkeit mit den Ergebnissen anderer Studien (z.B. Casty et al. 2005) erschwert wird. Für die Analyse des Zusammenhanges der Dichteschwankungen mit den Niederschlagssummen der Monate April bis Juni wurden die Messdaten binär kodiert. Dabei diente der Median als Schwellwert. Demgemäß gibt es in dieser neuen Zeitreihe nur zwei Zustände, feucht und trocken. 
Klimawandel in Österreich

\section{Ergebnisse}

Bereits in einer ersten Darstellung vorläufiger Ergebnisse wurde ein deutlicher Zusammenhang zwischen dem Zuwachs und dem Niederschlag der Monate April bis August aufgezeigt. Zudem wiesen einige der Bäume ein Alter von mehreren hundert Jahren auf, sodass eine Rekonstruktion des Niederschlags über einen längeren Zeitraum möglich schien (Strumia et al. 1997). In einer weitführenden und sehr detaillierten Analyse stellte Strumia (1999) das Potential der Schwarzkiefer als Klimaindikator dar. Nach einer umfassenden Präsentation der Grundlagen der Dendrochronologie finden sich in dieser Untersuchung viele Details der Niederschlags-Wachstums Beziehung der Schwarzkiefer. Die räumliche Verteilung der Probenstandorte erlaubte es, räumliche Unterschiede herauszufiltern, den Verlauf des Niederschlags sowohl für die Sommermonate (JJA?) als auch das Zeitfenster von April bis August zu rekonstruieren. Auf den Zusammenhang der Dichteschwankungen mit der Trockenheit des Mai wird bereits in Strumia (1999) hingewiesen. Eine detaillierte Auswertung dieses Zusammenhanges erfolgte in einer weiteren Untersuchung (Wimmer et al. 2000).

\section{Rekonstruktion der Niederschlagssumme von Juni bis August im Zeitfenster von 1700 bis 1845}

Aus seinen Analysen folgerte Strumia (1999), dass wegen der zu geringen Belegungsdichte vor 1630 Aussagen über den abgeleiteten Niederschlagsverlauf zu unsicher wären und deshalb eine zeitliche Einschränkung auf den Zeitbereich nach 1630 notwendig wäre. Obwohl Strumia (1999) den Zeitraum ab 1630 als im statistischen Sinne gut belegt ansieht, gibt es im Zeitbereich von 1630 bis ca. 1700 noch zu untersuchende Unklarheiten in den Daten, sodass in der hier vorgelegten Zusammenstellung der Zeitraum ab 1700 zur Darstellung gelangt. Zudem wird der Niederschlag auf das Zeitfenster von Juni bis August, also die Sommermonate eingeschränkt. Für die Rekonstruktion wurde in dieser Analyse außerdem nur der Spätholzanteil als Prädiktor verwendet, wobei sich die dargestellten Abweichungen auf den Zeitraum 1901-1990 beziehen. Die Verwendung des Spätholzanteils erlaubt es, je nach dem zugrundeliegenden Zeitraum, ca. 36 bis 50\% der Varianz der Niederschlagssumme der Sommermonate zu erklären (Tab. 1). Es wurde zunächst eine Zweiteilung der Zeitreihe des Sommerniederschlags vorgenommen, jeweils ein Modell für beide Zeiträume kalibriert und anschließend im jeweils anderen Zeitraum verifiziert. Aufgrund der Ergebnisse dieser Analyse (Tab. 1) hätte man im Prinzip jedes dieser 
vier Modelle für die Rekonstruktion verwenden können. Der Bezug auf den Kalibrationszeitraum 1846-1920 ist in beiden Fällen etwas besser geeignet als der Zeitraum 1921-1995. Deswegen wurde letztendlich dem Modell 1 der Vorzug gegeben. Der offensichtlich geringere Zusammenhang zwischen Niederschlag und Zuwachs im Zeitfenster 1921 bis 1995 ist wohl eine Folge der insbesondere ab den 1950er Jahren merkbaren Entkopplung dieses Zusammenhanges (Strumia et al. 1997, Brazdil et al. 2001, Wilson et al. 2005).

Zur Darstellung gebracht wurde der Sommerniederschlag (Abb. 1), der mittels des Spätholzanteiles für den Zeitraum ab 1700 als Abweichung von 1901-1990 rekonstruiert wurde (Gleichung 1). Es ist letztlich im Falle der linearen Regression bloß eine Umkodierung. Aus dem Verlauf lässt sich kein Trend ableiten, wohl aber in der Spektralanalyse der Daten (nicht dargestellt) mehrere deutlich ausgeprägte Perioden. Neben mehreren kürzeren Perioden, sind dabei eine 15, 18, 28 und 47- jährige Periode hervorzuheben. Der besseren Übersichtlichkeit und Einheitlichkeit wegen sind sowohl die gemessenen Niederschlagssummen als auch die rekonstruierten Werte mit einem 15-jährigen Tiefpassfilter geglättet worden (Abb. 1).

Tabelle 1: Der Zusammenhang zwischen dem Spätholzanteil und dem Sommer - Niederschlag für zwei Methoden der Bereinigung des Alterstrends und zwei Bezugszeiträume $\left(S H_{S T D}\right.$, Spätholzanteil nach der Standardisierung;

$\mathrm{SH}_{\mathrm{RES}}$, Spätholzanteil nach der AR-Anpassung; 01-90, Abweichungen bezogen auf den Zeitraum 1901-1990)

\begin{tabular}{cccccc}
\hline Modell & SH $_{\text {STD }}$ & Kalibration & $\mathbf{r}^{\mathbf{2}}$ & Verifikation & $\mathbf{r}^{\mathbf{2}}$ \\
\hline 1 & $01-90$ & $1846-1920$ & 0,5052 & $1921-1995$ & 0,3628 \\
2 & $01-90$ & $1921-1995$ & 0,3674 & $1846-1920$ & 0,4997 \\
& $\mathbf{S H}_{\text {RES }}$ & & & & \\
3 & $01-90$ & $1846-1920$ & 0,4958 & $1921-1995$ & 0,4173 \\
4 & $01-90$ & $1921-1995$ & 0,3679 & $1846-1920$ & 0,4938 \\
\hline
\end{tabular}

Gleichung 1: Die lineare Regressionsbeziehung zwischen Sommerniederschlag und Spätholzanteil (ausgedrückt als Abweichungen von 1901-1990; $S H_{S T D}$, Spätholzanteil nach der Standardisierung).

$$
\mathrm{NS}_{\text {Som }}=-288.95+291.19^{*} \mathrm{SH}_{\text {STD }}
$$

Im Zeitfenster, in dem auch Messwerte vorliegen, zeigt sich, abgesehen von der Amplitude und einer Phasenverschiebung im späten 19. Jahrhundert, eine grund- 
sätzlich gute Übereinstimmung mit den vom Modell geschätzten Werten. Besonders gut wird der Verlauf in der zweiten Hälfte des 19. Jahrhunderts und im gesamten 20. Jahrhundert wiedergegeben. Vertraut man auf die Güte des Modells auch in der Vergangenheit, dann lassen sich feuchtere Phasen, z.B. am Anfang, gegen die Mitte und in der Mitte der 2. Hälfte des 18. Jahrhunderts ausmachen (siehe Abb. 1). Von den trockenen Phasen sind vor allem Zeitabschnitte am Anfang und gegen die Mitte des 19. Jahrhunderts hervorzuheben. In der betrachteten Zeitreihe ist eine ausgeprägte Abfolge von Jahren mit feuchten und trockenen Sommermonaten enthalten. Einen sehr ähnlichen Verlauf hat Strumia (1999) mit einem anderen Modell und einem anderen Bezugszeitraum, aber unter Verwendung der gleichen Daten erhalten. Ein visueller Vergleich mit der Zusammenstellung in Casty et al. (2005) führt nur in Ausnahmen zu einer Übereinstimmung. Auf eine detaillierte Analyse wird hier jedoch mangels Vergleichsdaten verzichtet.

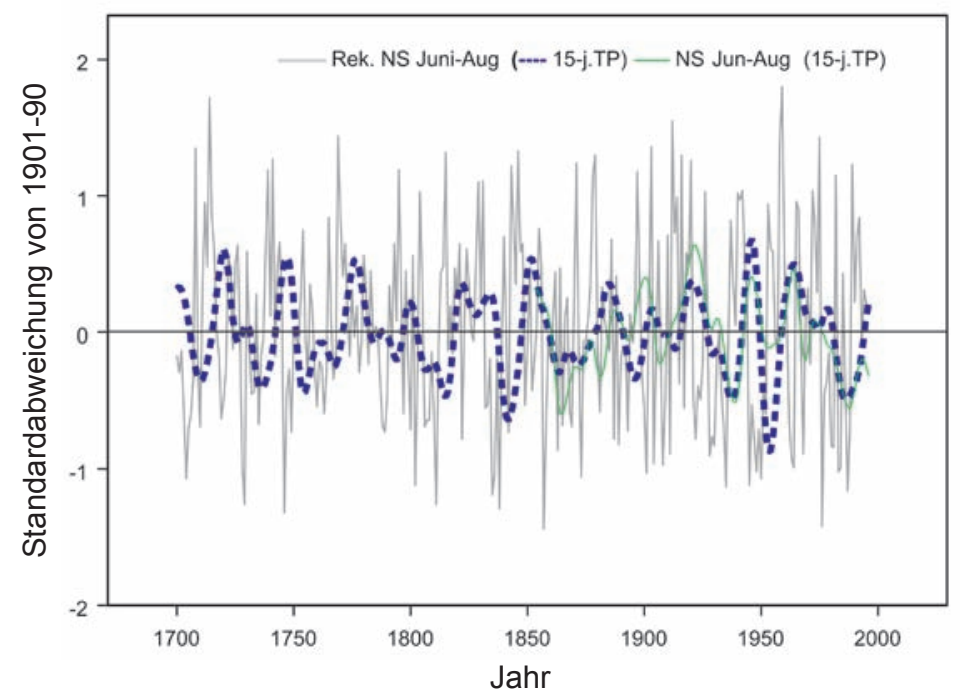

Abb. 1: Der Verlauf des Sommerniederschlags im Zeiffenster 1700-1995 (ausgedrückt als Abweichungen von 1901-1990)

Fig. 1: Time course of summer precipitation between 1700 and 1995 epressed as deviation from the mean 1901-1990.

\section{Rekonstruktion des Frühsommerniederschlags mittels Dichteschwankungen}

Im Zuge der Untersuchungen wurde die Eignung so genannter falscher Jahrringe der Schwarzkiefer, auch Dichteschwankungen genannt, für die Klimarekonstruktion überprüft. Es stellte sich zunächst heraus, dass es dabei eine Altersabhängigkeit gibt und jüngere Bäume mit größerer Häufigkeit derartige Signaturen ausbilden (Strumia, 1999). In einer weiteren Untersuchung (Zitat) konnte ein Zusammenhang mit der Trockenheit des Mai gezeigt werden. Dabei standen 50\% der Jahre mit einem erhöhten Auftreten der Dichteschwankungen mit einem feuchten April, einem trockenen Mai und einem eher feuchten Juni im Zusammenhang (Wimmer et al. 2000). 
Auf einen möglichen Zusammenhang mit dem Klima hat Strumia (1999) bereits hingewiesen, dieses Phänomen jedoch nicht sehr ausführlich diskutiert. In mehreren Untersuchungen wurden ,falsche Jahrringe“ als Indikatoren für das Klima verwendet (Priya and Bhat 1998, Hoffer 2007). Neben anderen Umweltfaktoren scheint die Frühsommertrockenheit oder eine Kombination aus trockenem Frühjahr und feuchtem Sommer eine gewisse Rolle bei der Ausprägung dieser Merkmale zu spielen (Villalba \& Veblen 1996, Hoffer 2007). Es wurde eine Abhängigkeit der Häufigkeit des Auftretens von Dichteschwankungen vom Alter der Bäume festgestellt. Da die Schwarzkiefer offensichtlich in jüngeren Jahren häufiger falsche Jahrringe ausbildet, mussten alle Bäume unter 100 Jahren von dieser Untersuchung ausgeschlossen werden. Dementsprechend standen die Dichteschwankungen nur bis zum Jahre 1880 zur Verfügung.

Trockenheit im Mai begünstigt die Ausbildung der Dichteschwankungen. Im Zeitraum von 1880-1995 weisen jene Jahre, in denen keine oder eine geringe Zahl von Dichteschwankungen festzustellen ist, nur zu 28\% einen trockenen Mai auf. Im Gegensatz dazu war der Mai in 75\% der Fälle trocken, wenn die Häufigkeit der Dichteschwankungen größer als der Median des Gesamtzeitraumes ausfiel. Benutzt man jenen Schwellwert der Häufigkeit der Dichteschwankungen, der sich in einer Regressionsuntersuchung als der am besten geeignete herausstellte, kommt die Bedeutung eines trockenen Monats Mai noch deutlicher zum Ausdruck. Während in 36\% jener Jahre unterhalb dieses Schwellwertes der Mai trocken (und demnach in 64\% feucht) gewesen ist, so ist das in 90\% der Jahre der Fall, in denen dieser Schwellwert überschritten wurde. Zudem lassen sich Kombinationen von feuchten und trockenen Monaten ausmachen, welche offensichtlich die Bildung derartiger Merkmale begünstigen (Tabelle 2). Ein trockener Mai in Kombination mit einem feuchten Juni führt zu hohen Dichteschwankungen, wenn auch der April noch feucht war, nimmt die Anzahl der Dichteschwankungen um das Zweieinhalbfache zu, aber ein feuchter April führt in Kombination mit einem trockenen Mai ebenfalls zu erhöhten Dichteschwankungen, auch bei trockenem Juni. Ein feuchter Mai

Tabelle 2.: Die Häufigkeit der des Auftretens von Dichteschwankungen abhängig von der Kombinationen von trockenen und feuchten Monaten im Zeitraum 1880-1995. 0 bedeutet trocken, 1 feucht. Die erste Stelle stellt den April dar; die zweite Mai und die dritte Juni. Somit bedentet ₹.B. 101: feuchter April, trockener Mai und feuchter Juni; LADF, Häufigkeit der Dichteschwankungen

\begin{tabular}{cccccccccc} 
& 000 & 001 & 010 & 100 & 011 & 101 & 110 & 111 & $\sum$ \\
\hline IADF $\leq 0,4$ & 14,0 & 4,6 & 20,9 & 11,7 & 16,2 & 5,9 & 10,4 & 16,3 & 100,0 \\
IADF $>0,4$ & 6,7 & 20,0 & 6,7 & 13,3 & 0,0 & 50,0 & 3,3 & 0,0 & 100,0 \\
\hline
\end{tabular}


jedoch oder drei trockene Monate (AMJ) führen zu keinen oder geringen Dichteschwankungen.

Die Häufigkeit der Dichteschwankungen (IADF) lässt sich aber auch als Variable in eine Regressionsgleichung einbeziehen (Gleichung 2). Dabei hat sich ein Schwellenwert der Häufigkeit der Dichteschwankungen von 0,4 als optimal erwiesen. In Kombination mit der Frühholzbreite lassen sich damit etwa 35\% der Varianz der Niederschlagssumme des Mai erklären (Tab. 3).

Tabelle 3: Der Zusammenhang zwischen dem Niederschlag des Mai und einer Kombination aus Frühbolzbreite und den Dichteschwankungen als Dummy Variable für zwei Bezugszeiträume (0190.... Abweichungen bezogen auf den Zeitraum 1901-1990)

\begin{tabular}{cccccc}
\hline Modell & & Kalibration & $\mathbf{r}^{2}$ & Verifikation & $\mathbf{r}^{\mathbf{2}}$ \\
\hline 1 & $01-90$ & $1880-1937$ & 0,3434 & $1938-1995$ & 0,3919 \\
2 & $01-90$ & $1938-1995$ & 0,3723 & $1880-1937$ & 0,3769 \\
\hline
\end{tabular}

Gleichung 2: Die Regressionsbęiehung zwischen der Niederschlagssumme des Mai (ausgedrückt als Abweichungen von 1901-1990) und einer Kombination aus Frübholzbreite (=FH) und den Dichteschwankungen (=DSchw)als Dummy Variable.

$$
\mathrm{NS}_{\text {Som }}=-198.3-94.0^{*} \mathrm{D}_{\text {Schw }}+233.79^{*} \mathrm{FH}_{\text {STD }}
$$

Die mittels der Dichteschwankungen erzielten Ergebnisse sind viel versprechend. Zwar gelingt es nicht, jede Entwicklung des Mainiederschlags in den Jahrringen nachzuvollziehen, aber abgesehen von weniger deutlich ausgeprägten Extremen

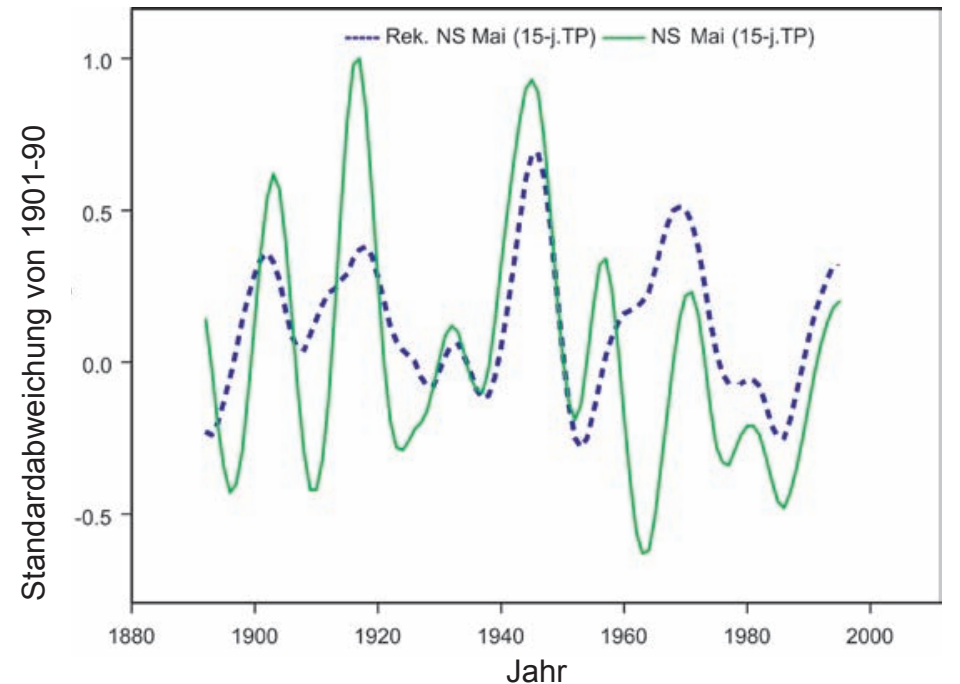

Abb. 2: Der Verlauf des Niederschlags des Mai im Zeitfenster 1890-1995 (ausgedrückt als Abweichungen von 1901-1990)

Fig. 2: Time course of precipation during May between 1890 and 1995 expressed as deviation from the mean 1901-1990. 
und einer deutlichen Abweichung im Zeitfenster von 1960 bis 1970 stimmt aber der Verlauf der aus den Jahrringen rekonstruierten Niederschlagssummen gut mit dem Verlauf der gemessenen Werte überein. Das lässt sich am besten bei den gefilterten Zeitreihen erkennen (Abb. 2).

\section{Schlussfolgerungen}

Die bisher vorliegenden Untersuchungen (Strumia et al. 1997, Strumia 1999, Wimmer et al. 2000) haben das Potential der Schwarzkiefer für die Rekonstruktion des Niederschlags ausgewählter Zeitfenster aufgezeigt. Mit einer Verdichtung der Belegung im Zeitbereich vor 1700 ließe sich der Zeitraum zumindest um mehrere hundert Jahre verlängern.

\section{Dank}

Wir bedanken uns beim FWF für die Finanzierung des Projekts P9200-GEO.

\section{Literatur}

Auer I., Böhm R., Schöner W. 2001: Austrian long-term Climate 1767-2000. (ALOCLIM) Multiple Instrumental Climate Time Series from Central Europe. Österreichische Beiträge zu Meteorologie und Geophysik 25, Zentralanstalt für Meteorologie und Geodynamik, Wien 397: 147p+Data CRRom.

BMLFUW (Hrsg.) 2005: Hydrologischer Atlas Österreichs. 2. Lieferung. Bundesministerium für Land- und Forstwirtschaft, Umwelt und Wasserwirtschaft. ISBN 3-85437-250-7, Wien

Brázdil R., Stepankova P., Kyncl T., Kyncl J. 2002: Fir tree-ring reconstruction of March-July precipitation in Southern Moravia (Czech Republic), 1376-1996. Climate Research, 20, 2002, 223 239.

Brázdil R., Pfister C., Wanner H., v. Storch H., Luterbacher J. 2005: Historical Climatology in Europe - the state of the art. Climatic Change 70, 363-430.

Casty C., Wanner H., Luterbacher J., Esper J., Böhm R. 2005: Temperature and precipitation variability in the European Alps since 1500. Int. J. Climatol. 25: 1855-1880.

D’Arrigo R., Jacoby G., Free M., Robock A. 1999: Northern Hemisphere temperature variability for the past three centuries: tree-ring and model estimates. Climatic Change 42, 663-675.

Esper J.; Cook E.R., Schweingruber F.H. 2002: Low-frequency signals in long tree-ring chronologies for reconstructing past temperature variability. Science, 295, 2250-2253.

Fritts H.C. 1976: Tree rings and climate. Academic Press, London, New York, San Francisco, 567 pp.

Glaser R., Brázdil R., Pfister C., Dobrovolny P., Barriendos Valvé M., Bokwa A., Camuffo D., Kotyza O., Limankówka D., Rácz L., Rodrigo F.S. 1999: Seasonal temperature and precipitation fluctuations in selected parts of Europe during the sixteenth century. Climatic Change, 43, 169-200. 


\section{Klimawandel in Österreich}

Griggs C., Degaetano A., Kuniholm P., Newton M. 2007: A regional high-frequency reconstruction of May-June precipitation in the north Aegean from oak tree rings, A.D. 1089-1989. Int. J. Climatol. 27: 1075-1089.

Harlfinger O., Knees G. 1999: Klimahandbuch der Österreichischen Bodenschätzung. Mitteilungen der Österreichischen Bodenkundlichen Gesellschaft, 58, 196 S.

Hoffer M. 2007: Density fluctuations (false rings) in jack pine and black spruce trees, Nopiming Provincial Park, Manitoba. Thesis, Dept. of Biology, University of Manitoba, 52 p.

Hughes M.K. 2002: Dendrochronology in climatology - the state oft he art. Dendrochronologia 20/1-2, 95-116.

Mann M.E., Bradley R.S., Hughes M.K. 1998: Global-scale temperature patterns and climate forcing over the past six centuries. Nature, 392, 779-787.

Mann M.E., Bradley R.S., Hughes M.K. 1999: Northern hemispheric temperatures during the past millennium: inferences, uncertainties, and limitations. Geophysical Research Letters 26: 759-762.

Matulla C., Penlap E.K., Haas P., Formayer H. 2003: Comparative analysis of spatial and seasonal variability: Austrian precipitation during the 20th century. Int. J. Climato. 23, 1577-1588.

New M., Todd M., Hulme M., Jones P. 2001: Precipitation measurements and trends in the twentieth century. Int. J. Climato. 21, 1899-1922.

Nicolussi, K. (2008) Alpine Dendrochronologie - Untersuchungen zur Kenntnis der holozänen Umwelt- und Klimaentwicklung. In: alpine space - man \& environment 6, 41-54.

Nobilis F. 1985: Trockenperioden in Österreich. Verband der Wissenschaftlichen Gesellschaften Österreichs, Vienna, 293 p.

Priya P.B., Bhat K.M. 1998: False ring formation in teak (Tectona grandis L.f.) and the influence of environmental factors. Forest Ecology and Management 108, 215-222.

Schönwiese C.D. 2003: Klimatologie, UTB Stuttgart, 440.S

Strumia G., Wimmer R., Grabner M. 1997: Dendroclimatic Sensitivity of Pinus Nigra Arnold in Austria. Dendrochronologia, 15, 129-137,

Strumia G. 1999: Tree-ring based reconstrution of precipitation in Eastern Austria. Dissertation at the University of Life Sciences, Vienna, Austria, 113 p.

Villalba R., Veblen T.T. 1996: A tree-ring record of dry spring - wet summer events in the foreststeppe ecotone, northern Patagonia, Argentinia. In: Tree rings, Environment and Humanity: Proceedings of the International Conference, 17-21 May 1994, Tucson, Arizona. Edited by J.S.Dean, D.M. Meko, and T.W. Swetnam. Department of Geosciences, University of Arizona, Tucson, 107-116.

Villalba R., Cook E.R., Jacoby G.C., D’Arrigo R., Veblen T.T., Jones P.D. 1998: Tree-ring based reconstructions of northern Patagonia precipitation since AD 1600. The Holocene 8, 6, 659674.

Watson E., Luckman B.H. 2001: Dendroclimatic reconstruction of precipitation for sites in the southern Canadian Rockies. The Holocene 11, 2, 203-213.

Wilson R.J.S, Luckman B.H., Esper J. 2005: A 500 year dendroclimatic reconstruction of spring -summer precipitation from the Lower Bavarian Forest region, Germany. Int. J. Climatol. 25, 611-630.

Wimmer R., Strumia G., Holawe F. 2000: Use of false rings in Austrian pine to recostruct early growing season precipitation. Can. J. For. Res. 30: 1691-1697 


\title{
Die Sedimentablagerungen des Mondsees (Ober- österreich) als ein Archiv extremer Abflussereignisse der letzten 100 Jahre
}

\author{
T. Swierczynski, S. Lauterbach, P. Dulski, A. Brauer \\ Deutsches GeoForschungsZentrum Potsdam, Sektion 5.2 - Klimadynamik und \\ Landschaftsentwicklung, Potsdam, Deutschland
}

\section{Abstract}

Sediment deposits of Lake Mondsee (Upper Austria) as an archive for extreme runoff events during the past 100 years

11 short cores from Lake Mondsee reveal a continuous sedimentation history of the last 100 years. The annually laminated sediments enabled the reconstruction of lake sedimentation in a seasonal resolution. 12 detrital layers were identified in the lake sediments. 9 corresponding floods and one debris flow in 1986 were verified by historical archives and hydroclimatological datasets. Two layers with significant sedimentological and geochemical signatures suggest two more debris flows in 1923 and 1941, for which historical evidence still has to be proved.

\section{Einleitung}

In der derzeitig geführten Klimadebatte rücken Extremereignisse in Verbindung mit außergewöhnlich hohen Niederschlägen, wie beispielsweise das Sommerhochwasser im Jahr 2002, zunehmend in den Mittelpunkt des Interesses. Die statistische Auswertung von Abflusssituationen der mitteleuropäischen Flüsse Elbe und Oder konnte für die letzten 80-150 Jahre jedoch keine Zunahme der Hochwasserhäufigkeit feststellen, für das Auftreten von Winterhochwässern sogar abnehmende Tendenzen (Mudelsee, 2003). Grundlage für die verlässliche statistische Auswertung der Auftretenshäufigkeit von Hochwasserereignissen ist die Verfügbarkeit von Aufzeichnungen über Zeitpunkt und Stärke dieser Ereignisse. Instrumentelle Daten (z.B. Pegelstände, Abflussmengen) umfassen meist nur einen Zeitraum von 100-150 Jahren. Historische Quellen (z.B. Chroniken, Hochwassermarken) lassen sich zwar weiter zurückverfolgen, der Bemessungsgrad von Hochwasserereignissen erfolgt allerdings anhand des Überschwemmungsschadens und ist außerdem stark von der subjektiven Einschätzung des Quellenverfassers geprägt. Die bisherigen Zeitreihen ermöglichen deshalb keine zuverlässigen Aussagen zur längerfristigen Häufigkeit von Hochwasserereignissen. 
Im Gegensatz dazu bieten Sedimentarchive, wie z.B. Seen, länger zurückreichende Zeitreihen mit wichtigen umweltrelevanten Informationen. Der Vorteil von jahresgeschichteten Seesedimenten besteht neben einer verlängerten Zeitreihe vor allem in der Möglichkeit der Erstellung einer Chronologie für die in den Sedimenten aufgezeichneten Hochwasserereignisse. Während extremer Abflussereignisse gelangt das erodierte Material aus dem Einzugsgebiet durch die Zuflüsse in die „Sedimentfalle See“ und wird dort, eingeschaltet als charakteristische grobkörnige Eintragslage in den jahresgeschichteten Seesedimenten, abgelagert. Derzeit sind die konkreten Zusammenhänge von Hochwasserereignissen und Sedimenttransfer in den See jedoch noch unklar. Zur Untersuchung dieser Zusammenhänge bietet der Mondsee (Oberösterreich) ein gutes Sedimentarchiv, da (1) lange hydroklimatische Messreihen zu Niederschlägen und Abflussmengen im Einzugsgebiet sowie Pegelstände für die letzten 100 Jahre zur Verfügung stehen, und (2) die Jahresschichtung der Ablagerungen eine genaue Altersbestimmung für die Hochwasserereignisse innerhalb des Sedimentprofils erlaubt. Da zudem die Zuflüsse geologisch unterschiedliche Einzugsgebiete durchfließen, können die charakteristischen Sedimenteinträge und deren Verteilung im Seebecken gut rekonstruiert werden. Die bisherigen Untersuchungen unter speziellen sedimentologischen Fragestellungen (Horsthemke, 1986; Helbig, 1987; Wetzel, 1988; Herrmann, 1990) und limnologischen Aspekten (Jagsch et al., 1982; Schmidt et al., 1985, Dokulil \& Skolaut 1986, Klee \& Schmidt 1987, Dokulil et al. 2006) zeigen, neben einer sehr guten Datenlage, das umfangreiche Forschungspotenzial des Sees für paläoklimatische Studien auf.

In dieser Studie werden Kurzkerne aus unterschiedlichen Stellen im Seebecken vorgestellt. Anhand von Eintragslagen in den Seesedimenten werden im Vergleich mit instrumentellen Daten und historischen Aufzeichnungen die extremen Hochwasserereignisse der letzten 100 Jahre untersucht.

\section{Untersuchungsgebiet}

Der Mondsee befindet sich im nördlichen Alpengebiet Oberösterreichs, ca. $40 \mathrm{~km}$ östlich von Salzburg. Auf einer Höhe von $481 \mathrm{~m}$ ü. A. erstreckt sich der See mit einer Fläche von 14,2 $\mathrm{km}^{2}$ auf der geologischen Überschiebungszone zwischen dem im Norden des Sees anstehenden Flysch und dem Hauptdolomit der Nördlichen Kalkalpen am Südufer des Sees (Abb. 1). Der See kann in ein tieferes Südbecken und ein flacheres Nordbecken unterteilt werden. Das gesamte Einzugsgebiet von ca. $247 \mathrm{~km}^{2}$ entwässert mit den drei Hauptzuflüssen und weiteren kleinen Bächen in das 
Nordbecken, mehrere kleinere Wildbäche wie der Kienbach speisen das Südbecken. Unter limnologischen Aspekten wird der See derzeit als nährstoffarm (oligo/ mesotroph) eingestuft, allerdings konnten für den Zeitraum von 1968 bis 1983 zeitweise nährstoffreichere (eutrophe) Bedingungen festgestellt werden (Dokulil \& Skolaut 1986, Schmidt et al. 1985, Klee \& Schmidt, 1987).

Abb. 1: Vereinfacbte geologische Karte des Mondsees und Lage der Bobrlokationen (Kurzkerne). Detailanalysen (₹.B. ${ }^{137} \mathrm{Cs}$-Datierung und Elementanalysen) wurden am Kurvkern Mo_05_P3 vorgenommen.

Fig. 1: Simplified geological map of the area around Lake Mondsee and coring sites. Detailed analyses $\left({ }^{137} \mathrm{Cs}\right.$ and $\mu$-XRF element scanning) have been carried out at core Mo_05_ P3.

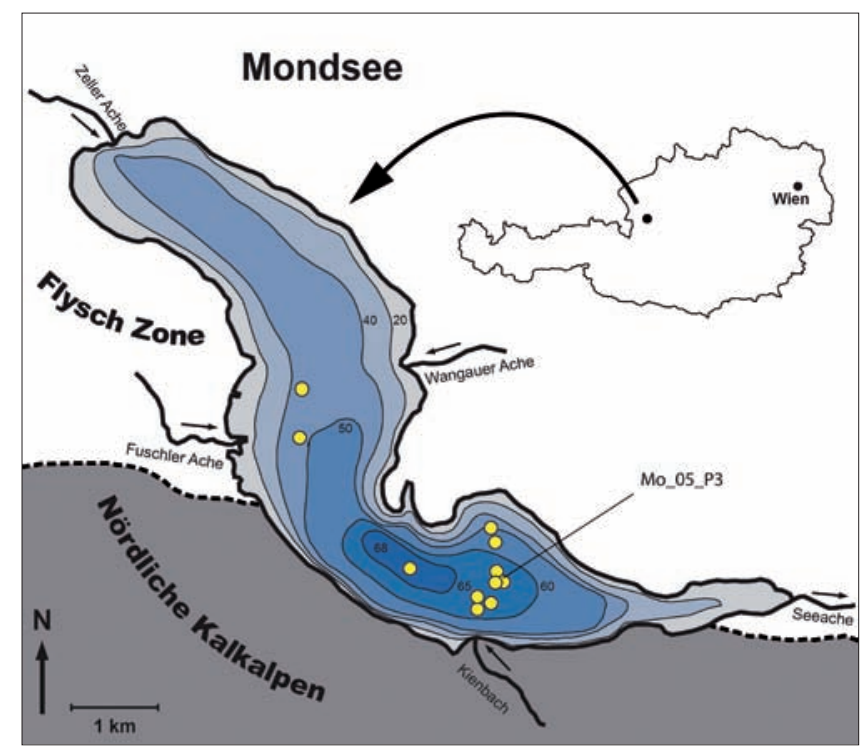

\section{Methoden}

Schwerpunkt dieser Studie war speziell die Untersuchung der Sedimenteinträge durch den Kienbach in das südliche Teilbecken des Sees. Zu diesem Zweck wurde ein Transekt von Bohrkernen mit zunehmendem Abstand vom Mündungsbereich des Kienbachs entnommen und das Sedimentationsverhalten vom Eintragsort über die Seemitte bis zum gegenüberliegenden Ufer hin untersucht. Zum Vergleich wurden zwei zusätzliche Kurzkerne aus dem nördlichen Seebecken entnommen, um die Sedimentation im Bereich der Mündung der Fuschler Ache zu untersuchen. Außerdem wurden zwei Bohrkerne aus dem tiefsten Bereich des Sees analysiert. Die Kurzkerne haben eine Länge von 43-104 cm. Alle Sedimentkerne wurden mit einem, von einem Boot heraus an einer Seilwinde operierenden, Niederreiter-Kurzkernsystem (UWITEC) gewonnen. Nach dem Öffnen der Kurzkerne im Labor wurden, nach der ursprünglich von Merkt (1971) beschriebenen Methode, $10 \mathrm{~cm}$ lange überlappende Sedimentabschnitte entnommen, gefriergetrocknet, mit Kunstharz 
vakuumimprägniert und schließlich zu feinen Scheiben, den sogenannten SedimentDünnschliffen, geschnitten. Neben der mikroskopischen Dünnschliff-Analyse der Seesedimente liefern geochemische Untersuchungen weitere nützliche Informationen über die Zusammensetzung der Sedimente sowie die Herkunftsgebiete des eingetragenen Materials.

\section{Altersbestimmung}

Unter bestimmten Bedingungen kann es in Seen in jedem Jahr zur Ausbildung charakteristischer Ablagerungen (Warven) kommen, welche jahreszeitlich eine unterschiedliche Zusammensetzung aus seeeigenem (endogenem) und eingetragenem Material aufweisen (Brauer, 2004). Die Auszählung der Lagenpaare erlaubt im Falle der festgestellten Jahreszeitlichkeit die Erstellung einer durchgängigen und unabhängigen Chronologie. Der Jahrescharakter der laminierten Sedimente des Mondsees konnte durch zwei Methoden bestätigt werden: (1) In der Sedimentabfolge des Kurzkerns Mo_05_P3 lassen sich zwischen 1985 und 2005 exakt 20 zählbare Jahreslagen nachweisen. Die markante Jahreslage 1985 diente als ein Referenzjahr, da dieses Jahr die ehemals oberste Lage eines Bohrkern aus dem Jahr 1985 bildete. (2) Einen weiteren Beweis lieferte eine unabhängige Datierung der Sedimente mittels der spezifischen Aktivität des radioaktiven Isotops Caesium-137 (Abb. 2), welche exemplarisch am Kurzkern Mo_05_P3 vorgenommen wurde. Sogenannte „fall-outpeaks“, also Höhepunkte in der Belastung der Umwelt mit Caesium-137, in den

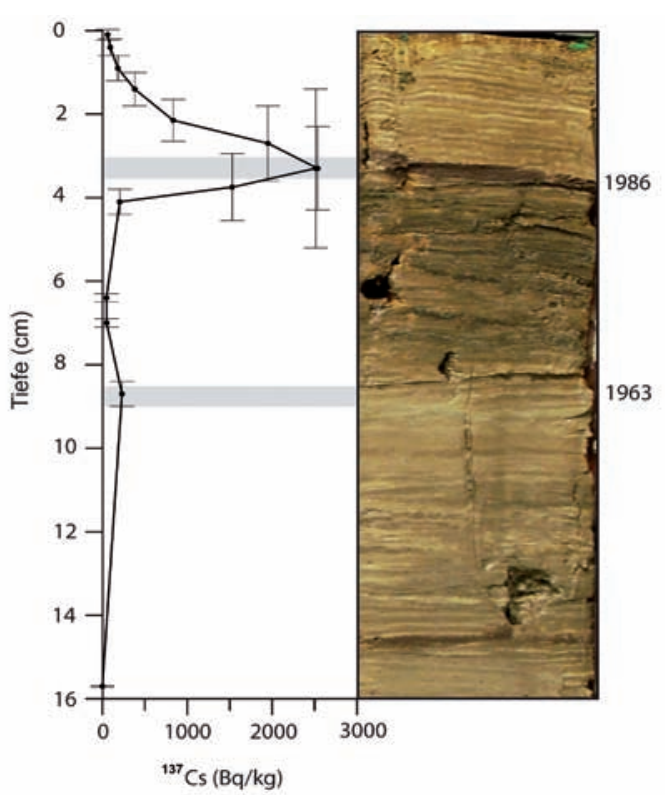

Abb. 2: ${ }^{137}$ Cs-Datierung (J. -L. Reyss) am Kurakern Mo 05_P3 mit Spitzenwerten in der Radioaktivität durch den nuklearen Fallout in den Jahren 1963 (Atomwaffentests) und 1986 (Tschernobyl). In der markanten Eintragslage aus dem Jabr 1986 ist die Radioaktivität besonders hoch. Dies spricht für einen bohen Anteil an belastetem Bodenmaterial, welches in den See gelangte.

Fig. 2: ${ }^{137} \mathrm{Cs}$ dating (J. -L. Reyss) of the short core Mo_05_P3 with activity peaks indicating nuclear fall-out in 1963 (nuclear weapon tests) and in 1986 (Chernobyl). High peak activity of Chernobyl dues to high amounts of contaminated soil material washed into the lake by a debris flow occurred three month after the reactor accident. 
Jahren 1986 (Reaktorunglück Tschernobyl) und 1963 (Höhepunkt der oberirdischen Atomwaffentests) lassen sich auch in den Ablagerungen des Mondsees wiederfinden und entsprechen den Datierungen durch die Jahreslagenzählung.

\section{Beschreibung der Sedimente}

\section{Jahreslagen (Warven)}

In den Sedimenten zeichnet sich eine deutliche Laminierung durch den Wechsel von hellen und dunklen Lagen ab, wobei ein Lagenpaar einem Jahr entspricht. Eine repräsentative Jahreslage wird hauptsächlich durch eine Frühjahrslage, Sommerlage und Herbstlage aufgebaut. Die Frühjahrslage enthält gut ausgebildete Kalzitkristalle (Korngröße bis $5 \mu \mathrm{m}$ ) und Schalen von abgestorbenen Kieselalgen (Diatomeen). Die markante und hell erscheinende Sommerlage besteht fast ausschließlich aus kleineren Kalzitkristallen (Abb. 3). Die charakteristische weiße Färbung der Sommerlagen wird durch die biochemische Kalzitfällung im Sommer verursacht, wenn durch die Algenblüte dem Wasser $\mathrm{CO}_{2}$ entzogen wird, sich dadurch das Kalk-KohlensäureGleichgewicht verschiebt und Kalzit, welcher gelöst als Kalziumkarbonat im Wasser vorliegt, ausfällt. Die Herbstlage enthält entweder Diatomeen oder eine weitere Lage aus Kalzitkristallen. Die Winterlage ist hingegen nur schwach ausgeprägt. Die
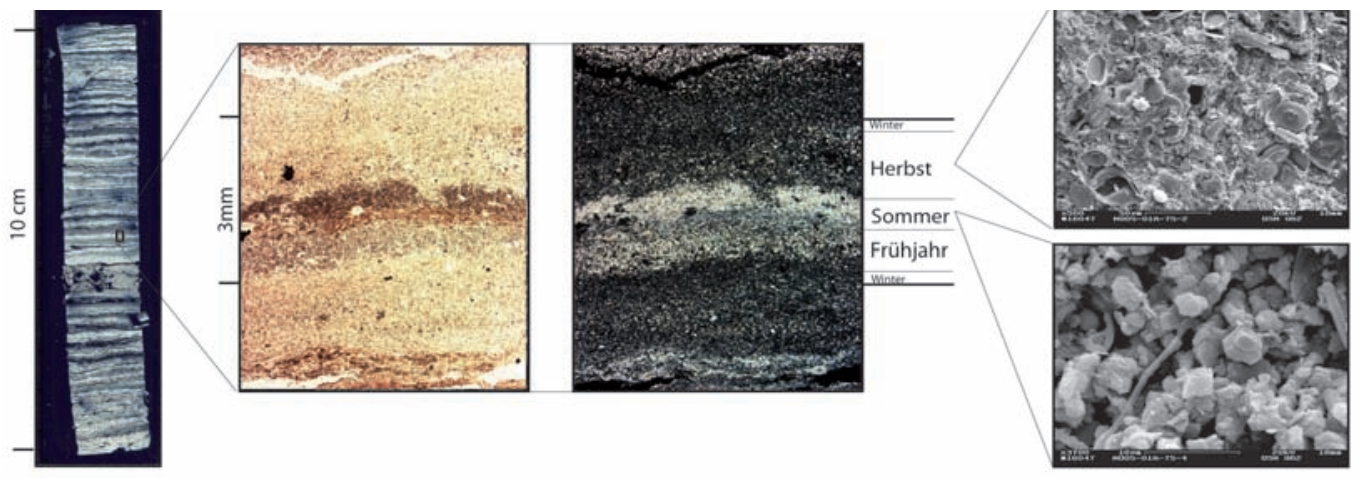

Abb. 3: links: Dünnschlifffoto der obersten zehn Zentimeter des Bobrkerns Mo_05_P3 unter polarisiertem Licht mit den Jahreslagen aus den letzten 30 Jahren; mitte: Jahreslage 1989 unter linear polarisiertem Licht und mit gekreuzten Polarisatoren; rechts: Rasterelektonenmikeroskopaufnahmen von einer sommerlichen Kalzitlage (unten) und einer herbstlichen Lage mit Kieselalgen, organischem Material und Ton (oben).

Fig. 3: left: Thin section image of the top $10 \mathrm{~cm}$ of the short core Mo_05_P3 using polarized light showing the varves of the past 30 years; middle: varve 1989 using linear polarized light and with crossed polarizers; right: SEM images of a summer calcite layer (lower image) and an autumn layer with diatoms, organic matter and clay (upper image). 
niedrige Sedimentationsrate in den Wintermonaten erklärt sich aus der Ablagerung lediglich weniger feiner, meist toniger Schwebpartikel. Im Sommerhalbjahr lassen sich zusätzlich kurzfristige Sedimenteinträge aus dem Einzugsgebiet des Sees durch die Flüsse als deutliche Eintragslagen identifizieren. Diese Eintragslagen bestehen hauptsächlich aus organischem und minerogenem Material (Detritus) und sind in den Dünnschliffen und teilweise auch makroskopisch im Sedimentprofil sichtbar. Sie können anhand ihres Auftretens innerhalb der saisonalen Abfolge entsprechend den Jahreszeiten zugeordnet werden. Im Deltabereich der Fuschler Ache sind die Eintragslagen wie zu erwarten häufiger und deutlich dicker vertreten.

\section{Sedimentationsraten}

An der tiefsten Stelle des südlichen Beckens (Bohrkern Mo_05_P3) beträgt die durchschnittliche Sedimentationsrate ca. 2,5 mm/Jahr, was in den letzten 100 Jahren in der Akkumulation von ca. $25 \mathrm{~cm}$ Sediment resultierte. Im Zeitraum von 1968 bis 1983 kann eine leichte Erhöhung der Sedimentationsrate festgestellt werden. Die menschlich verursachte Eutrophierung durch verstärkte Einleitung ungeklärter Siedlungsabwässer (Jagsch, 1982) führte zu massivem Algenwachstum im Sommer und dementsprechend verstärkter Ablagerungen von Kieselalgenskeletten.

\section{Vergleich der Eintragslagen im Seebecken}

Eintragslagen werden als Ereignislagen oder auch detritische Lagen bezeichnet und können anhand der Zusammensetzung des Eintrags charakterisiert werden. Im Mündungsbereich der Flüsse (proximaler Bereich) sind die Lagen dicker ausgeprägt. Weiter entfernt von der Eintragsquelle (distaler Bereich) wird hingegen deutlich weniger und feineres Material abgelagert. Die Schneeschmelze im Frühjahr führt in der Nähe des Deltas der Fuschler Ache zu einem zusätzlichen Eintrag von klastischem Material, der in den Sedimentprofilen deutlich sichtbar ist, und dort zu einer höheren Sedimentationsrate von bis zu $6 \mathrm{~mm} / \mathrm{J}$ ahr beiträgt. Unabhängig vom Zufluss

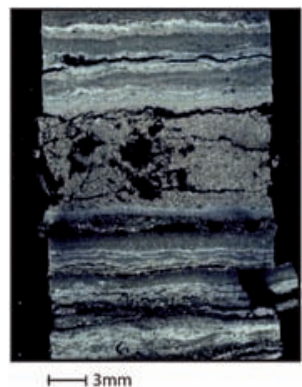

Eintragslage $5-1986$ (makroskopisch)

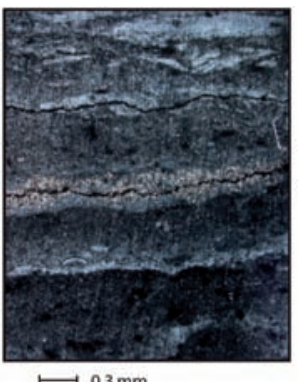

$\longmapsto 0,3 \mathrm{~mm}$

Eintragslage 9- 1936 (mikroskopisch)
Abb. 4: Dünnschlifffotos (gekreurte Polarisatoren) mit Jahreslagen und eingeschalteten Eintragslagen.

Fig. 4: Thin section images (crossed polarizers) with varves and intercalated event layers. 
zeigt der Vergleich der Eintragslagen in den einzelnen Kernprofilen, dass generell zwischen dickeren und schmaleren, oft nur mikroskopisch erkennbaren Eintragslagen (Abb. 4) unterschieden werden kann. Dabei lassen sich die dickeren Lagen mit einer Mächtigkeit von 0,1-10 mm nur im Südbecken lokalisieren, wohingegen dünnere Lagen mit weniger als 0,1 mm Mächtigkeit in allen Kernen nachweisbar sind. Anhand der Dünnschliffe lassen sich in allen Kernen 12 markante Eintragslagen innerhalb des Zeitraums der letzten 100 Jahre feststellen und miteinander korrelieren (Abb. 5).

\section{Vergleich von Sedimentarchiv mit historischen Hochwasserereignissen}

Neben der mikroskopischen Auswertung der Sedimentmikrofazies geben geochemische Analysen (Mikro-Röntgenfluoreszens-Analyse) zusätzliche Informationen zum Charakter der Eintragslagen. So können erhöhte Gehalte bestimmter Elemente wie z.B. Magnesium (Abb. 6), welches in Dolomit vorkommt und damit vor allem das Einzugsgebiet des Südbeckens charakterisiert, festgestellt werden. Erhöhte Magnesium-Gehalte reflektieren deshalb auch deutlich die 12 datierten Eintragslagen, die mit historischen Hochwässern und Murabgängen in Zusammenhang gebracht werden können.

Die Datenlage zu den historischen Hochwasserereignissen am Mondsee ist umfangreich. Seit 1895 werden die Wasserstände des Sees täglich aufgezeichnet, so dass bedeutende Hochwasserereignisse gut dokumentiert sind (Abb. 7). Für den Zeitraum ab 1976 standen zusätzlich tägliche Abflussmessungen der Flüsse zur Verfügung. Aus historischen Aufzeichnungen können ebenfalls nützliche Informationen über bedeutende Hochwasserereignisse und Murschäden in den letzten 100 Jahren gewonnenen werden.

Der Vergleich der verfügbaren Daten mit den Eintragslagen zeigt, dass vor allem die Frühjahrs- und Sommerhochwasser in den Sedimenten aufgezeichnet werden. Darunter lassen die großen Sommerhochwasser der Jahre 1954 und 1959 deutliche Sedimenteinträge erkennen. Für das Winterhochwasser 1974 ist hingegen kein signifikanter Sedimenteintrag erkennbar, vermutlich weil dieses Hochwasser durch einen Eisstau auf dem Mondsee ausgelöst wurde. Das prominente Sommerhochwasser 2002 ist wiederum deutlich im Sediment erkennbar, auch wenn es sich durch einen vergleichsweise eher geringen Eintrag von detritischem Material auszeichnet. $\mathrm{Zu}$ den stärksten Hochwasserereignissen zählen die großen Septemberhochwasser in den Jahren 1899 und 1920, welche jedoch kaum durch eine signifikante Erhöhung des detritischen Eintrags belegt sind. Die unterschiedliche Überlieferung von Hochwasserereignissen in den Sedimenten deutet letztlich darauf hin, dass extreme Winter- und Herbsthochwasser weniger Eintragsmaterial in den See führen, 
Klimawandel in Österreich
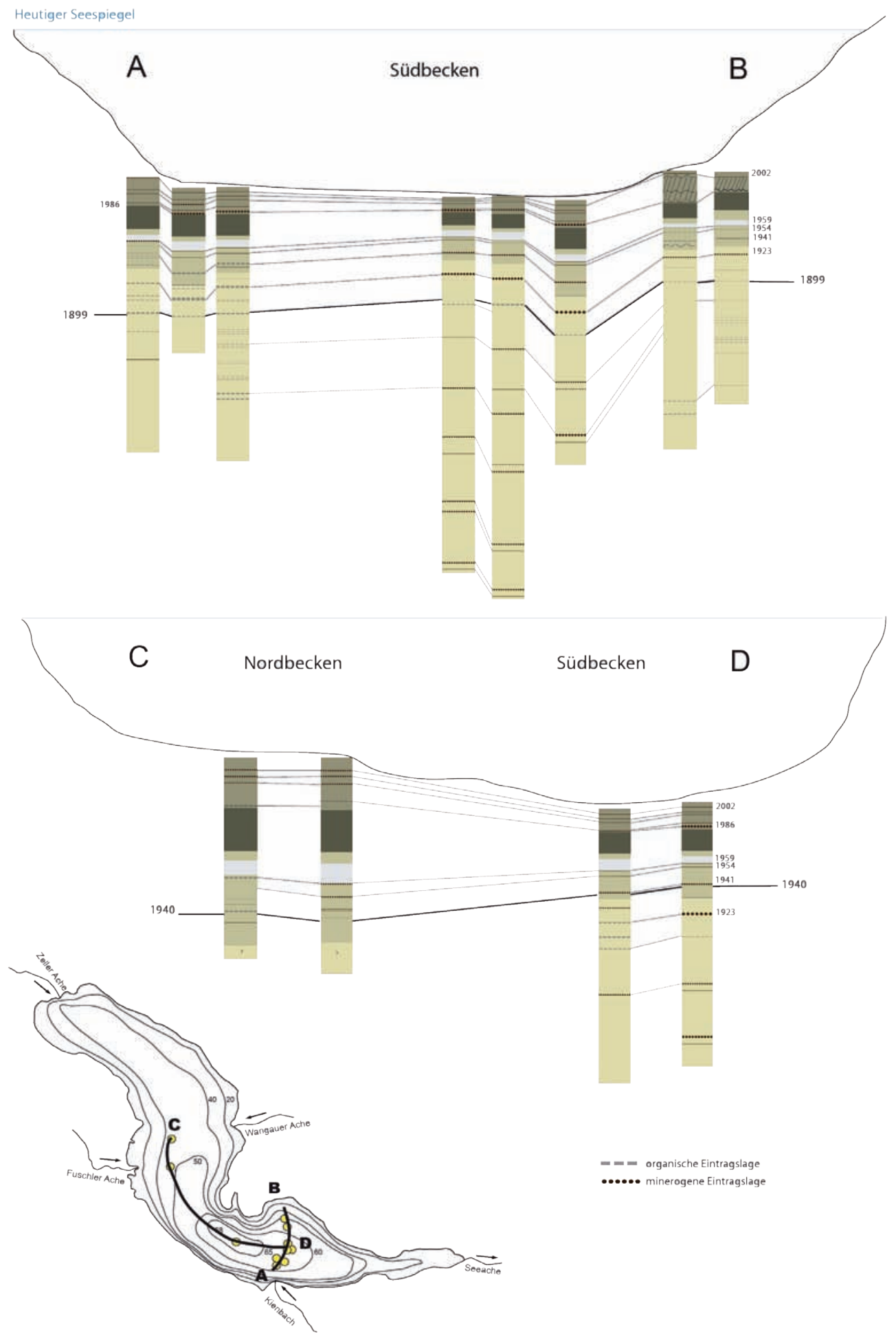
Abb. 5: Korrelation der Ereignislagen entlang zweier Transekte mit den markanten Eintragsereignissen 2002, 1986, 1959, 1954, 1941, 1923 (graue Färbung im Profil korreliert mit der Intensität der Algenblüte und der Kalqitfällung im Sommer, weiße Schicht entspricht feinklastischem Eintrag); a) Transekt A-B (ca. $1 \mathrm{~km})$ : Südbecken mit unterschiedlicher Mächtigkeit und Aufbau besonders dicker Ereignislagen; b) Transekt C-D (ca. 3,5 km): Nord-Südbecken mit erhöhter Sedimentationsrate im Deltabereich der Fuschler Ache

Fig. 5: Correlation between the different event layers along two transects with the pronounced event layers of 2002, 1986, 1959, 1954, 1941, 1923 (grey units in the profile correlate with intensity of algal bloom and precipitated calcite in the summer period, white unit represents fine clastic material) a) transect A-B (ca. $1 \mathrm{~km})$ : southern basin. Especially the thickest detrital layers vary in thickness and composition b) transect C-D (ca. $3.5 \mathrm{~km}$ ): north to south basin with enhanced sedimentation close to the estuary of Fuschler Ache..

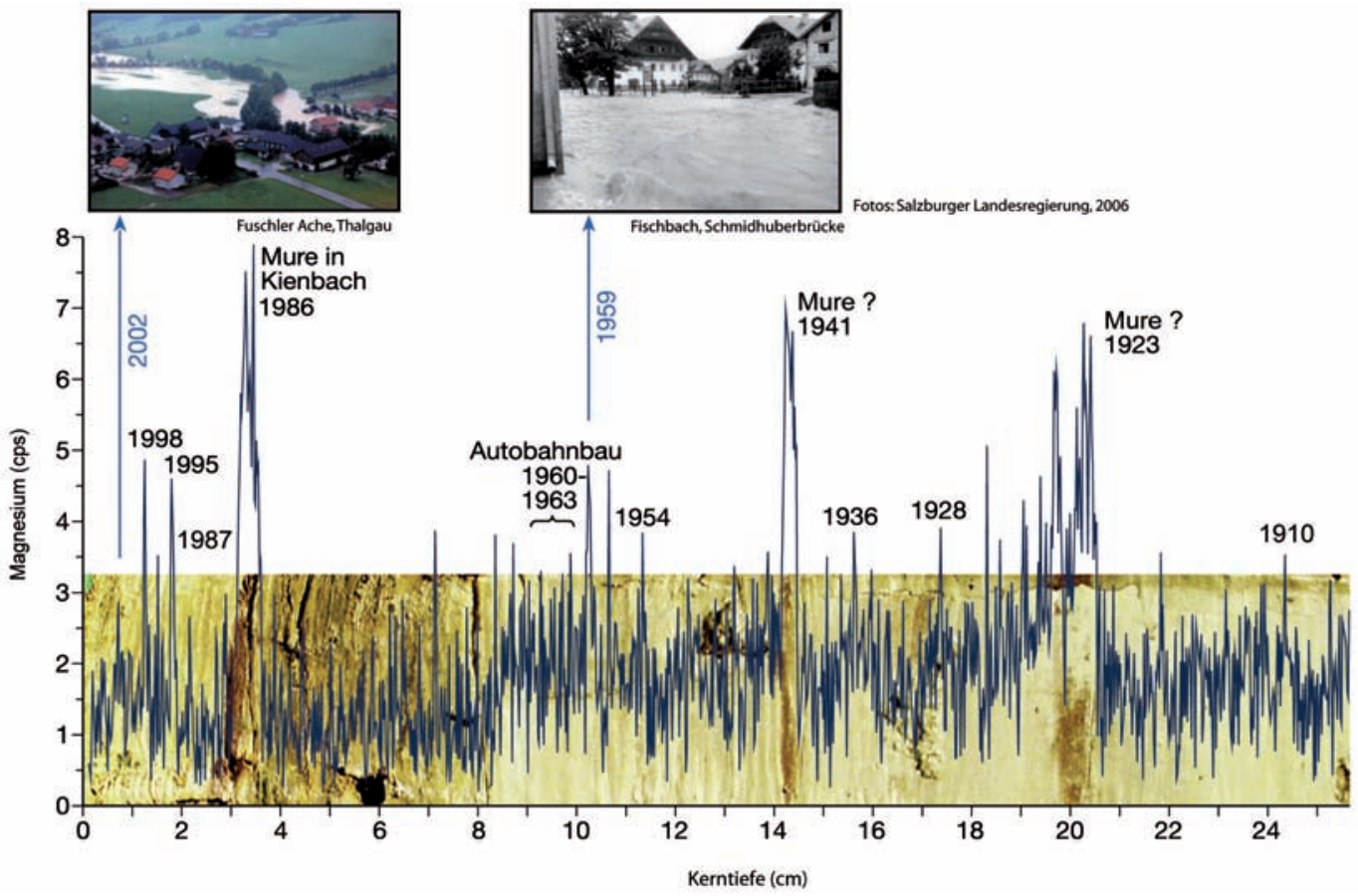

Abb. 6: Mit modernen Mikro-Röntgenfluoreszensmethoden gemessene erböhte Magnesiumgehalte sind Anzeiger für Sedimenteintrag aus dem dolomitischen Einzugsgebiet im Süden des Mondsees, bier am Kur₹kern Mo_05_P3 im Südbecken gemessen. Markiert sind die Eintragslagen, welche mit extremen Hochwasserereignissen im Zeitraum 1905-2005 korrespondieren. Neben den scharf abgegrenzten Ereignislagen fällt besonders auch der Bereich der frühen 60er Jabre durch einen diffus erböbten Magnesium-Gehalt auf. Hier spiegelt sich der Bau der Autobahn in den Jahren 1960-63 wider. Nach Schätzungen erfolgte während dieser Zeit eine Zufuhr von 0,8-1 Mio. $m^{3}$ tonig-lehmigen Bergabraums (Wetzel, 1988), der sich als feinste Partikel im Seebecken verteilte. Für diese Jahre können daher auch entsprechend dickere Jahreslagen nachgewiesen werden.

Fig.6: Modern X-ray fluorecence spectroscopy demonstrates increased magnesium concentrations in the southern basin of Lake Mondsee originating from dolomitic sediments. Pronounced event layers are caused by extreme runoff events as floods and debris flow events between 1905-2005. Besides distinctive event layers corresponding to single events the early 60ies exhibit high Magnesium levels. At this time the nearby highway was constructed. The amount of silt and clay transported by these activities into the lake is estimated to $0.8-1$ mio. $\mathrm{m}^{3}$. Accordingly, for this period thick varves are recorded. 


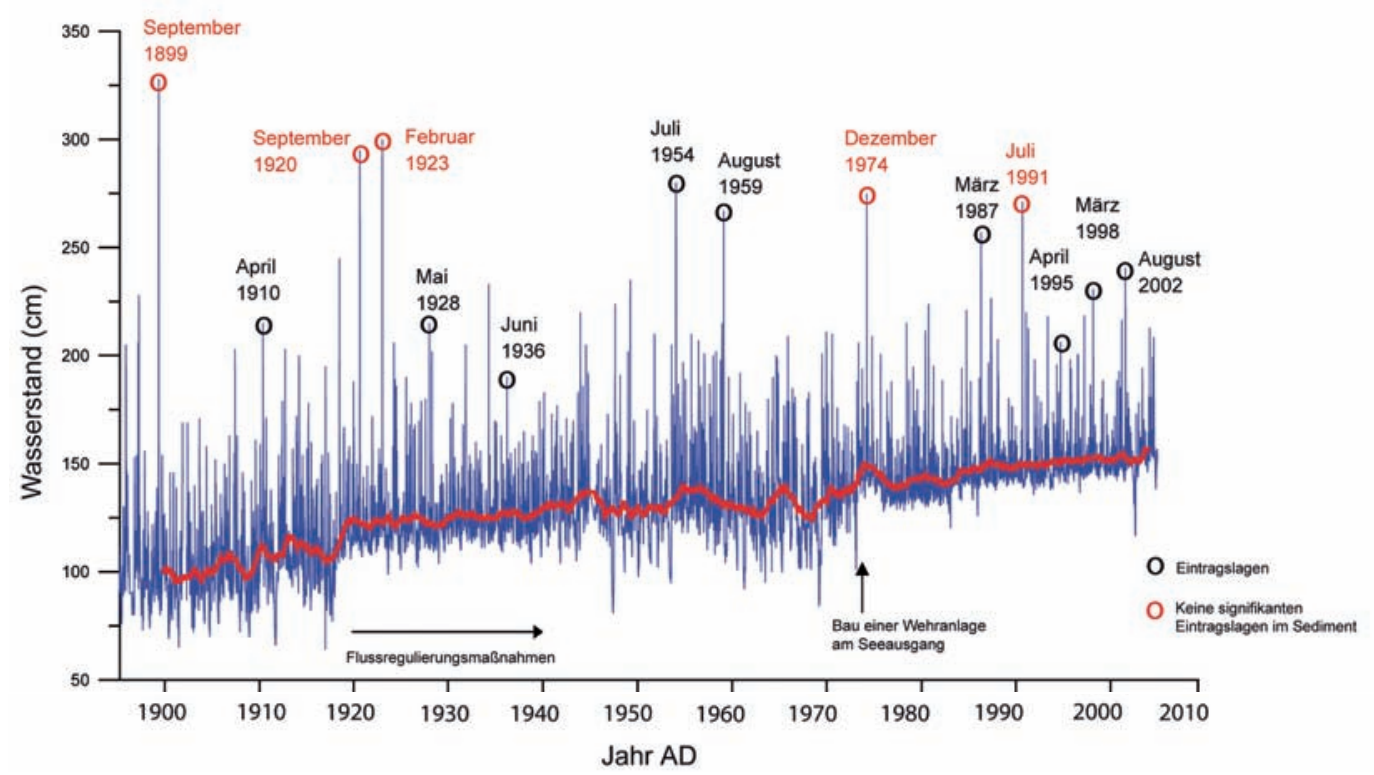

Abb. 7: Tägliche Wasserstände des Mondsees mit zweijährigem Mittel (rot). Schwarz markiert sind die Eintragslagen, die mit Hochwasserereignissen in Zusammenhang stehen und rot markiert sind extreme Hochwasserereignisse obne signifikanten Sedimenteintrag. Der allgemeine Anstieg des Wasserstands des Mondsees wird durch die Flusslaufregulierung der größseren Zufliusse, v. a. der Fuschler Ache in den Jabren 1920-1965 und durch den Bau einer Wehranlage (Klauswehr) am Seeabfluss (Seeache) im Jahre 1972 verursacht. Der Hochwasserstand im Sommer 1991 erfolgte aufgrund einer technischen Störung am Wehr.

Fig. 7: Daily water level of Lake Mondsee and the biannual mean (red). Black symbols indicate flood event layers, red symbols indicate flood events with no significant event layer. The general increase in the water level is attributed to the regulation of inflowing rivers during 1920-1965 and the construction of the dam at the outlet in 1972. The high water level of 1991 was caused by technical problems of the dam.

Frühjahrs- und Sommerhochwasser sich dagegen durch höheren Sedimenteintrag auszeichnen. Mögliche Gründe dafür sind in der jahreszeitlich unterschiedlichen Temperaturschichtung des Sees zu suchen. Außerdem spielt die Stärke des Sedimentabtrags während Starkregenereignissen eine wichtige Rolle, die wiederum von der Intensität des Niederschlags, von dem Durchfeuchtungsgrad des Bodens vor dem Niederschlagsereignis und außerdem sicherlich von der jahreszeitlich vorherrschenden Großwetterlage abhängt.

Besondere Erwähnung finden in der Ortschronik auch katastrophale Murereignisse, welche sich wiederholt an den steilen Hängen am Südufer des Sees, am Rand der nördlichen Kalkalpen, ereignen. Beim letzten bekannten derartigen Ereignis wurde am 18. Juli 1986 ein lokaler Gewitterregen an der Station Scharfling mit 42 mm Niederschlag registriert. Dieser löste in diesem ansonsten sehr trockenen Jahr einen lokalen Erdrutsch im Gebiet des Kienbachs aus. In einer Schlammlawine wurde die 
Pflanzen- und Bodendecke mitgerissen und in den See transportiert, wo sie heute eine mehrere Millimeter bis zu Zentimeter dicke Lage aus Pflanzenresten und klastischem Sediment bildet. Diese Lage ist durch deutlich erhöhte ${ }^{137} \mathrm{Cs}$-Werte charakterisiert (Abb. 2), was dadurch zu erklären ist, dass dieses Murereignis nur knapp 3 Monate nach dem Reaktorunfall von Tschernobyl stattfand, und der abgetragene Boden zu dieser Zeit bereits stark mit ${ }^{137} \mathrm{Cs}$ belastet war. Ähnlich mächtige Lagen mit vergleichbarer Zusammensetzung, wie die erhöhten Gehalte an Magnesium zeigen, finden sich auch in den Jahreslagen von 1923 und 1941 wieder. Deshalb werden in diesen Jahren ebenfalls lokale Erdrutsche mit bedeutendem Sedimenteintrag aus dem Einzugsgebiet in den See vermutet (Abb. 6). Ein historischer Beleg dafür steht allerdings noch aus.

\section{Schlussfolgerungen}

Die neu gewonnenen Informationen über Eintragslagen in Seesedimenten des Mondsees verbessern das Verständnis von extremen Eintragsereignissen wie Hochwasserkatastrophen und Murenabgängen. Der Vergleich der Seesedimente mit den historischen Daten aus den letzten 100 Jahren (Tab. 1) konnte zeigen, dass das Archiv der Seesedimente ein hohes Potenzial für die Rekonstruktion von extremen Niederschlagsereignissen auch für prä-instrumentelle Zeit besitzt. Mit diesen Erkenntnissen ist die Möglichkeit gegeben, einen Hochwasser- und Murkalender zu erstellen und klimatische Fragestellungen, insbesondere bezüglich der Wiederkehr extremer Niederschlagsereignisse, zu untersuchen.

Tab. 1 : Übersicht zu den im Sediment seit 1899 aufgezeichneten Eintragsereignissen (Muren und Hochwasser)

\begin{tabular}{clll}
\hline Eintragslage & Jahr & Mächtigkeit & Ereignis \\
\hline 1 & 2002 & Mikroskopisch & Hochwasser \\
2 & 1998 & Mikroskopisch & Hochwasser \\
3 & 1995 & Mikroskopisch & Hochwasser \\
4 & 1987 & Mikroskopisch & Hochwasser \\
5 & 1986 & Makroskopisch & Mure \\
6 & 1959 & Mikroskopisch & Hochwasser \\
7 & 1954 & Mikroskopisch & Hochwasser \\
8 & 1941 & Makroskopisch & Mure? \\
9 & 1936 & Mikroskopisch & Hochwasser \\
10 & 1928 & Mikroskopisch & Hochwasser \\
11 & 1923 & Makroskopisch & Mure? \\
12 & 1910 & Mikroskopisch & Hochwasser \\
\hline
\end{tabular}




\section{Klimawandel in Österreich}

\section{Dank}

Wir danken dem Limnologischen Institut Mondsee, und dort vor allem D. Danielopol, R. Schmidt, J. Knoll und K. Maier für die Unterstützung bei der Entnahme der Kurzkerne und die Bereitstellung der Wasserstandsdaten des Mondsees. Diese Studie entstand im Rahmen einer Diplomarbeit und im Zusammenhang mit dem im Rahmen des ESF EuroCLIMATE Programms von der DFG geförderten Projekt „DecLakes“ (BR 2208/2-2). Allen Projektpartnern danken wir weiterhin für viele nützliche und anregende Diskussionen. Für die Ausführung der ${ }^{137}$ Cs-Datierung danken wir J.-L. Reyss (LSCE, Gif sur Yvette). Außerdem möchten wir uns für die freundlichen Auskünfte bei Herrn A. Jagsch (Mondsee), den Mitarbeitern des Pfahlbaumuseums Mondsee und der Familie Hemetsberger (Mondsee) bei der Recherche historischer Ereignisse bedanken.

\section{Literaturverzeichnis}

Brauer, A. 2004: Annually laminated lake sediments and their palaeoclimatic relevance. In: Fischer, H., Kumke, T., Lohmann, G., Flöser, G., Miller, H., von Storch, H., Negendank, J. F. W., (Eds.), The climate in historical times. Towards a synthesis of Holocene proxy data and climate models. 109-127. Springer, Berlin / Heidelberg.

Dokulil, M. \& Skolaut, C. 1986. Succession of phytoplankton in a deep stratifying lake: Mondsee, Austria. Hydrobiologia 138: 9-24.

Dokulil, M.T., Jagsch, A., George, G.D., Anneville, A., Jankowski, Th., Wahl, B., Lenhart, B., Blenckner, T. \& Teubner, K. 2006. Twenty years of spatially coherent deep-water warming in lakes across Europe related to the North Atlantic Oscillation. - Limnol. Oceanogr. 51: 2787-2793.

Helbig, J. 1987: Sedimentologische Kartierung im Mondsee und Untersuchungen zur Phosphor- Rücklösung an Sedimenten des Mondsees und des Obertrumer Sees (Österreich). Diplomarbeit (Institut für Geologie und Dynamik der Lithosphäre der Georg-August-Universität Göttingen)

Herrmann, M. 1990: Oberflächenkartierung der Mooswinklbucht/Mondsee und Untersuchungen zur Tiefenstruktur. Diplomarbeit (Institut für Geologie und Dynamik der Lithosphäre der Georg-August-Universität Göttingen)

Horsthemke, E. 1986: Sedimentologische Untersuchungen zum Problem von Seespiegelschwankungen im Bereich der neolithischen Siedlungen der Station See im Mondsee (Salzkammergut, Österreich). Diplomarbeit (Institut für Geologie und Dynamik der Lithosphäre der Georg-August-Universität Göttingen)

Jagsch, A., Megay, K. 1982: Seereinhaltung in Österreich. Wasserwirtschaft 6: 155-163

Klee, R. \& Schmidt, R. 1987. Eutrophication of Mondsee (Upper Austria) as indicated by the diatom stratigraphy of a sediment core. Diatom Research 2: 55-76.

Merkt, J. 1971: Zuverlässige Auszählung von Jahresschichten in Seesedimenten mit Hilfe von GroßDünnschliffen. Archiv für Hydrobiologie 69: 145-154.

Mudelsee, M. 2003: No upward trends in the occurrence of extreme floods in central Europe. Nature 425: 166-169.

Schmidt, R., Müller, J., Froh, J. 1985: Laminated sediments as a record of increasing eutrophication of Lake Mondsee. Contributions to the paleolimnology of Trumer lakes (Salzburg and the Lake Mondsee, Attersee and Traunsee (Upper Austria). Limnologisches Institut Mondsee.

Wetzel, B. 1988: Sedimentologische Kartierung im Mondsee und ein Versuch zur Datierung der Sedimente anhand von Phosphorgehalten unter Berücksichtigung der Sanierungsmaßnahmen am Mondsee (Österreich). Diplomarbeit (Institut für Geologie und Dynamike der Lithosphäre der Georg- August- Universität Göttingen) 


\title{
Paläoklimainformationen aus Kenngrößen der Gletscher- massenbilanz - Beispiele für die Alpen seit der ausgehenden Kleinen Eiszeit
}

\author{
Wolfgang Schöner
}

Zentralanstalt für Meteorologie und Geodynamik, Wien

\section{Abstract}

Paleoclimate information from measures of glacier mass-balance-examples from the Alps in the late Little Ice Age period

Glaciers are important proxy data sources in paleoclimatology. The mass balance is the undelayed climate signal of a glacier. This paper deals with examples of proxy climate information derived from glacier mass balance measurements as well as from indirect measures of mass balance (volume change; length change; equilibrium line altitude, ELA; accumulation area ratio, AAR) for the late little ice age period. All these data sources provide multiple climate proxy-information which can not be extracted easily into the single components, e.g. air temperature and precipitation. Temporal high resolution and long term series of glacier changes are only available from front position measurments. Driving powerful glacier models with series of glacier lengths enables to compute air temperatures and precipitation back to the $18^{\text {th }}$ century. All other mass balance measures (volume changes, mass balances, ELA, AAR) either have a much lower temporal resolution or a much shorter series length and thus are less powerful for paleoclimatology.

\section{Gletscher als Klimainformation}

Gletscher sind seit langem eine wichtige Datenquelle in der Paläoklimaforschung. Zwei unterschiedliche Konzepte der Proxydatengenerierung aus Gletschern können unterschieden werden:

a) Analyse der im Eisgefüge gespeicherten Klimainformation (Analyse von Eisbohrkernen) wobei sowohl das Wasser des Eises als auch die eingeschlossene Luft untersucht werden können. Die Literatur darüber ist sehr umfangreich. In den Alpen existieren derartige Untersuchungen nur für den wesentlich höheren Bereich der Westalpen (z.B. Wagenbach et al., 1998). Die Gletscher der Ostalpen sind auf Grund der geringeren Seehöhen und der damit verbundenen sommerlichen Schmelze nicht in der Lage, entsprechende Klimainformation im Eis oder Firn zu speichern. Da derartige Proxydaten für die österreichischen Alpen nicht vorliegen und auch den Rahmen dieser Arbeit sprengen würden, werden sie in diesem Artikel nicht weiter behandelt. 
b) Proxydaten die aus der Untersuchung der Gletschermassenbilanz und damit in Zusammenhang stehenden Größen (Volumsänderungen, Längenänderungen, Höhe der Gleichgewichtslinie, Flächenverhältnis Akkumulationsgebiet zu Gesamtfläche) abgeleitet werden. Im weiteren Sinne beziehen sich auch Klimarekonstruktionen aus Moränenständen, Expositionsdatierungen, Flechtenmessungen und ähnliches auf die Massenbilanz eines Gletschers. Derartige Proxydaten liegen für die österreichischen Gletscher vor und werden daher im Folgenden teilweise näher beschrieben.

\section{Zusammenhang Klima - Gletscher}

Der generelle Zusammenhang zwischen dem Klima und dem Gletscherverhalten wird heute recht gut verstanden. Die wichtigste Größe zur Beschreibung des Zusammenhanges ist die Massenbilanz des Gletschers B, die sich aus der Akkumulation C und der Ablation A zusammensetzt:

$$
\mathrm{B}=\mathrm{C}+\mathrm{A}(\mathrm{kg})
$$

Das Intervall, über das die Größe B bestimmt wird, ist angepasst an den natürlichen Zyklus der Jahreszeiten, 1 Jahr und beginnt mit guter Näherung mit dem 1. Oktober und endet mit dem 30. September des Folgejahres. Um eine Vergleichbarkeit verschiedener Gletscher zu ermöglichen, werden die Bilanzterme durch die Gletscherfläche dividiert und so die spezifischen Bilanzgrößen errechnet:

$$
\mathrm{b}=\mathrm{c}+\mathrm{a}\left(\mathrm{kg} / \mathrm{m}^{2}\right)
$$

Die Aufteilung in die zwei Terme Akkumulation und Ablation legt bereits nahe, dass es sich bei den aus Gletschermassenbilanzen abgeleiteten Klimainformationen um kombinierte Klimaparameter handelt (Abbildung 1), wobei auch Akkumulation

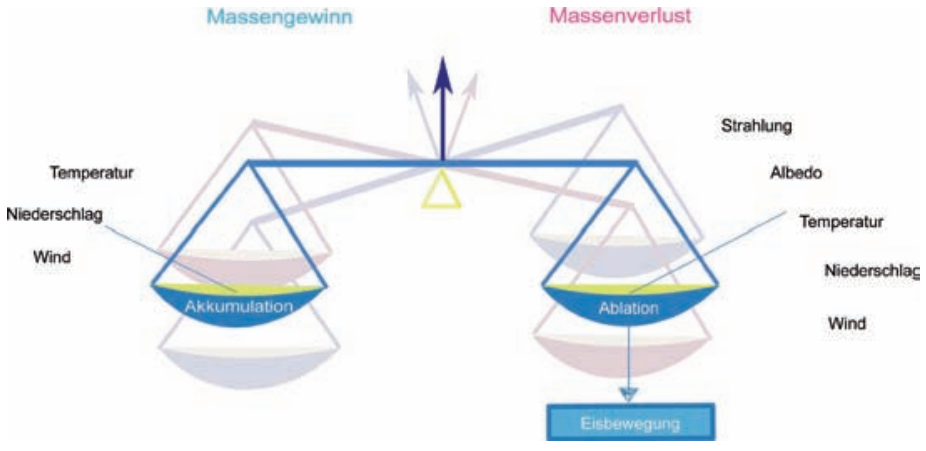

Abb. 1: Klimatische Ursachen der Massenbilanz-Komponenten Akkumulation und Ablation

Fig.1: Climatic causes for accumulation and ablation in glacial mass balances 
und Ablation selbst von verschiedenen Klimakenngrößen bestimmt werden. Zur Massenbilanzgröße Akkumulation tragen vor allem folgende Klimaparameter bei:

- Niederschlag

- Windgeschwindigkeit und Windrichtung

Die kombinierte Gletschergröße Ablation wird insbesondere durch folgende Klimaparameter bestimmt:

- Strahlungsbilanz

- $\quad$ Lufttemperatur

- Windgeschwindigkeit und Windrichtung

Betrachtet man das doch sehr komplexe Wirkungsgefüge der klimatologischen Einflussgrößen (Abbildung 1), dann mag es verwundern, dass man aus Gletschern überhaupt Klimainformationen ableiten kann. Sowohl Akkumulation als auch Ablation können jedoch durch Vereinfachungen gut angenähert werden:

$$
\mathrm{c} \approx \mathrm{b}_{\mathrm{w}} \approx \text { f. } \mathrm{P}_{\mathrm{w}}
$$

$\left(\mathrm{b}_{\mathrm{w}}\right.$ ist die spezifische Wintermassenbilanz und $\mathrm{P}_{\mathrm{w}}$ der Winterniederschlag in $\mathrm{mm}, \mathrm{f}$ ist ein Proportinalitätsfaktor)

Diese Vereinfachung sagt aus, dass die Akkumulation vorwiegend im Winter stattfindet und im Wesentlichen von der entsprechenden Niederschlagsmenge für diese Periode bestimmt wird. In Abbildung 2 sind Messungen der Wintermassenbilanz den entsprechenden Winterniederschlägen für österreichische Gletscher, von denen Messungen vorliegen, gegenübergestellt. Es zeigt sich, dass der Faktor $\mathrm{f}$ zwischen ca. 1,0 und 4,5 schwankt und dass für niederschlagsreichere Regionen die Schwankungsbreite kleiner wird.

Abb. 2: Beziehung zwischen Winterniederschlag und dem Verbältnis Wintermassenbilanz zu Winterniederschlag (aus Schöner und Böhm, 2007)

Fig. 2: Relationship between winter precipitation and the ratio of winter mass balance and winter precipitation (from Schöner and Böhm, 2007)

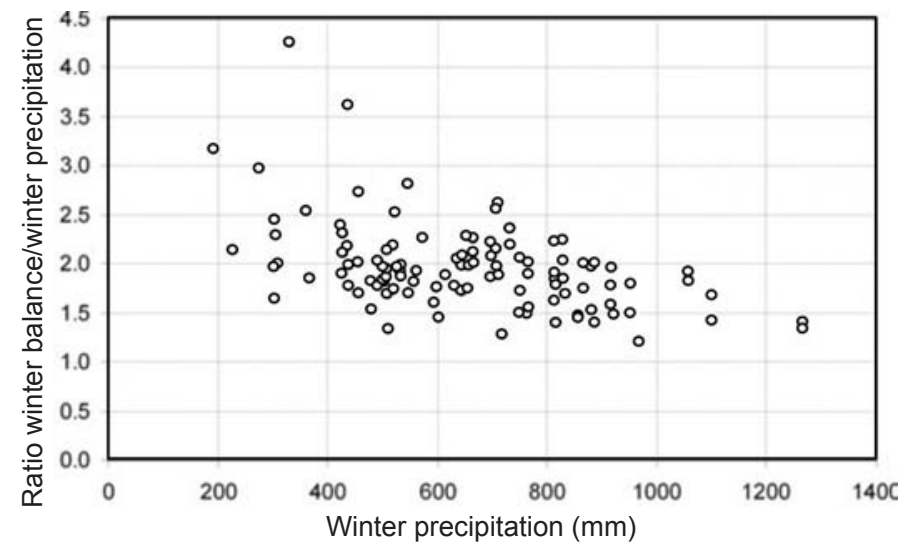


Die Ablation an Alpengletschern wird vorwiegend durch die Energiebilanz im Sommer bestimmt (Hermann und Kuhn, 1990). Ein Vergleich von Messungen der Energiebilanz an verschiedenen Gletschern der Alpen zeigt, dass die kurzwellige Strahlungsbilanz die wesentliche Einflussgröße ist (z.B. Kuhn, 2004). In weiterer Folge haben mehrere Arbeiten (z.B. Ohmura, 2001) nachgewiesen, dass die Lufttemperatur, insbesondere über die so genannte positive Temperatursumme, sehr gut die Energiebilanz über Schnee- oder Eisflächen beschreibt (wobei die Annahme einer Oberflächentemperatur von $0{ }^{\circ} \mathrm{C}$ erfüllt sein muss). Daher ist folgende Vereinfachung möglich:

$$
\mathrm{a} \approx \mathrm{b}_{\mathrm{s}} \approx \mathrm{g} \cdot \mathrm{T}_{\mathrm{s}}
$$

Das bedeutet, dass die Ablation recht gut mit der Sommertemperatur Ts angenähert werden kann. Die Massenbilanz b wird also durch die zwei Größen Winterniederschlag $\mathrm{P}_{\mathrm{w}}$ und Sommertemperatur $\mathrm{T}_{\mathrm{s}}$ sehr gut beschrieben. Es ergibt sich somit:

$$
\mathrm{b}=\mathrm{f} . \mathrm{P}_{\mathrm{w}}+\mathrm{g} \cdot \mathrm{T}_{\mathrm{s}}+\mathrm{h}
$$

Dabei ist h eine Konstante, die für jeden Gletscher unterschiedlich ist und die die topographischen Gegebenheiten eines Gletschers (Expostion, Seehöhe, etc.) beschreibt. Dieser Zusammenhang gilt unter stationären Bedingungen. Setzt man anstatt der Lufttemperatur die positive Temperatursumme in obige Formel ein, so handelt es sich bei der Größe g um den Gradtagfaktor, der meist Werte zwischen -4 bis $-8 \mathrm{~mm} /{ }^{\circ} \mathrm{C}$ Tag annimmt (Kuhn, 2004).

Für die Alpengletscher kann mit gutem Erfolg dieser Zusammenhang nochmals vereinfacht werden, indem man

$$
\mathrm{b} \approx \mathrm{f}(\mathrm{a}) \approx \mathrm{k} \cdot \mathrm{T}_{\mathrm{s}}+\mathrm{h} \text { setzt. }
$$

Diese Vereinfachung ist im ersten Moment etwas überraschend, da man intuitiv annehmen würde, dass die gefallene Schneemenge im Winter die Bilanz über das gesamte Jahr (mit)bestimmen sollte. Abbildung 3 zeigt am Beispiel des Wurtenkeeses und des Goldbergkeeses (Hohe Tauern), warum diese Vereinfachung jedoch möglich ist. Die zeitliche Variabilität der Wintermassenbilanz ist nämlich wesentlich kleiner als die Variabilität der Sommermassenbilanz, die die gleiche Größenordnung besitzt wie die Variabilität der Jahresbilanz. Mehrere Untersuchungen (z.B. Hoinkes und Steinacker, 1975, Hammer, 1993) haben für die Alpengletscher für die Periode der Massenbilanzmessungen die Gültigkeit dieser Vereinfachung recht gut bestätigt. Oerlemans (2005) hat diese Vereinfachung (Abhängigkeit der Massenbilanz nur von Sommertemperatur) mit Erfolg für die Ableitung von jährlichen Temperaturwerten aus Messwerten von Gletscherlängenänderungen angewandt. 

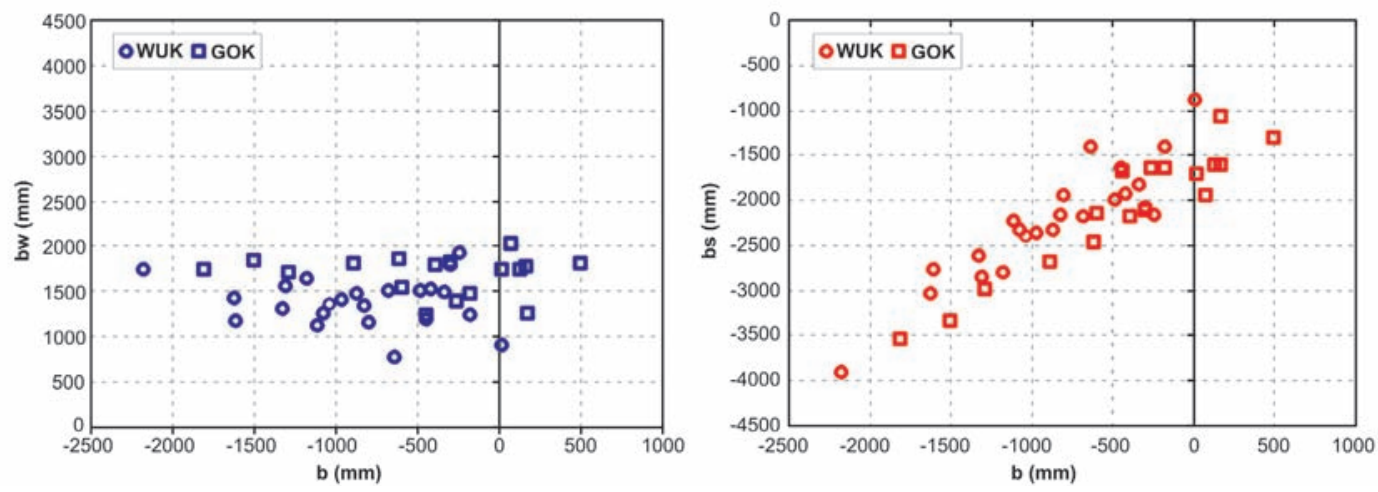

Abb. 3: Beziehung zwischen Winterbilanz bw und Jahresbilanz b (links) und Sommerbilanz bs und b (rechts) für Goldbergkees und Wurtenkees in den Hohen Tauern.

Fig. 3: Relationship between winter balance bw and annual balance b (left) and summer balance bs and b (right) for Goldbergkees and Wurtenkees in Hohen Tauern.

Die Stationarität der Faktoren $\mathrm{f}$ und $\mathrm{g}$ ist eine wesentliche Annahme für die Ableitung von Temperatur- beziehungsweise Niederschlagswerten - konnte jedoch bis jetzt nicht wirklich überprüft werden. Die genauere Analyse des Zusammenhanges zwischen Gletschermassenbilanz und Klimavariablen für verschiedene Regionen weltweit, legt nahe, dass $\mathrm{f}$ und $\mathrm{g}$ von der thermischen und hygrischen Kontinentalität der jeweiligen Region bestimmt werden. Ähnlich wie eine räumliche Änderung sollte daher auch eine zeitliche Änderung der Kontinentalität des Klimas die Faktoren $\mathrm{f}$ und $\mathrm{g}$ ändern (siehe z.B. Schöner et al., 2000). Eine Stationarität von $\mathrm{f}$ und $\mathrm{g}$ ist daher nicht wahrscheinlich.

Zusätzlich gibt es in der Wirkungskette Klima-Gletscherverhalten auch mehrere Rückkoppelungen, die die modellhafte Abbildung des Gletscherverhaltens aus Klimadaten insbesondere für lange Zeitintervalle schwierig machen. Zu den wichtigsten Rückkoppelungen zählt die der Albedo, insbesondere für die Schneedecke, wobei es sich um eine positive (verstärkende) Wirkung handelt. Eine Verminderung der Albedo führt zu einer verstärkten Energieaufnahme aus der kurzwelligen Strahlung und dadurch zu einer verstärkten Schmelze. Die verstärkte Schmelze erhöht jedoch den Flüssigwassergehalt und setzt dadurch wieder die Albedo weiter herab und erzeugt so den verstärkenden Kreislauf.

Schöner und Böhm (2007) haben ein statistisches Massenbilanzmodell für die Alpengletscher entwickelt, das neben klimatischen Prädiktoren auch topographische Prädiktoren berücksichtigt. Ein derartiger Ansatz ist sinnvoll, da die Massenbilanz 
eines Gletschers sehr stark von der Seehöhe der Gletscherzunge oder der Exposition des Gletschers bestimmt wird. Zusätzlich wurden in diesem Modell auch zeitliche Änderungen der Gletschertopographie berücksichtigt, sowie nichtlineare Zusammenhänge (z.B. zwischen dem Anteil des festen Niederschlags und der Lufttemperatur) abgebildet. Das Modell zeigt für die Kalibrierungsperiode eine sehr gute Performance, ist aber nicht in der Lage, den starken Massenverlust vieler Alpengletscher nach 1850 richtig wiederzugeben (siehe Schöner und Böhm, 2007). Dieses Resultat deckt sich mit einem wesentlichen Ergebnis aus der Arbeit von Oerlemans (2005), dessen rekonstruierte Temperaturen für den Alpenraum deutlich niedriger sind als die Messungen aus HISTALP (Auer et al., 2007). Die Modellierungen von Schöner und Böhm (2007) erfolgten jedoch noch ohne der „Early Instrumental Correction“ der HISTALP Lufttemperatur (Böhm, dieser Band).

\section{Historische Dokumente}

Darstellungen der Gletscher in Gemälden datieren bis ins 16. und 17. Jahrhundert zurück und sind daher interessante Dokumente über Gletscher für die ausgehende Kleine Eiszeit. Während die Gletscher in den frühen Werken noch verzerrt dargestellt wurden, existieren aus dem 18. Jahrhundert schon sehr wirklichkeitsnahe Darstellungen, die für semiquantitative Aussagen genutzt werden können. Herausragende Beispiele sind die Gemälde von F. Simony, E. Compton, oder T. Ender.

Als Beispiel ist in der Abbildung 4 ein Gemälde von Thomas Ender zu sehen, welches das Goldbergkees in den Hohen Tauern im Jahr 1834 zeigt. Auch wenn einige Details in dem Gemälde übertrieben dargestellt sind, ist eine sehr gute Abschätzung der Eisausdehnung möglich. Die Ausdehnung entspricht etwa der maximalen Eisausdehnung zum Ende der Kleinen Eiszeit.

Neben diesen bildhaften Darstellungen der Gletscher existieren auch wichtige Informationen über Gletscheränderungen aus historischen Quellen, zum Beispiel des Bergbaues. Dieser befand sich sehr oft in den Höhenlagen der Gletscher, sodass Gletscherzuwachs, Gletscherschwund oder die Schneebedingungen einen Einfluss auf die Wirtschaftlichkeit der Betriebe hatten. Einen Einblick in die enge Verflechtung des Bergbaues mit den Gletschern am Beispiel des Goldbergbaues im Sonnblickgebiet (Hohe Tauern) gibt die Posepny-Karte aus dem Jahr 1879 (Abbildung 5). 
Abb. 4: Das Goldbergkees in den Hohen Tauern im Jahr 1834 (Ölgemälde von Thomas Ender, Liechtensteinmuseum Wien)

Fig. 4: The Goldbergkees in the Hohen Tauern in 1834 (Oil picture by Thomas Ender, Liechtensteinmuseum Vienna). Although some details are exaggerated the picture allows for a reliable assessment of the extent of the ice which corresponds approximately to its maximal size at the end of the Little Ice Age.

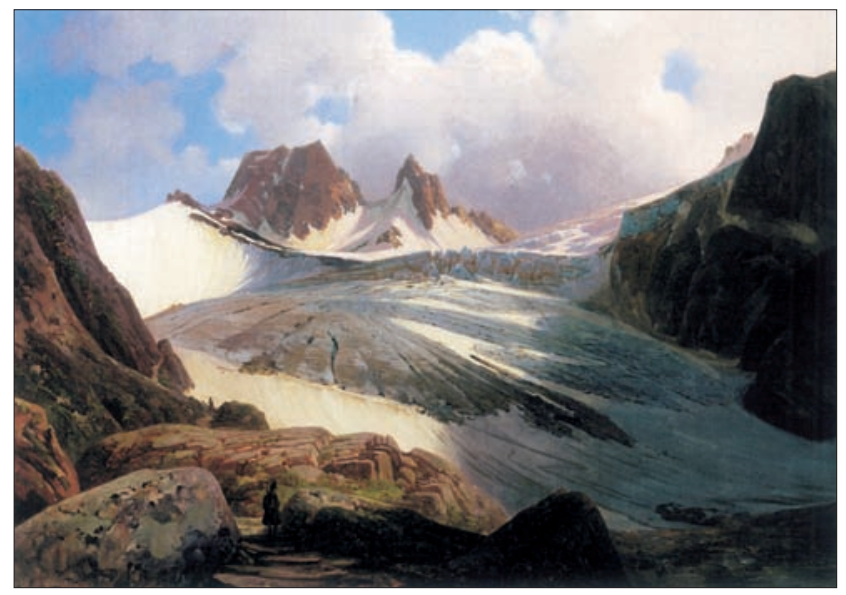

Der Gletschervorstoß um 1820 erregte aber auch in anderen historischen Dokumenten Aufsehen. Klagen der Pinzgauer und Pongauer Bauern über Missernten als Folge der kalten Sommer der 1810er Jahre führten zu einer systematischen Beschreibung der Klima- und Gletscherveränderungen dieser Region in den so genannten Kreisamts-Präsidialakten Nr.84 bis 89 (siehe Slupetzky und Slupetzky, 1995). So wird über wesentlich kleinere Gletscherstände im ausgehenden 18. Jahrhundert berichtet und von einem markanten Gletschervorstoß danach.

Abb. 5: Karte des Bergbaureviers am Rauriser Goldberg von Franz Posepny (Posepny, 1879)

Fig. 5: Map of the mining area at Rauriser Goldberg (Posepny, 1879).

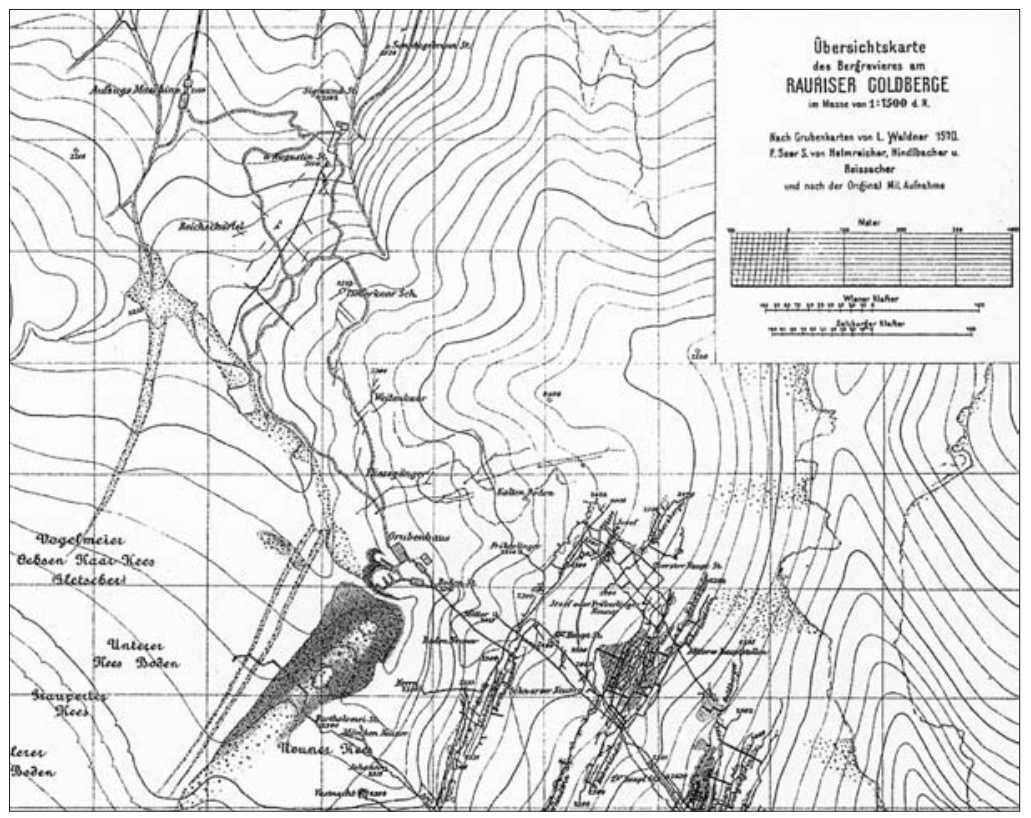




\section{Längenmessungen}

Informationen über Längenänderungen an Gletschern datieren in den Alpen bis ins 19. Jahrhundert zurück, für einzelne Gletscher bis ins 18. Jahrhundert. Einen wesentlichen Aufschwung hat die Messung der Längenänderungen an den Gletschern der österreichischen Alpen durch eine Initiative des Alpenvereins im ausgehenden 19. Jahrhundert erhalten. Durch diese Initiative wurde innerhalb kurzer Zeit eine recht umfangreiche Stichprobe an gemessenen Gletschern erreicht (siehe Abbildung 6), wobei auch die Methode der Messung recht gut genormt wurde. Diese Messreihen besitzen mittlerweile eine beachtliche Länge mit einer zeitlichen Auflösung von 1 Jahr.

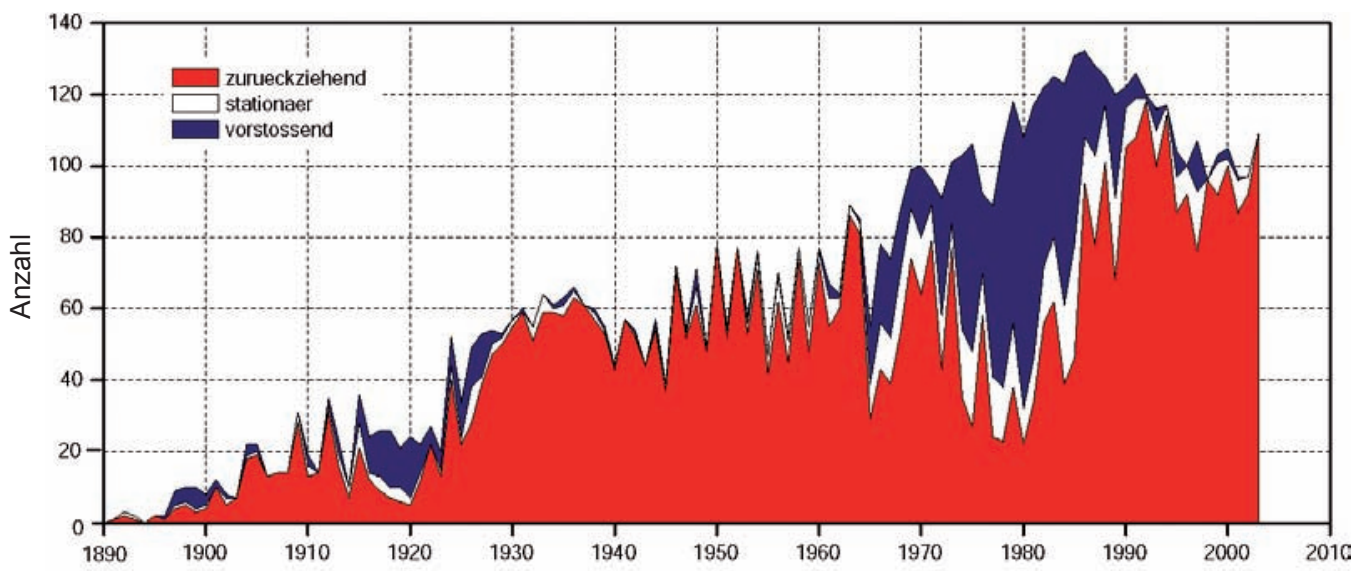

Abb. 6: Längenmessungen an österreichischen Gletschern (Archiv des Österreichischen Alpenvereins)

Fig. 6: Length measurements of Austrian glaciers (archive of the Austrian Alpine Club); retreating (red), stationary (white), advancing (blue).

Die aus Längenänderungsmessungen ableitbaren Proxyinformationen enthalten neben der Massenbilanz (und damit dem Klima) auch die Eisdynamik. Die Längenänderung ist somit eine indirekte verzögerte Reaktion eines Gletschers auf eine Klimaänderung. Auf Grund der Kontinuität muss für eine Längenänderung $\delta \mathrm{L}$ verursacht durch eine Massenbilanzänderung $\delta \mathrm{b}$ nach einer Anpassungszeit $\mathrm{t}_{\mathrm{a}}$ folgendes gelten (Nye, 1960):

$\mathrm{d} L=\frac{L_{0} \cdot \mathrm{d} b}{b_{t}} \quad \begin{aligned} & \text { Dabei ist } \mathrm{b}_{\mathrm{t}} \text { ist die Ablation an der Gletscherzunge. Die Gleichung } \\ & \text { sagt aus, dass aus Längenänderungen eines Gletschers, bei Beachtung } \\ & \text { seiner Anpassungszeit, die Massenbilanz quantitativ bestimmt }\end{aligned}$ 
werden kann. Die Anpassungszeit eines Gletschers kann aus dem Verhältnis zwischen maximaler Eisdicke $h_{\max }$ und Ablation an der Gletscherzunge $b_{t}$ abgeschätzt werden (Johannesson et al., 1989).

$$
t_{a}=\frac{h_{\max }}{b_{t}}
$$

Für die Pasterze mit einer maximalen Eistiefe von ca. 300m und einer Ablation von $10 \mathrm{~m}$ im Zungenbereich ergibt sich aus dieser Beziehung eine Anpassungszeit von ca. 30 Jahren. Eine Interpretation der Längenänderungen eines Gletschers im Hinblick auf Lufttemperatur- und Niederschlagsänderungen verlangt daher die Untersuchung über hinreichend lange Zeitintervalle oder für quantitative Untersuchungen mit hoher zeitlicher Auflösung die Anwendung eines kombinierten Massenbilanzund Eisdynamikmodells (z.B, Oerlemans 2005).

Matulla et al. (2005) haben die Längenänderungen der Alpengletscher für eine qualitative Plausibilitätsprüfung von statistisch bestimmten „Outstanding Periods“ in Klimazeitreihen des HISTALP Datensatzes und eines Globalen Klimamodells verwendet. Es konnte gezeigt werden, dass die detektierten Outstanding Periods der Klimazeitreihen sich gut mit Gletschervorstößen beziehungsweise Gletscherrückzügen decken (siehe Abbildung 7). Eine quantitative Überprüfung dieser Ergebnisse hätte jedoch eine Koppelung mit einem Gletschermodell (Massenbilanz und Gletscherbewegung) verlangt.
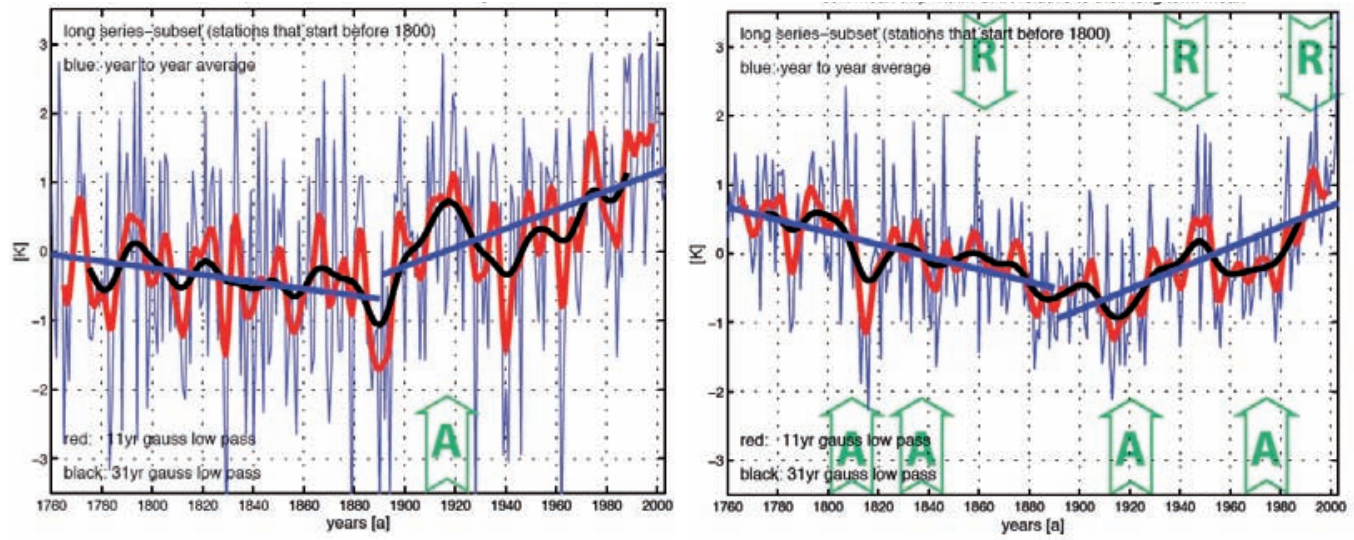

Abb. 7: Verlauf der Luftemperatur im Winter (Dezember bis Februar, links) und Sommer (Juni bis August, rechts) im Alpenraum im Vergleich zu Gletschervorstößen (A) und Gletscherrückzїgen (R); (Temperaturdaten vom HISTALP Datensatz obne "early instrumental correction", Blaue Kurve: Einzelwerte, Rote Kurve:11-jährig gefilterte Werte, Schwarze Kurve: 31-jährig gefilterte Werte).

Fig. 7: Winter (left, January-February) and summer (June-August) temperature series in the Alpine region (Winter (December-February, left) and summer (June-August, right) temperatures in the Alps in comparison to glacier advances (A) and glacier retreats (R); (temperature data from HISTALP data base without the "early instrumental correction", blue curves: single values, red curves: 11 years filtered values, black curves: 31 years filtered values). 
Oerlemans (2005) hat eine weltweite Stichprobe von Gletscherlängenänderungen dazu verwendet, um mittels eines kombinierten Massenbilanz- und Eisbewegungsmodells die Lufttemperaturen für verschiedene Regionen zu rekonstruieren. Als wesentliche Vereinfachung hat er den Einfluss des Niederschlags vernachlässigt und die Lufttemperatur als einzige Klimaeinflussgröße für die Massenbilanz angesetzt. Die berechneten Proxytemperaturen zeigen teilweise eine gute Übereinstimmung mit den Zeitreihen aus instrumentellen Messungen. Eine genauere Betrachtung für den Alpenraum (Abbildung 8) zeigt jedoch, dass insbesondere vor 1900 signifikante Unterschiede zu den HISTALP Temperaturreihen bestehen. Daraus kann man folgern, dass das verwendete Gletschermodell nicht in der Lage zu sein scheint, die Massenbilanz der Gletscher über einen so langen Zeitraum hinreichend genau zu modellieren.

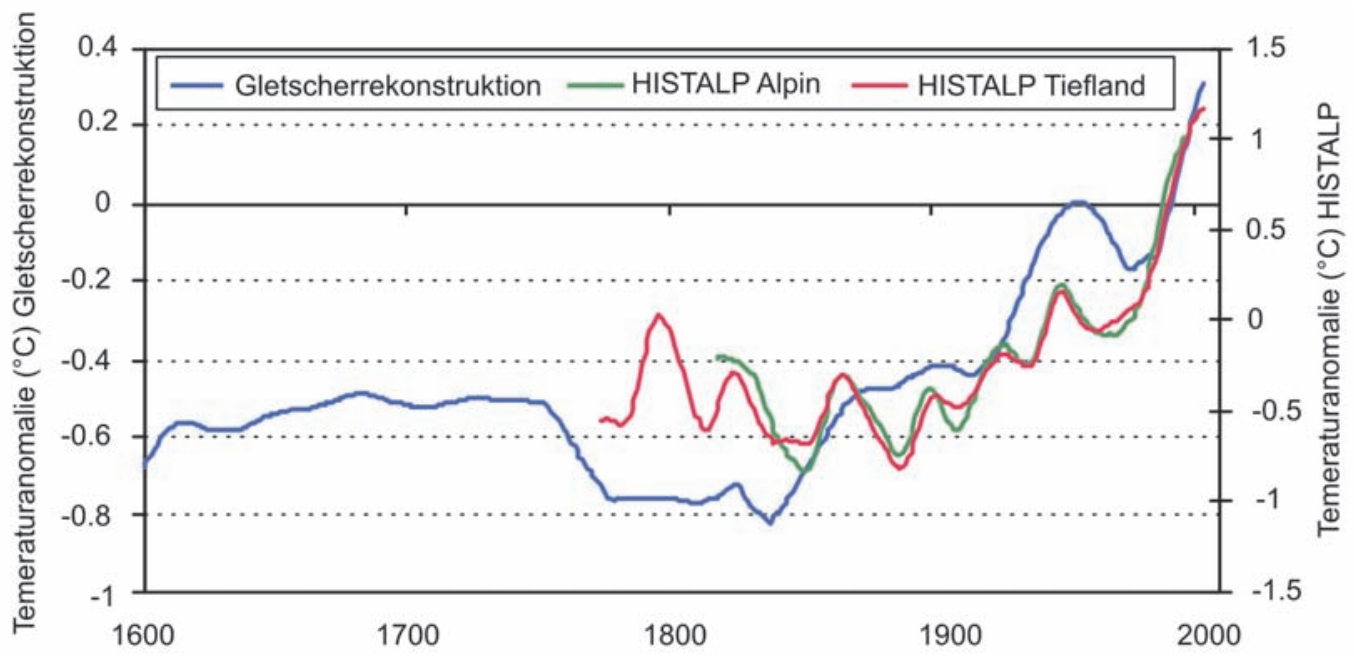

Abb. 8: Vergleich der aus Gletscherlängen rekonstruierten Temperatur (Oerlemans, 2005) mit den HISTALP Temperaturen für den Alpenraum. Berugsperiode 1901-2000, HISTALP Reihen mit „Early-Instrumental Correction", siehe Böhm, dieser Band)

Fig. 8: Comparison of temperatures reconstructed from glacier lengths (Oerlemans 2005) with HISTALP temperatures with early instrumental period correction (Böhm, this volume) for the Alpine Region (reference period 1901-2000); blue: reconstructed; green: HISTALP alpine; red: HISTALP lowland. 


\section{Volumsänderungen}

Die Ableitung von Massenbilanzdaten durch den Vergleich von Höhenlinienplänen beziehungsweise digitalen Höhenmodellen hat unter dem Bergriff ,geodätische Methode der Massenbilanzbestimmung" Eingang in die Glaziologie gefunden. Die Bestimmung der Gletscheränderungsvolumina aus Höhenlinienplänen ist nicht trivial und verlangt einige geometrische Annäherungen, die erstmals durch die Arbeit von Finsterwalder (1953) sehr gut gelöst wurden. Diese Methode basiert auf einer analogen Auswertemethode, wobei Flächen aus den Karten ausgemessen werden. Mit der Einführung digitaler Höhenmodelle wurden diese und ähnliche Methoden von digitalen Auswertemethoden abgelöst. Bei diesen Methoden werden die Volumendifferenzen durch Diskretisierung in Höhendifferenzen in einem räumlich hinreichend aufgelösten Raster berechnet. Die direkte Vergleichbarkeit zwischen den älteren analogen mit den gegenwärtigen digitalen Methoden ist jedoch oft nicht gegeben, was zu systematischen Unterschieden führt (z.B. Schöner und Schöner 1995). In den letzten Jahren werden digitale Höhenmodelle von Gletschern vermehrt durch Laserscanningmethoden erarbeitet.

Auch wenn meist von der geodätischen Methode der Massenbilanzbestimmung gesprochen wird, ist trotzdem zu beachten, dass es sich dabei um eine Methode zur Bestimmung von Volumsunterschieden handelt. Da keine Messwerte der Dichte der abgeschmolzenen Volumina vorliegen, wird im Allgemeinen als Näherungswert die Dichte von Eis für die Umrechnung der Volumsunterschiede in Massenunterschiede angesetzt. Diese Annäherung ist meist gut erfüllt, in Sonderfällen können jedoch aber auch größere Abweichungen bestehen. Neben dieser Einschränkung durch die fehlende Dichte der abgeschmolzenen Volumina haben die geodätischen Massenbilanzdaten auch den Nachteil, dass sie nur Aussagen über längere Zeitspannen ermöglichen. Ihr besonderer Wert liegt jedoch darin, dass sie Messwerte bis in die ausgehende Kleine Eiszeit liefern.

Bestimmungen von Volumsänderungen der Gletscher sind seit den ersten genauen großmaßstäbigen kartographischen Aufnahmen der Alpen möglich. Es ist aus heutiger Sicht überraschend, wie früh bereits hochwertige großmaßstäbige Karten von manchen Gletscher angefertigt wurden. Brunner $(1990,2000)$ gibt einen guten Überblick über diese frühen kartographischen Meisterleistungen der Hochgebirgskartographie. Herausragend sind dabei die Arbeiten der Gebrüder Schlagintweit (1850) oder die Arbeit von K.v.Sonnklar (1883) sowohl für das Gebiet der Ötztaler Alpen als auch für die Hohen Tauern, wodurch bereits für das beginnende 
Klimawandel in Österreich
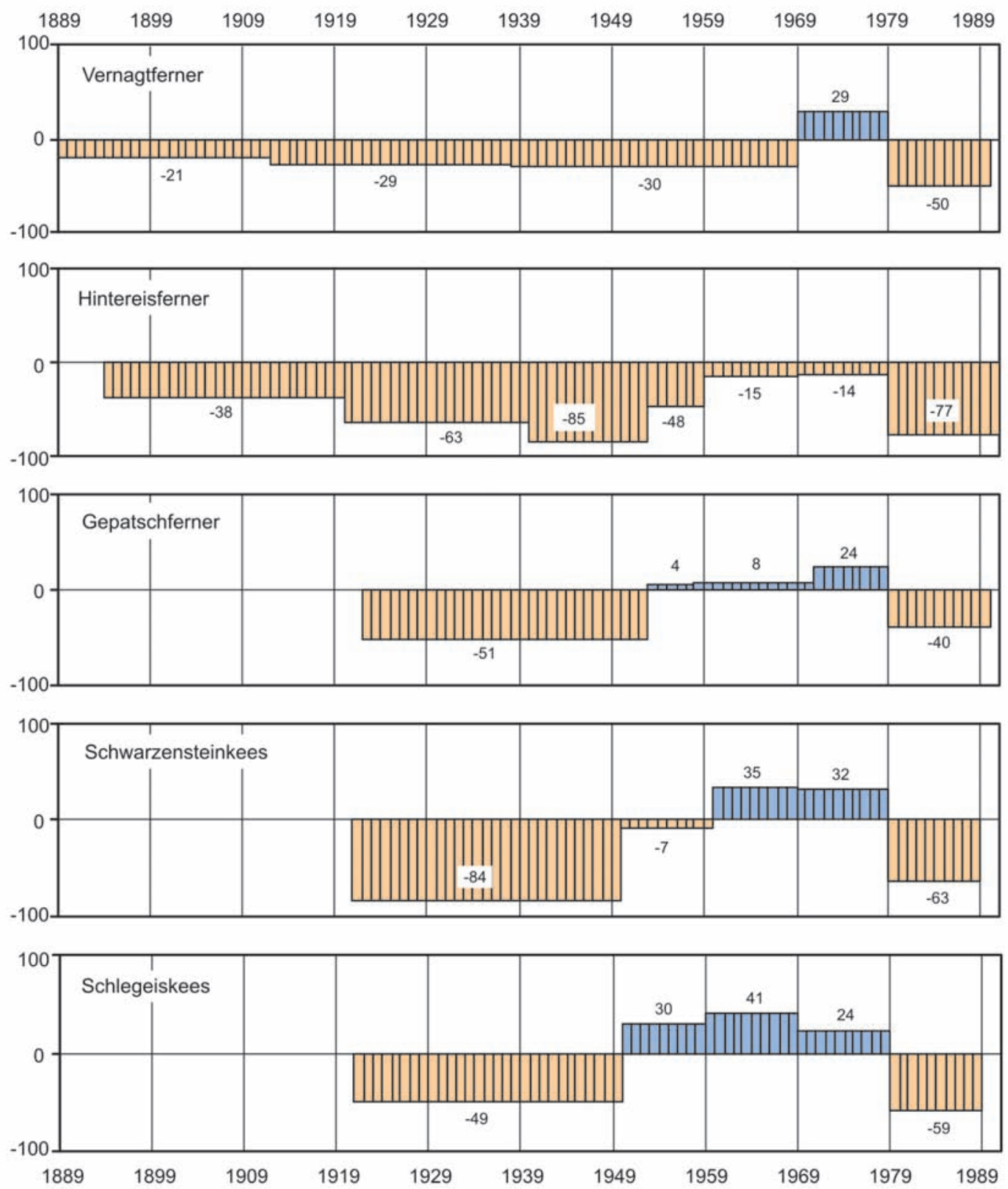

Abb. 9: Volumsänderungen von Gletschern der österreichischen Alpen (Daten von Rentsch et al., 2006)

Fig. 9: Volumn changes of Austrian Glaciers (Data from Rentsch et al., 2006). 
18. Jahrhundert der Zustand der Alpengletscher dokumentiert wurde. Auch wenn die absoluten Fehler dieser Karten recht groß sind, ist doch eine erste quantitative Abschätzung durch Vergleich mit späteren Karten möglich.

In Abbildung 9 sind die Volumsunterschiede ausgewählter Gletscher der österreichischen Alpen seit den ersten Kartenaufnahmen dargestellt. Die ausgewählten Gletscher befinden sich in den Ötztaler Alpen, den Zillertaler Alpen und in den Hohen Tauern - decken also das Gesamtgebiet der österreichischen Alpen recht gut ab. Man sieht recht deutlich, dass markante Klimaphasen sich in den Volumsänderungsdaten deutlich widerspiegeln. So zeigen viele Gletscher einen Gletschervorstoß um 1920 und um 1980, sowie einen ausgeprägten Rückzug nach 1850, in den 1950er Jahren und in der Periode seit 1980.

Die Auswertung von Volumsunterschieden von Gletschern erlaubt heutzutage die Erfassung von einer sehr großen Anzahl von Gletschern. Dieser Ansatz wurde für Österreich im Rahmen des Gletscherinventars verfolgt. Dabei wurden photogrammetrische Auswertungen von systematischen Befliegungen der österreichischen Gletscher im Jahr 1969 (1tes Inventar) mit Befliegungen in den späten 1990er Jahren verglichen. Die Ergebnisse liegen mittlerweile vor und wurden z.B. im Hydrologischen Atlas Österreichs (Gattermayr et al., 2005) publiziert.

\section{Massenbilanzmessungen}

Direkte Messreihen der Massenbilanz sind vergleichsweise kurz und gehen für die österreichischen Gletscher nicht vor 1950 zurück. Ein internationaler Vergleich zeigt, dass in anderen Hochgebirgsregionen die Messreihen kaum länger sind (die längste Messreihe liegt vom Storglaciären in Schwedisch Lappland vor, die bis 1948 zurückreicht). Massenbilanzmessungen sind sehr aufwändig und wurden erst durch den Aufschwung der Glaziologie nach dem 2. Weltkrieg ermöglicht.

Die Massenbilanzbestimmung nach der direkten glaziologischen Methode wurde mehrfach beschrieben (z.B. Hoinkes 1970). Im Zusammenhang mit den hier beschriebenen Klimainformationen, abgeleitet aus Gletscheränderungen, sind die Massenbilanzmessungen sowohl für das Verständnis als auch für die Entwicklung entsprechender physikalischer Modelle des Zusammenhanges KlimaänderungGletscherverhalten wichtig. Eine Übersichtsdarstellung der Massenbilanzmessungen an österreichischen Gletschern erfolgte in Schöner et al. (2000) und in Schöner und Böhm (2007). Die vorliegenden langjährigen Messreihen sind in Abbildung 10 zusammengefasst. Deutlich ist in der Abbildung die höhere Variabilität der Massenbilanzen in den Hohen Tauern im Vergleich zu den Ötztaler Alpen erkennbar. 

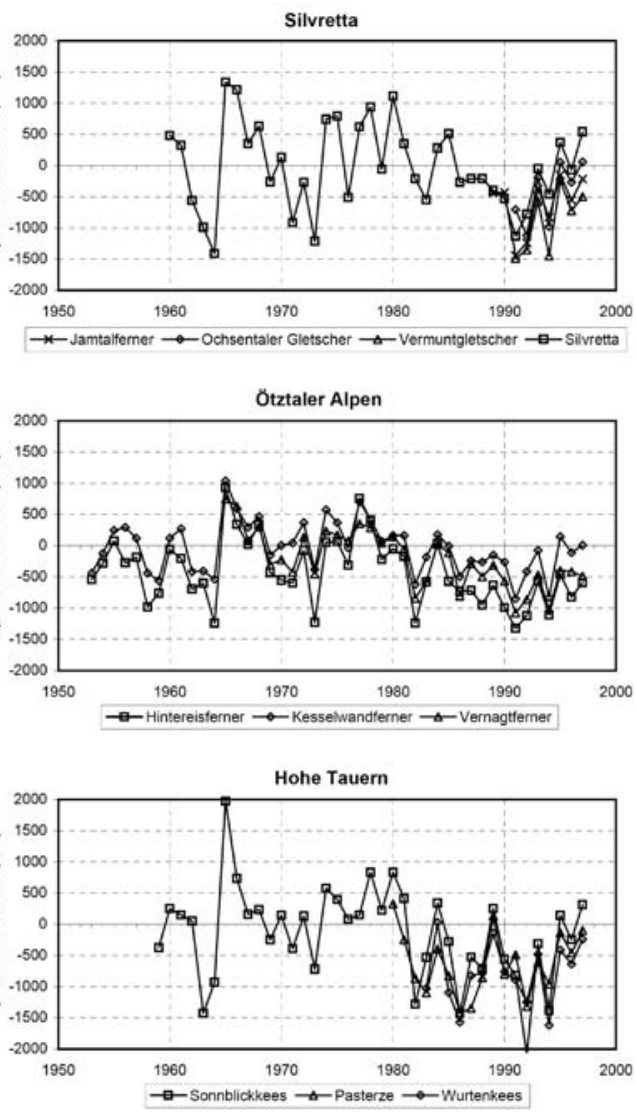

Abb. 10: Ergebnisse von Massenbilanzmessungen an österreichischen Gletschern (aus Schöner et al., 2001)

Fig. 10: Results of mass balance measurements of Austrian galciers (from Schöner et al. 2001).

Reaktion der Schneegrenze (Equilibrium Line Altitude ELA) auf Klimaänderungen

Die mittlere Höhe der Gleichgewichtslinie ELA eines Gletschers (oft auch als Schneegrenze bezeichnet) und das Flächenverhältnis des Akkumulationsgebietes Sc zu Gesamtgletscherfläche S sind sehr hoch mit der Massenbilanz korreliert. Jedoch ist auch dieser Zusammenhang für jeden Gletscher unterschiedlich. Insbesondere das Flächenverhältnis Sc/S lässt sich leicht aus Luftbildern oder Satellitenaufnahmen bestimmen. Für die Bestimmung der ELA ist zusätzlich noch ein digitales Höhenmodell notwendig (siehe z.B. Zemp, 2006).

Kuhn (1980) hat den Zusammenhang zwischen dem Klima, der Schneegrenze und der Massenbilanz eines Gletschers dargelegt und die Verwendbarkeit der Schneegrenze als Klimaindikator demonstriert. Nach einigen Vereinfachungen kommt er 
zu folgenden berechneten Werten einer Klimaänderung bei einer Änderung der ELA um 100m aufwärts:

entweder Abnahme der Jahresakkumulation um $400 \mathrm{~kg} \mathrm{~m}^{-2}$

oder Abnahme der Strahlungsbilanz um $1.33 \mathrm{MJ} \mathrm{m}^{-2} \mathrm{~d}^{-1}$

oder Zunahme der Lufttemperatur um $0.8^{\circ} \mathrm{C}$

Die notwendige Zunahme der Lufttemperatur um $0.8^{\circ} \mathrm{C}$ deckt sich dabei nicht mit einem durchschnittlichen vertikalen Temperaturgradienten in der Atmosphäre, da für die Akkumulation auch eine Zunahme mit der Höhe angenommen wurde. Mit Zahlenwerten der Lufttemperatur, des Niederschlags und der Strahlungsbilanz können dann systematische Unterschiede der ELA zwischen dem Nordrand der Ostalpen (ca. $2600 \mathrm{~m}$ ) und den Zentralalpen (ca. 3000 m) erklärt werden. In Kuhn (1989) wurde der Zusammenhang zwischen der ELA und dem Klima mit einem verfeinerten Modell erfasst. Die berechneten Werte zur Reaktion der ELA auf eine Klimaänderung sind deutlich verschieden zu Kuhn (1980), wobei Rückkoppelungen zwischen den einzelnen Klimakenngrößen nicht berücksichtigt sind:

Anstieg der ELA um 130m bei Zunahme der Strahlungsbilanz um $1 \mathrm{MJ} \mathrm{m}^{-2} \mathrm{~d}^{-1}$

Anstieg der ELA um $65 \mathrm{~m}$ bei Zunahme der Lufttemperatur um $1{ }^{\circ} \mathrm{C}$

Anstieg der ELA um 35m bei Zunahme der Jahresakkumulation um $100 \mathrm{~kg} \mathrm{~m}^{-2}$

Diese Werte entsprechen eher den Ergebnissen von anderen Studien (z.B. Maisch, 1987). Tatsächlich wird eine Änderung der Schneegrenze aus dem Zusammenwirken von Änderungen aller oben beschriebenen Größen verursacht. Auch beziehen sich die obigen Ergebnisse auf Gleichgewichtszustände, die jedoch bei vielen Gletschern nicht erreicht werden. Patzelt (1973) hat für die Venedigergruppe die Änderung der Schneegrenze zwischen den Gletscherständen von 1850 und 1920 für 9 Gletscher untersucht und Werte zwischen $10 \mathrm{~m}$ und $70 \mathrm{~m}$ gefunden (Mittel $26 \mathrm{~m}$ ). Diese Änderung kann jedoch mit den oben angeführten Werten einer Klimaänderung kaum begründet werden und spricht dafür, dass die Gletscher keinen Gleichgewichtszustand erreicht hatten.

Maisch $(1987,1988,1993)$ hat die Änderung der Schneegrenze systematisch für die Gletscher Graubündens untersucht und einen Anstieg um $77 \mathrm{~m}$ (Mittelwert über 528 Gletscher) für die Periode 1850 bis 1973 gefunden. Ein Vergleich mit der Sommertemperaturkurve der Alpen legt nahe, dass auch diese Änderung der ELA nicht aus der Änderung der Lufttemperatur alleine begründet werden kann. Maisch (1993) gibt als Äquivalent zum Anstieg der ELA um 100m eine Temperaturzunahme für den Sommer von $0.6^{\circ} \mathrm{C}$ für die Periode 1850 bis 1993 sowohl für die Region Morteratsch als auch Roseg an. Die HISTALP Kurve der Sommertemperatur zeigt 
dagegen kaum einen Unterschied, wobei jedoch die Frage des notwendigen Mittelungszeitraumes nicht beantwortet werden kann. Als Schlussfolgerung kann abgeleitet werden, dass derart einfache Modelle der ELA Verschiebung (die nur eine Temperaturänderung berücksichtigen) kaum in der Lage sind, die Änderung des Klimas zu beschreiben.

Aufbauend auf Ohmura (1992) hat Zemp (2006) ein statistisches Modell entwickelt, das den Zusammenhang zwischen der ELA, der Lufttemperatur im Sommer und dem Winterniederschlag aus gemessenen Daten beschreibt. In weiterer Folge verwendet er diesen Zusammenhang, um aus ELA Verschiebungen, die sich aus Temperatur- und Niederschlagsänderungen ergeben, eine klimatisch mögliche Akkumulationsfläche zu berechnen (Abbildung 11). Eine unkritische Verwendung eines konstanten Sc/S Verhältnisses (ein häufig verwendeter Ansatz um die ELA zu bestimmen) für die Rekonstruktion der Klimaänderung lehnt er, auf Grund der tatsächlich sehr großen Spannweite dieses Verhältnisses, ab.

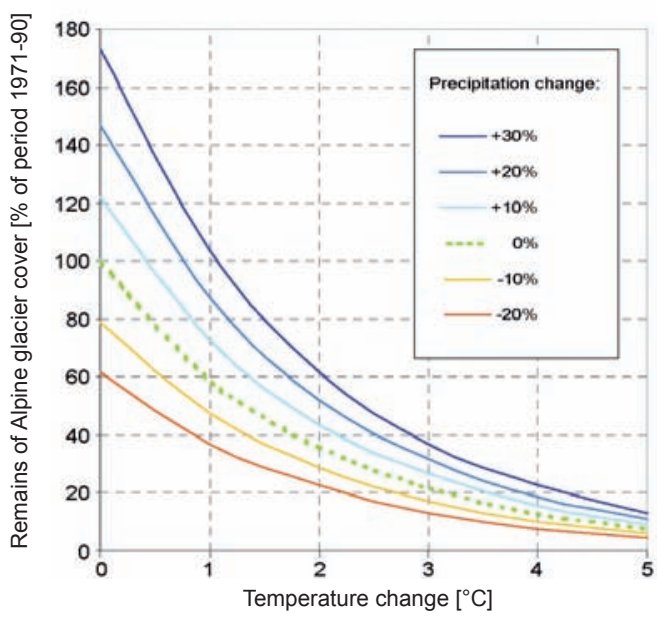

Schlussfolgerungen

Abb. 11: Modellierte Alpenvergletscherung (klimatisches Akeumulationsgebiet) unter Annahme eines Anstieges der Sommertemperatur um +1 bis $+5{ }^{\circ} \mathrm{C}$ und/oder einer Änderungen des Jahresniederschlages. 100\% entsprechen der Vergletscherung der Referenzperiode (1971-90). Die punktierte Linie entspricht einer Temperaturänderung obne Niederschlagsänderung, während die anderen Linien kombinierte Temperatur- und Niederschlagsänderungen darstellen (Zemp, 2006).

Fig. 11. Modelling of the glaciation of the Alps (climatic accumulation area) under the assumption of summer temperature increases by +1 to + $5^{\circ} \mathrm{C}$ and/or changes in anual precipitation. One hundred $\%$ correspond to the glaciation in the reference period (1971-90). The dotted line corresponds to a temperature change without precipitation change while the other lines represent a combination of temperature and precipitation changes (Zemp 2006).

Die Ableitung von Klimainformationen aus Gletscheränderungsgrößen, im Sinne durchgehender Proxyreihen mit hoher zeitlicher Auflösung, ist schwierig und wurde für die Alpengletscher bis jetzt nur ansatzweise durchgeführt. Längere Zeitreihen über Gletscheränderungen in hoher zeitlicher Auflösung liegen nur von Gletscherlängen vor. Die Bestimmung von Klimaproxyreihen aus Gletscherlängen verlangt eine aufwändige Modellierung. Für diese Modelle (Massenbilanz, ELA) werden oft 
statistische Ansätze verwendet, die an Messdaten entsprechend kalibriert werden. Die zeitliche Übertragbarkeit dieser statistischen Zusammenhänge, gerade über sehr lange Zeiträume, ist jedoch unsicher.

Ein wesentlicher Wert der Daten über Gletscheränderungen liegt in ihrem Potential, mittels Modelle (Massenbilanz und ev. Gletscherdynamik) die Plausibilität von Klimazeitreihen testen zu können. Liefern Temperatur- und Niederschlagsreihen die korrekten Massenbilanzen (die man aus Volumsdifferenzen bestimmen kann) oder die korrekten Gletscherlängen, dann können diese Zeitreihen als sehr plausibel bezeichnet werden.

\section{Literatur}

Auer I, Böhm R, Jurkovic A, Lipa W, Orlik A, Potzmann R, Schöner W, Ungersböck M, Matulla C, Briffa K, Jones PD, Efthymiadis D, Brunetti M, Nanni T, Maugeri M, Mercalli L, Mestre O, Moisselin J-M, Begert M, Müller-Westermeier G, Kveton V, Bochnicek O, Stastny P, Lapin M, Szalai S, Szentimrey T, Cegnar T, Dolinar M, Gajic-Capka M, Zaninovic K, Majstorovic Z, Nieplova E, 2007. HISTALP - Historical instrumental climatological surface time series of the greater Alpine region 1760-2003. International Journal of Climatology, 27: 17-46.

Brunner K. 1990. Gletscherdarstellungen in alten Karten. Cartographica Helvetica, Heft 2, 9-19.

Brunner K. 2000. Erste großmaßstäbige Gletscherkarten unter besonderer Berücksichtigung der Hohen Tauern. Festschrift Heinz Slupetzky zum 60. Geburtstag. Salzburger Geographische Arbeiten, Band 36, 7-22.

Finsterwalder R. 1953. Die zahlenmäßige Erfassung des Gletscherrückganges an Ostalpengletschern. Zeitschrift für Gletscherkunde und Glazialgeologie, II, Heft 2,.

Gattermayr W., M. Kuhn, V. Weilguni. 2005. Schneemessstellen und beobachtete Gletscher. Hydrologischer Atlas Österreichs, Wien

Hammer N. 1993. Rekonstruktion einer 100jährigen Reihe der Gletschermassenbilanz. Zeitschrift für Gletscherkunde und Glazialgeologie, Band 29, Heft 1, 73-87.

Hermann A. und M. Kuhn. 1990. Schnee und Eis. In: Baumgartner A. und H.J. Liebscher (Hrsg.). Lehrbuch der Allgemeinen Hydrologie. Bornträger, Berlin

Hoinkes H. 1970. Methoden und Möglichkeiten von Massenhaushaltsstudien auf Gletschern - Ergebnisse der Messreihe Hintereisferner 1953-1968. Zeitschrift für Gletscherkunde und Glazialgeologie, Band VI, 37-90.

Hoinkes H. und R. Steinacker. 1975. Zur Parametrisierung der Beziehung Klima-Gletscher. Rivista Italiana di Geofisica Vol. I, 97-104.

Johannesson T., C. Raymond, E. Waddington. 1989. Time-scale adjustment of glaciers to changes in mass balance. Journal of Glaciology, Vol. 35, No. 121, 355-369.

Kuhn M. 1980. Die Reaktion der Schneegrenze auf Klimaschwankungen. Zeitschrift für Gletscherkunde und Glazialgeologie, Band 16, Heft 2, 241-254.

Kuhn M. 1989. The response of equilibrium line altitude to climate fluctuations: theory and observations. In: Oerlemans J. (Glacier Fluctuations and Climate Change). Glaciology and Quaternary Geology, 407-417.

Kuhn M. 2004. Die Reaktion der österreichischen Gletscher und ihres Abflusses auf Änderungen von Temperatur und Niederschlag. Österreichische Wasser- und Abfallwirtschaft 56(1-2), 1-7 


\section{Klimawandel in Österreich}

Maisch M. 1987. Die Gletscher um 1850 und HEUTE im Bündnerland und in den angrenzenden Gebieten: Untersuchungen zur Höhenlage, Veränderung und räumlichen Struktur von Schneegrenzen. Geographica Helvetica 1987, Heft 2, 127-145.

Maisch M. 1988. Die Veränderungen der Gletscherflächen und Schneegrenzen seit dem Hochstand von 1850 im Kanton Graubünden (Schweiz). Zeitschrift für Geomorphologie, Suppl-Bd. 70, 113-130.

Maisch. M. 1993. Gletscherschwundphasen im Zeitraum des ausgehenden Spätglazials (Egesen-Stadium) und seit dem Hochstand von 1850 sowie Prognosen zum zukünftigen Eisrückgang in den Alpen. Gletscher im ständigen Wandel, Jubiläums-Symposium der Schweizerischen Glescherkommission, Publikationen der Schweizerischen Akademie der Naturwissenschaften 6, 81-100.

Matulla C, Auer I, Böhm R, Ungersböck M, Schöner W., Wagner S, Zorita E. 2005. Outstanding past decadal-scale climate events in the Greater Alpine Region analysed by 250 years data and model runs. GKSS-Report 2005/4

Nye J. 1960. The response of glaciers and ice-sheets to seasonal and climatic changes. Proceedings of the Royal Society of London, Series A (256), 559-584.

Oerlemans, J. 2005. Extracting a climate signal from 169 glacier records. Science, 308(5722), 675-677.

Ohmura A., P. Kasser, M. Funk. 1992. Climate at equilibrium line of glaciers. Journal of Glaciology $38,397-411$.

Ohmura A. 2001. The physical basis for the temperature based melt-index method. Journal of Applied Meteorology 40/4, 753-761

Patzelt G. 1973. Die neuzeitlichen Gletscherschwankungen in der Venedigergruppe (Hohe Tauern, Ostalpen). Zeitschrift für Gletscherkunde und Glazialgeologie, Band IX, Heft 1-2, 5-57.

Posepny F. 1879. Die Goldbergbaue der Hohen Tauern mit besonderer Berücksichtigung des Rauriser Goldbergwerkes. Archiv für praktische Geologie 1, Wien

Rentsch H., K. Eder, T. Geiss. 2006. Der Gletscherrückgang in den Ostalpen in der letzten Dekade des 20. Jahrhunderts. Zeitschrift für Gletscherkunde und Glazialgeologie, Band 39, 65-74.

Schlagintweit H. und A. Schlagintweit. 1850. Untersuchungen über die Physicalische Geographie der Alpen. Leipzig.

Schöner M. und W. Schöner. 1995. Determination of changes in volume of Gaasbreen using different geodetic methods. Zeitschrift für Gletscherkunde und Glazialgeologie, Band 31, 85-91.

Schöner W., Auer I. and Böhm R. 2000. Climate variability and glacier reaction in the Austrian eastern Alps. Annals of Glaciology, Vol. 31, 31-38.

Schöner W., Böhm R. 2007. A statistical mass-balance model for reconstruction of LIA ice mass for glaciers in the European Alps. Annals of Glaciology, Vol. 46, 161-169.

Slupetzky H. und N. Slupetzky. 1995. "Betref des Wachsthums der Kletscher und Kälterwerdung des Klimas". Slazburger Geographische Materialien 23, 42 Seiten.

Sonnklar K.v. 1883. Die Gebirgsgruppe der Hohen Tauern. Beck’sche Universitätsbuchhandlung, Wien.

Wagenbach D. and 7 others. 1998. Environmental and climate records from high elevation Alpine glaciers (ALPCLIM). In: European Climate Science Conference, 19-23 October 1998, Vienna, Proceedings, Vienna, Austrian Ministry of Science and Transport

Zemp M. 2006. Glaciers and climate - spatio-temporal analysis of glacier fluctuations in the European Alps after 1850. Schriftenreihe Physische Geographie Glazialgeologie und Geomorphodynamik, 49, Zürich. 


\title{
Klimarekonstruktion der instrumentellen Periode - Probleme und Lösungen für den Großraum Alpen
}

\author{
Reinhard Böhm \\ Zentralanstalt für Meteorologie und Geodynamik, Wien
}

\begin{abstract}
Climate reconstruction of the instrumental period - problems and solutions for the greater Alpine region

Two recent activities of the climate variability working group at the Austrian weather service (ZAMG) are chosen to demonstrate the extraordinary potential of the instrumental record existing in the greater Alpine region and to highlight the risks arising with the uncritical use of original instrumental climate information. First we deal with the correction of a systematic warm bias found in the early instrumental summer temperatures prior the 1860s. This exercise is a successful step towards real climate information with a reduced share of yet undetected or not yet removable non-climatic information (random noise but also systematic biases). The second example describes a yet not successfully solved problem: the homogenization of daily or subdaily climate series. This matter is particularly urgent as the ongoing discussion on the change of extreme events in frequency and/or intensity needs proper data, which are presently not available. Here some regionally limited attempts are shown and discussed.
\end{abstract}

\section{Einleitung}

Gerade im Zusammenhang mit dem in diesem Band im Vordergrund stehenden Thema der Klimarekonstruktion mit Hilfe von indirekten (Proxy-) Daten mag es vielleicht überraschen, dass hier der Begriff „Rekonstruktion“ auch für direkt gemessene Klimadaten Verwendung finden soll. Es herrscht ja im Allgemeinen die Ansicht, gemessene Klimadaten seien a priori „die Wahrheit“, an der die Klimamodelleure ihre Ergebnisse messen und mit denen die Klimarekonstrukteure ihre indirekten Informationen kalibrieren. Dass dem nicht ganz so ist, soll dieser Beitrag mit zwei Beispielen vermitteln. Ein Detailproblem aus der frühen instrumentellen Periode wird zum Anlass genommen, den Weg von Originaldaten des Klimaelements Lufttemperatur aus der Zeit vor 1860 über eine homogenisierte Fassung eines Monatsdatensatzes für den Großraum Alpen aus 2007 zu der aktuellen korrigierten Fassung von 2008 zu zeigen. Dieses erste Problem kann als gelöst angesehen werden. Ein zweites, das der adäquaten Behandlung langer Klimazeitreihen in täglicher bis subtäglicher Auflösung harrt, noch seiner Lösung und wir können hier nur die Gründe dafür zeigen und Lösungsstrategien für die Zukunft besprechen.

alpine space - man \& environment, vol. 6: Klimawandel in Österreich (C) 2009 iup • innsbruck university press, ISBN 978-3-902571-89-2 


\section{Problem 1: Homogenisierung von Klimadaten in monatlicher Auflösung - der Spezialfall des ,early instrumental bias"}

Gerade der Großraum Alpen zeichnet sich vor allen anderen Gebieten der Erde durch die größte Tradition an lange zurückreichenden Klimadaten aus. Bis zu 250 Jahre in die Vergangenheit reichen die längsten ununterbrochenen Klimareihen - nicht weniger als sechzehn beginnen bereits im 18. Jahrhundert, 32 vor 1850. Natürlich sind historische Messungen aus Zeiten Goethes, Maria Theresias oder Mozarts nicht a priori in vergleichbarer Art gemacht worden, wie es den modernen Standards entspricht. Die daher notwendige Anpassung der historischen Daten an den aktuellen Standard nennt man in der Klimatologie Homogenisierung. Es geht dabei darum, in einer langen Messreihe die echte Klimainformation (das Signal) von einer Reihe anderer Informationen (dem Rauschen) zu trennen, die etwa von Stationsverlegungen, Änderungen der Umgebung (z.B.. die steigende Urbanisierung), technologischen Entwicklungen (Messtechnik) und veränderten statistischen Verarbeitungsstandards stammen.

In den letzten fünfzehn Jahren konnte die Arbeitsgruppe Klimavariabilität der Zentralanstalt für Meteorologie und Geodynamik in Wien einen räumlich dichten (mehrere hundert Einzelstationen), multiplen (mehrere Klimaelemente), langen (längste Reihen zurück bis 1760), qualitätsgeprüften (tausende Inhomogenitäten und Ausreißer entfernt, Datenlücken geschlossen und dadurch eine ursprüngliche Ausfallsrate von 5\% beseitigt) Klimadatensatz erstellen. Der kleinste Zeitschritt des HISTALP-Datensatzes ist ein Monat. Die Daten wurden von zahlreichen (mehr als zwanzig) Providern aus zehn Ländern der Region gesammelt und vorläufig als geschlossener Datensatz in „station-modes“ und in drei verschiedenen „grid-modes“ aufbereitet. Zusatzfinanzierungen zu HISTALP kamen von verschiedenen nationalen und EU-Projekten (CLIVALP, ALOCLIM, ALPCLIM, ALP-IMP). Die ZAMG plant, HISTALP auch in Zukunft als qualitätsorientiertes Klima-Monitoring weiter zu führen. Neben den erwähnten Kontakten mit den Datenprovidern existiert diesbezüglich eine enge Zusammenarbeit mit der Climatic Research Unit der University of East Anglia in Norwich, dem ISAC-Institut des CNR-Bologna und dem Institut für allgemeine und angewandte Physik der Universität Mailand.

Eine HISTALP Gesamt- und Referenzpublikation (Auer et al., 2007) bringt die genauen Details zur Erstellung des Datensatzes und erste Analysen. Mit Jänner 2008 ist ein internes ZAMG-Projekt gestartet, das die HISTALP Daten bis Ende 2007 aktualisiert, reanalysiert, das Messnetz verdichtet und die Daten über die Website der ZAMG leicht und schnell zugänglich machen wird. Eines der in dem Projekt zu erledigenden Probleme war ein vermuteter systematischer Fehler der frühen Temperaturmessungen, der durch unge- 
nügende Abschirmung der Thermometer gegen direkte und indirekte Sonneneinstrahlung entstanden sein könnte. Die Lösung dieses Problems sei hier exemplarisch beschrieben und das Ergebnis kurz vorgestellt.

Abbildung 1 zeigt die vom „EIP-Bias“ (EIP = early instrumental period) betroffenen Stationen zusammen mit dem dichten HISTALP Temperaturmessnetz. Das Sample der Langzeitstationen wird künftig als „LSS“ (long series subset) bezeichnet. Dieser Langzeitdatensatz steht in seiner räumlichen Dichte und Qualität einzigartig da. Nirgendwo sonst gibt es derart viele frühe Klimareihen: von Rudloff (1967) führt für ganz Europa 65 derartiger Langzeitreihen an, allein 32 davon befinden sich im Großraum Alpen und sind Teil des HISTALP Datensatzes.

Abb. 1: Das HISTALP Temperaturmessnetz im Großraum Alpen. Blaue Punkte: 102 Temperaturreiben, die nach 1850 beginnen. Rote Punkte: 32 LSS-Reihen, die zwischen 1760 und 1850 beginnen.

Fig. 1: The HISTALP network of temperature recording stations. Blue points: 102 temperature series starting after 1850; red points: 32 LSSseries starting between 1760 and 1850 .

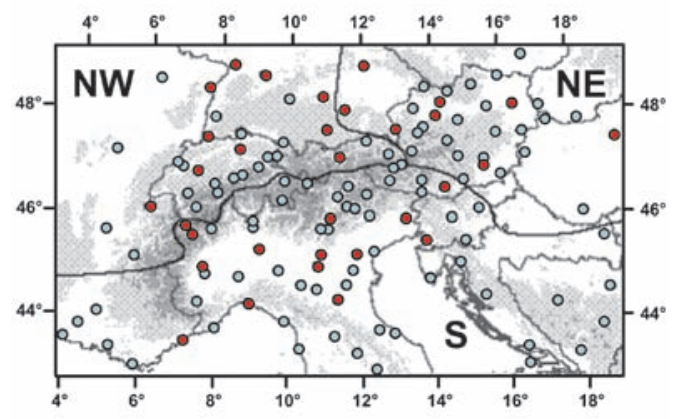

\section{Das Problem}

Vor der Gründung nationaler oder regionaler Wetterdienste, die mit dem Österreichischen Wetterdienst im Jahr 1851 begann (Hammerl at al., 2001) und in den 1870er Jahren im Wesentlichen als abgeschlossen betrachtet werden kann, wurden ein Jahrhundert und länger bereits als Einzelaktivitäten vor allem astronomischer Observatorien Klimazeitreihen geschaffen, die ins 18. Jahrhundert zurückreichen. Zwar schufen diese meist von qualifizierten Wissenschaftlern betriebenen Aktivitäten wertvollste Klimainformationen, jedoch nach der damaligen Messphilosophie und mit der damals bestehenden Messtechnik. Letztere verursachte wenig Probleme, die Thermometer wurden i.a. gut gepflegt, häufig kalibriert (Eisbad bzw. Wasserdampf) und die Ergebnisse sorgfältig festgehalten und meist in Jahrbüchern publiziert. Auch die Metadaten (d.h. Angaben über die Aufstellung der Messgeräte etc.) sind aus der frühen Periode durchaus mit den heutigen vergleichbar - der Betrieb einer Messstation war damals etwas Besonderes und wurde recht genau beschrieben. Die vorhin erwähnte „Messphilosophie“ allerdings war zwischen den LSSStationen wenig bis gar nicht koordiniert, was zu recht unterschiedlichen Aufstellungsarten, 


\section{Klimawandel in Österreich}

Messzeitpunkten und Berechnungsalgorithmen der Mittelwerte führte, die allerdings üblicherweise gut dokumentiert wurden, was für unsere weitere Vorgangsweise von großer Bedeutung war. So konnten die Dauer der EIP für 30 der 32 Stationen des LSS im Großraum Alpen relativ eindeutig eruiert werden. Die frühen instrumentellen Messperioden (EIP) sind im linken Diagramm der Abb. 2 dargestellt und unterscheiden sich von der folgenden eigentlichen instrumentellen Periode (der Wetterdienstperiode) vor allem durch die damals vorherrschende Ansicht, dass es genügt, ein Thermometer an einer nach Norden orientierten Mauer, Fensternische o.ä. zu montieren, um eine Verfälschung durch direkte Bestrahlung durch die Sonne zu vermeiden. In manchen Fällen wurden noch Bretter seitlich oder oberhalb des Thermometers angebracht, da ja im Sommer der Einfall der Sonnenstrahlung auch eine Nordwand trifft, außerdem waren die Gebäude ja nur selten exakt nach Norden

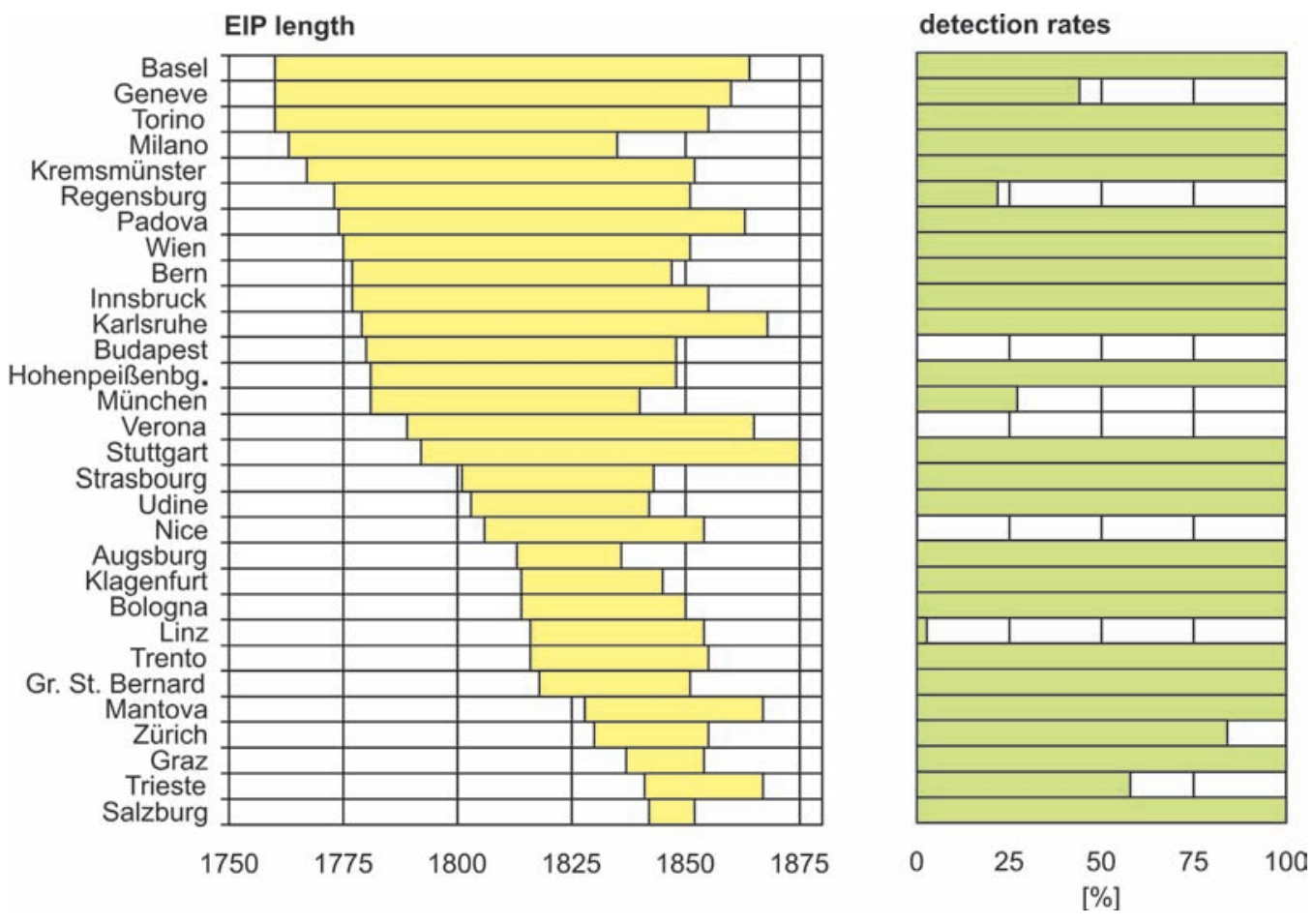

Abb. 2: Links: Die Zeitspannen, für die das EIP Problem einer ungenügenden Abschirmung der Thermometer aus den Stationsgeschichten von 30 der 32 Standorte ermittelt werden konnte. Rechts: Die ,Aufkelärungsquote" für die in der EIP verwendeten Berechnungsalgorithmen für das Temperaturmittel (Böbm et al, 2008).

Fig. 2: Left: The time spans for which the EIP problem of an insufficient temperture shield was detected for 30 out of 32 sites; right: the "detection rate" for the algorithms used for the EIP temperature means (Böhm et al, 2008). 
ausgerichtet. Bei den LSS-Stationen kamen Abweichungen von der Nordrichtung von $-40^{\circ}$ bis $+30^{\circ}$ vor. Meistens zu dem Zeitpunkt, als ein Wetterdienst die LSS-Station in sein Messnetzmanagement aufnahm, wurden genormte, damals aus Blech bestehende so genannte Fensterhütten zur Abschirmung verwendet, wodurch ein wesentlicher Schritt zur Minimierung des Strahlungsfehlers getan war.

Gleichzeitig erfolgte auch eine gewisse (aber nicht vollständige) Normierung der Beobachtungszeiten. Für die EIP konnte diese im Hinblick auf den EIP-Bias eminent wichtige Information für 1331 von den insgesamt vorhandenen 1735 EIP-Jahren aus den vorhandenen historischen Beschreibungen extrahiert werden. Das bedeutet eine „detection-rate“ von 77\% - eine ganz ansehnliche Quote für so lange zurückliegende Zeiten, für manche Einzelreihen aber trotzdem ein knockout-Kriterium für eine direkte Bias-Korrektur. Für diese Reihen mit ungenügender Dokumentation der Stationsgeschichte (in Böhm et al., 2008 im Detail angeführt) wurde eine nachträgliche Korrektur angebracht, die wie eine entsprechende Homogenisierung von Breaks mit Hilfe von relativen Homogenitätstests durchgeführt wurde (Peterson et al., 1998, Aguilar et al., 2003, Auer et al., 2007).

Zunächst gar nicht und auch später nur sehr schleppend wurde von den Fensterhütten zu der modernen Aufstellung in Gartenhütten (offizielle WMO-Bezeichnung „Stephenson screen") gewechselt. Diese Entwicklung (Abb. 3) dauerte in manchen Fällen bis zur Mitte des 20. Jahrhunderts und ist in einigen Fällen noch heute nicht abgeschlossen. Letzteres gilt besonders für Italien, dessen offizieller „Wetterdienst“ und WMO-Vertreter die „Aeronautica Militare“ ist, die kaum irgendwelche Intentionen in Richtung Qualitäts-Klimadienst zeigt. Glücklicherweise hat von den drei, die EIP charakterisierenden Faktoren (Abschirmung, Messtermine, Höhe über Grund), letzterer die geringste Auswirkung, da sich die wesentlichen vertikalen Temperaturunterschiede bereits unterhalb der modernen Aufstellungshöhe (ca. 2m) abspielen (Geiger et al., 2005).

Nachdem also, wie beschrieben, die Metadatensituation der frühen instrumentellen Periode recht befriedigend abgeklärt werden konnte, waren die Voraussetzungen für eine EIP-Korrektur gegeben. Die Notwendigkeit einer derartigen Korrektur, nachdem ja der

Abb. 3: Die zeitliche Entwicklung der Aufstellungshöhe der Thermometer an 98 HISTALP Stationen, für die diese Metadaten eruiert werden konnten.

Fig. 3: The level above ground for 98 HISTALP stations, where this parameter could be obtained for the past years.

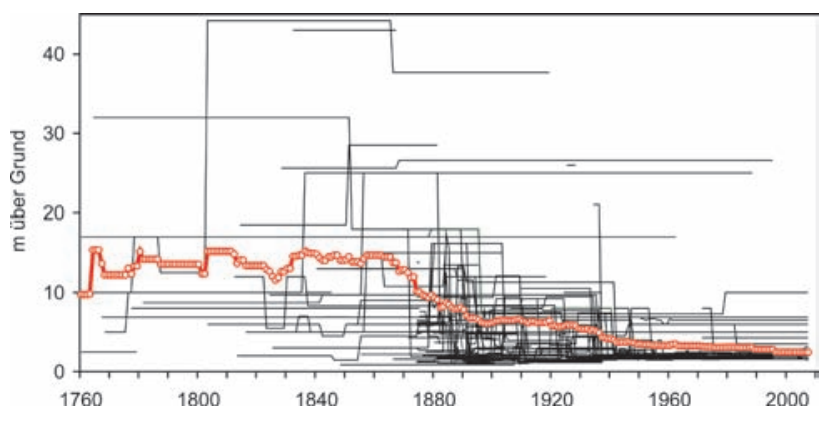




\section{Klimawandel in Österreich}

gesamte Datensatz in der bei Auer et al. (2007) beschriebenen ,state of the art“ Prozedur bereits homogenisiert worden ist, erwächst aus einem typischen Problem, an dem alle entsprechenden mathematisch-statistischen Methoden leiden, die ja in ihrem Kern auf demselben Grundprinzip beruhen: Man berechnet Differenz- oder Quotientenreihen hoch-korrelierter Nachbarreihen, testet sie auf abrupte Sprünge, die statistisch signifikant sind, und eliminiert diese unter der Annahme der angenommenen zeitlichen Stationarität der Differenzen (Quotienten). Da üblicherweise alle Reihen irgendwann nicht-klimatologische Sprünge haben, gilt es noch das Problem der Zuordnung zu lösen, welche der Reihen die infizierten und welche die Referenzreihen für ein betrachtetes Subintervall mit einer Sprungstelle sind. Das kann man mit statistischen Wahrscheinlichkeitsüberlegungen abklären, indem man in regionalen Subgruppen von typischerweise 10 Reihen alle Reihen mit allen anderen testet. Die sich ergebende Entscheidungsmatrix (typischerweise 10*10) liefert dann recht eindeutige Ergebnisse, wenn die Sprünge nicht alle zur selben (ähnlichen) Zeit erfolgen.

Gerade das war jedoch in den 250 Jahren der instrumentellen Periode im Alpenraum bisher viermal der Fall. Das jüngste Beispiel ereignet sich gerade jetzt: Im gesamten Gebiet und darüber hinaus wurden in den 1980er und 1990er Jahren die Klimamessnetze automatisiert. Ob diese simultane Umstellung zu systematischen Inhomogenitäten führt bzw. geführt hat, lässt sich erst in naher Zukunft analysieren, wenn sich die Situation wieder „beruhigt“ hat. Man kann nur hoffen, dass die Wetterdienste sich an ihre eigenen Qualitätskriterien halten und genügend Vergleichsregistrierungen vorliegen werden, denn auf die mathematisch-statistische Art allein wird die sicher notwendige Homogenisierung nicht unproblematisch werden.

Am 1. Jänner 1971 ereignete sich eine gut dokumentierte Inhomogenität in der Schweiz und in Österreich durch den Beschluss, den Abendbeobachtungstermin im Klimadienst um 2 Stunden vorzuverlegen, samt einer Änderung der entsprechenden Mittelwertsformeln. Angeblich erfolgte diese unglückliche Änderung notgedrungen, da die Beobachtungsqualität durch das damals stark aufkommende Fernsehen immer mehr nachgelassen hatte. Im Nachhinein gesehen war dieser Beschluss der beiden Wetterdienste nicht sehr sinnvoll, da ja nicht lange danach dieses Problem durch die in den 1980er Jahren einsetzende Automatisierung der Messnetze obsolet geworden wäre. Wenigstens ließ sich post festum diese Inhomogenität mit Hilfe eben dieser Stundenwertsdaten der automatischen Messnetze genau definieren und beseitigen.

Die dritte Periode mit vielen Umstellungen und auch Lücken in einem kurzen Zeitraum waren die unseligen 1940er Jahre. Der zweite Weltkrieg verursachte neben einem allgemeinen Verfall des Messnetzes, Beobachtermangel, Stationseinstellungen auch eine durchaus interessante systematische „De-urbanisierung“ vor allem der langen historischen Klimareihen. Diese waren ja traditionell eher in den Stadtzentren angesiedelt gewesen und 
wurden nun in größerer Zahl auf die vielen neu angelegten zunächst militärischen, später zivilen Flugfelder und Flughäfen verlegt. Diese systematische Inhomogenität konnte für den Alpenraum jedoch korrigiert werden, da noch genug unbeeinflusste Zeitreihen vorlagen (siehe Diskussion dazu in Böhm et al., 2001). Die Korrektur führte zu einer Absenkung des ursprünglichen Temperaturniveaus vor den 1940er Jahren in Relation zu dem danach um einige Zehntel Grad.

Die vierte Periode schließlich mit Verdacht auf eine systematische großflächige Inhomogenität ist der beschriebene Übergang von der EI-Messphilosophie auf die darauf folgende der regulären Wetterdienste mit dem Schwerpunkt in den 1860er Jahren. Neben den bereits diskutierten physikalischen Gründen gab es auch entsprechende Hinweise von indirekten Klimadaten aus der Region (Frank et al., 2007, Hiebl et al., 2006, Büntgen et al., 2005) und von (wenigen) entsprechenden Arbeiten aus dem instrumentellen Bereich in anderen
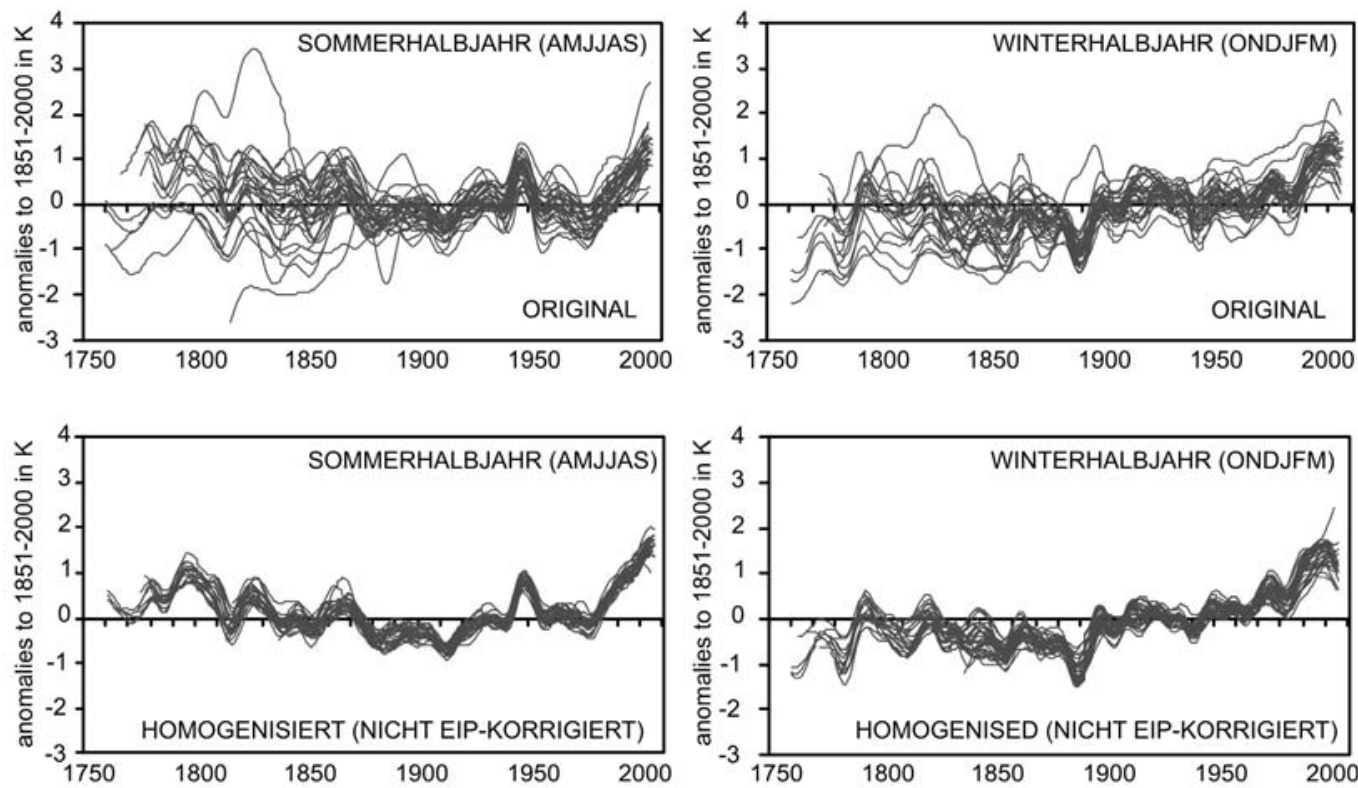

Abb. 4: Die Temperaturzeitreihen der 32 LSS-Stationen im Großraum Alpen von 1760 bis 2007 (2007/08). Oben im Originalzustand (nicht homogenisiert, nur Outlier-bereinigt), unten nach der Homogenisierung in der Version 2007 (Auer et al., 2007). Zur besseren Vergleichbarkeit wurden die Reihen geglättet (21-jähriger Gauss'scher Tiefpassfilter) und alle als Anomalien zur gemeinsamen Bezugsperiode 1851-2000 dargestellt.

Fig. 4: Temperature time series of the 32 LSS-stations in greater area of the Alps 1760-2007 (2007/08). Upper panels show origininal data (not homogenized, outliers removed); the lower graphs show homogenized data (Auer et al., 2007). The left panels refer to the summer months (AMJJAS), the right panels to winter (ONDJFM). To improve comparability the series have been smoothed by a 21 -year Gaussian low-pass filter. All series are plotted as anomalies compared to the reference period $1851-2000$. 


\section{Klimawandel in Österreich}

Regionen (Moberg et al., 1997 und 2003). Als Ausgangspunkt lag für den Großraum Alpen die Version 2007 des HISTALP Datensatzes vor. Wie der Vergleich der oberen beiden Zeitreihen der Abb. 4 (die Originaldaten der 32 LSS-Temperaturreihen für das Sommer- und das Winterhalbjahr) mit den unteren beiden (die homogenisierten der Version 2007) zeigt, ist die Homogenisierung, die zur Version HISTALP-2007 geführt hat, ganz gut gelungen. Zwar ist die visuell ins Auge springende „Beruhigung“ des doch recht „lebhaften Patchworks“ der Originalreihen nicht das einzige Kriterium, aber ein wichtiges, speziell wenn die etwa in Auer et al. (2007) diskutierte hohe räumliche Kovarianz von jahreszeitlich gemittelten Temperaturreihen in Rechnung gestellt wird, die die starke Streuung der Originalreihen als Artefakt von Inhomogenitäten und nicht als echtes Klimasignal einstufen lassen. Die Graphik links unten in Abb.4 weist auch auf das potentielle EI-Problem hin. Es ist der generell recht ausgeprägte negative Trend von der verdächtig warmen Periode um 1800 zur kalten Phase zu Ende des 19. und am Beginn des 20. Jahrhunderts. Dem stehen die kühleren Proxy-EIP-Sommer gegenüber, wie auch die Klimaantriebe (Bard et al, 2000, Lean, 2000, Robertson et al., 2001) und die vorhandenen historischen Modelläufe (Zorita et al., 2004), welche alle auf ein niedrigeres Sommertemperaturniveau hindeuten. Zur Problemlösung konnten diese jedoch aus den erwähnten Gründen nicht verwendet werden - dazu wollten wir streng innerhalb der instrumentellen Domäne bleiben.

\section{Die Lösung mit Hilfe der Parallelregistrierung in Kremsmünster}

Es galt also, quantitative Information über den EIP-Bias zu erlangen, daraus monatliche Korrekturgrößen abzuleiten und in den in Abb. 2 gezeigten Zeitspannen anzubringen. Eine Literatur- Recherche und auch persönliche Anfragen ergaben, dass es die dazu notwendigen direkten Messdaten aus Parallelregistrierungen an erhaltenen historischen Messplätzen und modernen Aufstellungen in der Region und auch anderswo einzig an einem Ort gibt, aber dafür dort bereits in der Form eines mehrjährigen Datensatzes in hoher zeitlicher Auflösung. Es handelt sich um die Klima-Langzeitstation des Stifts Kremsmünster (Oberösterreich), wo seit der Automatisierung in den 1980er Jahren zusätzlich zu den manuellen Messungen auch automatische Registrierungen vorliegen, sowohl an der komplett erhaltenen historischen Installation, als auch an der modernen Gartenaufstellung (Abb. 5). Für die Jahre 1995-2002 sind 8 lückenlose und geprüfte Stundenwertsdatensätze für beide Standorte vorhanden, die die Basis für die weitere Vorgangsweise bildeten. Abb. 6 zeigt die mittleren Temperaturdifferenzen historisch minus modern für die 12 Monate des Jahres. Aus diesen können somit für alle ähnlich orientierten (nach NNE) frühen Stationen für beliebige Mittelwertsformeln die EIP-Korrekturen berechnet werden. 

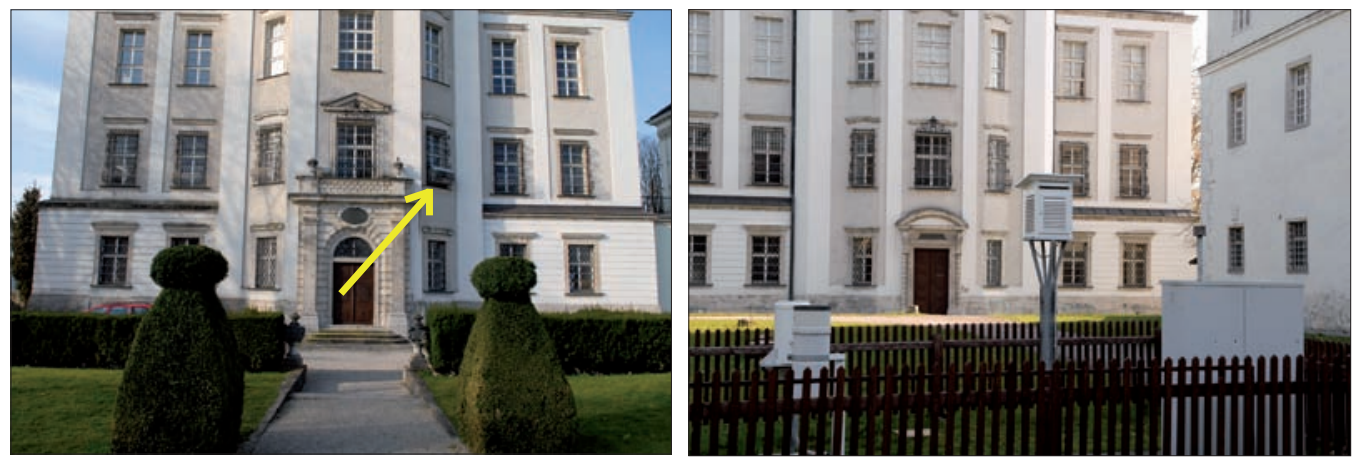

Abb. 5: Die bistorische (links) und die moderne (rechts) Aufstellung der Temperaturmessgeräte an der Klimastation Kremsmünster (Fotos: Böhm)

Fig. 5: Historical (left) and modern (right) placement of temperature recorders at Kremsmünster (Photos: Böhm)

Natürlich waren nicht alle dieser historischen Stationen N-30-E orientiert wie Kremsmünster, weshalb die folgende einfache Methode notwendig war, um andere Orientierungen und damit andere Tagesgänge der Temperaturdifferenzen - zu simulieren. In Ermangelung an Messdaten außer denen von Kremsmünster und in Ermangelung an echten Informationen über die Wandstärken der Gebäude, die Baumaterialien und den Oberflächenanstrich, bestand die einzige Möglichkeit in der Spiegelung der Tagesgänge von Abb. 6 an der 12-Uhr Linie. Dieser, bei der gegebenen Daten- und Metadatenlage bestmögliche Ansatz, erlaubt es, analoge Tagesgänge der Differenzen historisch minus modern auch für NNW-orientierte Aufstellungen zu simulieren und als Mittel aus NNE und NNW schließlich diejenigen für reine Nord-Orientierung zu berechnen. Eine detailliere Begründung samt Diskussion der Methode ist in Böhm (2008) enthalten. Abb. 7 zeigt die simulierten mittleren Temperaturdifferenzen (jeweils für die ersten 6 Monate des Jahres) für die NNW- und die N-Orientierung.

Nun konnten Standort für Standort und Subintervall für Subintervall für die jeweils bekannten Mittelungsformeln die monatlichen EIP-Korrekturen berechnet und angebracht werden. Abb. 8 zeigt alle einzelnen Jahresgänge der EIP-Korrekturen und verdeutlicht zum einen die Notwendigkeit, jede Station und jedes Subintervall getrennt zu behandeln, da die Streuung doch recht groß ist. Zum anderen erkennt man trotzdem die erwartete Systematik mit den stärksten negativen Korrekturen von durchschnittlich $0.5^{\circ} \mathrm{C}$ im Juni und den leicht positiven im Februar und März. Da ja, wie das rechte Diagramm der Abb. 2 gezeigt hat, nicht alle historischen Metadaten eruiert werden konnten, verblieben vorerst noch einige EIPSubintervalle von wenigen Stationen unkorrigiert. Sie wurden in der Folgen an die korrigierte Mehrheit angepasst, wobei das in unserer Arbeitsgruppe standardmäßig eingesetzte Homogenisierungssystem HOCLIS zur Verwendung kam (Auer et al., 2007). 
Klimawandel in Österreich
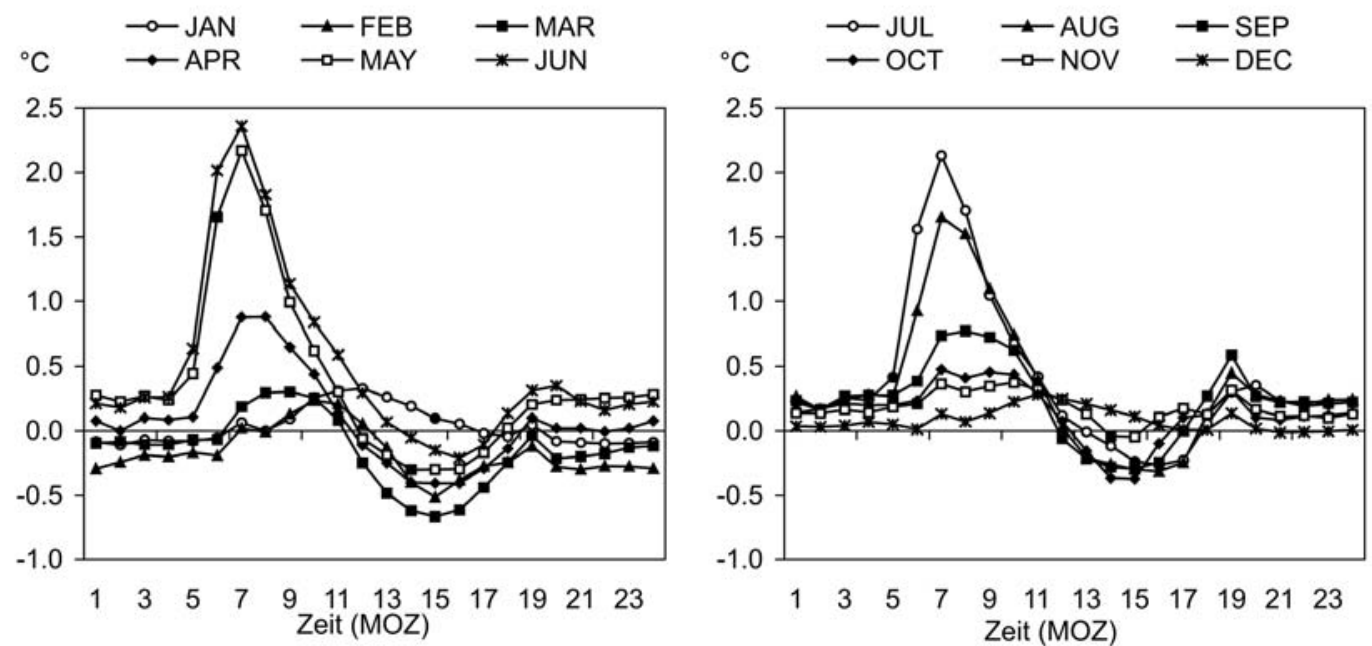

Abb. 6: Mittlere Differenzen historisch minus modern der beiden Temperatur-Messstandorte in Kremsmünster für Jan-Jun (links) und Jul-Dę (rechts). Basis: lückenlose Stundenwertsdaten 1995-2002

Fig. 6: Mean temperature difference between historic and modern temperature recordings in Kremsmünster for January-June (left) and July-Dezember (right). Based on complete hourly time series 1995-2002
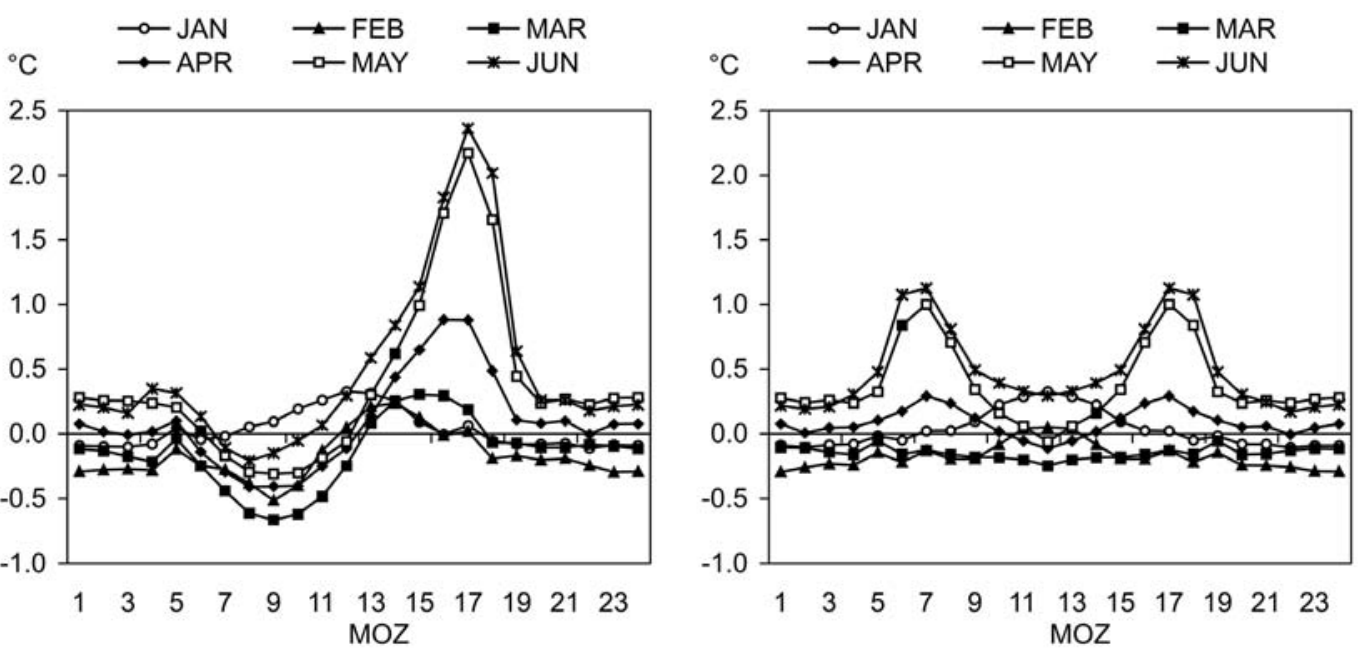

Abb. 7: Mittlere Differenzen bistorisch minus modern für NNW-orientierte (links) und N-orientierte (rechts) Temperaturmessstandorte simuliert aus den Messdaten Kremsmünster aus Abb.6. Gezeigt sind nur die ersten sechs Monate des Jahres.

Fig.: 7: Mean temperature difference between historic minus modern temperature recordings for NNW-orientated (left) and N-orientated (right) temerature recordings. Simulation is based on the data from Kremsmünster from Fig. 6. Only the first six months of the year are shown. 
Abb. 8: EIP-Korrekturen, die zur Beseitigung des EIPBias an die frühen instrumentellen Temperaturreiben (siehe Abb.2) angebracht wurden. Dünn: die einzelnen Korrekturen, rot: das Mittel aller Korrekturen.

Fig. 8: EIP-corrections used to remove the EIPbias from early instrumental temerature series (see Fig. 2). Thin lines represent individual corrections; red: the mean of all corrections.

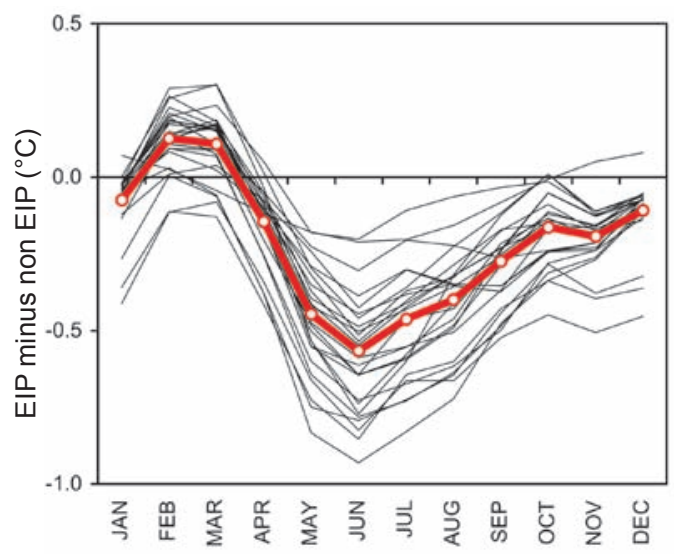

Alles in allem kann der hier skizzierte und in Abb. 9 dargestellte Weg von den Originaldaten der Version 2007 zu der EIP-bias korrigierten Fassung 2008 des HISTALP LSS-Temperaturdatensatzes als erfolgreiches Unterfangen angesehen werden, um der „Wahrheit“ des Temperaturklimas der frühen instrumentellen Phase im Alpenraum wieder einen Schritt näher zu kommen. Es stehen nun 32 EIP-korrigierte Zeitreihen zur Verfügung, die zu den in globaler Abdeckung vorhandenen 150 Jahren instrumentellen Temperaturklimas (Jones and Moberg, 2003, Brohan et al., 2006) weitere 100 Jahre frühen instrumentellen Temperaturklimas hinzufügt, denen nun eine höhere Glaubwürdigkeit zugebilligt werden muss als
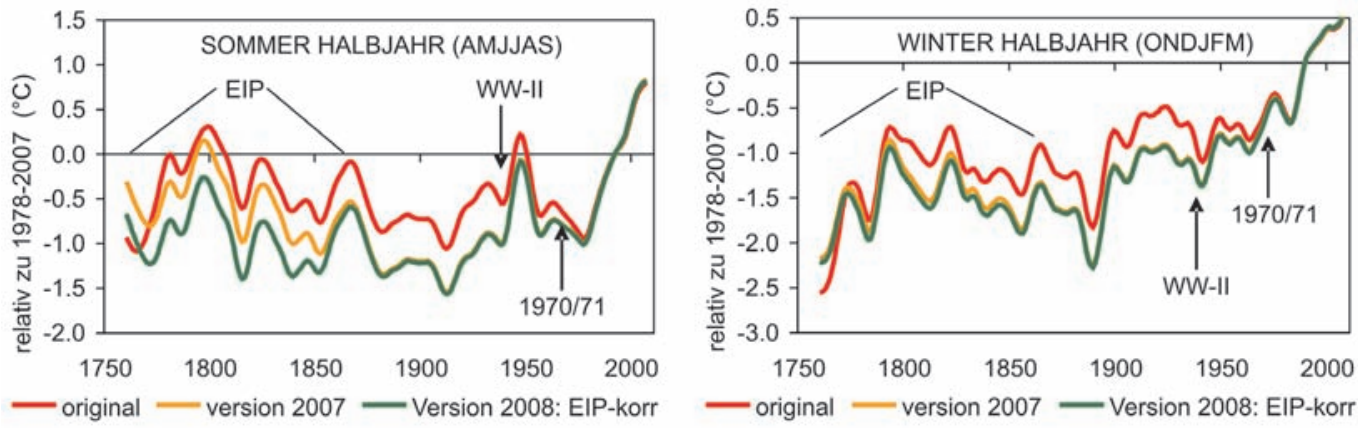

Abb. 9: Der Weg von den nicht homogenisierten (rot) über die homogenisierten Version-2007 (orange) jahreszeitlichen mittleren Temperaturreihen im Großraum Alpen zu der aktuellen, EIP-bias-korrigierten Fassung 2008 (grün). Alle Zeitreiben 1760-2007 (2007/08) sind 21-jährig Tiefpass-gefiltert und als Abweichungen vom aktuellen Mittel 1978-2007 dargestellt. Die Pfeile markieren zwei besprochene systematischen Inhomogenitäten. Datenbasis: die 32 LSS Monatsreiben der HISTALP Datenbank der ZAMG

Fig. 9: From non homogenized (red) to homogenized temperature series (verison-2007, orange) in the greater Alpine area compared to the updated EIP-bias correction (version-2008, green). All time series, 1760-2007 (2007/08) are filtered with a 21-year low pass filter and deviations from the current mean (1978-2007) are shown. Arrows indicate two systematic inhomogenities mentioned in the text. Data based on 32 LSS monthly time series of the HISTALP data base at the ZAMG 


\section{Klimawandel in Österreich}

der bisher vorhandenen Version 2007. Man erkennt in der Abbildung 9 zunächst nochmals die unbedingte Notwendigkeit des Homogenisierens. Das oft gehörte Argument, dass bei Verwendung genügend vieler Einzelreihen die Inhomogenitäten in der Mittelreihe als „random effect“ verschwinden würden, ist nicht zutreffend. Es gibt sehr wohl systematische Inhomogenitäten, die auch größere Gebiete betreffen. In Abb. 9 sind zwei dieser bereits eingangs besprochenen Breaks mit Pfeilen markiert, die beide in Summe die Periode vor dem 2. Weltkrieg in den Originaldaten systematisch etwa um $0.5^{\circ} \mathrm{C}$ zu warm erscheinen lassen (rot gegenüber gelb). Der Vergleich der gelben Kurven (Version 2007) mit den grünen der EIP-korrigierten Version 2008 hingegen zeigt die erwartete und diskutierte quantitative Wirkung der bias Korrektur in der frühen instrumentellen Periode. Im Sommerhalbjahr ergab das in Summe noch einmal eine Abkühlung gegenüber den Originaldaten von etwa $0.4^{\circ} \mathrm{C}$, im Winterhalbjahr lediglich eine um weniger als $0.1^{\circ} \mathrm{C}$ - als Ergebnis der gegenläufigen Korrekturen im Februar und März gegenüber denen von Oktober bis Jänner.

Die neuen regional gemittelten langjährigen Temperaturverläufe der bis 1760 zurückreichenden und bis März 2008 aktualisierten bias-korrigierten HISTALP-2008 LSS Reihen sind in der Abb. 10 dargestellt. Sie werden nun wohl, abgesehen von künftigen Updates, für längere Zeit das Optimum an instrumenteller Information im Großraum Alpen darstellen. Anders als in der bereits seit einiger Zeit angezweifelten (Hiebl, 2006, Fank et al, 2007) Version 2007 kommt nun auch der frühen instrumentellen Phase eine höhere Glaubwürdig-
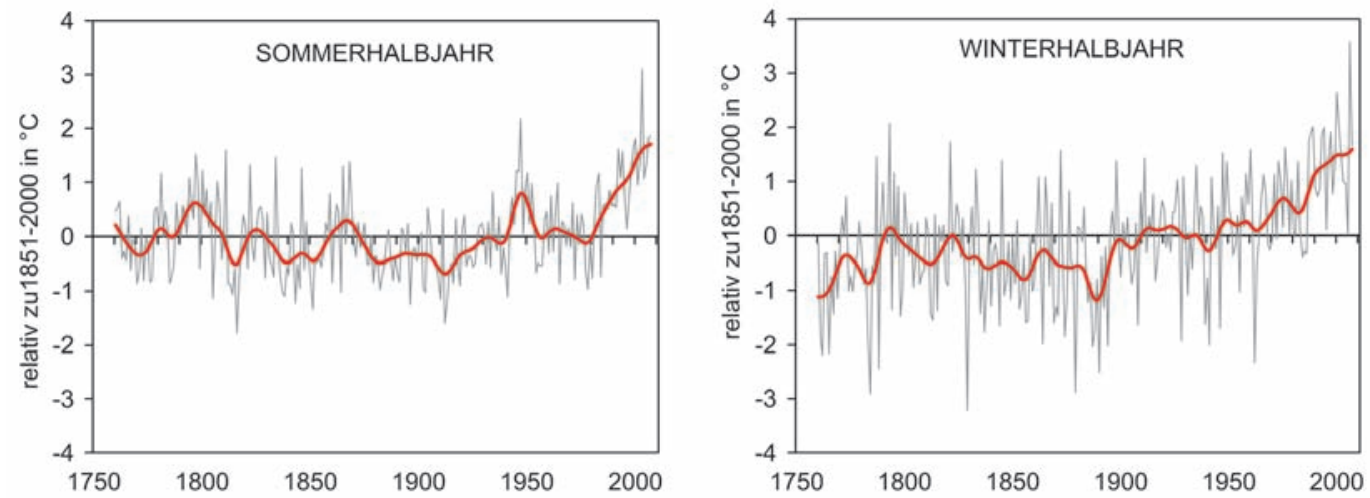

Abb. 10: Die jahreszeitlichen mittleren Temperaturkurven 1760-2007 (2007/08) des Großraums Alpen in der Version 2008. Links: Sommerhalbjahr, rechts Winterhalbjabr. Einzeljabre (grau) und 21 jährig geglättet (rot). Dargestellt sind Abweichungen zum Mittel 1851-2000. Datenbasis: die 32 LSS Monatsreihen der HISTALP Datenbank der ZAMG

Fig. 10: Annual mean temperatures in the greater Alpine area, 1760-2007 (2007/08, version-2008). Left: summer; right: winter half-year. Annual means (grey) and 21 year low-pass filtered data (red) are shown as deviation from the average 1851-2000. Data based on 32 LSS monthly series of the HISTALP database at the ZAMG 
keit zu. Eine ausführlichere Beschreibung wird demnächst im Sonderband des EU-Millenniums Projekts der Zeitschrift Climatic Change erscheinen (Böhm et al., 2008).

\section{Problem 2: Homogenisierung von Tages- und Subtagesdaten}

Ein zweites Problem auf dem Gebiet der Homogenisierung ist das von Tages- und Subtagesdaten. Gerade diese stehen ja zurzeit sehr im Vordergrund des Interesses, da ja „der Klimawandel" zu einer Intensivierung der extremen Ausformungen des Klimas (Wetters) führen soll. Gerade diese, meist kleinräumigen und kurzzeitigen Phänomene, entziehen sich jedoch noch weitgehend einer Homogenisierung und die wissenschaftlich saubere Behandlung vieler Fragestellungen über Trends der Extremwerte ist dadurch stark eingeschränkt. Warum das so ist und welche Strategien dieses Defizit reduzieren sollen, sei hier als Ausblick auf die entsprechenden Pläne der ,instrumentellen Klimarekonstrukteure“ besprochen und die gegenwärtigen Arbeiten daran skizziert.

Wohl die größte Diskrepanz zwischen harten wissenschaftlichen Fakten und deren Wahrnehmung durch die Öffentlichkeit besteht auf dem Gebiet der extremen Ausformungen des Klimas. Unter dem allgemein akzeptierten Schlagwort „das Klima wird immer verrückter" hat sich ein Dogma festgesetzt, das gerade bei den weichsten Fakten ansetzt, die die klimatologische Wissenschaft derzeit (noch?) zu bieten hat. Weiche Faktenlage bei den Extremwerten herrscht sowohl bei der Klimarekonstruktion der Vergangenheit als auch bei der Klimamodellierung (durch Atmosphäre-Ozean-gekoppelte globale Zirkulationsmodelle, AOGCM), in beiden Fällen begründet durch eine Tatsache, die im Kapitel 8.5.4 des Working Group 1 Reports von IPCC-2007 (Model Simulation of Extremes) so beschrieben ist:

Because most AOGCMs have coarse resolution and large-scale systematic errors, and extreme events tend to be short lived and have smaller spatial scales, it is somewhat surprising how well the models simulate the statistics of extreme events in the current climate, including the trends during the $20^{\text {th }}$ century. This is especially true for the temperature extremes, but intensity, frequency and distribution of extreme precipitation are less well simulated.

Ich habe es absichtlich beim englischen Originaltext belassen, erstens um dem Vorwurf der Manipulation zu entgehen, zweitens aber, weil ich ein Fan der Zwischentöne bin, die diese Sprache bietet. Dieses „somewhat surprising“ und das „less well“ sind schon kleine Meisterstücke. Im Original des Textes, bevor der mühsame Prozess des „,peer review“ durchgefochten werden musste, war noch etwas direkter von ,,serious deficiencies in the simulation of precipitation“ die Rede, und zwar ,both in the intensity and the distribution of precipitation".

Im Hinblick auf die Analyse eventueller Trends von Extremwerten in der Vergangenheit kommen noch zwei zusätzliche Herausforderungen dazu. Zum einen erfordern die 


\section{Klimawandel in Österreich}

Gesetze der Statistik lange und räumlich dichte Zeitreihen, um zu signifikanten Ergebnissen zu kommen, gerade wenn die sehr seltenen starken Ausreißer das Ziel der Analyse sind. Und genau diese langen Zeitreihen erfordern als conditio sine qua non eine sorgfältige Homogenisierung. Wie wir zeigen konnten (Auer et al., 2007) erfährt eine durchschnittliche Klimazeitreihe im Durchschnitt alle 20 bis 30 Jahre eine Diskontinuität (Verlegung, Instrumentenoder Technologiewechsel, Änderung der Umgebung und zahlreiche andere Gründe), deren Stärke das eigentliche Klimasignal erreichen oder auch übertreffen kann. Und gerade für die zur Analyse von „short lived“ Extremwerten besonders notwendigen Klimazeitreihen von Tageswerten (oder noch kürzerer Zeitspannen) zeigen eine zweite Besonderheit der entsprechenden Feldverteilungen: Sie dekorrelieren räumlich viel stärker als längerfristige Mittelwerte oder Summen. Abb. 11 zeigt das exemplarisch für die Tages- bis Jahreswerte von Temperatur und Niederschlag, wobei hier noch die zusätzliche besondere „Empfindlichkeit" des Klimaelements Niederschlag hervortritt.

Da zur Homogenisierung der Zeitreihen (=Anpassung früherer Zustände an den aktuellen Zustand einer Messreihe) hoch-korrelierte Zeitreihen benachbarter Referenzreihen benötigt werden, zeigt die Abb. 6 den Hauptgrund für die leider derzeit noch gegebene Tatsache, dass es homogenisierte Klimazeitreihen auf Tagesbasis kaum gibt. Das gilt sowohl international als auch für den Alpenraum oder Österreich. Für einige Subregionen Österreichs arbeitet unsere Gruppe gerade an der Erstellung räumlich dichter Tagesreihen für das 20. Jahrhundert und eine internationale COST-Aktion („HOME“ siehe Anhang) hat u.a. das Problem der Homogenisierung von Tagesreihen zum Gegenstand.

Spiegelbildlich dieselben Probleme zeigen sich bei der Trendanalyse der Extremwerte. Deren große räumliche und zeitliche Variabilität rückt lokale Ergebnisse in den Vordergrund und verdeckt den Überblick auf die Gesamtsituation. Naturgemäß eröffnet das in der öffentlichen, aber auch in der wissenschaftlichen Debatte, ein weites Feld entbehrlichen

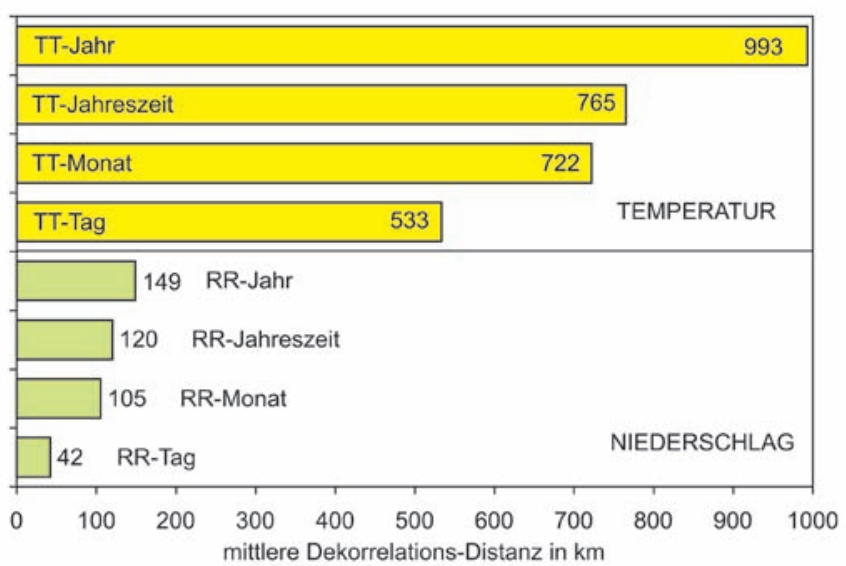

Abb.11: Räumliche Dekorrelation auf 50\% gemeinsame Varianz im Europäischen Klimamessnetz, von Jahres, Jahreszeiten-, Monats- und Tageswerten von Temperatur (T) und Niederschlag (R). Quelle: Scheifinger et al., 2003 (interner Projektsbericht für Projekt ALP-IMP).

Fig. 11: Spatial decorrelations down to $50 \%$ common variance in the European climate measurement network of yearly, monthly, and dayly measurements of temperature (T) and precipitation (R). Scheifinger et al., 2003; ALP-IMP internal project report. 
Streits, widersprüchlicher Ergebnisse und das Eindringen weltanschaulicher Argumentation in ein wissenschaftlich noch nicht befriedigend aufgearbeitetes Thema. Immer kann in den zweifellos real vorhandenen, aber noch nicht flächen- und zeitabdeckend analysierten Mustern der Extremwert-Verteilungen, gerade das Ereignis oder Ergebnis herausgesucht werden, das am besten die jeweilige Position untermauert - „picking“ heißt der dafür existierende Fachausdruck. Um nicht in genau diese Falle zu tappen, werden in der Folge einige vorläufige Ergebnisse vorgestellt, wobei das bisher gesagte die notwendige Einschränkung im Hinblick auf Verallgemeinerung klargestellt haben sollte.

Ein interessantes vorläufiges Ergebnis, das unsere laufenden diesbezüglichen Projekte erbracht haben bzw. gerade erbringen (Foralps, A Tale of two valleys), ist vor allem beim Niederschlag der überraschend enge Zusammenhang zwischen Trends von Extremwertindizes auf Tagesbasis und den entsprechenden Trends der monatlichen Mittelwerte bis hin zu Jahresmitteln. Abbildung 12 bringt ein Beispiel für extremen Tagesabfluss und Niederschlag im großen Donaueinzugsgebiet des Pegels Nussdorf (Wien), Abbildung 13 einen Vergleich von Niederschlagsjahressummen und Tageswertsindizes einer Klimareihe in den Hohen Tauern. Es scheint somit, zumindest für den Großraum Alpen, nicht so zu sein, dass Trends der Extremwerte in ganz andere Richtungen gehen könnten als die der Mittelwerte. Es ist also nicht unbedingt zu erwarten, dass zum Beispiel in Gegenden mit generell fallendem Niederschlagstrend (wie etwa im Südosten des Alpenbogens) ein Anstieg der Starkregen zu erwarten ist oder, im umgekehrten Fall, in Regionen mit Niederschlagszunahme (wie etwa im Nordwesten des Alpenbogens), die Trockenperioden häufiger werden.

Dieser Befund gilt auch für die Temperatur: Mit generellem Erwärmungstrend steigen die Extremwerte, die sich auf Hitze beziehen, und im gleichem Maß ist ein Rückgang der Kälteindizes zu beobachten. Beides nicht notwendigerweise in linearer Form. Speziell wenn Schwellwertsüber- oder -unterschreitungen analysiert werden, sind nichtlineare Zusammenhänge die Regel.

Der vorläufige Befund der Ähnlichkeit der Trends von auf Tageswerten beruhenden Extremwerten und denen der Mittel- oder Summenwerte, lässt auch einem weiteren vorläufigen Ergebnis erhöhte Bedeutung zukommen, das eine noch unveröffentlichte Untersuchung an den längsten HISTALP-Monats-, Jahreszeiten- und Jahresreihen von Luftdruck, Temperatur und Niederschlag erbracht hat. Es wurden die längsten Zeitreihen der Region (jeweils 20 bis 30 Reihen), die alle in die frühe instrumentelle Periode zurückreichen (vor 1840), nach einem hier nur kurz skizzierbaren Verfahren auf langfristige Veränderungen der Variabilität untersucht.

Das Verfahren wurde auf alle Reihen getrennt angewendet, um die statistisch zu erwartende Erhöhung der Varianz mit abnehmender Reihenanzahl zu vermeiden. Bei der Jahres- 

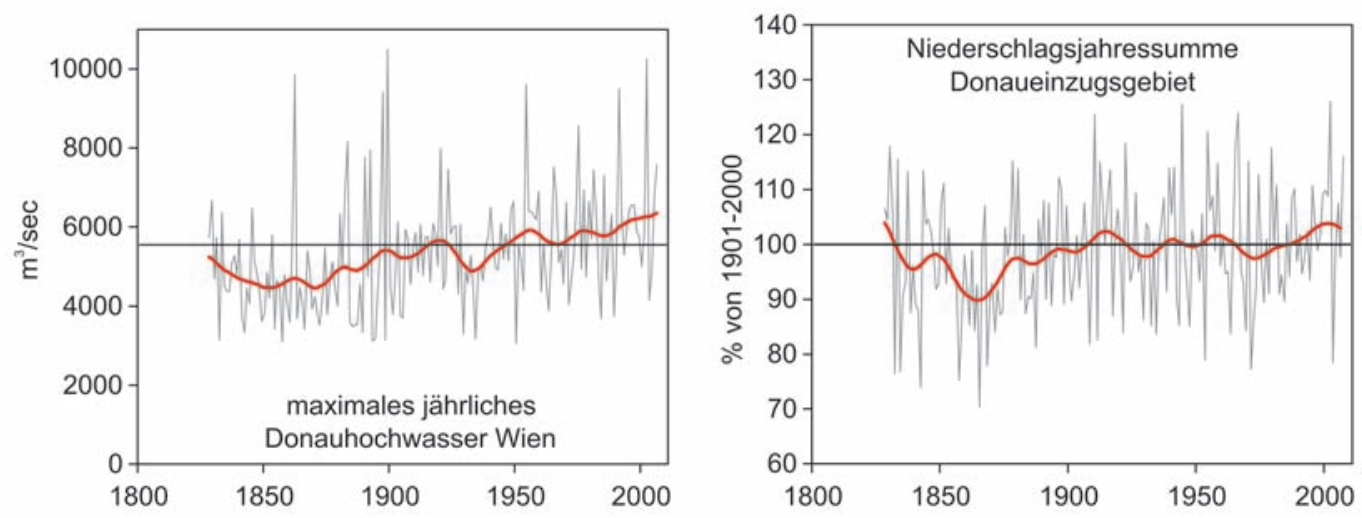

Abb.12: Links: Zeitreibe der jährlich größten Durchflussmengen der Donau bei Wien, 1828-2006, Quelle: Godina R, Hydrographisches Zentralbüro, Wien, 2008, persönliche Mitteilung; Rechts: Flächenmittel der Jabressummen des Niederschlages im Einzugsgebiet der Donau oberbalb Wiens (Auer et al., 2007). Alle Reihen für Einzeljahre (grau) und 30 jährig (rot) geglättet (Gauss'scher Tiefpassfilter)

Fig 12: Left: Time series of the Danube maximum water discharge at Vienna, 1828-2006. From: Godina R 2008., Hdrographic Center, Vienna, pers. communication; right: area means of the annual precipitation in the catchment of the Danube before Vienna (Auer et al., 2007). Data for individual years (grey) and 30yr filtered (red, Gaussian low pass filter) are shown.

zeiten- und Jahresanalyse wurden zunächst die langfristigen Trends selbst durch Hochpassfilterung eliminiert (Filterweite 31 Jahre) und dann in übergreifenden Zeitfenstern von 30 Jahren Quantile (von 1\% bis 99\%) berechnet. Dadurch konnten mit den Interquantilbereichen verteilungsfreie Zeitreihen der Klimavariabilität erzeugt werden. Analysiert wurden jeweils die 80-, 90- und 98\% Interquantilbereiche. Für (durch Normalisierung) Jahresgangbereinigte sequentielle Monatsreihen wurde das analoge Verfahren auf der Basis von 10jährigen (=120 monatlichen) Zeitfenstern durchgeführt.

Bei Luftdruck und Temperatur ergaben sich regional sehr ähnliche Verläufe der Interquantil-Zeitreihen, beim Niederschlag reagierte der mediterrane, der atlantische und der kontinentale Bereich des Großraums Alpen regional und jahreszeitlich unterschiedlich. Generell wurde sowohl in den letzten 200 Jahren, als auch in den letzten 50 Jahren keine Steigerung der Klimavariabilität beobachtet, wie sie von den Medien oft als bewiesen vorausgesetzt wird. Speziell bei der Temperatur sind die Trends der Variabilität (= der „Verrücktheit des Klimas") in allen Jahreszeiten und in allen Subregionen rückläufig. Abbildung 14 illustriert das am Beispiel der sequentiellen Monatsreihen der Lufttemperatur, gemittelt über alle 24 Langzeitreihen. Beim Niederschlag liegen jahreszeitlich und regional unterschiedliche Trends der Variabilität vor, wobei die markantesten Zunahmen der Variabilität typischerweise dort beobachtbar sind, wo auch die Trends der Niederschlagsmengen zunehmen, zum Beispiel im Herbst und Winter der Subregion nordwestlich des Alpenbogens. Tabelle 

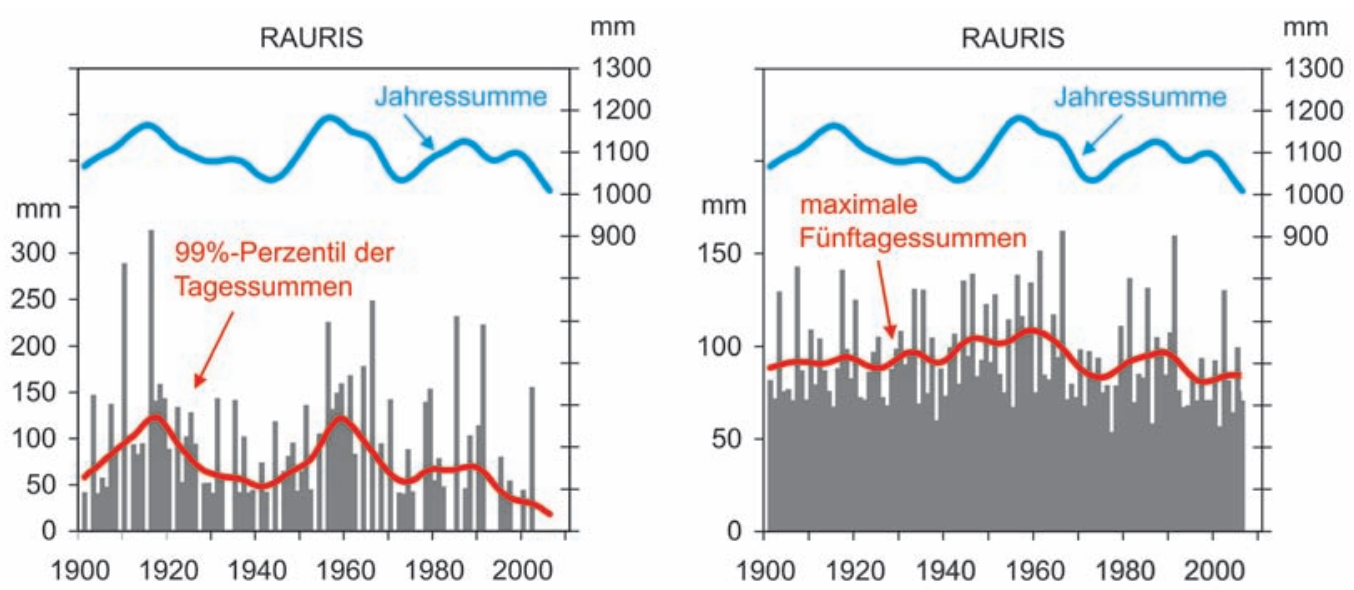

Abb.13: Ähnlichkeit zwischen der gesamten Jahressumme des Niederschlages (blau, 21-jährig geglätteten Linien) und von zwei Niederschlags-Extremwertindizes für den inneralpinen Ort Rauris (Einzeljahre und 21-jäbrig geglättet, unten). Links: Summe der Tagesniederschläge, die in der Klimanormalperiode 1961-90 nur noch von 1\% aller Fälle übertroffen werden (= 99\%-Perzentil); rechts: maximale Fünftagessummen eines Jahres (Auer et al., 2008a, b).

Fig. 13: Smilarity between the annual precipitation sum (blue, $21 \mathrm{yr}$ smoothing) and two indices for extreme precipitation events for the inner Alpine village Rauris. Individual years (grey bars) and $21 \mathrm{yr}$ smoothed (red). Left: sums of dayly precipitation exceeded by only $1 \%$ of all cases ( $=99 \%$ percentile) during 1961-90; right: maximum sums for five days during one year (Auer et al., 2008a, b).

1 zeigt das exemplarisch für den 90\%-Interquantilbereich und den Langfristtrend vom 19. zum 20. Jahrhundert.

Will man die Charakteristika der Trends von Klimaextremwerten zusammenfassen, muss an erster Stelle auf die noch großen Forschungsdefizite hingewiesen werden, die vor allem auf der ungenügenden Dichte von Langzeit-Messreihen beruhen. Die vorläufigen Ergebnisse aus dem Großraum Alpen zeigen einen generellen Rückgang der Temperatur-Variabilität bei gleichzeitig steigenden Temperaturen. Beim Niederschlag liegen regional und jahreszeitlich unterschiedliche Trends der Variabilität vor, es scheinen jedoch die Trends der Extremwerte analog zu denen der über längere Zeiträume akkumulierten Summen zu verlaufen. In jedem Fall sind die Langfristtrends der Veränderungen der Variabilität bzw. der Extremwerte wesentlich geringer als die Kurzfristvariabilität selbst - ein Faktum, auf das auch bei den Trends der Klimaelemente selbst bereits hingewiesen worden ist.

\section{Schlussbemerkungen}

Mit den gezeigten und diskutierten Beispielen soll einerseits das vorhandene reiche instrumentelle Datenpotential aus dem Großraum Alpen vorgestellt werden, das weltweit zu den 
Abb. 14: Zeitreiben der Variabilität des Temperaturklimas im Großraum Alpen von 1760 bis 2006: 80\% und 98\%-Quantilbereich in Zeitfenstern von 120 Monaten, die in Monatsschritten über standardisierte 30-jährig hochpassgefilterte sequentielle Monatsreiben der Temperaturen der 24 vor 1840 beginnenden instrumentellen Meßreiben der Region bewegt wurden. Dargestellt ist hier das Mittel über alle diese Langzeitreiben. Quelle: Zwischenergebnis einer laufenden Untersuchung der Arbeitsgruppe Klimawandel der ZAMG-Wien

Fig 14: Temperature variability time series in the Alpine greater area, 1760-2006. 80\% and $98 \%$ quantiles over a 120 month range, using $30 \mathrm{yr}$ high-pass filtered sepential monthly temperature records from 24 automated series starting before 1840 . The displayed data are the mean of all these long-term series. Source: Internal report, ZAMG-Vienna.

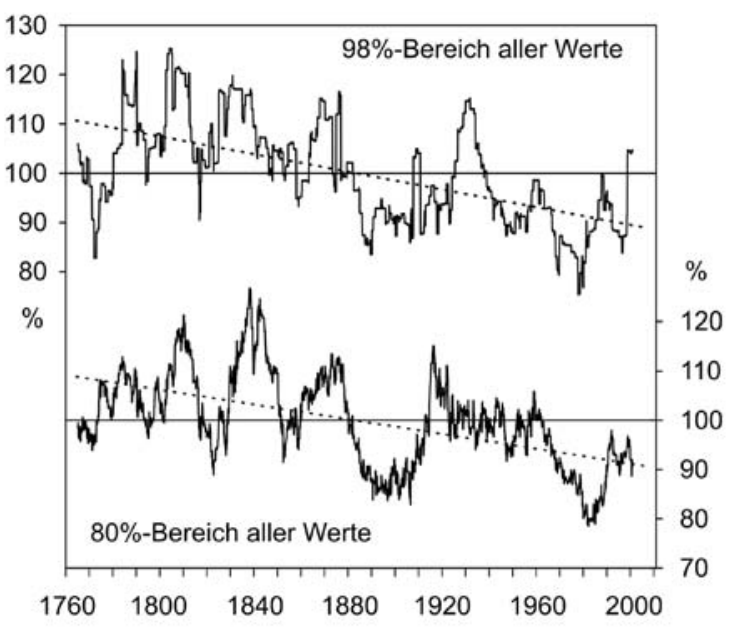

Tab.1. Prozentuelle Änderungen (Grundwert 19.Jabrbundert) der Klimavariabilität (Interquantilbereich 90\%) vom 19. zum 20. Jabrbundert auf Jahreszeiten- und Jahresbasis in drei Subregionen des Großraums Alpen fïr die Klimaelemente Temperatur und Niederschlag. Quelle: wie für Abb.14

\begin{tabular}{|c|c|c|c|c|c|c|c|c|c|c|c|c|c|c|c|c|}
\hline & $\mathrm{NE}$ & W & S & $\mathrm{NE}$ & NW & S & NE & NW & $\mathrm{S}$ & $\mathrm{NE}$ & NW & WIN & $\mathrm{NE}$ & NW & S & \\
\hline & 6.0 & -1 & -1 & -20.0 & -7. & & -3.2 & -9.7 & +0 & -7.4 & -12.0 & 2 & -28.9 & -23.8 & -11. & \\
\hline & & -3.0 & -9 & + & +8.9 & & -0.3 & +1 & -5.8 & 3.8 & +1 & 2.6 & 4.6 & +5.3 & -3 & \\
\hline
\end{tabular}

am besten aufgearbeiteten gehört und im Hinblick auf die Dichte der frühen instrumentellen Information sogar an der Spitze steht. Zusammen mit dem gerade in dieser Region hohen Potential an Proxy-Klimainformationen, ergibt das sehr gute Möglichkeiten zur indirekten Klimarekonstruktion. Diese gilt es, durch interdisziplinäre Zusammenarbeit in Hinkunft noch besser zu nutzen.

Andererseits wurde aufgezeigt, dass auch in der instrumentellen Domäne von „Rekonstruktion“ gesprochen werden muss, da die Originaldaten per se nicht der tatsächlichen Klimaentwicklung über zweieinhalb Jahrhunderte entsprechen. Zahlreiche Gründe wurden angeführt, die in jeder gemessenen Zeitreihe zu Inhomogenitäten führen können, die die wahre Klimainformation verdecken. Die beiden Beispiele zeigten eine bereits erfolgreich abgeschlossene Teilarbeit an der Dauerbaustelle der HISTALP Klimadatenbasis der ZAMG (die Korrektur des EIP-Bias) und eine, die erst teilweise bewältigt ist (die Homogenisierung von Tagesdaten), der aber im Hinblick auf die steigenden Ansprüche der Öffentlichkeit (Extremwertdebatte) und der Wissenschaft selbst (Proxy- und Impactmodellierung mit Tageswerten) zunehmend größere Bedeutung zukommt. 


\section{Danksagung}

Es konnte auf die Grundlagenarbeiten einer Reihe abgeschlossener und noch laufender Forschungsprojekte der Klimaabteilung der Zentralanstalt für Meteorologie und Geodynamik (ZAMG) Wien zurückgegriffen werden: CLIVALP (FWF, P15076-N06), ALPIMP (EU-FP5, EVK-CT-2002-00148), FORALPS (Interreg-Alpine-IIIb Alpine Space, I/ III/3.1/21), A Tale of two Valleys (BMWF-Programm proVision, 133-111), HISTALP2007 (ZAMG-BMWf 4426/07-9), COST ES Projekt 0601 HOME, HOMDAY (ZAMGBMWF 4426/07-23).

Für die Förderung sei an dieser Stelle nochmals gedankt. Ganz besonders gilt aber der Dank den vielen bekannten und unbekannten Beobachtern, die in langjähriger, oft schlecht entlohnter Teilarbeit den HISTALP-Datensatz geschaffen haben.

\section{Literatur}

Aguilar E, Auer I, Brunet M, Peterson TC, Wieringa J (2003) Guidelines on Climate Metadata and Homogenization. World Climate Programme Data and Monitoring WCDMP 53, WMO-TD 1186, WMO. Geneva

Auer I, Jurkovic A, Orlik A, Böhm R, Korus E, Sulis A, Marchetti A, Dolinar M, Nadbath M, Vertacnik G, Vicar Z, Pavcic B, Geier G, Rossi G, Leichtfried A, Schellander H, Gabl K, Zardi D, 2008a. High quality climate data for the assessment of Alpine climate, its variability and change on regional scale - Collection and analysis of historical climatological data and metadata. Technical Report of WP5, Interreg-IIIb- Alpine Space Project Foralps, I/III/3.1/21, 67 pages plus 7 annexes

Auer I et al., 2008b. Endbericht des BMWF- proVision Projekts "A Tale of two Valleys. In Arbeit

Auer I, Böhm R, Jurkovic A, Lipa W, Orlik A, Potzmann R, Schöner W, Ungersböck M, Matulla C, Briffa K, Jones PD, Efthymiadis D, Brunetti M, Nanni T, Maugeri M, Mercalli L, Mestre O, Moisselin J-M, Begert M, Müller-Westermeier G, Kveton V, Bochnicek O, Stastny P, Lapin M, Szalai S, Szentimrey T, Cegnar T, Dolinar M, Gajic-Capka M, Zaninovic K, Majstorovic Z, Nieplova E (2007) HISTALP - Historical instrumental climatological surface time series of the greater Alpine region 1760-2003. Int J Climatol 27: 17-46

Auer I, Böhm R, Jurkovic A, Orlik A, Potzmann R, Schöner W, Ungersböck M, Brunetti M, Nanni T, Maugeri M, Briffa K, Jones P, Efthymiadis D, Mestre O, Moisselin JM, Begert M, Brazdil R, Bochnicek O, Cegnar T, Gajic-Capka M, Zaninovic K, Majstorovic Z, Szalai S, Szentimrey T (2005) A new instrumental precipitation dataset in the greater alpine region for the period 18002002. Int J Climatol 25: 139-166

Bard E, Raisbeck G, Yiou F, Jouzel J, 2000. Solar irradiance during the last 1200 years based on cosmogenic nuclides. TELLUS B 52: 985-992

Böhm R, Jones PD, Hiebl J, Frank D, Brunetti M and Maugeri M (2008) The early instrumental warm-bias: a solution for long central European temperature series 1760-2007. Climatic Change, in review 


\section{Klimawandel in Österreich}

Böhm R, Auer I, Brunetti M, Maugeri M, Nanni T and Schöner W (2001) Regional temperature variability in the European Alps 1760-1998 from homogenized instrumental time series. Int J Climatol 21: 1779-1801

Brohan P, Jennedy J, Harris I, Tett SFB and Jones PD (2006) Uncertainty estimates in regional and global observed temperature changes: a new dataset from 1850. Journal of Geophysical Research 111 D12106, doi: 10.1029/2005JD006548

Büntgen U, Frank DC, Nievergelt D, Esper J (2006) Summer temperature variations in the European Alps: AD 755-2004. Journal of Climate 19: 5606-5623

Crowley TJ, 2000. Causes of Climate Change Over the Past 1000 Years. Science 289: 270-277

Frank D, Büntgen U, Böhm R, Maugeri M, Esper J (2007) Warmer early instrumental measurements versus colder reconstructed temperatures: shooting at a moving target. Quaternary Science Reviews 26: 3298-3310

Geiger, R, Aron RH and Todhunter P (2005) The Climate near the Ground. Vieweg, Braunschweig, 528 pages

Hammerl C, Lenhart W, Steinacker R und Steinhauser P (Hg.) (2001) Die Zentralanstalt für Meteorologie und Geodynamik 1851-2001 - 150 Jahre Meteorologie und Geophysik in Österreich. Leykam, Graz, 818 Seiten plus eine Klima- und eine Geophysik-CD.

Hiebl J (2006) The early instrumental climate period (1760-1860) in Europe. Evidence from the Alpine region and Southern Scandinavia Diploma thesis, Geogr Inst, University of Vienna, 103 pages

Jones PD and Moberg A (2003) Hemispheric and large-scale surface air temperature variations: An extensive revision and an update to 2001. J Climate 16, 206-223

Lean J, 2000. Evolution of the Sun's Spectral Irradiance since the Maunder Minimum. Geophysical Research Letters 27: 2425-2428

Della-Marta P and Wanner H (2006) A method of homogenizing the extremes and mean of daily temperature measurements. Journal of Climate 19: 4179-4197

Moberg A, Bergström H (1997) Homogenization of Swedish temperature data. Part III: the long temperature records from Stockholm and Uppsala. Int J Climatol 17: 667-699

Moberg A, Alexandersson H, Bergström H and Jones PD (2003) Were southern Swedish temperatures before 1860 as warm as measured? Int J Climatol 23: 1495-1521

Peterson TC, Easterling DR, Karl TR, Groisman P, Auer I, Böhm R, Plummer N, Nicholis N, Torok S, Vincent L, Tuomenvirta H, Salinger J, Förland EJ, Hanssen-Bauer I, Alexandersson H, Jones PD, Parker D (1998) Homogeneity Adjustments of In Situ Climate Data: A Review. Int J Climatol 18: $1493-1517$

Robertson A, Overpeck J, Rind D, Mosley-Thompson, Zielinski G, Lean J, Koh D, Penner J, Tegen I, Healy R, 2001. Hypothesized climate forcing time series for the last 500 years. Journal of Geophysical Research - Atmosphere 106: D14 p14783 (2000JD900469)

v. Rudloff H (1967) Die Schwankungen und Pendelungen des Klimas in Europa seit dem Beginn der regelmäßigen Instrumenten-Beobachtungen (1670). Vieweg, Braunschweig, 370 pages

Zorita E, von Storch H, Gonzalez-Rouco FJ, Cubasch U, Luterbacher J, Legutke S, Fischer-Bruns I, Schlese U, 2004. Climate evolution in the last five centuries simulated by an atmosphere-ocean model: global temperatures, the North Atlantic Oscillation and the Late Maunder Minimum. Meteorologische Zeitschrift 13: 271 - 289 


\title{
Das Klima der nächsten 100 Jahre
}

\author{
Christoph Matulla \\ Zentralanstalt für Meteorologie und Geodynamik, Wien
}

Abstract

The climate of the century ahead

'Scientists often fail to predict the weather just a few days ahead, so they're certainly not capable to tell what's going to happen within the next century!'

Have you already heard such arguments? At first it is important to separate weather from climate. However, to answer to the above argument at once - Yes, it is possible to tell the probable climate of the decades to come. Such statements rely on requirements how mankind may progress in the future. There are uncertainties involved in the generation of future climate projections one has to be aware of and which have to be discussed. This paper demonstrates a chain of the very basics necessary to derive local scale climate scenarios and presents some findings related to Austria.

,Die Forscher können oft nicht mal das Wetter der nächsten 10 Tage zutreffend vorhersagen, also geht das erst recht nicht für die nächsten 100 Jahre!

Haben Sie derartige Argumente schon gehört? Zuerst ist es wichtig die Begriffe Wetter und Klima auseinanderzuhalten. Um aber gleich zu dem oben genannten Argument Stellung zu beziehen - Doch, man kann das Klima kommender Dekaden, basierend auf Annahmen, wie sich die Menschheit entwickelt, simulieren. Selbstverständlich sind derartige Abschätzungen, die auch Projektionen genannt werden, mit Unsicherheiten auf verschiedenen Ebenen verbunden, die zu berücksichtigen und kommunizieren sind. Dieser Artikel listet eine Kette an Basisbegriffen der Klimamodellierung vor, die die Erstellung von lokalskaligen Klimaszenarien ermöglicht und stellt einige Ergebnisse für Österreich vor.

\section{Wetter und Klima - bier soll „König Fußball“ bei der Motivation helfen:}

Grundlegend ist die Verschiedenheit von Wetter und Klima. Vielleicht lässt sich diese anhand einer Fußballmannschaft (nennen wir die Gruppe ,Jubel') illustrieren. Sagen wir, Jubel verliert ein Match gegen eine andere Mannschaft. Am Ende der Fußballsaison aber gewinnt Jubel die Meisterschaft. Beides ist möglich: Jubel kann ab und zu ein Spiel verlieren und dennoch Meister werden. Wenn wir das nun auf 
Wetter und Klima umlegen, dann entsprächen die einzelnen Matches dem Wetter und der Meistertitel dem Klima. Das Wetter in einem für viele Sonnenstunden berühmten Badeort kann mal schlecht sein, dennoch kann man generell dort fabelhaft urlauben. Anderenfalls wäre der Badeort nicht berühmt, jedenfalls nicht für sein gutes Badewetter. Natürlich hinken die Vergleiche.

Statistisch gesehen ist das Wetter eine Realisation des Zustandes des Klimasystems über einige Tage. Klima hingegen ist die Statistik des Wetters über typischerweise einige Jahrzehnte. Die statistische Beschreibung von Größen wie Temperatur, Niederschlag oder Luftdruck erfolgt über deren Verteilungen, also Mittelwerte, Standard-Abweichungen, Perzentile etc. Wenn sich diese Lagemaße im Laufe der Zeit ändern, spricht man von Klimaänderung.

Genauso kann es Jubel gehen. Es könnte sein, dass das Team von den 1930ern bis in die 1960er eine erfolgreiche Mannschaft war, während es von den 1970ern bis in die 2000er keine überragenden Leistungen erbracht hat. Man könnte salopp sagen, das Klima in der ersten Periode war besser als das in der zweiten. Die gewonnenen Spiele waren in der ersten Periode zahlreicher, das Torverhältnis günstiger, kurz - die Statistiken der beiden Perioden unterscheiden sich.

\section{Das Klimasystem und seine Antriebe}

Das Klimasystem besteht aus verschiedenen Komponenten: der Atmosphäre, der Hydrosphäre (flüssiges Wasser), der Kryosphäre (Schnee und Eis), der Biosphäre und der Lithosphäre.

Die Sonne beliefert das Klimasystem mit Energie und löst innerhalb der Komponenten physikalische und chemische Prozesse aus. Es kann sich z.B. eine Zyklonenfamilie in der Atmosphäre über dem Nordatlantik ausbilden. Auch zwischen den Komponenten gibt es Wechselwirkungen - Wind streicht über den Ozean und erzeugt Wellen. Der Energieeintrag der Sonne variierte in den letzten 1000 Jahren um 2 bis $3 \mathrm{~W} / \mathrm{m}^{2}$ um den Wert $1365 \mathrm{~W} / \mathrm{m}^{2}$.

Vulkanausbrüche schleudern bei heftigen Eruptionen Aerosole in hohe Schichten der Atmosphäre. Die Aerosole reflektieren die Solarstrahlung ins All zurück und können sich, speziell wenn sie die Stratosphäre erreichen, dort einige Jahre halten. Vulkanausbrüche haben eine abkühlende Wirkung, die in der Größenordnung von einigen $\mathrm{W} / \mathrm{m}^{2}$ liegt.

Die chemische Zusammensetzung der Atmosphäre beeinflusst, wie viel der eingestrahlten solaren Energie im Klimasystem verbleibt. Eine zunehmende Dichte von Treibhaus- 
gasen in der Atmosphäre hindert die von der Erde ausgehende langwellige Strahlung daran ins All zu gelangen und hat eine Erwärmung des Klimasystems zur Folge.

Eine vollständigere, sich an den interessierten Laien wendende Beschreibung der Klimaantriebe findet sich etwa in Böhm (2008). Die Lektüre dieses Werkes ist jedenfalls ein vergnügliches und lohnendes Unterfangen.

\section{Die Modellierung des Klimas}

Die Prozesse, die innerhalb oder zwischen den Komponenten des Klimasystems stattfinden, gehorchen physikalischen und chemischen Gesetzen. Diese können vereinfacht und in Computercode übersetzt werden. Ein Programm, das die Prozesse in Abhängigkeit von den Antrieben näherungsweise simuliert, wollen wir in der Folge Klimamodell nennen. Der Betrieb eines Klimamodells ist rechentechnisch und wissenschaftlich eine große Herausforderung und erfolgt an Höchstleistungsrechenzentren. Es gilt, in Abhängigkeit von der Fragestellung, die Balance zu finden zwischen dem nötigen Detailgrad der physikalisch-mathematischen Beschreibung der Prozesse im Klimamodell und dem zu bewältigenden Rechenaufwand. Über den Globus verteilt gibt es einige Rechenzentren (z.B. das Deutsche Klimarechenzentrum ,DKRZ ' in Hamburg, das Canadian Centre for Climate Modelling and Analysis ,CCCma' in Victoria oder das englische Hadley Center in Exeter), die verschiedene Klimamodelle betreiben (z.B. das deutsche globale Klimamodell ,ECHAM' in Hamburg, das kanadische Modell ,CGCM“' in Victoria oder das ,HadCM' in Exeter). Wenn im Folgenden die Rede vom deutschen oder englischen Klimamodell die Rede sein wird, ist ECHAM oder HadCM gemeint.

Klimamodelle geben in Abhängigkeit von den Antrieben angenähert die Prozesse wieder, die in und zwischen den Komponenten ablaufen. So etwa, welche Energiemengen über die Ozeane oder die Atmosphäre von den niederen Breiten in Richtung der Pole transportiert werden, oder wie groß der Anteil der Sonnenstrahlung ist, der direkt in den Weltraum reflektiert wird, bzw. wie groß der Anteil der langwelligen Strahlung ist, der durch den anthropogenen Treibhausgaseffekt im Klimasystem zurückgehalten wird. Für die Vergangenheit kann man den zeitlichen Verlauf der Antriebe entweder aus direkten Messungen oder aus Rekonstruktionen (durch Auswertungen ,indirekter Klimazeugen', worüber wir in den anderen Beiträgen dieses Buches viel lernen können) erhalten. Ein Klimamodell, das aus den rekonstruierten Antrieben bestimmte Elemente des vergangenen Klimas reproduzieren kann, kann hinsichtlich dieser Aspekte als nützlich betrachtet werden. Ein Beispiel 

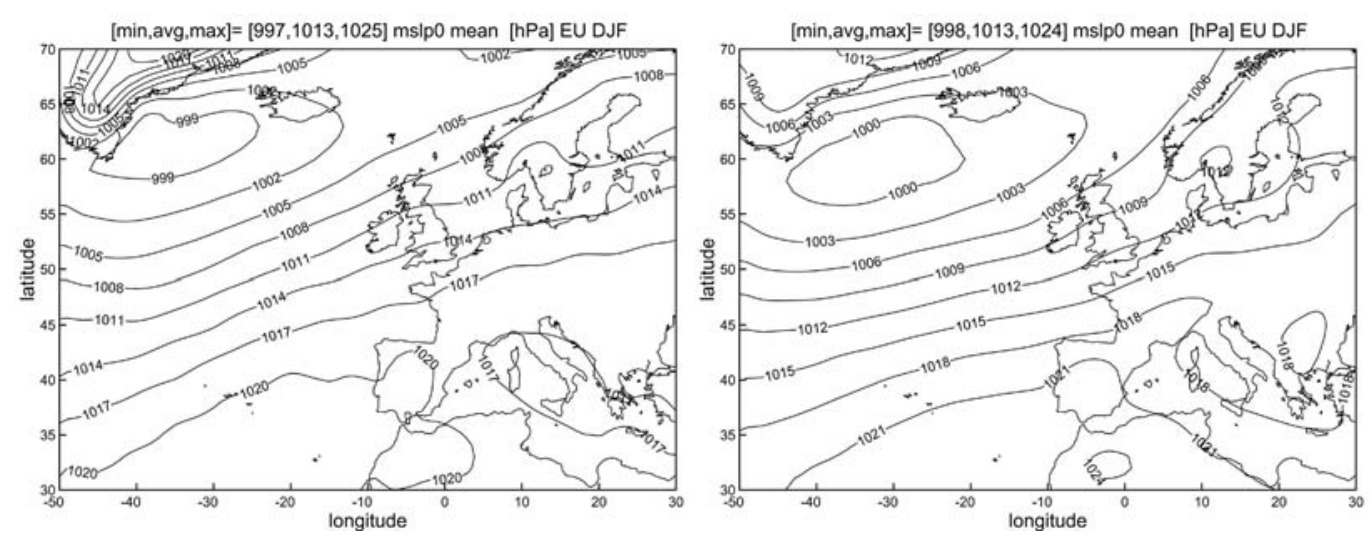

Abb. 1: Mittlerer Luftdruck auf Meeresniveau während der Wintersaison. Links: aus Beobachtungsdaten (19482005); rechts: aus einem Kontrolllauf des deutschen Klimamodells ECHAM.

Fig. 1. Mean air pressure at sea altitude during winter season. Left: from observations (1948-2005); rigth: from a control run of the German climate model ECHAM.

dafür ist die Verteilung des Drucks auf Meeresniveau (siehe Abbildung 1). Das linke Bild in Abbildung 1 zeigt die Verteilung des mittleren Luftdrucks auf Meeresniveau, berechnet aus Beobachtungsdaten der Wintermonate der Periode von 1948 bis 2005. Das rechte Bild zeigt dasselbe Feld, allerdings nicht basierend auf Beobachtungsdaten, sondern auf einem so genannten Kontrolllauf des deutschen Klimamodells ECHAM. Bei einem Kontrolllauf werden dem Klimamodell historische Antriebe vorgegeben. Treibhausgaskonzentrationen werden auf einem bestimmten, für die betrachtete Periode repräsentativen Niveau konstant gehalten. Soll das Klima vor Beginn der Industrialisierung simuliert werden, hält man die Kohlendioxidkonzentration auf etwa $280 \mathrm{ppm}$.

Der Luftdruck ist eine weitere für das Klima entscheidende Größe. Seine Verteilung bestimmt die Zugbahnen von Luftmassen und legt damit regionale klimatische Verhältnisse fest. Für weite Teile Europas ist die Druckverteilung im Atlantik (siehe Abbildung 1) von signifikanter Bedeutung. Hoher Luftdruck über den Azoren und tiefer Luftdruck über Island bestimmen, wie Tiefdruckgebiete nach Europa geführt werden. Ist der Luftdruck über Island tiefer und über den Azoren höher als im Durchschnitt, dann ist eine Zunahme der Sturmtätigkeit in Nordeuropa wahrscheinlich. Im umgekehrten Fall gilt das für südlichere Teile Europas. Wegen seiner Bedeutung für das Klima sollten Klimamodelle, die zur Generierung von Klimaprojektionen (Szenarien für die Zukunft) Verwendung finden, die Luftdruckverhältnisse gut simulieren können. In Abbildung 1 ist das der Fall. In den Beobachtungen 
wie in der Simulation findet sich der für den Winter charakteristische zonal ausgeprägte Zirkulationszustand. Ein Hochdruckgebiet mit Kerndruck über $1020 \mathrm{hPa}$ im Großraum um die Azoren und ein Tiefdruckgebiet mit einem Kerndruck unter 1000 $\mathrm{hPa}$ süd-östlich von Island. Im zentralen Mittelmeerraum ist ein relativer Tiefdruck von ca. $1017 \mathrm{hPa}$ angedeutet. Großräumig verlaufen die Isobaren in beiden Bildern von Süd-West nach Nord-Ost. Die Luftdruckunterschiede liegen in beiden Fällen bei über $20 \mathrm{hPa}$. Insgesamt resultiert eine Westströmung mit südlicher Komponente. Für die Zirkulation wesentliche Aspekte werden von ECHAM in Stärke wie Lage der Aktionszentren realitätsnahe wiedergegeben.

\section{Szenarien für die Entwicklung der Menschbeit}

Was hat nun die Fußballmannschaft Jubel mit Klimaprojektionen für die Zukunft zu tun? Nehmen wir an, wir sind Mitglieder des Vereinspräsidiums und stehen in Verhandlungen um lukrative Sponsorenverträge. Es sieht so aus, als könnte langfristig viel Geld verfügbar gemacht werden. Sagen wir, für die nächsten 3 Dekaden ist Jubel monetär die am besten ausgestattete Mannschaft der Liga. Außerdem wurde in den vergangenen Jahren richtig gute Nachwuchsarbeit geleistet. Das bedeutet, hochbegabte Spieler aus der eigenen Jugendmannschaft, die nicht teuer ,eingekauft ${ }^{6}$ werden müssen. Das Präsidium hat also gute Gründe anzunehmen, dass die Statistik der nächsten 30 Jahre eher an die erfolgreiche erste Phase im 20. Jahrhundert erinnern wird (1930er-1960er, siehe Seite 1) als an die weniger erfreuliche zweite Periode. Es ist natürlich nicht möglich, jedes einzelne Spiel in der Zukunft vorherzusagen, dennoch sind die Rahmenbedingungen so, dass Jubel wahrscheinlich gute Platzierungen in den künftigen Meisterschaften erreichen wird.

Die eingangs erwähnten Klimaantriebe sind Rahmenbedingungen für das Klima. Diese verändern sich mit der Zunahme der Weltbevölkerung und deren Energieverbrauch sowie durch die technische und soziale Entwicklung. Dergleichen abzuschätzen ist ein komplexes Unterfangen, denn es gibt - mit Ausnahme der Bevölkerungszahlen - kaum Vorstellungen, wie sich die Gesellschaften der Erde in den nächsten Jahrzehnten entwickeln werden. Eine Strategie, dennoch zu Aussagen zu gelangen, besteht darin, mehrere möglichst verschiedene wirtschaftliche, gesellschaftspolitische und andere Entwicklungen anzunehmen und darauf basierend Zukunftsszenarien abzuschätzen. Diesen Zugang hat das Intergovernmental Panel on Climatic Change (IPCC) gewählt. Dabei werden von Fachleuten Szenarien, wie sich die Weltbevölkerung, deren Energiebedarf etc. entwickeln könnte, entworfen. 

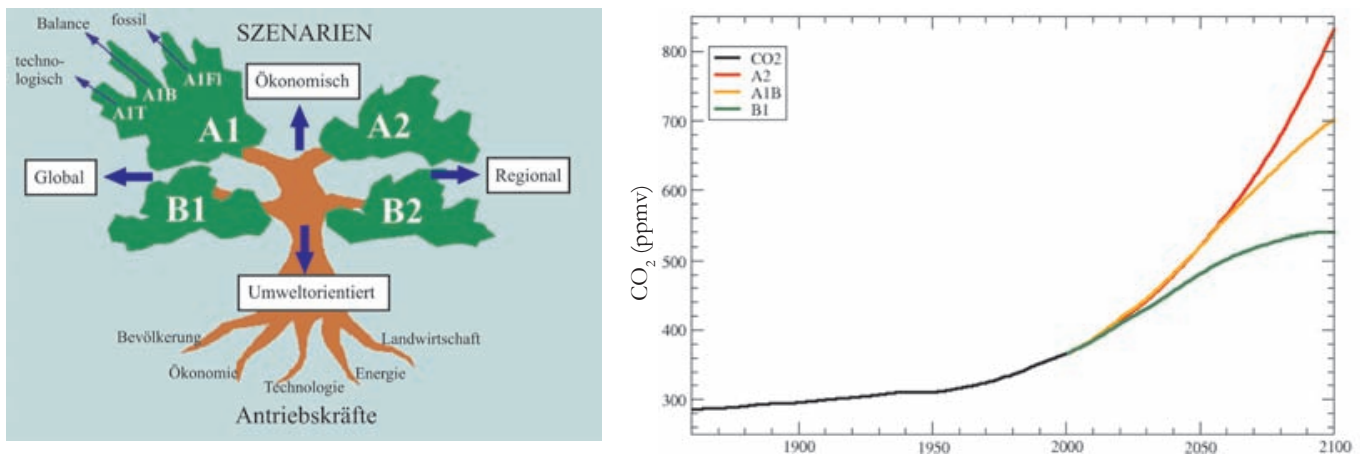

Abb.2: Der IPCC Szenarien-Baum (links) stellt die die Rabmenbedingungen dar unter welchen die Entwicklung der Menschbeit beschrieben wird. Rechts sind die mit solchen Szenarien verbundenen Projektionen der $\mathrm{CO}_{2}$ Konzentrationen dargestellt (Quelle: IPCC).

Fig. 2. The IPCC scenario tree (left) depicts the conditions used to describe the future of mankind. Right: projections of $\mathrm{CO}_{2}$ concentrations depending on different scenarios (source: IPCC).

Abbildung 2 stellt den so genannten IPCC Szenarienbaum dar. Die Pole, in deren Spannungsfeld die Szenarien liegen, sind einerseits die wirtschaftliche Orientierung (ökonomisch versus ökologisch) und andererseits die politische Ausrichtung (internationale Vernetzung d.h. gute Kommunikation und schneller Technologietransfer versus Abschottung und Blockbildung).

Die Szenarien-Familien und einzelne Szenarien sind detailliert im Second Report on Emission Szenarios (SRES, IPCC 2001) zusammengefasst. Hier folgen nur einige Bemerkungen zu Szenarien, auf die sich der Text noch beziehen wird.

Der A1 Zweig beschreibt eine Zukunft mit sehr ausgeprägtem Wirtschaftswachstum, einer Bevölkerungszahl, die in der Mitte des 21. Jahrhunderts ihren Höchststand (8.7 Mrd.) erreicht und dann sinkt, und einer schnellen Verbreitung effizienter Technologien. Die Menschheit wächst zusammen, d.h. regionale Unterschiede bei den Einkommen, in kultureller und sozialer Hinsicht und in der technologischen Entwicklung gleichen sich weitgehend aus. Es werden drei Untergruppen unterschieden: die A1Fl Gruppe mit einer intensiven Nutzung fossiler Brennstoffe; die A1T Gruppe mit starker Nutzung nicht-fossiler Energieträger und die A1B Gruppe, welche für das Bedienen des Energiebedarfes aus einem ausbalancierten Mix verschiedener Energiequellen steht.

Der B1 Zweig umfasst Szenarien, die global orientiert sind, wie die des A1 Zweiges, jedoch mit einer Lenkung der Wirtschaftsentwicklung in den Dienstleistungs- und Informationssektor, mit einem geringeren Materialverbrauch und der Einführung sauberer und Ressourcen schonender Technologien. Das Ziel ist Nachhaltigkeit 
im wirtschaftlichen, sozialen und ökologischen Bereich, sowie eine balancierte Wohlstandsverteilung. Das heißt hohes Umwelt- und Sozialbewusstsein. Gemeinschaftswerte setzen sich gegen Individualismus durch, was durch starke politische Steuermechanismen erwirkt wird. Der Energiebedarf wird mit treibhausgasarmer Technologie gedeckt. Die Bevölkerungszahl entwickelt sich nahezu ident zu der in A1 beschriebenen.

A2 Szenarien gehen von einer heterogenen Welt aus. Lokale Besonderheiten bleiben bestehen und die Bevölkerung nimmt insgesamt zu (über 15 Mrd. bis 2100). Ökonomische und technologische Entwicklung, das Wachstum des Bruttosozialprodukts sind regional ungleich und im Vergleich mit den anderen Szenariozweigen verzögert.

Diese Entwicklungsszenarien der Menschheit werden dann in Emissionsszenarien umgelegt, die als Funktion der Zeit beschreiben, wie sich die Treibhausgaskonzentrationen in der Atmosphäre entwickeln könnten (Abbildung 2, rechtes Bild).

\section{Globale Klimaänderungsszenarien}

Oben beschriebene Szenarien über den künftigen Verlauf der Klimaantriebe werden verwendet, um mit Hilfe von Klimamodellen global die Reaktion des Klimasystems auf diese Szenarien abzuschätzen. Damit werden, wenn - dann' Antworten gegeben (,wenn Emissionsszenario X - dann Klimareaktion $Y^{\urcorner}$). Mit anderen Worten, es geht darum, die Konsequenzen möglicher gesellschaftlicher Entwicklungen abzuschätzen und damit eine Basis für politische Entscheidungen zu schaffen. Es kann also beurteilt werden, ob die Entwicklung, die im B1 Szenario beschrieben ist, in der Zukunft auf eine andere großräumige Temperaturverteilung hinführt als die für A1 angenommene Entwicklung. Abbildung 3 zeigt die Veränderungen der Jahresmitteltemperaturen aufgrund der oben beschriebenen Szenarien für drei Perioden im 21. Jh. verglichen mit der Periode 1980-1999. Die Abbildungen sind Mittelwerte über viele Simulationen, die mit verschiedenen globalen Klimamodellen weltweit generiert worden sind.

Daraus ist eine generelle Temperaturzunahme zu erkennen, die über den Kontinenten in den hohen Breiten stärker ausgeprägt ist als über den Ozeanen. Die Periode 2011-2030 unterscheidet sich nicht wesentlich hinsichtlich der Szenarien, in der dritten Periode (2080-2099) sind die Unterscheide aber signifikant. Das A2 Szenario zeigt in hohen Breiten Temperaturzunahmen von über $7 \mathrm{Grad}$ während der Temperaturzuwachs dem B1 Szenario folgend in dieser Region etwa 4 Grad 


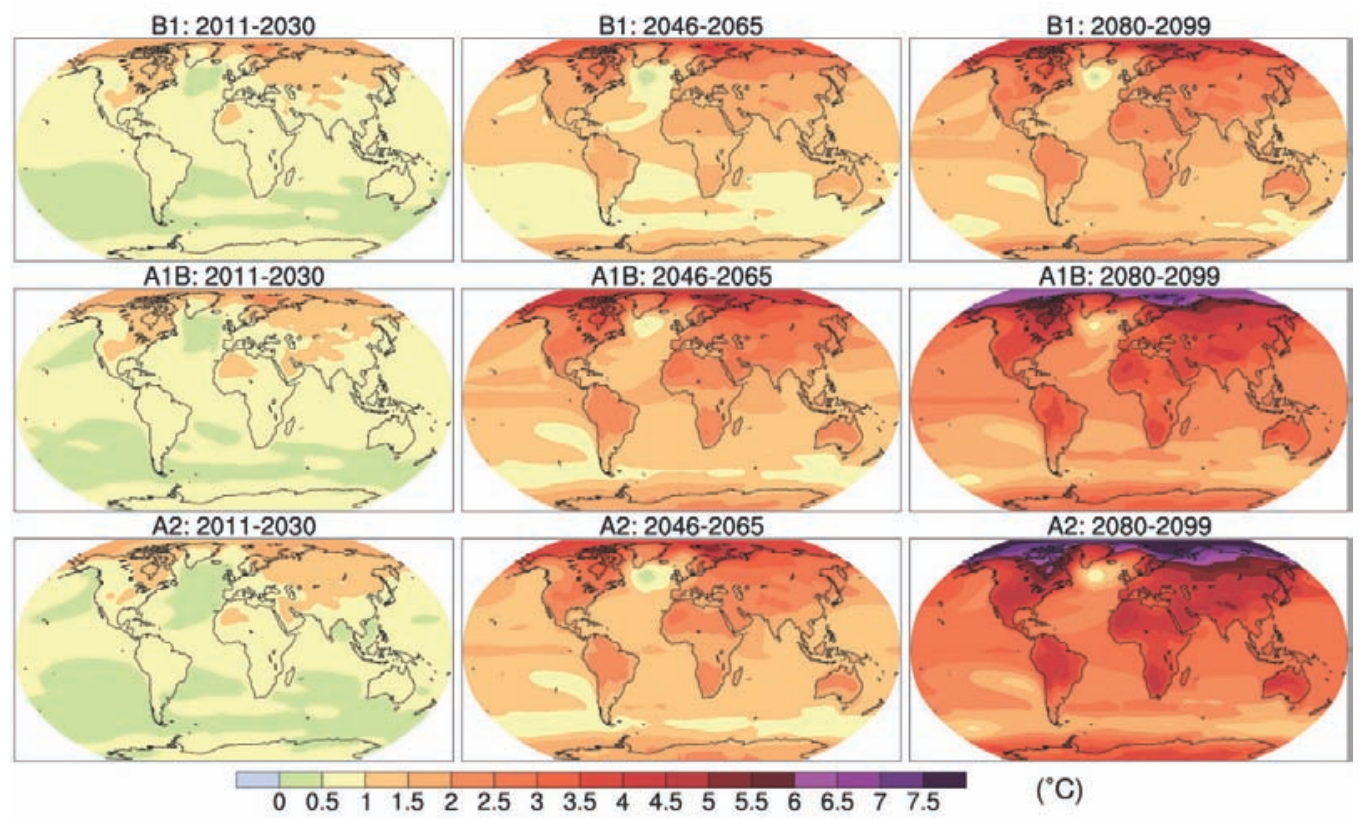

Abb. 3: Mittelwerte der jährlichen Temperaturzunabme anhand von Klimamodell-Simulationen für die Szenarien B1 (oben), A1B (Mitte) und A2 (unten) und für drei Perioden im 21 Jh. (links: 2011-2030, Mitte: 2046-2065, rechts: 2080-2099). Die Zunabmen sind relativ zu der Periode 1980-1999 (aus: IPCC 2007).

Fig. 3: Mean values of annual temperature increase bbased on in climate model simulations for scenarios B1 (top), A1B (middle) and A2 (below) and for 3 periods in the 21st century (left: 20012030; middle: 2046-2065; right: 2080-2099). All increases in comparison to the period 1980-1999 (from IPCC 2007).

ausmacht. Grundsätzlich unterscheiden sich die Projektionen mehr im Ausmaß der Temperaturzunahme als in deren räumlichem Muster.

\section{Regionale Klimaänderungsszenarien}

Ergebnisse globaler Klimamodelle wie in Abbildung 3 sind auf einer Zeitskala von einigen Dekaden (einer Klimaperiode) und räumlich für Kontinente zu interpretieren. Regionale Auswirkungen des möglichen Klimawandels können mit globalen Klimamodellen etwa auf Grund ihrer groben Auflösung nicht modelliert werden. Um die Reaktion von Ökosystemen auf veränderte Klimabedingungen bewerten zu können, wird aber in der Regel räumlich hochaufgelöstes Datenmaterial benötigt. Methoden, die Ergebnisse von globalen Klimamodellläufen (horizontale Auflösung von rund $100 \mathrm{~km}$ ) mit regionaler Information konsistent verbinden, nennt man ,Downscalingverfahren' (von Storch et al. 1993, von Storch 1995, Zorita und von Storch 1999). Dabei wird vorausgesetzt, dass die regionalen Klimabedingungen von 
den großskaligen Randbedingungen abhängig sind. Aus ,downgescalten' (herunterskalierten) Szenarien können dann regionale Auswirkungen beurteilt werden. Grob werden beim Downscaling zwei Zugänge unterschieden:

Dynamisches Downscaling orientiert sich an den bekannten physikalischen Gesetzen, die Vorgänge auf lokaler Skala im Klimasystem beschreiben. Diese werden mit einem so genannten regionalen Klimamodell (mit hoher Auflösung von einigen Kilometern) berechnet. Ein regionales Klimamodell beschreibt für eine begrenzte Region (z.B. Mitteleuropa) physikalische Prozesse im Klimasystem, die von einem globalen Klimamodell nicht simuliert werden können. Dafür benutzt es die Randbedingungen, die vom globalen Klimamodell vorgegeben werden. Regionale Modelle werden gewissermaßen in das Gitter des globalen Klimamodells ,hineingehängt‘.

Empirisches Downscaling bedient sich Messreihen auf der Skala der globalen Modelle und der Skala, die man aufzulösen wünscht (z.B. Stationsdaten). Die Reihen werden zueinander in Beziehung gesetzt, und mit statistischen Methoden wird ein Zusammenhang zwischen ihnen abgeleitet. Dieser ermöglicht es, in Abhängigkeit von großskaligen Klimaänderungs-Simulation auf den künftigen Zustand der kleinskaligen Variable, d.h. auf lokaler Ebene, zu schließen.

Abbildung 4 zeigt das Schema des dynamischen Downscalings. Das linke Bild steht stellvertretend für ein Klimaszenario, gerechnet mit einem globalen Klimamodell, basierend auf einem der oben beschriebenen Emissionsszenarien. Das regionalskalige Szenario (rechtes Bild) wurde am DKRZ mit dem regionalen Klimamodell CLM (= das Community-Model der deutschen Klimaforschung) aus dem globalen Szenario herunterskaliert. Im unteren Bild sind die auf zwei ,downgescalten' Szenarien beruhenden Temperaturveränderungen für die Region Tirol gezeigt.

Beide Formen des Downscalings hängen kritisch von der Fähigkeit des globalen Klimamodells ab, das großskalige Klimageschehen realistisch wiederzugeben. Statistische Verfahren benötigen (relativ) wenig Computerressourcen, kein detailliertes Wissen über die Beschaffenheit des Untergrunds und der lokalskaligen physikalischen Prozesse. Daher stehen diese Verfahren einem weiten Kreis von Anwendern offen. Ein guter Überblick über Downscaling findet sich in IPCC (2001).

Abbildung 5 zeigt im unteren Bild die Temperaturverteilung, die aus einem globalen Klimalauf mit empirischen Downscaling abgeleitet worden ist (Matulla et al. 2004). Der globale Lauf wurde mit ECHAM unter dem so genannten ,business as usual' Szenario (,weiter wie bisher') als Antrieb erzeugt. Das ,business as usual' Szenario ist, den Verlauf der künftigen $\mathrm{CO}_{2}$ Konzentration betreffend, nahe am A1B Szenario. 


\section{Regionalskaliges Szenario \\ DOWNSCALING (z.B. mit regionalem Klimamodell)}
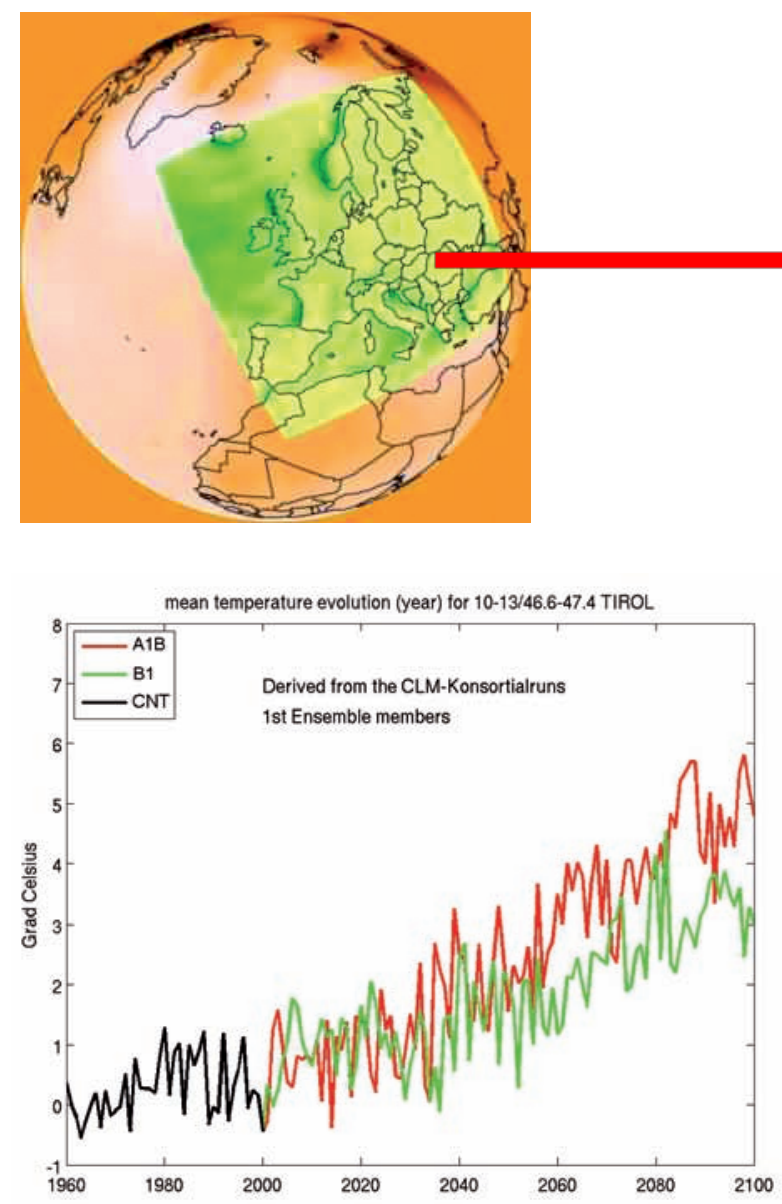

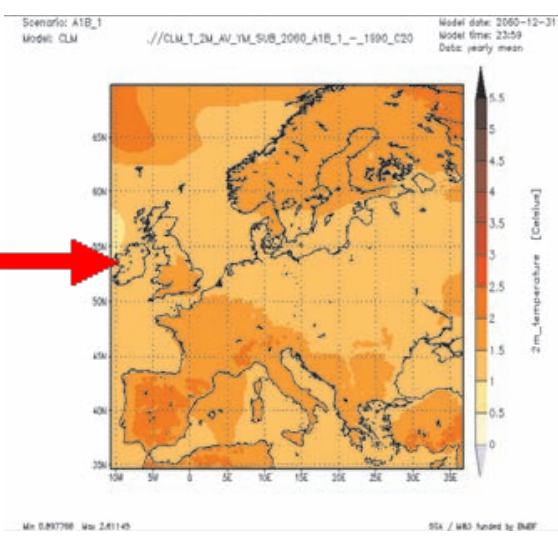

Abb. 4: Prinzip des Downscalings. Linkes Bild (Quelle: Vortrag von H. von Storch): ein globales Szenario, rechtes Bild (Quelle: $S G A / M P I):$ ein downgescaltes Szenario, æ.B. mit dem regionalen Modell CLM oder empirisch; unten: Temperaturzunabmen für Tirol, abgeleitet aus zwei mit CLM downgescalten Szenarien (A1B und B1). CNT steht fuer Kontrolllauf.

Fig. 4. Priciple of downscaling. Left (source: presentation of H. von Storch): a global scenario; right (source: SGA/ MPI): a downscaled scenario, for instance with the regional CLM model or empirically; below: temperature increase for Tyrol, derived from two downscaled scenarios (A1B and B1) by use of CLM. CNT stands for control run.

Die in Abbildung 5 dargestellte Veränderung der Temperatur in Österreich würde sich deutlich auf den hydrologischen Zyklus und das Abflussverhalten inneralpiner Flüsse auswirken. Das Temperaturniveau steuert, wie viel an Niederschlag in welcher Region als Regen oder Schnee fällt. Das wirkt sich auf die Akkumulation von Schnee und das Abflussverhalten aus. Höhere Wintertemperaturen verringern die Schneedecke, die im Winter aufgebaut wird, und damit die Menge an zu Verfügung stehendem Schmelzwasser, steigern die Evaporation und reduzieren so die Abflussmenge im Frühjahr. 
Abb. 5: Beispiel für ein regionalisiertes Temperaturszenario für die Wintersaison Matulla et al. 2004). Die obere Abb. (a) zeigt die Temperatur abgeleitet aus Beobachtungen (1961-1990), die untere Abb. für 2041-2050 (b) die Temperaturen aus dem, business as usual' Szenario abgeleitet aus dem mit dem Klimamodell ECHAM realisierten globalen Szenario. In ganz Österreich werden Temperaturzunabmen modelliert.

Fig. 5: An Example for a regionalized temperature scenario during the winter season (Matulla et al. 2004). The upper panel (a) depicts the temperatures derived from observations (1961-1990), the lower panel (b) for 2041-2050 derived from the 'business as usual' scenario as obtained from the ECHAM global scenario. Temperature increases are modelled for Austria.

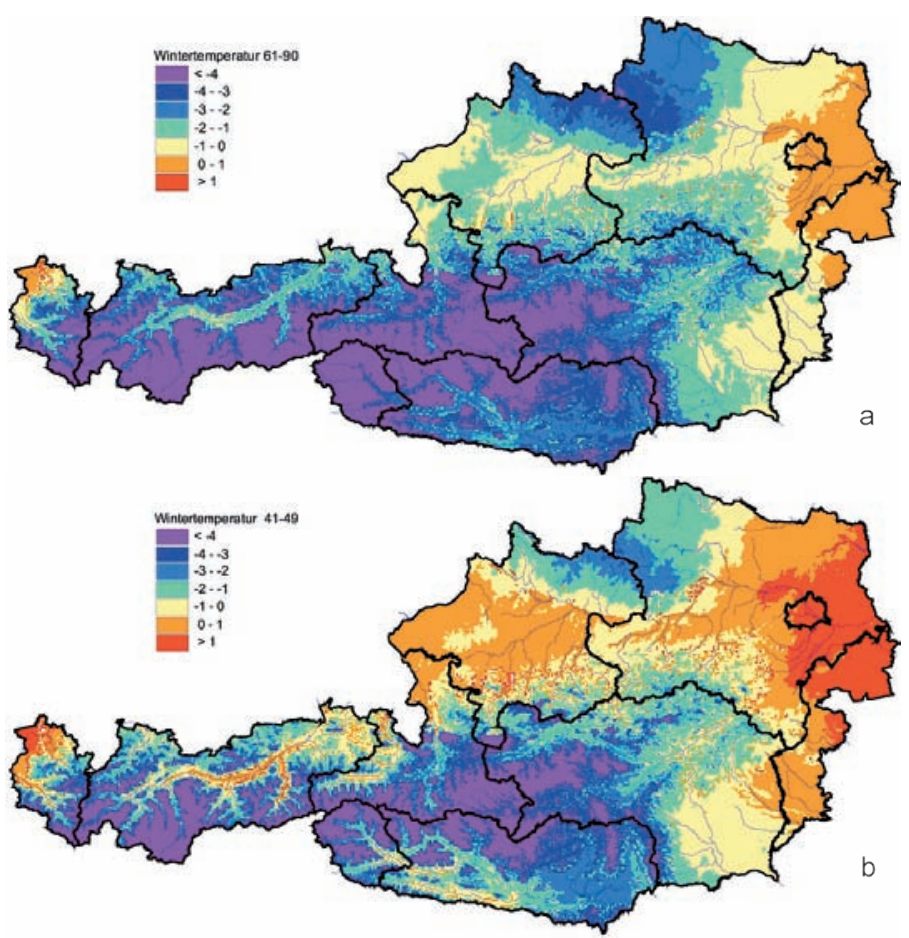

Die Temperaturzunahme verursacht weiterhin eine Verlängerung der Vegetationsperiode (siehe weiter hinten im Text) und damit eine Zunahme der Transpiration, welche zu trockeneren Bedingungen führt. Temperaturzunahme bedeutet auch ein Abschmelzen der Gletscher und daher mittelfristig eine Zunahme der Abflussmenge im Hochsommer. Langfristig führen steigende Temperaturen zu einem Rückgang der Gletscherspende und häufigeren Niederwasserständen während hochsommerlicher Schönwetterperioden.

\section{Die nächsten 100 Jahre - womit gerechnet werden darf}

Die Folgen des Klimawandels in den nächsten 100 Jahren sind ausführlich in IPCC 2007 (Chapter 11) wiedergegeben. Für Europa ergibt sich nach dem A1B Szenario ein annualer Temperaturanstieg um mehr als 3 Grad (2080-2099 verglichen mit 1980-1999). Im nördlichen Europa treten die deutlichsten Temperaturzunahmen im Winter auf, während im Mittelmeerraum die Sommer die stärksten Zuwächse (in beiden Fällen mehr als $4 \mathrm{Grad}$ ) verzeichnen. Das übertrifft den Temperaturanstieg der letzten 150 Jahren in Mitteleuropa, der bei etwa 2 Grad lag. 
Die Variabilität zwischen den Szenarien kann bis zu +2 Grad betragen (B1: +2 Grad, A1: bis +6 Grad). Die Temperaturverteilungen verschieben sich hin zu größeren Werten, wobei die niedrigen Perzentile (Schranken, unterhalb denen nur wenige Werte liegen) etwas weniger Veränderung erfahren als die höheren. Heiße Tage nehmen zu, kalte ab. Es ist mit einer Zunahme an Hitzewellen in Frequenz und Dauer zu rechnen und mit einer Abnahme von Kälteeinbrüchen. Die Tag zu Tag wie auch die interannuelle Variabilität nimmt im Winter in weiten Teilen Europas ab und im Sommer in Mitteleuropa zu.

Wenig Veränderung kann im Sturmklima erwartet werden. Projektionen, die auf den deutschen und englischen globalen Klimamodellen beruhen, deuten auf gleichbleibende Verhältnisse in Mitteleuropa hin. Dieses Verhalten deckt sich mit Analysen für die Periode von den 1880ern bis in die jüngste Vergangenheit (Matulla et al. 2007). Für andere Teile Europas zeigen die Projektionen z.T. gegensätzliche Entwicklungen. Generell werden moderate Veränderungen im mittleren bodennahen Wind simuliert.

Bis 2050 ist ein Rückgang der aktiven Alpengletscherfläche auf 40 bis 50\% der Fläche von 1980 zu erwarten und bis 2100 ein Rückgang auf 15-20\%. Für Österreich ist eine noch stärkere Abnahme wahrscheinlich. Auch die feste Komponente des Niederschlages am Gesamtniederschlag wird anteilmäßig weiter abnehmen.

Niederschlagsprojektionen sind generell mit einer gegenüber den Temperaturprojektionen deutlich erhöhten Unsicherheit verbunden. Die Ergebnisse der Modellsimulationen sind daher weniger gut abgesichert. Nordwärts der Alpen bis nach Fennoskandien sind Zunahmen in den Niederschlagssummen zu erwarten, die im Winter im Mittel bis zu 15\% stärker ausgeprägt sind als im Sommer (wo wenig Veränderung erwartet werden kann). Im Mittelmeerraum hingegen zeigen die Projektionen für alle Saisonen Abnahmen, die im Sommer im Mittel 24\% betragen. Auch diese Veränderungen übertreffen die gemessenen regionalen Langfristtrends der letzten 200 Jahre, die 10\% pro Jh. nicht überschritten haben.

Das deutsche Klimamodell deutet eine Verlagerung des Subtropenhochs nach Nordost an und damit ein häufigeres Auftreten der sommerlichen stabilen Schönwetterlagen in unseren Breiten. Dieses Phänomen findet sich nicht beim englischen Klimamodell. 


\section{Der Impact - ein paar Bemerkungen}

Regionalskalige Klimaänderungsszenarien (Projektionen) dienen auch dazu, die möglichen Auswirkungen des Klimawandels auf Ökosysteme abzuschätzen. Lexer et al. 2002 etwa untersucht waldökologische Reaktionen auf den Klimawandel. Damit werden potentielle Veränderungen in der Zusammensetzung des Baumbestandes des österreichischen Waldes beschrieben (siehe Abbildung 6). Solche Studien können eine Grundlage für zukunftsorientierte forstwirtschaftliche Planung sein.

\section{$\square$ pioneer species \\ $\square$ other species \\ $\square$ Qu. pubescens \\ $\square$ Qu. cerris \\ $\square$ Qu. petraea \\ $\square$ Qu. robur \\ -Larix decidua \\ a Pinus sylvestris \\ $\square$ Carpinus betulus \\ acer pseud. \\ $\square$ Fagus sylvatica \\ - Abies alba \\ $\checkmark$ Picea abies}

Abb. 6: Simulierter, naturnaber' Fichten/Tannen/Buchenwald in der montanen Höhenstufe (Hauptwuchsgebiet 4) unter aktuellem Klima und drei Klimaänderungsszenarien, die in einem mit dem deutschen globalem Modell erzengten , business as usual' Szenario empirisch downgescaled sind (Lexer et al. 2001).

Fig. 6: Simulation of a spruce/ fur/beech forest at the montane belt (primary distribution area). The different panels stand for a continuation of the current climate and three climate change scenarios downscaled empirically from the German global model using a 'business as usual scenario' (Lexer et al. 2001).

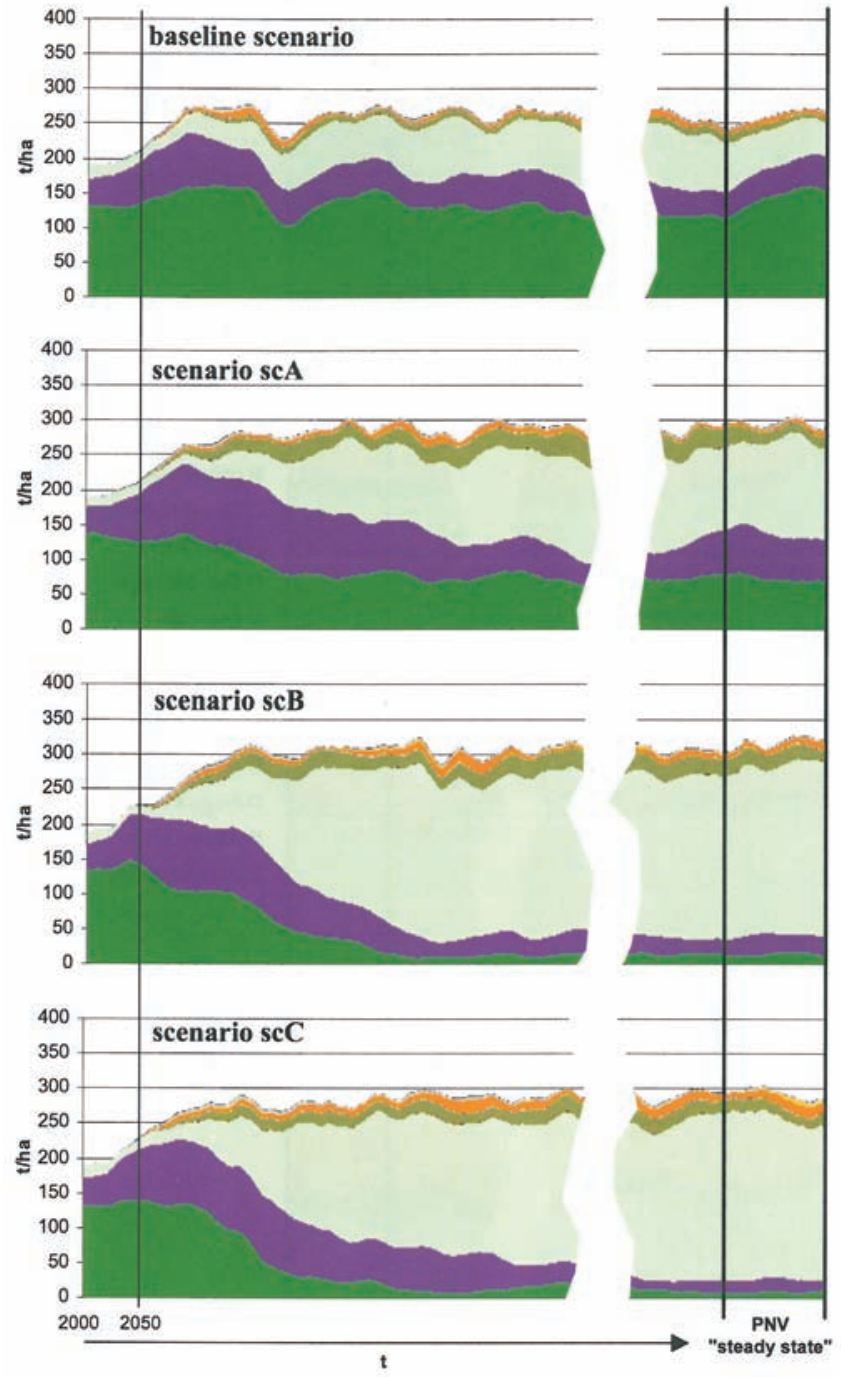


Regionale Projektionen werden auch zur Abschätzung der Auswirkungen auf die Fischvergesellschaftung genutzt. In Österreich ist das erstmals am Beispiel des inneralpinen Flusses Mur umgesetzt worden (Matulla et al. 2007). Die hierbei erzielten Ergebnisse deuten darauf hin, dass die simulierten Veränderungen in Durchfluss und Wassertemperatur bis 2050 eine Verschiebung der Fisch-Spezies um rund $20 \mathrm{~km}$ der Quellenregion entgegen auslösen können. Das erhöht den Druck auf kälteliebende Salmoniden (Bachforelle, Äsche, Huchen) durch das Nachrücken von wärmeliebenden Cypriniden (Karpfen, Barbe, Nase, Aitel). Die modellierten Veränderungen deuten auf nachteilige Effekte hinsichtlich der in der Sportfischerei beliebten und ökonomisch wertvollen Salmonid-Gewässer.

Auswirkungen von Klimaveränderungen auf die Phänologie (die Lehre vom Jahresablauf periodisch wiederkehrender Wachstums- und Entwicklungserscheinungen der Tier- und Pflanzenwelt) wurden ebenso untersucht (z.B. Scheifinger et al. 2002; Matulla et al. 2003). Eine kürzlich erschienene Untersuchung weist auf die Verschiebung von Eintrittsphasen phänologischer Pflanzenphasen in einem sich ändernden Klima hin (Scheifinger et al. 2007). Abbildung 7 zeigt die Ergebnisse für die Phase ,Flieder - erste Blüte'. Diese Resultate beruhen auf Ensembles von Klimaläufen mit ECHAM, angetrieben mit den A1B und B1 Szenarien. Betrachtet man Frühlings- und Herbstphasen, so kann man insgesamt auf eine Verlängerung der Vegetationsperiode schließen. Diese ist vor allem auf ein früheres Auftreten der Frühlingsphasen zurückzuführen. Demgegenüber ist beim Beginn der Herbst-

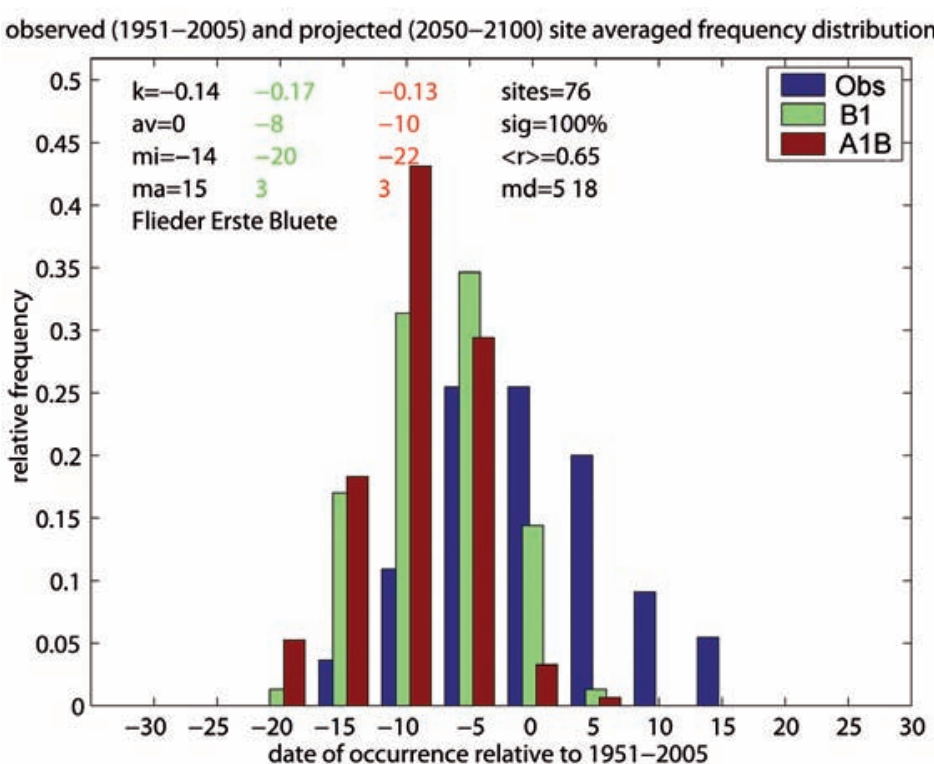

Abb. 7: Verteilung der Einrittstermine der ersten Blüte des Flieders in Österreich. Die Szenarien (A1B und B1, siebe Legende) sind aus Ensembles an Klimaläufen des Klimamodells ECHAM5 abgeleitet. Die Vorverschiebung der Eintrittstermine ist beim A1B EmissionsSzenario stärker ausgeprägt als beim B1 Szenario (Scheifinger et al. 2007).

Fig. 7: Lilac flowering in Austria. Scenarios A1B and B1 are obtained from ECHAM5. The earlier start of blossoming in the $\mathrm{A} 1 \mathrm{~B}$ scenario is more pronounced than in the B1 scenario (Scheifinger et al. 2007). 
phasen kein eindeutiger Trend festzustellen. Die geringere Klarheit bei den Herbstphasen kann mit den Problemen bei ihrer Modellierung im Zusammenhang stehen. Die eingesetzten empirischen Modelle zeigen eine gute Performance am Beginn des Jahreszyklus, die zu späteren Terminen hin abnimmt.

\section{Abschließende Bemerkungen}

Die Entwicklungen der letzten Jahrzehnte auf dem Feld der Klimaforschung im Allgemeinen und bei der Klimamodellierung im Besonderen waren und sind von großer Dynamik geprägt. Eine wachsende Menge an Klimamodellen und Klimamodellversionen begleitet die immer realitätsnäher werdende Beschreibung der physikalischen Prozesse im Klimasystem. Dies resultiert zusammen mit den oben beschriebenen verschiedenen Emissionsszenarien in einer Vielzahl an Simulationen.

Das ist eine zu begrüßende Ausgangslage, denn Impactforschung muss sich heutzutage durchaus nicht stets am aktuellsten Modelllauf orientieren. Bisher galten Ergebnisse von Impactforschung als zum Teil überholt, wenn von der Klimaforschung neue Szenarien zur Verfügung gestellt wurden. Mit der Verfügbarkeitswerdung einer immer größeren Menge an Klimaszenarien sinkt diese Abhängigkeit.

Soll eine Bewertung möglicher Auswirkungen von Klimaänderungen auf Ökosysteme vorgenommen werden, ist es zweckdienlich, diese auf die Grundlage einiger unterschiedlicher Klimaszenarien zu stellen. Ein einzelner Klimasimulationslauf (unabhängig davon, wie aktuell er ist) liefert eine einzelne Schätzung. Eine Menge an Szenarien gestattet eine Beschreibung der Auswirkungen auf Ökosysteme unter unterschiedlichen (künftigen) Klimabedingungen.

Ergebnisse der Impactforschung sind daher nicht mehr automatisch mit dem Release einer neuen Klimamodell-Generation überholt. Vor allem dann nicht, wenn sie auf einer für die zu untersuchende Fragestellung dienlichen Menge an Szenarien aufsetzen.

Die oben dargestellten Auswirkungen einer Klimaänderung auf den österreichischen Wald oder die Fischvergesellschaftung der Mur beruhen noch auf Einzelschätzungen, die Analyse der Auswirkungen auf die Phänophasen bereits auf Ensembleläufen des ECHAM Modells unter zwei verschiedenen Emissionsszenarien. Die Entwicklung der Klima- und Impactforschung führt in diesem Sinne zu immer belastbareren Aussagen über das künftige Klima und dessen Auswirkungen auf die Umwelt. 


\section{Klimawandel in Österreich}

\section{Literatur}

IPCC, 2001: Climate Change 2001: The Scientific Basis. Summary for Policymakers and Technical Summary of the Working Group I Report, Cambridge 2001, p. 63.

IPCC, 2007: Climate Change 2007: The Physical Science Basis. Contribution of Working Group I to the Fourth Assessment Report of the IPCC, Cambridge 2007, Chapter 10.

Lexer, M.J., K. Hoenninger, H. Scheifinger, C. Matulla, N. Groll, H. Kromp-Kolb, K. Schaudauer, F. Starlinger and M. Englisch, 2001: The sensitivity of the Austrian forests to scenarios of climatic change. A large-scale risk assessment, Monographien. Umweltbundesamt/Federal Environment Agency-Austria, 132, 132 pp.

Lexer, M.J., K. Hoenninger, H. Scheifinger, C. Matulla, N. Groll, H. Kromp-Kolb, K. Schaudauer, F. Starlinger, and M. Englisch, 2002: The sensitivity of Austrian forests to scenarios of climate change: a large-scale risk assessment based on a modified gap model and forest inventory data, Forest Ecology and Management, 162, 53-72.

Matulla C., W. Schoener, H. Alexandersson, H. von Storch, X.L. Wang 2008: European Storminess: Late 19th Century to Present, Climate Dynamics, 31 (2-3), 125-130.

Matulla, C., S. Schmutz, A. Melcher, T. Gerersdorfer and P. Haas 2007: Climatic Change impact on fish fauna for an Inner-Alpine River based on a transient AOGCM simulation, International Journal of Biometeorology 52, 127-137.

Matulla, C., H. Formayer, P. Haas, and H. Kromp-Kolb, 2004: Possible climate trends in Austria in the first half of the 21st century, OeWAW, 56, 1-9.

Matulla, C., H. Scheifinger, A. Menzel, and E. Koch, 2003: Exploring two methods for statistical downscaling of Central European phenological time series, International Journal of Biometeorology, 48, 56-64.

Scheifinger H., A. Menzel, E. Koch, C. Peter, R. Ahas, 2002: Atmospheric mechanisms governing the spatial and temporal variability of phenological phases in central Europe, International Journal of Climatology 22 (14), 1739-1755.

Scheifinger H., C. Matulla, P. Cate, A. Kahrer, E. Koch, 2007: Climate impact on plant and insect phenology in Austria (http://epub.oeaw.ac.at/3966-9inhalt).

von Storch, H., 1995: Inconsistencies at the interface of climate impact studies and global climate research, Meteorol. Z., N.F. 4, 72-80.

von Storch, H., E. Zorita and U. Cubasch, 1993: Downscaling of global climate change estimates to regional scales: An application to Iberian rainfall in wintertime, J. Climate 6, 1161-1171.

Zorita E. and H. v. Storch, 1999: The analog method as a simple statistical downscaling technique: comparison with more complicated methods, Journal of Climate 12, 2474-2489. 


\title{
Zusammenfassung und Ausblick
}

\author{
Roland Schmidt ${ }^{1)}$, Christoph Matulla ${ }^{2)}$ und Roland Psenner ${ }^{3)}$ \\ 1) Institut für Limnologie, Österreichische Akademie der Wissenschaften, Mondsee, \\ Österreich \\ 2) Zentralanstalt für Meteorologie und Geodynamik (ZAMG), Wien, Österreich \\ 3) Institut für Ökologie der Universität, Innsbruck, Österreich
}

Moränen von Gletschervorstößen, die Baumring-Forschung (Dendrochronologie) und die multidisziplinäre Auswertung von Seesedimenten unter Einbezug von Organismen, die als Indikatoren von Umweltvariablen dienen, weisen für die letzten 20.000 Jahre auf mehrmalige Wechsel von kälteren und wärmeren Perioden hin. Der Temperaturanstieg nach dem Ende der letzten Eiszeit wurde durch mehrere Kälteschwankungen unterbrochen. Von den klassischen alpinen Gletschervorstößen (Stadialen) sind Gschnitz und Egesen Ankerpunkte in einer sich noch im Aufbau befindlichen gletscherkundlichen Chronostratigraphie des ausklingenden Glazials. Diese Stadiale sind jedoch auch ein Beispiel dafür, wie in zunehmenden Maße versucht wird, die durch verschiedene Proxies (=Indikatoren, Klimazeiger) rekonstruierten Klimaschwankungen in einem größeren, zumindest nordhemisphärischen Rahmen zu betrachten. Dabei bieten sich vor allem die Sauerstoffisotopen $\left(\delta{ }^{18} \mathrm{O}\right)$ grönländischer Eiskerne oder Ereignisse in dem als „europäische Klimaküche" betrachteten Nordatlantik an (z. B. die klimaabhängigen Verschiebungen in der nordpolaren Packeiszone und Eisdrift = "ice rafting events"). Die Amplituden dieser Klimaschwankungen nahmen in der späten Eiszeit stufenartig ab und pendelten sich in den letzten ca. 11.500 Jahren (= Holozän) auf eine relativ enge Bandbreite von etwa $\pm 1.5^{\circ} \mathrm{C}$ ein. Die Kälteschwankungen (= Klimaoszillationen) des Holozäns zeigen eine annähernd tausendjährliche Wiederkehr, deren Steuermechanismen wohl extraterrestrischer Natur sind, wie zum Beispiel Veränderungen der Erdlaufbahn um die Sonne. Hinzu treten kurzfristige, zumeist azyklische, Klimafluktuationen, wie zum Beispiel die oft zitierte Kälteschwankung um 8200 vor heute, sowie Extremereignisse, die anderen Steuermechanismen unterliegen dürften. Zwischen den Moränen des ausklingenden Spätglazials (Egesen) und jenen des 1850er Hochstandes sind in den Alpen noch weitere Moränenstaffeln eingeschachtelt, die bisher nicht alle mit den durch andere Indikatoren angezeigten Klimaschwankungen des Holozäns in Übereinstimmung gebracht werden konnten. 
Heute beginnt man sich auch verstärkt jenen Zeiträumen zuzuwenden, in denen die Alpen, ähnlich wie für die nahe Zukunft prognostiziert, nur eine geringe Gletscherbedeckung zeigten. Solche Warmperioden waren das älteste Holozän, die in einzelne Warmphasen ("events") aufgespaltene sogenannte postglaziale Wärmezeit, Teile der Bronzezeit, die Römerzeit und Abschnitte des Früh- und Hochmittelalters. Von diesen Warmperioden wiesen die Römerzeit und das Hochmittelalter ähnliche Temperaturen wie heute auf. Der Vergleich mit der aktuellen Klimaerwärmung hinkt jedoch, da es erwiesen ist, dass der Mensch durch den Ausstoß von Treibhausgasen in die Atmosphäre den Prozess der globalen Klimaerwärmung mit verursacht und beschleunigt. Hinzu kommen neuerdings Indizien, dass sich die Jahreszeiten unterschiedlich entwickeln, sodass der „, saisonalen Klimaforschung“ besondere Bedeutung für das Verständnis der komplexen Klimaabläufe zukommt. In den Warmzeiten verschiebt sich auch die Grenze des dauerhaft gefrorenen Bodens (Permafrost) in den Alpen nach oben, wie dies derzeit mit all ihren Konsequenzen, wie z.B. der Destabilisierung von Berghängen mit folgenden Bergstürzen, sichtbar wird bzw. Ziel aktueller Forschung ist, die den komplexen Wechselbeziehungen zwischen Klima, Böden und Gewässern nachgeht (z.B. die verstärkte Freisetzung von Schwermetallen aus dem tauenden Permafrost). Hinzu kommen klimagesteuerte Verschiebungen in der Biodiversität und verborgene, da unterhalb des Artniveaus ablaufende „mikroevolutive" Aspekte, die jedoch mit Hilfe molekulargenetischer Techniken sichtbar gemacht werden können. Den ausgeprägten Warmzeiten steht die im 16. Jh. beginnende so genannte „Kleine Eiszeit" gegenüber, die im neuzeitlichen Gletscherhochstand um 1850 gipfelte. Wenn auch die Bilanzierung der Gletscher ein aufwändiges Verfahren ist, so zeigen sich dennoch im Vergleich zu diesem Hochstand die erheblichen Massenverluste der Alpengletscher während der letzten Dekaden. Sie sind ein sichtbarer Ausdruck der rezenten Klimaerwärmung. Diesem Gletscherverlust steht die Neubildung von Seen gegenüber, für deren Wiederbesiedelung die Vergangenheit wichtige Referenzdaten zu liefern vermag. Wesentlich schwieriger fassund rekonstruierbar, da deutlich variabler als die Temperatur, ist der Niederschlag. Ähnlich wie für die Temperatur sind jedoch auch hier Langzeittrends, Oszillationen, saisonale und räumliche Aspekte wie zum Beispiel die klimatische Heterogenität zwischen Nord und Süd, West und Ost, sowie kurzfristige Fluktuationen und Extremereignisse zu berücksichtigen. Im Langzeittrend über Jahrtausende ist allgemein eine Entwicklung von den kontinental geprägten Verhältnissen des Spätglazials zu humideren des Holozäns feststellbar. Diese Entwicklung scheint Hand in Hand mit Veränderungen der Küstenlinien im Zuge des globalen holozänen natürlichen 
Meeresspiegelanstiegs zu gehen. So entwickelten sich im Laufe des Holozäns die heutigen Klimabezirke der Alpen als eine von West nach Ost laufende Barriere im Einflussbereich zwischen dem ozeanisch geprägten Klima des Atlantiks, dem adriatisch-mediterranen, sowie kontinentalen Klima. Die Einflussgrößen, wie etwa die hauptsächlich das Winterklima in den Alpen beeinflussende Nordatlantische Oszillation (NAO) und Zyklone des nördlichen Mittelmeers (z.B. Genua Tief), scheinen jedoch nicht konstant gewesen zu sein, wie das Beispiel eines seit dem Mittelalter wieder kontinentaler gewordenen inneralpinen Raums vermuten lässt und durch Klimadaten der letzten 130 Jahre erhärtet wird. Bei der Suche nach weiteren Proxies für die Rekonstruktion des Niederschlags in der Vergangenheit könnte sich in den Ostalpen auch die niederschlagsabhängige Baumringbildung bei der Schwarzföhre als hilfreich erweisen. Der Einfluss der Meere als Steuermechanismen für Veränderungen großräumiger Zirkulationsströmungen und deren Einfluss auf die Alpen, wie etwa die Interaktionen zwischen Atlantik (El Niño/La Niña) und Mittelmeer, ist verstärkt Ziel aktueller Forschung.

Dieser Band zeigt jedoch auch, wie sehr sich die Rolle des Menschen in den vergangenen Jahrtausenden verändert hat: von der weitgehenden Klimaabhängigkeit in prähistorischen Zeiten über eine bedarfsorientierte Sozioökonomie am Beispiel Hallstatt bis hin zur modernen Zeit, wo der Mensch selbst aktiv in das Klimageschehen eingreift. Der vorliegende Band vermittelt einen Einblick, wie vielschichtig das Klima im Alpenraum ist, wenn wir von den homogenisierten instrumentellen Daten der letzten Jahrhunderte zur Klimadynamik größerer Zeiträume übergehen und welche möglichen natürlichen Einflussgrößen es zu berücksichtigen gilt, wenn realistische Modelle und Zukunftsszenarien für die Alpen im Klimawandel entwickelt werden sollen. Der Band zeigt auf, dass wir, wenn wir realistische Szenarien für das Klima des 21. Jahrhunderts entwickeln wollen, noch sehr viel über das Klima der Vergangenheit, das heißt des gesamten Holozäns, zu lernen haben. Dieses Ziel werden wir nur mit innovativen und interdisziplinären Forschungsansätzen und mit Hilfe internationaler Forschungsnetzwerke erreichen. Da die Alpen nicht nur das Wasserschloss Europas, sondern auch ein eminenter Klimafaktor für den gesamten Kontinent sind, werden sie auch in Zukunft für die Klimaforschung eine zentrale Rolle spielen. 


\section{Bisherige Publikationen in der Reihe alpine space - man \& environment}

vol. 1

Die Alpen im Jahr 2020

Roland Psenner, Reinhard Lackner (Hrsg.)

2006, brosch., 121 Seiten

ISBN: 978-3-902571-01-4

vol. 2

Politische, kulturelle und wissenschaftliche Perspektiven der nachhaltigen Raumentwicklung in den Alpen

Axel Borsdorf, Sigrun Lange (Hrsg.)

2006, brosch., 135 Seiten

ISBN: 978-3-902571-02-1

vol. 3

The Water Balance of the Alps

What do we need to protect the water resources of the Alps?

Roland Psenner, Reinhard Lackner (Hrsg.)

2007, brosch., 86 Seiten

ISBN: 978-3-902571-33-5

vol. 4

Ist es der Sindtfluss?

Kulturelle Strategien \& Reflexionen zur Prävention und

Bewältigung von Naturgefahren

Roland Psenner, Reinhard Lackner, Maria Walcher (Hrsg.)

2008, brosch., 128 Seiten

ISBN: 978-3-902571-32-8

vol. 5

Über Almen

Zwischen Agrikultur \& Trashkultur

Christoph Kirchengast

2008, brosch., 148 Seiten

ISBN: 978-3-902571-46-5

\section{Sonderband}

Die Alpen

Einblicke in die Natur

Rudolf Hofer (Hrsg.)

2009, brosch., 160 Seiten

ISBN: 978-3-902719-02-7 
